$$
\text { DOE/ER/53093 - - } 1
$$

THE ALFVÉN WAVE

\title{
MASTER by
}

Akira Hasegawa

Niember of Technical Staff

Bell Laboratories

Murray Hill, New Jersey 07974

$$
\text { U.S.A. }
$$

Adjunct Professor Columbia University New York, New York

$$
\text { U.S.A. }
$$

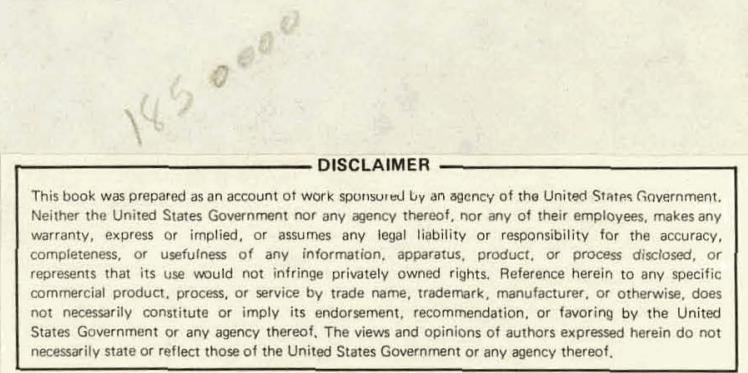

Chanchal Uberoi

Associate Professor Department of Applied Mathematics Indian Institute of Science

Bangalore, India 


\section{DISCLAIMER}

This report was prepared as an account of work sponsored by an agency of the United States Government. Neither the United States Government nor any agency Thereof, nor any of their employees, makes any warranty, express or implied, or assumes any legal liability or responsibility for the accuracy, completeness, or usefulness of any information, apparatus, product, or process disclosed, or represents that its use would not infringe privately owned rights. Reference herein to any specific commercial product, process, or service by trade name, trademark, manufacturer, or otherwise does not necessarily constitute or imply its endorsement, recommendation, or favoring by the United States Government or any agency thereof. The views and opinions of authors expressed herein do not necessarily state or reflect those of the United States Government or any agency thereof. 


\section{DISCLAIMER}

Portions of this document may be illegible in electronic image products. Images are produced from the best available original document. 
To Professor Hannes Alfvén, the discoverer of the wave which has stimulated so much important research in plasma physics. 


\section{PREFACE}

of the many types of waves that exist in plasmas, the Alfvern wave is probably the best studied. However, some of its unique properties such as the absence of a discrete eigenmode in a nonuniform plasma and the effect of a finite ion gyroradius are not widely known. While the importance of the Alfvén wave in astrophysical and space plasmas was realized soon after its discovery in 1942, plasma physicists paid relatively less attention to it until recently due to difficulties in exciting it in the laboratory. However, the recent progress in thermonuclear fusion research has increased the possible physical size and magnitude of plasma $B$ (the ratio of the plasma to magnetic field pressure) so that the Alfvén mode is now accessible in laboratory plasmas. Consequently a large part of this book explores areas of relevance of Alfvên waves to fusion. However, the monograph is prepared so that it can also serve as a good review for. scientists in the areas of astrophysics, space physics and solid state physics. Recent references in these areas are listed at the end of Chapter I.

The monograph contains seven chapters. 'Chapters I and II introduce the Alfvên wave and describe its linear properties in a homogeneous medium. Chapters III and IV cover the effects of inhomogeneities on these linear properties. 
Particular emphasis is placed on the appearance of a continuum spectrum and the associated absorption of the Alfuén wave which arise due to the inhomogeneity. The explanation of the physical origin of absorption is given using kinetic theory. Chapter $V$ is devoted to the associated plasma instabilities.

In recent publications considerable interest has been shown in the study of nonlinear phenomena associated with the Alfvén wave. As it is not possible to describe all this work we restricted ourselves to the study of nonlinear processes in laboratory scale plasmas. Nonlinear effects discussed in Chapter VI include quasilinear diffusion, decay, a solitary wave and a modulational instability. To compensate for our omissions, we have included a large number of references on recent work at the end of the chapter.

The Alfuén wave is a potential candidate for R.F. heating of a fusion plasma because low cost power suiries. in the appropriate frequency range are readily available and its absorption rate is high. The principles of Alfién wave'. heating, a design example and present-day experimental results are described in chapter VII.

The idea for this monograph originated when the authors met at Bangalore, India, in December of 197.6. It has been difficult to co-author a book while sitting on opposite 
sides of the earth, but thanks to our very efficient typists, MS. Hildegard Franks (U.S.A.) and Mr. K. S. Rajagopal (India), it has gone rather smoothly.

After the manuscript was finished, one of the authors (A.H.) used it in a plasma seminar course at Columbia graduate school, to attempt to teach plasma physics using the Alfvern wave as an example. The richness of phenomena related with the Alfven wave which involves the MHD theory, the kinetic theory, plasma instabilities and nonlinear effects is found to be very useful in showing how the plasma physics theory can be applied to a real problem.

We are both grateful to our spouses, Miyoko Hasegawa and Professor S. N. Balasubrahmanyam for their encouragement and endurance. On of the authors (A.H.) is particularly thankful to his favorite music colleague, Sachiko Nishida, who has continuously helped to calm his impatient spirit. The other (C.U.) is thankful that her children. Raju and sunu "understood" when their mother had to go to the "office".

\section{Akira Hasegawa}




\section{CONTENTS}

I. ALFVÉN WAVE - 'IDEAL'

1.1. Introduction

1.2 Dispersion Relation

1.3 Properties.

1.4 Reflection and Transmission

1.5 Finite Conductivity

1.6 Compressibility Effects

1.7 Coriolis Force Effects

1.8 Cylindrical Geometry: Torsional

Alfvén Wave

1.9 Experimental studies

References

II. ALFVEN WAVES IN PLASMAS

2.1 Introduction

2.2 Dispersion Relation

2.3 Compressional Alfvén Wave

2.4 Hall-Current Effects: Finite Frequency

Alfvén Wave

2.5 Ion-Neutral Collision Effects

2.6 Finite Larmour Radius Effects: Kinetic

A1.fvén Wave

2.7 Zlfvến Waves in Cylindrical Plasmas

2.8 Experimental Study of Alfvén Wave Properties

2.9 Concluding Remarks

References 
III. SPECTRAL ANALYSIS OF IDEAL ALFVÉN WAVE IN INHOMOGENEOUS MAGNETIC FIELDS

3.1 Introduction

3.2 The Wave Equation

3.3 Solution of the Wave Equation

3.4 Concluding Remarks References

IV. RESONANT ABSORPTION AND MODE CONVERSION OF ALFVEN SURFACE WAVES IN NON-UNIFORM PLASMAS

4.1 Introduction

4.2 Alfvén Surface Wave

4.3. Resonant Absorption of Surface Wave

4.4 Resonant Mode Conversion

References

V. PLASMA INSTABILITIES ASSOCIATED WITH THE ALFVÉN WAVE

5.1 Introduction

5.2 Fire Hose Instability

5.3 Two stream Instability

5.4 Kelvin-Helmholtz Instabiluty

5.5 Drift Alfvén Wave Instability

References 
VI. NONLINEAR PROCESSES

6.1 Introduction

6.2 Quasi-Linear Diffusion

6.3 Parametric Decay

6.4 Solitary Wave of the Kinetic Alfvén Wave

6.5 Modulational Instability and Envelope

Soliton

References

VII. PLASMA HEATING BY ALFVÉN WAVE

7.1 Introduction

7.2 Resonant Absorption and Energy

Deposition Rate

7.3 Heating Rate

7.4 Some Design Examples

7.5 Experimental Results

References

Appendix Equations Used in the Book

List of Symbols

subject Index 


\section{ALFVÉN WAVE - 'IDEAL'}

\subsection{Introduction}

The well known basic principle of magnetohydromagnetics is that when a conducting fluid moves in the presence of a magnetic field, the motion of the fluid gives rise to electromagnetic, force which produces electric currents. These electric currents modify the initial magnetic field and in the presence of the magnetic field the mechanical force due to the electric currents modifies the motion of the fluid. Thus there is an interaction between the magnetic field and the motion of the fluid. The mechanical effects of a magnetic field are equivalent to a hydrostatic pressure $B^{2} / 2 \mu_{0}$, combined with a tension $\mathrm{B}^{2} / \mu_{0}$ along the lines of force. In an incompressible fluid the hydrostatic pressure can be balanced by the pressure of the fluid so that only the tension $\mathrm{B}^{2} / 2 \mu_{0}$ remains effective. Analogy with the theory of stretched strings suggests that this tension may lead to the possibility of transverse waves along the lines of force, with a velocity $v_{A}$ given by

$$
v_{A}^{2}=B^{2}\left(\mu_{0} \rho\right)^{-1}
$$

where $\rho$ is the mass density of the fluid. 
The possibility of such waves was first established by Alfvên in 1942. The name given by Alfvên was 'electromagnetic-hydrodynamic.' waves.

1.2 Dispersion Relation

To study small amplitude wave propagation in an ideal incompressible fluid we linearize the ideal MHD equations (Appendix) taking $\rho$ to be constant and $\sigma \rightarrow \infty$, an infinite conductivity assumption. The fluid is initially stationary and equilibrium magnetic field is $\underset{\sim}{\mathrm{B}_{0}}=\left(0,0, \mathrm{~B}_{0}\right)$. We consider a linearly polarized wave with the wave magnetic and velocity fields are in the $y$-direction;

$$
B_{x}=B_{z}=v_{x}=v_{z}=0
$$

We take the direction of wave propagation in $x-z$ plane. This is equivalent to take an incompressible perturbation, $\nabla \cdot v=0$. Using $(\underset{\sim}{v} \cdot \underset{\sim}{\nabla}) \underset{\sim}{v}=v_{y} \frac{\partial v_{y}}{\partial y}=0$, the equation of motion of fluid becomes,

$$
\rho \frac{d v_{y}}{d L}=\rho \frac{\partial v_{y}}{\partial t}=-J_{x^{B}}{ }^{\prime}
$$

where the $x$ component of the current density $J_{x}$ relates to By through the Amperé's law,

$$
\mu_{0} J_{x}=-\frac{\partial B}{\partial z}
$$

$B_{y}$ and $v_{y}$ are related through the electric field by ohm's law

$$
E_{x}+v_{y_{0}^{B}}=0
$$


and the Maxwell equation

$$
\frac{\partial B_{y}}{\partial t}=-\frac{\partial E_{x}}{\partial z}
$$

If we substitute Eqs. (1.2), (1.4) and (1.5), into (1.3), we have,

$$
\frac{\partial^{2} B_{Y}}{\partial t^{2}}=\frac{B_{0}^{2}}{\mu_{0}^{\rho}} \frac{\partial^{2} B_{Y}}{\partial z^{2}}
$$

and

$$
v_{Y}=-\frac{1}{\sqrt{\mu_{0}^{\rho}}} B_{Y}=-v_{A} \frac{B_{y}}{B_{0}} .
$$

Equation (1.2) represents a wave propagating along z-direction with velocity $v_{A}$ given by $\quad \pm B_{0}\left(\mu_{0} \rho\right)^{-1 / 2}$. The sinusoidal solution of Eq. (1.6) is

$$
{ }^{B}{ }_{Y}=A \sin \omega\left(z / v_{A}-t\right)
$$

and so

$$
v_{y}=-\dot{A} \frac{1}{\sqrt{\mu_{0}^{\rho}}} \sin \omega\left(z / v_{A}-t\right)
$$

The lines of force, now lie in the $y^{-z}$ plane and their shape is given by the equation

$$
\frac{d y}{d z}=\frac{B_{y}}{B_{0}}
$$

Solving this we find that $\frac{d y}{d t}=v_{y}$, i.e., the velocity of the lines of force is the same as the fluid velocity. This leads 
to the well known concept of "frozen-in" fields. The magnetic lines of force are "frozen in" the conducting fluid and are thereby constrained to move with the fluid. It is this concept which gives rise to the analogy of Alfvern waves to those waves along an elastic string. The magnetic lines of force have a lateral pressure and a tension equal to $\mathrm{B}^{2} / \mu_{0}$. As the magnetic lines are frozen in the fluid the density can be taken as $\rho$, the fluid density. Hence, the wave velocity along the magnetic Iines of force is given as $\mathrm{B}_{0}\left(\mu_{0} \rho\right)^{-1 / 2}$. We also note that the wave equation $(1.6)$ is exact in that we did not have to linearize to obtain it $[(v \cdot \nabla) v$ term in Eq. (1.2) is identically zero]. In this respect, the Alfvén wave is considered as an exact solution. 


\subsection{Properties}

The most striking property of the Alfven wave is that it.propagates in one direction only, i.e., along the magnetic lines of force which pass through it and therefore suffers no geometric attenuation with distance, i.e., it does not spread out three-dimensionally around a source of disturbance which leads to 'spherical attenuation'. There are cases of astrophysical importance where densities are so low that attenuation due to collisional effects become negligible over relevant length scales.

The Alfvên wave is transverse in nature as the particle velocities are perpendicular to the direction of wave propagation.

It is a low frequency wave, as we have already set an assumption of neglection of the displacement current. The discovery of this wave in an incompressible medium became very important and highly interesting phenomena as the energy could be transmitted without large scale exchanges of the fluid elements.

Alfvén velocities are seen to be several orders of magnitude smaller than velocity of light, cf. the Table I. Because of this velocity the wave can be easily controlled. This fact is used in the coupling of Alfven wave to other waves and as seen in the excitation of sound waves in solid. 


\section{TABLE I}

Media

B

$\mathrm{v}_{\mathrm{A}}$

Solar Corona

$10^{-2}$ Gauss

$10^{-16} \mathrm{gm} / \mathrm{cm}^{3}$
$3 \times 10^{5} \mathrm{~cm} / \mathrm{sec}$

Galactic Arms

$10^{-5}$ Gauss

$10^{-24} \mathrm{gm} / \mathrm{cm}^{3}$ $3 \times 10^{6} \mathrm{~cm} / \mathrm{sec}$

Hyarogen Gas $10^{4}$ Gauss

$10^{-8} \mathrm{gm} / \mathrm{cm}^{3}$ $3 \times 10^{7} \mathrm{~cm} / \mathrm{sec}$ (at 100 micro pressure

Bismuth $10^{3}$ Gauss $10^{-10} \mathrm{gm} / \mathrm{cm}^{3}$ $3 \times 10^{7} \mathrm{~cm} / \mathrm{sec}$ 
In an Alfvén wave the energy is transmitted partly as magnetic and partly as kinetic. The electric energy is small and is insignificant for the case of an Alfvén wave. The kinetic energy density,

$$
\begin{gathered}
\frac{1}{2} \rho v^{2}=\frac{1}{2 \mu_{0}} A^{2} \sin ^{2} \omega\left(z / v_{A}-t\right)=B^{2} / 2 \mu_{0}, \\
(=\text { the magnetic energy density. })
\end{gathered}
$$

Therefore, for a magnetohydrodynamic. Alfvén wave the kinetic energy density is equal to the magnetic energy density. This equi-partition of energy, is of course, a consequence of the "frozen-in" Iines of force and this will, therefore, hold only for medium of infinite conductivity.

However, as will be seen in Sec. 2.6, the Alfvén wave with perpendicular wavelength comparable to the ion gyroradius carries electric energy which is comparable to the magnetic field energy.

\subsection{Reflection and Transmission}

Here we consider reflection and transmission of the Alfvén wave. We take:

$$
\begin{aligned}
& B_{Y}=A \sin \omega\left(z / v_{A I}-t\right), z<0 \text {, incoming wave, } \\
& B_{Y}^{\prime}=A^{\prime} \sin \omega\left(z / v_{A 2}-t\right), z>0 \text {, transmitted wave, } \\
& B_{Y}^{\prime \prime}=A " \sin \omega\left(-z / v_{A l}-t\right), z<0 \text {, reflected wave, } \\
& E_{x}=A v_{A l} \sin \omega\left(z / \ddot{v}_{A l}-t\right) ; z<0,
\end{aligned}
$$




$$
\begin{aligned}
& E_{X}^{\prime}=A^{\prime} v_{A 2} \sin \omega\left(z / v_{A 2}-t\right), z>0, \\
& E_{X}^{\prime \prime}=-A^{\prime \prime} v_{A 1} \sin \omega\left(-z / v_{A 1}-t\right), z<0 .
\end{aligned}
$$

Boundary conditions are:

$$
\begin{aligned}
& B_{y}+B_{y}^{\prime \prime}=B_{y}^{\prime} \\
& E_{x}+E_{x}^{\prime \prime}=E_{x}^{\prime}
\end{aligned}
$$

which gives

$$
A^{\prime}=\frac{2 v_{A 1}}{v_{A 1}+v_{A 2}} A, \quad A^{\prime \prime}=\frac{v_{A 1}-v_{A 2}}{v_{A 1}+v_{A 2}} A \text {. }
$$

Rigid boundary (conducting, $\rho_{2}=\infty$ ) : $A=A$ ", no phase shift in the induced magnetic field. From the relation between $v_{y}$ and $B_{y}$ it can be easily shown that there is $180^{\circ}$ phase shift in the velocity field. Free surface: $\left(\rho_{2}=0\right): A=-A "$, hence there is $180^{\circ}$ phase shift in the magnetic field and velocity field is reflected with same phase. 
At a nonconducting boundary the current density $\mathrm{J}_{\mathbf{x}}$ associated with the wave must be zero, which gives $\mathrm{A}=-\mathrm{A}^{\prime}$. Hence, phase shift in the magnetic and velocity field is same as in the case of the free surface.

A general treatment of the reflection and refraction of Alfvén waves, when the magnetic field is inclined at an angle of the plane of separation $z=0$, has been given by Ferraro(1954). It was shown that the general laws of reflection and refraction no longer remain simple as in the case when the magnetic field is normal to the surface of separation, since they depend on the orientation of the field with respect to the surface of separation of the two liquids.

\subsection{Finite Conductivity}

The 'infinite conductivity' is an ideal situation. What are the conditions for which Alfvén wave can still exist if the conductivity is large but finite? This can be studied by introducing effect of finite conductivity in Ohm's law;

$$
E_{x}+v_{y_{0}} B_{0}=\frac{1}{\sigma} J_{x}
$$


The dispersion relation is

$$
\omega^{2}-\left(v_{A}^{2}+\frac{i \omega}{\mu_{0} \sigma}\right) k^{2}=0 \text {, }
$$

or

$$
k= \pm \frac{\omega}{v_{A}}\left(1+\frac{i \omega}{\mu_{0} \sigma v_{A}^{2}}\right)^{1 / 2},
$$

where $\sigma$ is the conductivity.

For small damping

$$
k \simeq \pm\left(\frac{\omega}{v_{A}}-\frac{i \omega^{2}}{2 \mu_{0} \sigma v_{A}^{3}}\right) .
$$

The damping distance is

$$
\mathrm{z}_{0}=\frac{2 \mu_{0} \sigma \mathrm{v}_{\mathrm{A}}^{3}}{\omega^{2}}
$$

For an Alfvén wave with wavelength $\lambda$ to exist,

$$
z_{0} \gg \lambda, \quad \text { i.e., } \quad L \equiv \frac{B_{0} \lambda \sigma \mu_{0}^{\frac{1}{2}}}{\rho^{\frac{1}{2}}} \gg 1 \text {. }
$$

$\mathrm{L}$ is known as the Lundquest number (Lundquest, 1949a,b).

\subsection{Compressibility Effects}

When compressibility is taken into account the dispersion relation assumes the form

$$
\left[\frac{\omega^{4}}{k^{4}}-\frac{\omega^{2}}{k^{2}}\left(c_{S}^{2}+v_{A}^{2}\right)+c_{S}^{2} v_{A}^{2} \cos ^{2} \theta\right]\left(\frac{\omega^{2}}{k^{2}}-v_{A}^{2} \cos ^{2} \theta\right)=0,
$$


where $c_{S}=\sqrt{\frac{\gamma_{0}}{\rho_{0}}}$ is the sound speed and $\theta$ is the angle between the equilibrium magnetic field direction and the wave propagation direction.

There are three modes of propagation

$$
\frac{\omega^{2}}{k^{2}}=\frac{1}{2}\left(c_{S}^{2}+v_{A}^{2}\right)^{\prime} \pm \frac{1}{2}\left[\left(c_{S}^{2}+v_{A}^{2}\right)^{2}-4 c_{S}^{2} v_{A}^{2} \cos ^{2} \theta\right]^{\frac{3}{2}}
$$

and

$$
\frac{\omega^{2}}{k^{2}}=v_{A}^{2} \cos ^{2} \theta
$$

They are termed as fast, slow (magnetosonic waves) and intermediate modes (Alfvén wave) respectively.

$$
\text { When } \theta=\pi / 2 \text {, the only mode that persists is the }
$$

Fast mode which has about the same velocity as the Alfvern speed in the case of magnetic pressure being large compared to the material pressure and represents the compressional Alfven mode. The mode arises due to compression of the magnetic lines of force.

$$
\begin{aligned}
& \text { When } \theta=0 \text {, we have the Alfven and sound waves. } \\
& \text { If } v_{A} \gg c_{S} \text {, then the compressibility effects are }
\end{aligned}
$$
not very large, the two hydromagnetic modes are $v_{A} \cos \theta$, $\mathrm{c}_{\mathbf{s}} \cos \theta$, anisotropic in direction and the mode which propagates due to compressibility is $v_{A}\left(1-c_{S}^{2} \cos ^{2} \theta / v_{A}^{2}\right)$ not effected much by the direction. 
But if $\mathrm{c}_{\mathrm{S}} / \mathrm{v}_{\mathrm{A}}$ is not small, we no longer have a disturbance transmitted along the lines of force as a coherent whole, unless the motion is wholly normal to ${\underset{\sim}{\mathrm{B}}}_{0}$.

\subsection{Coriolis Force Effects}

In the presence of the Coriolis force $2 \rho \underset{\sim}{v} \times \Omega$; where $\Omega$ is the uniform angular velocity directed along the magnetic field direction, the dispersion relation for Alfvén waves assumes the form

$$
v_{p h}^{4}\left(1-\frac{4 \Omega^{2}}{\omega^{2}}\right)-2 v_{p h}^{2} v_{A}^{2}+v_{A}^{4}=0
$$

where

$$
\mathrm{v}_{\mathrm{ph}}^{2}=\omega^{2} / \mathrm{k}^{2}
$$

The two modes of propagation are

$$
v_{\mathrm{ph}}^{2}=\mathrm{v}_{\mathrm{A}}^{2}\left(1 \pm \frac{2 \Omega}{\omega}\right)^{-1}
$$

known as 'inertial' or 'fast' mode and the 'magnetic' or 'slow' mode.

Thus, in the presence of Coriolis force a plane Alfvén wave splits into two circularly polarized transverse waves with velocities $\mathrm{v}_{\mathrm{phs}}<\mathrm{v}_{\mathrm{A}}<\mathrm{v}_{\mathrm{phf}}$. It can be easily shown that there is no equi-partition between the kinetic and the magnetic energies (Lehnert, 1954a). 
1.8. Cylindrical Geometry: Torsional Alfvén Wave

Most of the experiments utilize cylindrical

containers in the laboratory. To understand the theory of these experiments we consider the Alfvén wave propagation in cylindrical geometry for incompressible and finitely conducting fluid.

Taking magnetic field in the z-direction and considering axisymmetric perturbations so that $\frac{\partial}{\partial \theta}=0$, we have

$$
\begin{aligned}
& \mathrm{v}_{\mathrm{r}}=0, \mathrm{v}_{\theta}=\mathrm{v}, \mathrm{v}_{\mathrm{z}}=0 \\
& \mathrm{~B}_{\mathrm{r}}=0, \mathrm{~B}_{\theta}=\mathrm{B}, \mathrm{B}_{\mathrm{z}}=0 .
\end{aligned}
$$

The equation for $B$ is given by

$$
\frac{\partial^{2} B}{\partial r^{2}}+\frac{1}{r} \frac{\partial B}{\partial r}+\left(k_{C}^{2}-\frac{1}{r^{2}}\right) B=0,
$$

where

$$
k_{c}^{2}=-\left[k^{2}+\frac{\left(\omega^{2}-k^{2} v_{A}^{2}\right) \mu_{0}}{i \omega n}\right] ;
$$

and $n\left(=\sigma^{-1}\right)$ is the resistivity.

Solution of $B$ which is finite at $r=0$ is given as

$$
B=A_{1}\left(k_{C} r\right) e^{i(k i-\omega t)},
$$

where $J_{1}\left(k_{c} r\right)$ is the Bessel function of the first order. The field variables are: 


$$
\begin{gathered}
v=-\frac{B_{0}^{k}}{\mu_{0} \rho \omega} \quad B, \quad J_{r}=-\frac{i k}{\mu_{0}} B, \quad J_{z}=\frac{k_{c}}{\mu_{0}} \frac{j_{0}(k c)}{J_{1}\left(k_{c} r\right)} B, \\
E_{r}=\frac{k}{\omega}\left(v_{A}^{2}-\frac{i \omega \eta}{\mu_{0}}\right) B \quad E_{z}=\frac{\eta}{\mu_{0}} k_{C} \frac{J_{0}(k r)}{J_{1}(k r)} B .
\end{gathered}
$$

In the absence of resistivity, the electric

field is entirely radial. The Alfvén wave becomes a torsional or shear wave. Adjacent magnetic surfaces are able to shear past each other without mutual coupling in their motion. If we take $k=k_{r}+i / \ell$, where $k$ is the propagation constant and $\ell$ the attenuation distance, $\ell$ is given by

$$
\ell=\frac{\mu_{0} 2 k_{r}\left[v_{A}^{4}+\omega^{2} \eta^{2} / \mu_{0}^{2}\right]}{\omega n\left[\omega^{2}+k_{C}^{2} v_{A}^{2}\right]}
$$

$k_{c}$ will be determined by the boundary conditions.

For example if we take a liquid in the cylinder with radius $R$ and the height $L$ and at the bottom $z=0$ excite a motion, $v(r, t)=\frac{v_{0} r}{R} e^{-i \omega t}$, the boundary conditions are:

$$
\begin{aligned}
& \text { i) } r=R, J_{r}=0, \\
& \text { ii) } z=0, v=v_{0} \frac{r}{R} e^{-i \omega t}
\end{aligned}
$$

and

$$
\text { iii) } z=L, J_{z}=0
$$


The first condition gives the wave numbers for different modes of propagation as $J_{1}\left(k_{c n} r\right)=0$, i.e., $k_{\mathrm{cn}}$ is given by the zeros of the Bessel function. The corresponding $k_{n}$ are then given by the Eq. (1.17).

Conditions (ii) and (iii) may be satisfied as follows: Consider the repeated reflections of the waves. A wave traveling up to the surface is reflected without phase difference in $\mathrm{v}$ but $\mathrm{h}$ is in the opposite phase in the upward and the downward wave. At the bottom, the conditions for $h$ and $\mathrm{v}$ are reversed. At a point $\mathrm{z}$ adding all the waves traveling up and down, i.e.,

$$
v(z)=v(0)\left[e^{i k z}+e^{i k(2 L-z)}+e^{-i k(2 L+z)}+e^{-i k(4 L-z)}+\ldots\right],
$$

the solution becomes

$$
v(r, z, t)=\sum_{n} A_{n} J_{1}\left(k_{c n} r\right) \frac{\cos k(L-z)}{\cos k L} e^{-i \omega t}
$$

For $z=0$ and $r<R$ we have

$$
\frac{v_{0} r}{R}=\sum_{n=1}^{\infty} A_{n} J_{1}\left(k_{c n} r\right)
$$

from which $A_{n}$ may be determined. Finally, the expression for $v(r, z)$ valid for $0 \leq v<R$ and $0 \leq z \leq L$ is

$$
\frac{v(r, z)}{v(r, 0)}=\sum_{n=1}^{\infty} \frac{2 J_{1}\left(k_{c n} r\right)}{k_{c n} r J_{2}\left(k_{c n} R\right)} \frac{\cos k_{n}(L-z)}{\cos k_{n} L}
$$

The above analysis was given by Lundquist (1949a) while studying the Alfvên waves experimentally. 


\subsection{Experimental Studies}

The concept of hydromagnetic waves was introduced by Alfvén in 1942 but the first experimental observation in the laboratory was made only in 1949, seven years later. This was mostly because of the difficulty in obtaining conditions under which the wave attenuation is sufficiently low to allow observation. The early experimental studies of Alfvén waves were conducted using liquid metals. Liquid mercury was used by Lundquist (1949b) and liquid sodium by Lehnert (1954b). As more recent experiments in plasmas, as will be discussed in the second chapter, are very similar to these early experiments we shall give a brief account of these here.

The mercury in a cylindrical vessel was placed between the pole pieces of an electromagnet with the axis of the cylinder aligned parallel to the direction of the field. The cylinder diameter was $15 \mathrm{~cm}$, the height $15 \mathrm{~cm}$ and the magnetic field about $10^{4}$ Gauss. These parameters satisfy the condition $\mathrm{L}>\mathrm{l}$. A circular disc was placed at the base of the vessel so as to be free to oscillate in its plane about the axis of the cylinder.

The oscillations of the disc do not produce any motion of the mercury in the absence of the magnetic field because of the inertia of the mercury. But, as soon as the magnetic field is switched on the motion of the disc 
interacts with the mercury electromagnetically and its oscillations were transmitted along the magnetic field. This is in fact, the propagation of hydromagnetic waves which in this case, are torsional waves along a cylinder. These waves as they reach the surface produce a rotational motion. This could be detected by means of a mirror floated suitably on the surface. The deflections of the light reflected from the mirror would indicate the rotational motion at the surface. Very low frequencies, less than one cycle per second, were used in order to keep the damping at a low level. Hence it was not possible to obtain any standing wave effects. The waves here will, obviously, be standing for $\lambda=z / 4$ and the amplitude quite large. But due to the damping effect, which originates from the finiteness of the conductivity of mercury the amplitude on the surface is much decreased.

Lehnert repeated the experiment with sodium, because. its higher electrical conductivity and lower density are more suitable for MHD experiments (the Lundquist number is 35 times than in the mercury), but the experiment is more difficult to perform because of the nature of sodium. As it is more easily oxidized, it is enclosed in a vessel in an atmosphere of some inert gas. To observe the motion of the surface in hydromagnetic oscillation, use is made of the probes which are held at various points on the surface. The motion of the material carrying "frozen-in" lines of 
force with it, induces in the probe an electric field given by

$$
\underset{\sim}{E}=-\underset{\sim}{\mathbf{v}} \times \underset{\sim}{\mathrm{B}}
$$

The relative magnitudes of these electric fields induced in different probes give at any instant the relative motions of the surface elements, hence the oscillation of the surface.

The experimental results agree qualitatively with the theory. 
REFERENCES OF CHAPTER I - 1

Alfvên, H., Existerice of Electromagnetic-Hydrodynamic Waves; Nature, 150, 405 (1942).

Ferraro, V. C. A., on the reflection and refraction of Alfvén Waves, Ap. J. 119, 393 (1954).

Lehnert, B., Magnetohydrodynamic waves under the action of the Coriolis force, Ap. J. 119, 647 (1954a).

Lehnert, B., Magnetohydrodynamic waves in liquid sodium, Phys. Rev. 94, 815 (1954b).

Lundquist, S., Experimental investigations of magnetohydrodynamic waves, Phys. Rev. 76,1805 (1949a). Lundquist, S., Experimental demonstration of magnetohydrodynamic waves, Nature, 164, 145 (1949b). 
OTHER REFERENCES OF CHAPTER I -1

Alfvén, H., Cosmical Electrodynamics, New York: Oxford University Press, 1950.

Amte, P. G., Dual Alfvén waves in superfluids with high electrical conductivity, Indian J. Phys. $\underline{48}$, 843-5 (1974). Cowling, T. G., Magnetohydrodynamics, New York: Inter- , science Publishers Inc., 1957.

Cramer, N. F., Lehane, J. A., May, R. M., Paoloni, F. J., Compressional Alfvén wave reflection from a magnetic 'cliff', Phys. Letters A (Netherlands) 32.A 307-8 (1970). Herlofson, N., Magnetohydrodynamic waves in a compressible fluid conductor, Nature, 165, 1020 (1950).

Lundquist, S., Studies in magnetohydrodynamics, Ark. Fys. 5, 297 (1952).

Narasimha, M. S., The influence of Coriolis force on MHD waves, Proc. Indian Acad. Sci., A 18, 202-7 (1973). Novikov, I. I., Kutnik, A. E., Analogue of the Alfvén velocity associated with the translation and rotation of an ideal infinitely conducting liquid, Fiz. and Khim. Obrab. Mater. (USSR) $4,32-4$. (1974).

Roberts, P. H., On the reflection and refraction of hydrodynamic waves, Ap. J. 121, 720 (1955).

Shah, S. K. D., Srivastava, K. M., Hydromagnetic waves in an infinitely conducting compressible fluid with an Inhomogeneous magnetic field of constant direction, Nuovo Cimento (B) (Italy) 63, 105-14, (1969). 


\section{$\underline{O R-I-2}$}

Stein, R. F., Reflection, refraction and coupling of magnetohydrodynamic waves at a density step, Ap. J. Supplement Series, 22, 419 (1971): 
REFERENCES FOR ALFVÉN WAVES IN

ASTROPHYSICS - 1

Beckers, J. M., The flux of Alfvén waves in sunspots, Astrophys. J. 203, 739-52 (1976).

Belcher, J. W., Olbert, S., Stellar winds driven by Alfvén waves, Astrophys. J. 200, 369-82 (1975).

Belcher, J. W., Solodyna, C. V., Alfvén waves and directional discontinuities in the interplanetary medium, J. Geophys. Res. . $80,181-6$ (1975).

Belcher, J. W., Burchsted, R., Energy.densities of Alfvén waves between 0.7 and $1.6 \mathrm{AU}, \mathrm{J}$. Geophys. Res. 79, 4765-8 (1974).

Belcher, J. W., Davis, L.,Jr., smith, E. J., Large-amplitude Alfvén waves in the interplanetary medium, J. Geophys. Res. $74,2303-8$ (1969).

Burlaga, L. F., Turner, J. M., Microscale 'Alfvén waves' in the solar wind at $1 \mathrm{AU}, J$. Geophys. Res. 81, 73-7 (1976). Cummings, W. D., countee, C., Lyons, D. . Wiley, W., The dominant mode of standing Alfvén waves at the synchronous orbit, J. Geophys. Res. 80, 3705-8 (1975).

Davydov, V.M., Diffraction of low-frequency electromagnetic waves on a stratified earth surrounded by a thin gyrotropic ionosphere, Geomagn. and Aeron. I1 845-51. (1971).

Dwarkin, M. L., Zmuda, A. J., Radford, W. E., Hydromagnetic waves at 6.25 earth radii with periods between 3 and 240 seconds, J. Geophys. Res. 76, 3668-74 (1.971). 
REFERENCES FOR ALFVÉN WAVES IN ASTROPHYSICS - 2

Hollweg, J. V., Alfvén waves in a two-fluid model of the solar wind, Astrophys. J. 181, 547-66 (1973).

Hollweg, J. V:, Improved limit on photon rest mass, Phys. Rev. Lett. 32, 961-2 (1974).

Hollweg, J. V., Transverse Alfvén waves in the solar wind arbitrary K, J. Geophys. Res. 79, 1539-41. (1974) .

Hollweg, J. V., Supergranulation-driven Alfvén waves in the solar chromosphere and related phenomena, Cosmic Electrodyn. (Netherlans) 2, 423-44. (1972).

Holmes, J. A., The confinement of galactic cosmic rays by Alfvên waves, Astron. Soc. (GB) 170, 251-60 (1975).

Ioffe, Z. M., On the mechanism of Alfvén wave generation in comets, Astron Zh, (USSR) 46, 1328-9 (1969).

Lanzerotti, L. J., Magnetohydrodynamic waves in the magnetosphere and the photon rest mass, Geophys. Res. Lett $1,229-30$ (1974). Mullan, D. J., Sunspot models with. Alfvén wave emission, Astrophys. J. 187, 621-31 (1974).

Owens, A. J., A new test for Alfvén waves in interplanetary space, Astrophys. and Space Sci., (Netherlands) 38, 469-7.3 (1975).

Piddington, J. H., The Alfvén-wave theory of solar flares, Sol. Phys. (Netherlands) 38, 465-81 (1974).

Rađoski, H. R., The polarization of Alfvén Waves, J. Geomagn. Geoelect. 285-7 (1968). 
REFERENCES FOR ALFVÉN WAVES IN

ASTROPHYSICS - 3

Richter, A. K., Wave-trains in the solar wind. II: Comments on the propagation of Alfven waves in the quiet interplanetary medium, Astrophys. and Space Sci. (Netherlands) 26, 95-105 (1974).

Skilling, J., Cosmic ray streaming. I. Effect of Alfvén

- waves on particles, stron Soc. (GB) 172; 557-66 (1975).

Toptygin, I. N., Magneto-Hydrodynamic waves in plasma of solar wind, Izv. vuz radiofiz. (USSR) 16, 971-80 (1973).

Uchida, Y., Sakurai, T., Oscillations in sunspot umbras due to trapped Alfvén waves excited by overstability, Astron. Soc. Japan, 27, 259-74 (1975).

Vinogradov, P. A., Parkhomov, V. A., MHD waves in the solar wind.A possible source of geomagnetic PC 3 pulsations, Geomagn. and Aeron: (USSR) 15, 134-7 (1975); Geomagn. and Aeron. 15, 109-11 (1975) .

Volk, H. J., Aplers, W., The Propagation of Alfvén waves and their directional anisotropy in the solar wind, Astrophys. and Space Sci. (Netherlands) 20, 267-85 (1973).

Whang, Y. C., Alfvén waves in spiral interplanetary field, J. Geophys. Res. 78, 7221-8 (1973).

Wilson P. R., Alfvén waves and Meyer's sunspot model, Astron. Soc. (GB) $172,535-43$ (1975). 


\section{REFERENCES FOR ALFVEN WAVES \\ IN SOLIDS - 1}

Ashkinadze, B. M. Altukov, P. D. , Magnetoplasma effects in optically excited GE and SI, Fiz. Tverdogo Tela (USSR) 17, 1004-11 (1975) Sov. Phys.-Solid state 17, 634-7.

Baynham, A. C. Boardman, A. D., Plasma effects in semiconductors' helicon and Alfvén waves, Taylor and Francis, London, England, 1971 .

Beletskii, N. N., Tetervov, A. P., Yakovenko, V. M., Nonpotential surface waves in magnetically active semiconductor plasma, Sov. Phys.-Semicond. 6 , 1807-10 (1973).

Caille, A., Wallace, P. R., Interaction of Magnon and Alfvén waves in itinerant antiferromagnetic chromium II, Solid state Commun. 9 , 1437-41 (1971).

Caille, A., Wallace, P. R., Solid State Commun. 7, 1283-6, (1969).

Chen, F. C., Kirschbaum, J., Kao, Y. H., Temperature dependence of Alfvén-wave amplitudes and carrier relation times in bismuth, Phys. Rev. B 6, 327-33 (1972).

Fahidy, T. Z., Wave phenomena in magnetoelectrolytic systems, Electrochim. Acta (GB) 21, 21-4 (1976).

Guthmann, C., D'Haenens, J. P., Libchaber, A., Effect of anisotropy and Landau damping in a geometry where $Q$ is not perpendicular to B, Phys. Rev. B 8, 561-70 (1973). 


\section{REFERENCES FOR ALFVEN WAVES}

IN SOLIDS - 2

Hermann, R.; Baune, W. ; and Kuka, G., Al fvén wa ves cyclotron resonance in semimentallic Bi-SB. Phys. Status Solidi B (Gerinany) 61; K77-80 (1974).

Hess, H.D. and Hinsch, H., Propagation of alfvén in bismuth. Appl. Phys. 2, 27-30 (1973).

Hu, P.; Narayanamurti, V.; and Dynes, R.C., Metals using nanosecond pulses of laser light case of alfvén waves in bismuth. Phys. Rev. Lett. 34, 1015-18 (1975).

Issacson, R.T. and Williams, G.A., Oscillations of the Fermi surface of bismuth. Phys. Rev. 185, 682-8 (1969).

Isaacson, R.T. and Williams, G.A., Al fvén-wave propagation in solid-state plasmas, II. Bismuth. Phys. Rev. 177, $738-46$ (1969) .

Kaner, E.A. and Skobov, V.G., Plasma effects in metals' helicon, and alfvén waves. Taylor and Franics, 1971. Krauss, A.R. and Furdyna, J.K., Al fvén-wave propagation and damping in pyrolytic and single-crystal graphite. Phys. Rev. B 7, 2520-39 (1973). Marston, E.H. and Kao, Y.H., Damped al fvén' waves in bismuth'. A determination of charge-carrier relaxation times. Phys. Rev. 182, 504-10 (1969).

Nakahara, J.; Kawamura, H.; and Sawada, Y., Interaction of alfvén waves with Bernstein modes in bismuth. Phys. Lett. 31A, 271-2 (1970). 
REFERENCES FOR ALFVÉN WAVES

IN SOLIDS

Platzman, P.M. and Wolff, P.A., Waves and Interactions in

Solid State Plasmas, Academic Press, New York, 1973 .

Schwarz, B. and Hinsch,.H., Magnetoplasma waves in anisotropic semiconductors and semimetals. (Al fvên waves in bismuth). Appl. Phys. 10, 325-30 (1976).

Suematsu, H. and Tanuma, S., Alfvén wave propagation in atimony. J. Phys. Soc. Jap. 41, 496-501 (1976). Vaselago, V.G.; Glushkov, M.V.; and Lyn'ko, L.V., Propagation of magnetoplasma waves in $\mathrm{Bi}$ alloyed with l'e. JETP Lett. $13,247-9$ (1971).

Wilson, P.R., Al fyén waves and the sunspot phenomenon. Sol. Phys. $42,333-40$ (1975). 


\section{$!$ \\ II. ALFVEN WAVES IN PLASMAS}

\subsection{Introduction}

It is now well known that MHD or ideal Alfvén wave

is a fundamental mode of wave propagation in plasmas. involving low frequency ion oscillations in the presence of a magnetic field.

At first sight, it appears surprising that a wave propagating in an incompressible and infinitely conducting media can exist in an ionized gas where equations $\nabla \cdot \mathrm{v}=0$ and $\underset{\sim}{\mathrm{E}}+\underset{\sim}{\mathrm{v}} \times \underset{\sim}{\mathrm{B}} \approx 0$ hold. As pointed out by Herlofson in 1950, these difficulties are more formal than real. Alfvén wave exists in plasmas for very low frequencies $\omega \ll \omega_{c i}$. Hence, the equation of motion for plasmas

$$
\frac{d v}{d \underline{v}}=\frac{e}{m}(\underset{\sim}{E}+\underset{\sim}{v} \times \underset{\sim}{B})
$$

at low frequencies becomes equivalent to the infinite conductivity assumption in a liquid. Actually this characteristic condition means that the magnetic flux through a surface moving with the liquid. is independent of time. In both cases the magnetic lines of force will therefore move with the plasma: Concerning the incompressibility condition, 
the Alfven wave perturbations are such that the velocity of the medium is always perpendicular to the wave normal and so do not compres's the plasma. Therefore, $\nabla \cdot \underset{\sim}{v}=0$ is satisfied without suggesting that the plasma is incompressible.

\subsection{Dispersion Relation}

We shall use the two fluid descriptions for the plasma in order to understand the electric field and current which are set up in the plasma with the propagation of the Alfvén wave.

Since the perturbations do not compress the plasma, the plasma can be treated as 'cold'. The equivalent dielectric tensor derived from the two-fluid model for a cold plasma is:

$$
\underset{\approx}{K}=\left(\begin{array}{rrr}
s & -i D & 0 \\
i D & s & 0 \\
0 & 0 & P
\end{array}\right),
$$

with

$$
\begin{aligned}
& S=\frac{1}{2}(R+L), \quad D=\frac{1}{2}(R-L), \\
& R=1-\frac{\omega_{p e}^{2}}{\omega\left(\omega-\omega_{c e}\right)}-\frac{\omega_{p i}^{2}}{\omega\left(\omega+\omega_{c i}\right)}, \\
& L=1-\frac{\omega_{p e}^{2}}{\omega\left(\omega+\omega_{c e}\right)}-\frac{\omega_{p i}^{2}}{\omega\left(\omega-\omega_{c i}\right)}, \\
& \therefore \quad \therefore \quad . .
\end{aligned}
$$

The notations are standard and are listed in the Appendix. 
The dispersion relation for the waves propagating at an angle $\theta$ with respect to the magnetic field $B_{0}=\left(0,0, B_{0}\right)$ direction is given by

$$
A n^{4}-B n^{2}+C=0
$$

where

$$
\left.\begin{array}{l}
A=S \sin ^{2} \theta+P \cos ^{2} \theta \\
B=\text { RL } \sin ^{2} \theta+P S\left(1+\cos ^{2} \theta\right) \\
C=\text { PRL. }
\end{array}\right\}
$$

where $\mathrm{n}=\mathrm{ck} / \omega$ is the index of refraction. When $\theta=0$, the two transverse modes are

$$
\mathrm{n}^{2}=\mathrm{R}, \quad \mathrm{n}^{2}=\mathrm{L}
$$

These are circularly polarized modes. In the limit $\omega \ll \omega_{\mathrm{Ci}}$ ' we find

$$
\mathrm{n}^{2}=\mathrm{R}=\mathrm{L} \simeq 1+\frac{\rho \mu_{0} c^{2}}{\mathrm{~B}^{2}}
$$

where $\rho=\mathrm{n}_{0} \mathrm{~m}_{i}$. The two modes, shear and compressional Alfvén waves are in degenerate at $\theta=0$.

$$
\text { Fruili (2.10), we find }
$$

$$
\omega^{2} / k^{2}=\frac{c^{2}}{1+c^{2} / v_{A}^{2}} \simeq v_{A}^{2}
$$

with $v_{A}^{2} \ll c^{2}$. Since $(2.10)$ gives the dielectric constant for a low frequency wave the Alfvén wave can be treated as an electromagnetic wave which is modified by the high dielectric constant of the medium. 
The electric field is in the $x$-direction. The velocity components are then given as:

$$
\begin{aligned}
& v_{i x}=\frac{i e}{m_{i} \omega}\left(1-\frac{\omega_{c i}^{2}}{\omega^{2}}\right)^{-1} E \\
& v_{i y}=\frac{e_{c i}^{\omega}}{m_{i} \omega^{2}}\left(1-\frac{\omega_{C i}^{2}}{\omega^{2}}\right)^{-1} E .
\end{aligned}
$$

The corresponding solution of the electron equation of motion gives in the limit $\omega^{2} \ll \omega_{c e}^{2}$,

$$
\begin{aligned}
& v_{e x} \rightarrow 0 \\
& v_{e y}=-\frac{E}{B_{0}} .
\end{aligned}
$$

The fluctuating magnetic field $B_{y}$, is given by the Maxwell equation $\underset{\sim}{\nabla \times E}=-\underset{\sim}{\partial \dot{B}} / \partial t$, as

$$
E=\frac{\omega}{k} B_{Y}
$$

Thus, we have $\underset{\sim}{\mathrm{E}}$ in the $\mathrm{x}$-direction, $\underset{\sim}{\mathrm{B}}$ in the $\mathrm{z}$-direction. which gives $\underset{\sim}{\mathrm{E}} \times \mathrm{\sim}_{0}^{\mathrm{B}}$ drift in the $\mathrm{y}$-direction, which for $\omega^{2} \ll \omega_{c i}^{2}$ is same for both ions and electrons. Thus the motion of the fluid in the $y$-direction has magnitude of the velocity $\left|\mathrm{E} / \mathrm{B}_{0}\right|$. The ripples in the magnetic field lines of force move with the velocity $(\omega / \mathrm{k})\left|\mathrm{B}_{\mathrm{y}} / \mathrm{B}_{0}\right|$, which is equal to fluid velocity. The plasma particles thus 
bound to the magnetic lines of force provide an added inertial effect which lowers the frequency of an electromagnetic oscillation of the given wavelength. The principal mode induces a shear of the magnetic lines but no compression (i.e., magnitude of the field does not change in the first order). The restoring force is thus derived solely from the tension (i.e., curvature) of the magnetic lines. The string analogy as in the conducting liquid case holds for ionized media also. But, it should be noted that concept of plasma frozen to lines of force and moving with them is an accurate one as long as there is no electric field along $\underset{\sim}{\mathrm{B}} 0$.

What sustains the electric field which was presupposed to be there? As $\underset{\sim}{\mathrm{E}}$ fluctuates, the ion inertia causes ions to lag behind the electrons which induces the polarization drift $\underset{\sim p}{v}$ in the direction of $\underset{\sim}{E}$. This drift $v_{\text {ix }}$ given by Eq.(2.12) causes a current $\underset{\sim}{J}$ to flow in the $x$-direction. The resulting $\underset{\sim}{J} \underset{\sim}{\times B}$ force on the fluid is in the $y$-direction and is $90^{\circ}$ out of phase with the velocity v. This force perpetuates the oscillation in the same way as in any oscillator where the force is out of phase with the velocity. It is, of course, always the ion inertia that causes an overshoot and sustains the oscillation, but in a plasma the momentum is transferred in a complicated way via the electromagnetic forces. 
2.3 Compressional Alfvén Wave

In Eq. (2.7) when $\theta=\pi / 2$, the dispersion

equation gives

$$
n^{2}=\frac{R L}{S} \simeq 1+\frac{\rho \mu_{0} c^{2}}{B_{0}^{2}} \text { when } \omega \ll \omega_{C i}
$$

In this case, the wave propagates perpendicular to the magnetic lines of force and it is the compression of the magnetic lines which contributes to the restoring force along with the curvature.

The (shear or torsional) Alfvén wave has no magnetic field induced in the longitudinal direction, while the compressional Alfvén mode does have a component $B$ parallel to $B_{0}$ in general

Considering $\theta$, not in the vicinity of $\pi / 2$ we get

$$
n^{2} \simeq 1+\frac{\rho \mu_{0} c^{2}}{B_{0}^{2}} \text {. }
$$

and

$$
\mathrm{n}^{2} \cos ^{2} \theta \simeq 1+\frac{\rho \mu_{0} c^{2}}{\mathrm{~B}_{0}^{2}}
$$

The first isotropic mode is the compressional mode and the second is a torsional or shear moce. This can be 
found out by calculating $\underset{\sim}{\mathrm{E}}$, which shows that $\mathrm{E}_{\mathrm{z}} \approx 0$ for both the modes, whereas $E_{X} \approx 0$ for $(2.18)$ and $E_{Y} \approx 0$ for (2.19). From the relation

$$
\underset{\sim}{\mathrm{E}}+\underset{\sim}{\mathrm{v}} \times \underset{\sim}{\mathrm{B}}=0 \text {, }
$$

we get

$$
\underline{\sim} \sim \hat{z} \cos \left(k_{x} x+k_{z} z-\omega t\right)
$$

for the mode (2.18) and

$$
\underset{\sim}{v} \hat{y} \cos \left(k_{x} x+k_{z} z-\omega t\right)
$$

for the mode (2.19), which confirm the compressional and torsional nature of these modes.

2.4 Hall Current Effects: Finite Frequency Alfvén wave.

The Hall current effect becomes more important than the electrical resistivity whenever the magnetic field $\underset{\sim}{\mathrm{B}}$ is so large that the gyrofrequency of electrons greatly exceeds their collision frequency. This can happen in high temperature low density plasma for reasonable strength of the magnetic field.

$$
\begin{gathered}
\text { When } \omega \ll \omega_{C e} \text {, but } \omega<\omega_{C i} \text { then } \\
R \simeq \frac{c^{2}}{v_{A}^{2}} \frac{\omega_{C i}}{\omega_{C i}+\omega} \quad \text { and } \quad I \simeq \frac{c^{2}}{v_{A}^{2}} \frac{\omega_{C i}}{\omega_{C i}-\omega} .
\end{gathered}
$$


These two modes give

$$
v_{\mathrm{ph}}^{2}=v_{\mathrm{A}}^{2}\left(1 \pm \omega / \omega_{\mathrm{ci}}\right)
$$

where + and - stand for compressional and shear Alfvén waves.

Hence, the effect of finite ion gyrofrequency

'disperses the Alfvén wave and the unidirectional property which allows the 'ideal' Alfven wave to propagate without attenuation. is not met in the case of a 'finite frequency' Alfvén wave.

When the Coriolis force is taken into account in addition, an interesting effect appears (Uberoi, 1976). The presence of rotation and Hail effects gives rise to a critical frequency, $\omega_{\mathrm{Cr}}$, lying between the rotational and ion-cyclotron frequencies,

$$
\omega_{c r}^{2}=2 \Omega\left(v_{A}^{2} / \lambda+2 \Omega\right),
$$

where $\lambda=\mathrm{B}_{0} / 4 \pi n e$, at which the slow and fast modes have the same phase velocity. Across this frequency both modes undergo polarization reversal. At this critical frequency conditions may exist for strong coupling between the two modes of propagation.

2.5. Ion-Neutral Collision Effects

In a partially ionized plasma, the ion-neutral collisions can become very important.

The effect of neutral atoms is introduced through the collision term ${\underset{\sim}{p}}^{i n}$ in the momentum equation for the 
plasma ion. Since $m_{i} \approx m_{n}$

$$
\underline{P}^{i n}=n_{m} m_{n}\left(-v_{i}^{v}+v_{\sim n}\right) v_{n i}
$$

where $v_{n i}$ is the ion-neutral collision frequency. The velocity ${\underset{\sim}{\mathrm{n}}}_{\mathrm{n}}$ is determined by the equation

$$
\frac{\partial v_{n}}{\partial t}={\underset{\sim}{p}}^{n i}
$$

with

$$
\stackrel{\sim}{P}^{n i}=-{\underset{\sim}{P}}^{i n},
$$

The Alfvén velocity is then given by

$$
v_{A}=B_{0} /\left(\mu_{0} \rho_{1}\right)^{1 / 2},
$$

with

$$
\rho_{1}=\left[\rho_{i}+\rho_{n}(1+i \tau) /\left(1+\tau^{2}\right)\right],
$$

where $\tau=\omega / \nu_{n i}$.

2.6 Finite Larmour Radius Effects: Kinetic Alfvén Wave.

When the perpendicular wavelength, $2 \pi / k_{1}$, becomes comparable to the ion gyroradius, $\rho_{i}$,ions can no longer follow the magnetic line of force, while electrons are still attached to the field line because of their small Larmour radius. This produces charge separation and coupling to the 
electrostatic mode (Stepanov, 1958, Stéfant., 1970). To treat this effect we must use vlasov equations for ions. When $k \rho_{i} \approx 1$, the frequency of the fast (compressional) mode becomes the order of of the ion cyclotron frequency, hence we can consider that the fast mode is decoupled. Then the compressional component of the magnetic field perturbation $\mathrm{B}_{2}$ can be assumed to be much smaller than the transverse components. This allows us to use a scaliar potential $\phi$ to represent the transverse component of the electric field, $E_{1}$,

$$
\underset{\sim}{E}=-\nabla_{\perp} \phi
$$

because then $\mathrm{B}_{\mathrm{z}}$ becomes zero. To represent $\mathrm{z}$ component lof the electric field we must use a different potential, $\psi$,

$$
E_{z}=-\partial \psi / \partial z,
$$

so that the transverse components of $\underset{\sim}{\underset{\sim}{E}}$ are not zero. The appropriate field equations are Poisson's equation,

$$
\nabla_{\perp}^{2} \phi+\frac{\partial^{2} \psi}{\partial z^{2}}=\frac{e}{\varepsilon_{0}}\left(n_{i}-n_{e}\right) \simeq 0
$$

and the $z$ component of Ampere's law,

$$
\frac{\partial}{\partial z} \nabla_{1}^{2}(\phi-\psi)=\mu_{0} \frac{\partial}{\partial t}\left(J_{i z}+J_{e z}\right)
$$

$\mathrm{n}_{i}, \mathrm{n}_{\mathrm{e}}, \mathrm{J}_{i z}, \mathrm{~J}_{\mathrm{ez}}$ are obtained from Iinearized vlasov equation. (Appendix) of each species, jy ascuning tire unperturbed distribution function to be llazweilian (Rukhadze and Silin, 1969). 


$$
\begin{aligned}
& \frac{e_{i}}{\varepsilon_{0}}=-\frac{\omega_{\mathrm{p} i}^{2}}{v_{\mathrm{T} i}^{2}}\left[1-I_{0}\left(\lambda_{i}\right) e^{-\lambda_{i}}\right] \phi \\
& +\frac{\omega_{p i}^{2} k_{z}^{2}}{\omega^{2}} I_{0}\left(\lambda_{i}\right) e^{-\lambda}\left(1-i \delta_{i}\right) \psi, \\
& \frac{\mathrm{en}_{\mathrm{e}}}{\varepsilon_{0}}=\frac{\omega_{\mathrm{pe}}^{2}}{\mathrm{v}_{\mathrm{Te}}^{2}}\left(1+i \delta_{\mathrm{e}}\right) \psi, \\
& \mu_{0} J_{i z}=\frac{\omega_{p i}^{2}}{c^{2}} \frac{k_{z}}{\omega} I_{0}\left(\lambda_{i}\right) e^{-\lambda_{i}}\left(1-i \delta_{i}\right) \psi, \\
& \mu_{0} J_{e z}=-\frac{\omega_{p e}^{2}}{c^{2} v_{T e}^{2}} \frac{\omega}{k_{z}}\left(1+i \delta_{e}\right) \psi,
\end{aligned}
$$

where $\quad \lambda_{i}=k_{\perp}^{2} \rho_{i}^{2}, \quad I_{0}\left(\lambda_{i}\right)$ is the modified Bessel function of the first kind and $\delta_{i}$ and $\delta_{e}$ are the fractional Landau damping rates,

$$
\begin{aligned}
& \delta_{i}=2 \sqrt{\pi} \beta_{i}^{-3 / 2} \exp \left(-\beta_{i}^{-1}\right) \\
& \delta_{e}=\sqrt{\pi} \beta_{i}^{-1 / 2}\left(T_{i} / T_{e}\right)^{1 / 2}\left(m_{e} / m_{i}\right)^{1 / 2},
\end{aligned}
$$


and

$$
\beta_{i}=2 v_{T i}^{2} / v_{A}^{2}
$$

$v_{T e}$ and $v_{T i}$ are the electron and ion thermal speed. The dispersion relation can be obtained by substituting

Eqs. (2.35) to (2.38) into (2.33) and (2.34). If we ignore the damping,

$$
\begin{aligned}
& \left(I_{0} e^{-\lambda} i-\frac{\omega^{2}}{k_{z}^{2} c_{S}^{2}}\right)\left[1-\frac{\omega^{2}}{k_{z}^{2} v_{A}} \frac{1}{\lambda_{i}}\left(I-I_{0} e^{-\lambda}\right)\right] \\
= & \frac{\omega^{2}}{k_{z}^{2} v_{T i}^{2}}\left(I-I_{0} e^{-\lambda}{ }^{-\lambda}\right),
\end{aligned}
$$

where $c_{s}$ is the ion sound speed with electron temperature, $c_{S}^{2}=T_{e} / m_{i}$ : The above dispersion relation shows the coupling of the Alfven wave and the ion acoustic wave. In a low $\beta$ plasma, since $c_{s}>v_{s}$, they are decoupled and the dispersion relation of the Alfvén wave becomes

$$
\frac{\omega^{2}}{k_{z}^{2} v_{A}^{2}}=\frac{\lambda_{i}}{1-I_{0} e^{-\lambda_{i}}}+\frac{T_{e}}{T_{i}} \lambda_{i} .
$$

We call here the wave represented by this dispersion relation as "kinetic Alfvén wave", because of this kinetic property. If $\lambda_{i} \ll 1$, the dispersion relation of the kinetic Alfvén wave roduces to 


$$
\omega^{2}=k_{z}^{2} v_{A}^{2}\left[1+k_{1}^{2} \rho_{i}^{2}\left(3 / 4+T_{e} / T_{i}\right)\right] \cdot(2.44)
$$

The kinetic Alfver wave can propagate across the magnetic field and faces both electron and ion Landau damping because of its coupling to the electrostatic mode. It is also important to note the wave accompanies the electric field in the direction of the ambient magnetic field.

\subsection{Alfvén Waves in Cylindrical plasmas}

The theory of Alfvern wave in cylindrical geometries in Section 1.8 was developed to understand experiments, where only the torsional waves were excited. However, in a plasma wave guide, (a long electrically conducting, cylindrical tube containing a plasma confined by a uniform magnetic field directed along the axis), there can be many different modes of propagation. It therefore, becomes necessary to study the properties of Alfvén wave relative to other modes of propagation.

Under laboratory conditions it is usual to assume that the plasma pressure is negligible compared to the magnetic pressure.

The ideal MHD equations for axisymmetric perturbations $(m=0)$ of the type $\exp (-i \omega t+i k z)$ give the following three equations for the components of the magnetic field vector:

$$
\left(\mathrm{k}_{\mathrm{A}}^{2}-\mathrm{k}^{2} \mathrm{LB} \mathrm{B}_{0}=0,\right.
$$




$$
\begin{gathered}
\left(k_{A}^{2}-k^{2}\right)_{r}=i k \frac{d B}{d r}, \\
\frac{1}{r} \frac{d}{d r}\left(r \frac{d B_{z}}{d r}\right)+k_{A}^{2} B_{z}=i k \frac{1}{r} \frac{d\left(r B_{r}\right)}{d r} .
\end{gathered}
$$

The equations thus decouple to give the pure torsional and compressional Alfvén waves.

For the compressional mode $\mathrm{B}_{z}$ is governed by the equation

$$
\frac{d^{2} B_{z}}{d r^{2}}+\frac{1}{r} \frac{d B_{z}}{d r}+\left(k_{A}^{2}-k^{2}-\frac{l}{r^{2}}\right) B_{z}=0
$$

which gives

$$
B_{z}=A J_{1}\left(k_{C} r\right)
$$

where

$$
\mathrm{k}_{\mathrm{C}}^{2}=\left(\mathrm{k}_{\mathrm{A}}^{2}-\mathrm{k}^{2}\right) \text {. }
$$

The compressional modes therefore have a iower cutoff frequency, obtained by setting $k=0$ in $\mathrm{Eq} .(2.50)$,

$$
\omega_{\mathrm{OC}}=\mathrm{k}_{\mathrm{C}} \mathrm{v}_{\mathrm{A}}
$$

where $k_{c}$ is determined by the eigenvalue condition

$$
\mathrm{J}_{1}\left(k_{c}{ }^{R}\right)=0,
$$

which arises due to boundary conditions at $r=R$, the radius of the cylinder. 
For torsional or shear modes, the frequency is

determined by

$$
\omega=k v_{A}
$$

$B_{\theta}$ is an arbitrary function of $r$. These modes are therefore characterized by a constant phase velocity $v_{A}$ and by the absence of a cut-off. Since $R$ is not contained in (2.53), the walls have no effect. The Alfvén mode thus has a very special nature.

The compressional and shear Alfvén mode correspond to $\mathrm{TE}$ modes and principal modes respectively in the hydromagnetic wave guide theory, first discussed by Newcomb (1957).

The cut-off frequency $\omega_{\text {oc }}$ is the lowest frequency below which the compressional mode does not exist. In laboratory plasmas the frequently $\omega_{\text {oc }}$ is often comparable or is greater than the ion-cyclotron frequency. This makes the study of Alfvén wave excited well below the ion-cyclotron frequency simpler. For example, in the experiment of Wilcox et al. to be described in the next section, the Alfvén velocity $v_{A}=2 \cdot 8 \times 10^{7} \mathrm{~cm} / \mathrm{sec}$ for $B_{0}=10,000$ Gauss. Therefore $\omega_{C i} \simeq 10^{8} / \mathrm{sec}$ and $\omega_{\text {OC }}=\frac{3.83}{R} v_{A} \simeq 4 \times 10^{7}$ with $R=5 \mathrm{~cm}$. Hence $\omega_{\text {oc }}$ is comparable to $\omega_{\mathbf{C i}}$. Moving away from the ideal situation and taking Hall effect, finite conductivity and ion-neutral collisions into account, the general dispersion relation when $m \neq 0$ becomes (Woods, 1962) 


$$
\begin{aligned}
& \left\{s k^{2}-k_{A}^{2}\left(1+i \delta_{\perp} k^{2}+i \delta_{1} k_{c}^{2}\right)\right\}\left\{s\left(k^{2}+k_{C}^{2}\right)-k_{A}^{2} \cdot\left[1+i \delta_{\perp}\left(k^{2}+k_{c}^{2}\right)\right]\right\} \\
= & k^{2}\left(k^{2}+k_{c}^{2}\right) \Omega^{2}
\end{aligned}
$$

where

$$
s=\left(1+\frac{\rho_{n}}{\rho_{i}} \frac{1+i \tau}{1+\tau^{2}}\right), k_{A}=\omega / v_{A^{\prime}}, \quad \Omega=\omega / \omega_{C i}, \delta=\frac{1}{\mu_{0} \sigma \omega}
$$

The two modes in (2.54) which are now coupled represent 'slow' and 'fast' modes corresponding to torsional and compressional modes at low frequencies.

$$
\text { Let } \left.k=k_{r}+i / l \text {, then at low frequencies (2.5 } 1\right)
$$

gives

$$
k_{r}^{2}=\frac{k_{A}^{2}}{s_{1}}-\left\{\begin{array}{cl}
0 & \text { (slow) } \\
k_{c}^{2} & \text { (fast) }
\end{array}\right.
$$

and

$$
\quad \frac{1}{\ell}=\frac{k_{A}^{2}}{2 s_{1}^{2} k_{r}}\left(s_{2}+\delta_{\perp} k_{A}^{2}+\begin{array}{cc}
\delta_{11} s_{1} k_{c}^{2} & \text { (slow) } \\
0 & \text { (fast) })
\end{array}\right\}
$$

where $s \equiv s_{1}+i s_{2}$.

When $s_{1}=1$ and $s_{2}=0$, we get

$$
\ell=\frac{2 \mu_{0} k_{r} v_{A}^{2}}{\omega n\left(k_{A}^{2}+k_{C}^{2}\right)}
$$


for the slow wave which is same as Eq. (1.33) to lowest order in $\eta$, in Chapter $I$.

$$
\text { When } \delta=0 \text {, }
$$

$$
\ell=\frac{s_{2} v_{A}}{2 \omega s_{1}^{3 / 2}}
$$

for the slow wave. We note that the damping distance due to both neutral particle collision and conductivity is proportional to $\mathrm{B}_{0}$ for the torsional Alfvén mode.

- When $m \neq 0$, the components of the magnetic field vector associated with the slow and fast wave to first order in $\Omega$, are given respectively as:

$$
\begin{aligned}
& B_{r}=i C\left\{\frac{m}{k_{c}^{r}} J_{m}\left(k_{c} r\right)-\frac{k_{r}^{2} \Omega}{s_{1}\left(k_{r}^{2}+k_{c}^{2}\right)-k_{A}^{2}} J_{m}^{\prime}\left(k_{C} r\right)\right\} \\
& B_{\theta}=-C\left\{J_{m}^{\prime}\left(k_{c} r\right)-\frac{k_{r}^{2} m \Omega}{k_{c} r\left\{s_{1}\left(k_{r}^{2}+k_{c}^{2}\right)-k_{A}^{2}\right\}} J_{m}\left(k_{C} r\right)\right\} \\
& B_{z}=-c \frac{k_{r} \Omega}{s_{1}\left(k_{r}^{2}+k_{C}^{2}\right)-k_{A}^{2}} k_{C} J_{m}\left(k_{C} r\right) .
\end{aligned}
$$

and

$$
B_{r}=-i A\left\{\frac{m}{k_{c} r} \frac{k_{r}\left(k_{r}^{2}+k_{c}^{2}\right)}{s_{1} k_{r}^{2}-k_{A}^{2}} \Omega_{m}\left(k_{c} r\right)-k_{r} J_{m}^{\prime}\left(k_{c} r\right)\right\}
$$




$$
\left.\begin{array}{l}
B_{\theta}=A\left\{\frac{k_{r}\left(k_{r}^{2}+k_{c}^{2}\right) \Omega}{s_{1} k_{r}^{2}-k_{A}^{2}} J_{m}^{\prime}\left(k_{c} r\right)-\frac{k_{r} m}{k_{c} r} J_{m}\left(k_{c} r\right)\right\} \\
B_{z}=A k_{c} J_{m}\left(k_{c} r\right) .
\end{array}\right\}
$$

The boundary conditions give the following eigenvalue conditions:

$$
\begin{aligned}
m \neq 0, \quad J_{m}^{\prime}\left(k_{c} r\right) & =0 \quad \text { (fast wave), } \\
& J_{m}\left(k_{c} r\right)=0 \quad \text { (slow wave), }
\end{aligned}
$$

When $\mathrm{m}=0$, these become

$$
J_{0}^{\prime}\left(k_{c} r\right)=0, \text { for both waves. }
$$

The boundary conditions are for conducting walls.

But, for the case $m=0$, the torsional wave has the boundary condition (2.62) even in case of insulating walls.

We note from (2.59) that for low frequencies $\omega \ll \omega_{c i}$ and $m=0$, the slow wave is almost entirely a torsional wave. Hence, at low frequencies and for axisymmetrical perturbations, the torsional wave decouples from the compressional mode and if $\omega_{\text {oc }} \sim \omega_{C i}$, the Alfvén torsional mode is the only mode propagating in laboratory plasmas at very low frequencies. 


\subsection{Experimental Studies of Alfvén Wave Properties}

The experimental generation and detection of Alfvền

waves in a. magnetized plasma was first reported at Berkeley, California by Allen, Baker, Pyle and wilcox (1959) and by Jephcott. (1959) in England.

The experiments reported in plasma, though conceptually very similar to that conducted by Lehnert, allowed a detailed study of the properties of Alfven waves discussed in Chapter I. The major difficulty in these experiments was that the damping distance both due to ion-neutral collisions and finite conductivity was proportional to $\mathrm{B}_{0}$. To overcome the damping it was necessary to have very high magnetic fields of the order of $10 \mathrm{KG}$. With large $\mathrm{B}_{0}, \mathrm{v}_{\mathrm{A}}$ and hence the wavelength becomes uncomfortably large unless the density is high.

In the experiment of Wilcox et al. (1960, 1961) with $\mathrm{B}_{0}$ equal to $10 \mathrm{KG}$, a density of $6 \times 10^{15} / \mathrm{cm}^{3}$ was used to achieve a low Alfvén speed of $2.8 \times 10^{7} \mathrm{~cm} / \mathrm{sec}$. The work was done in a highly ionized hydrogen plasma created in a cylindrical copper tube immersed in a uniform axial magnetic field which could be varied in strength up to $16 \mathrm{~K}$ Gauss. The plasma was produced by an ionizing wave front in many respects similar to a 'switch-ion' shock wave. The plasma thus produced was initially 80 to 100 percent ionized, with an ion density greater than $5 \times 10^{5} / \mathrm{cm}^{3}$, and an estimated temperature around $10^{4}{ }^{\circ} \mathrm{K}$ Torsional hydromagnetic waves 


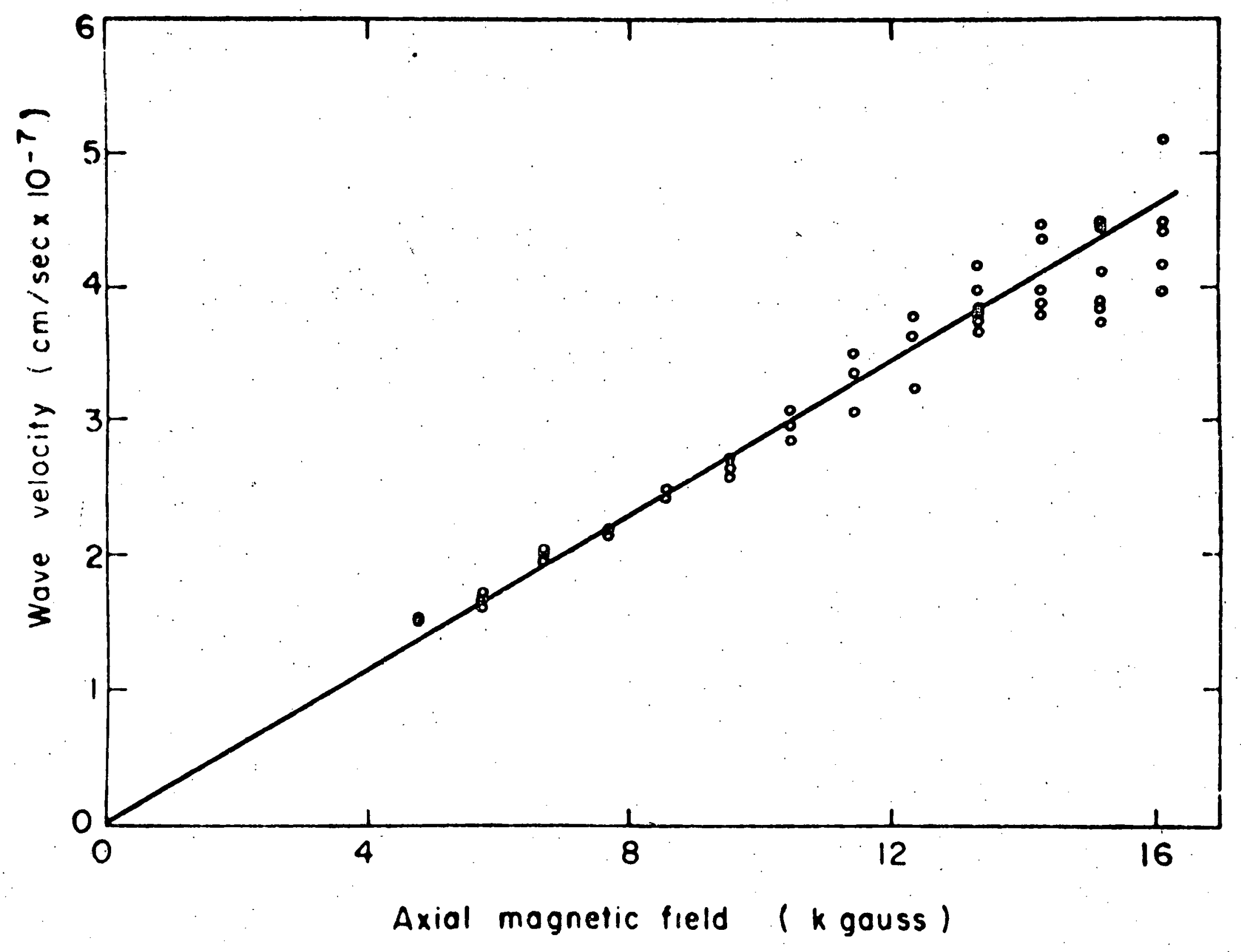




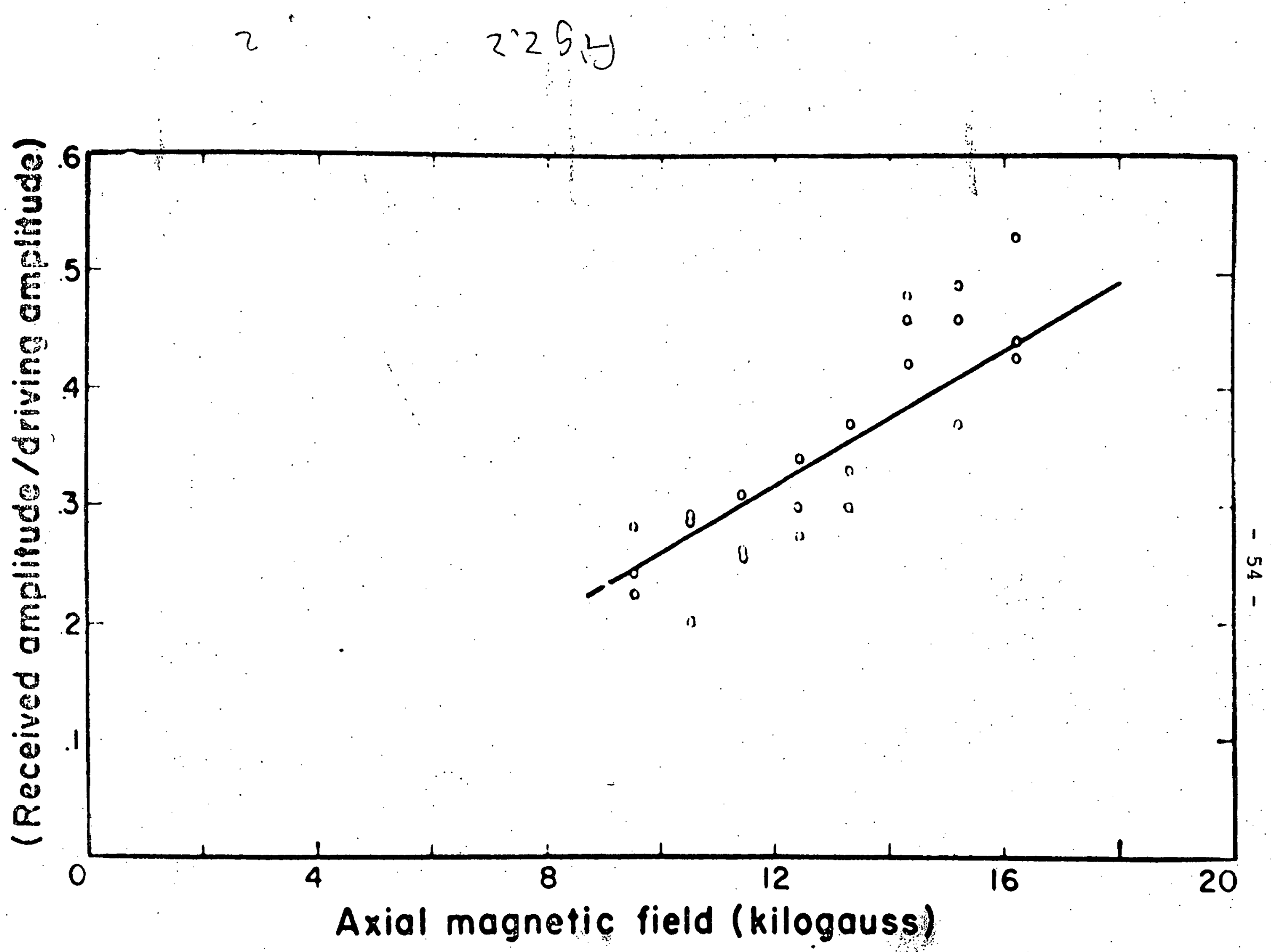


were induced in the plasma by discharging a small capacitor between the copper cylinder and a co-axial electrode mounted in an insulator at the end of the tube. The co-axial drivingelectrode system has the property of generating azimuthally symmetrical waves. The wave magnetic fields were observed with small magnetic probes, and the integrated wave electric field observed as a voltage on co-axial electrodes. Since, the theoretical analysis postulates an azimuthally symmetrical wave-propagation, care was taken to measure the azimuthal symmetry. It was measured experimentally with four magnetic probes disposed 90 degrees apart on the same base circle. The symmetry was found to be present within a few percent. The experimental results are shown in Figs. 2.1-2.6. Figure 2.1 shows measurements of phase velocity versus magnetic field depicting the linear dependence as given by Eq. (1.1) in Chapter I.

Figure 2.2 gives the attenuation against the magnetic field strength. The attenuation is mainly due to finite conductivity as the plasma is fully ionized. A good agreement between theory and experimental values is seen. The attenuation of Alfvén waves due to neutral damping was measured by Jephcott and Stocker (1962) and Brown (1965). The most important check on the theoretical predictions for the wave field was provided by the observation of wave reflections. The reflections were observed from an 


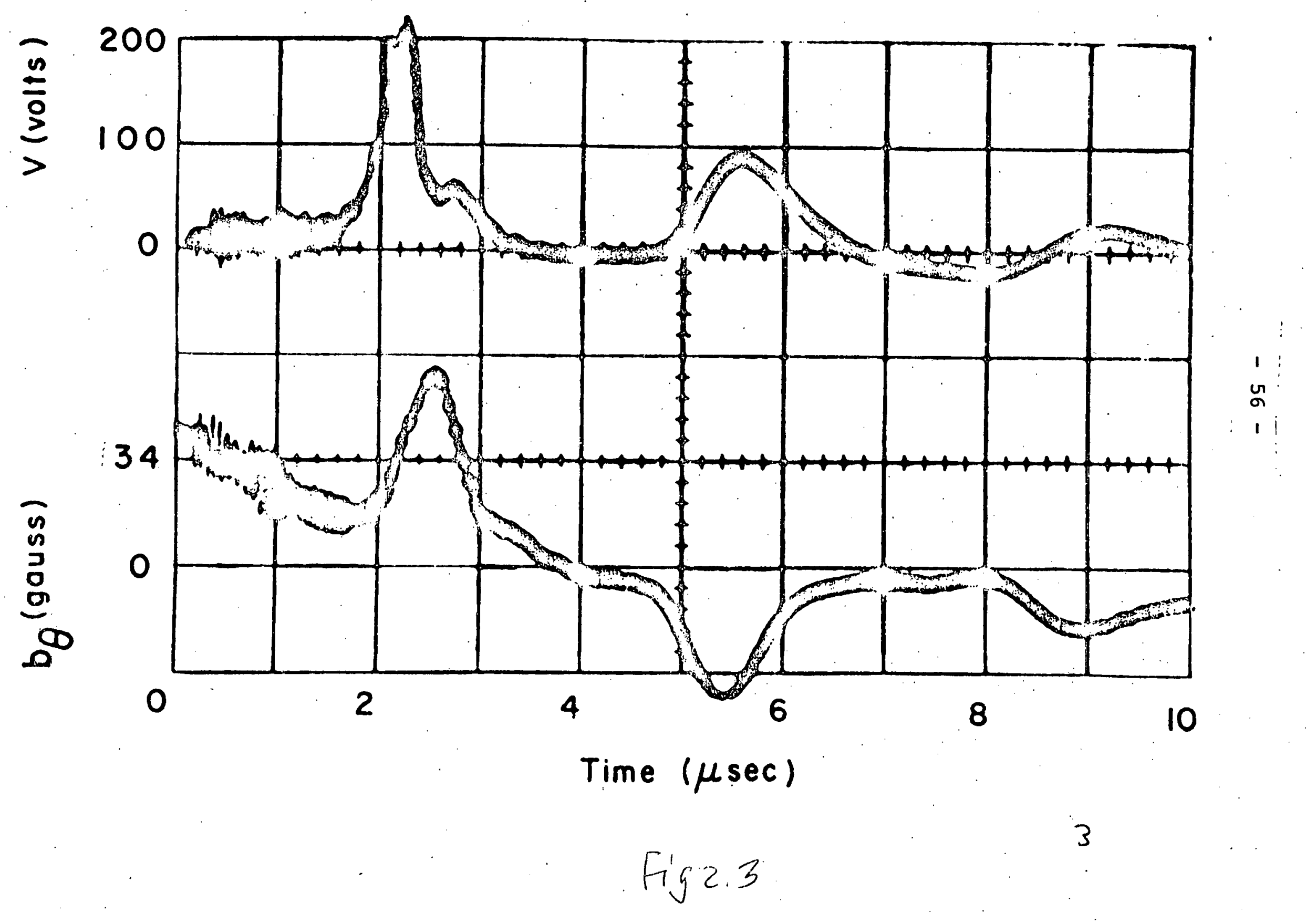




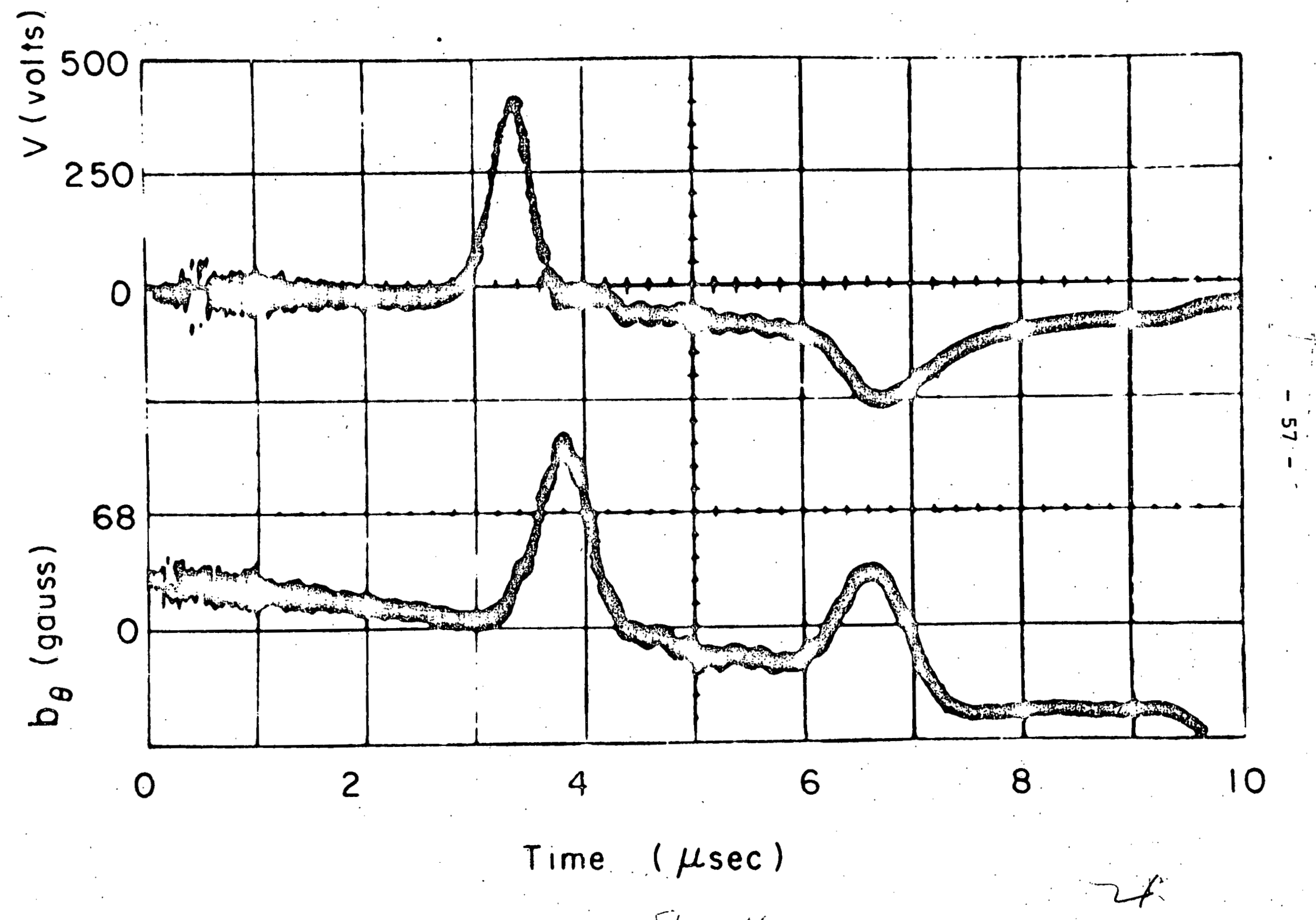




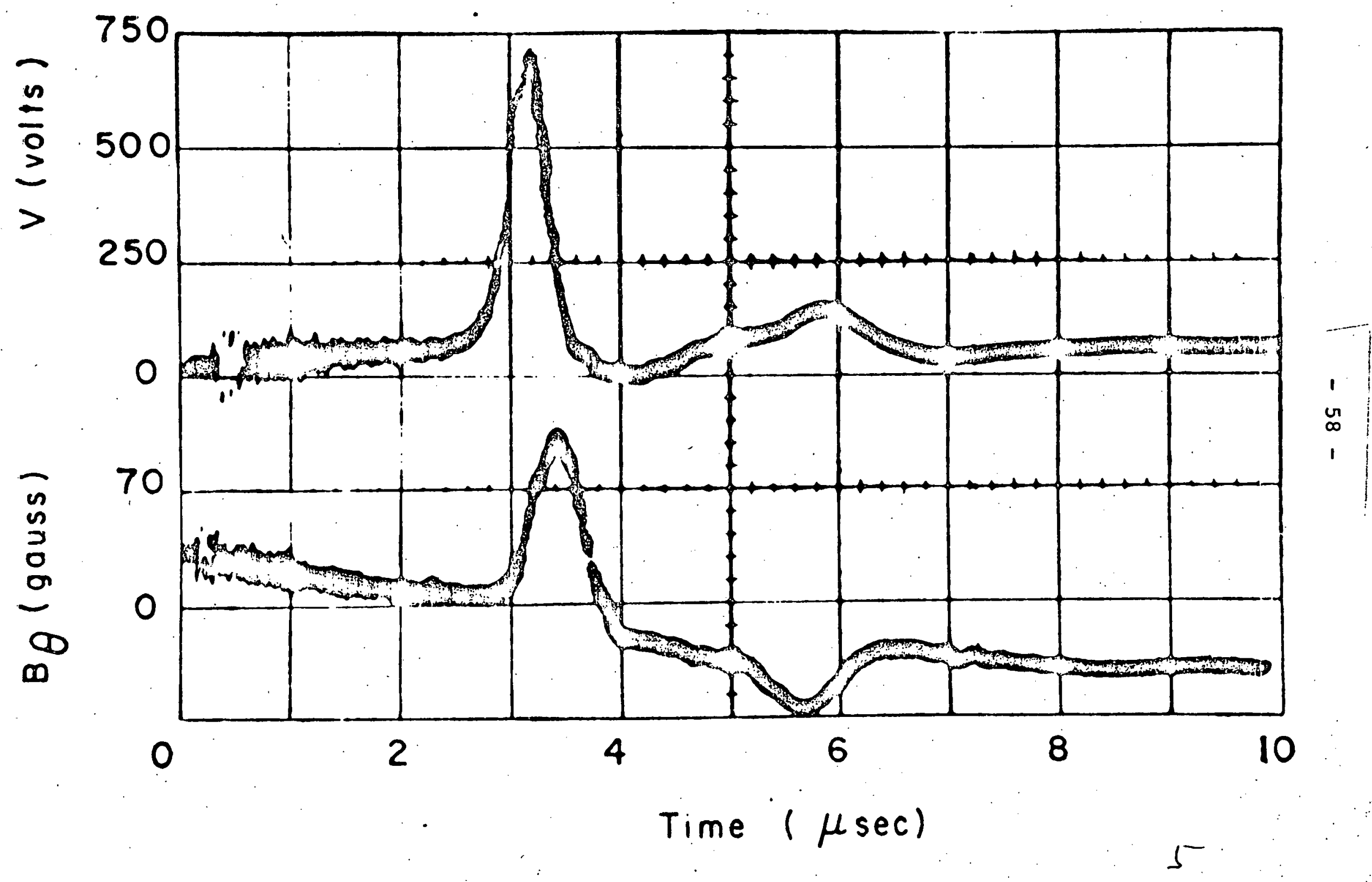

Fig 2.5. 


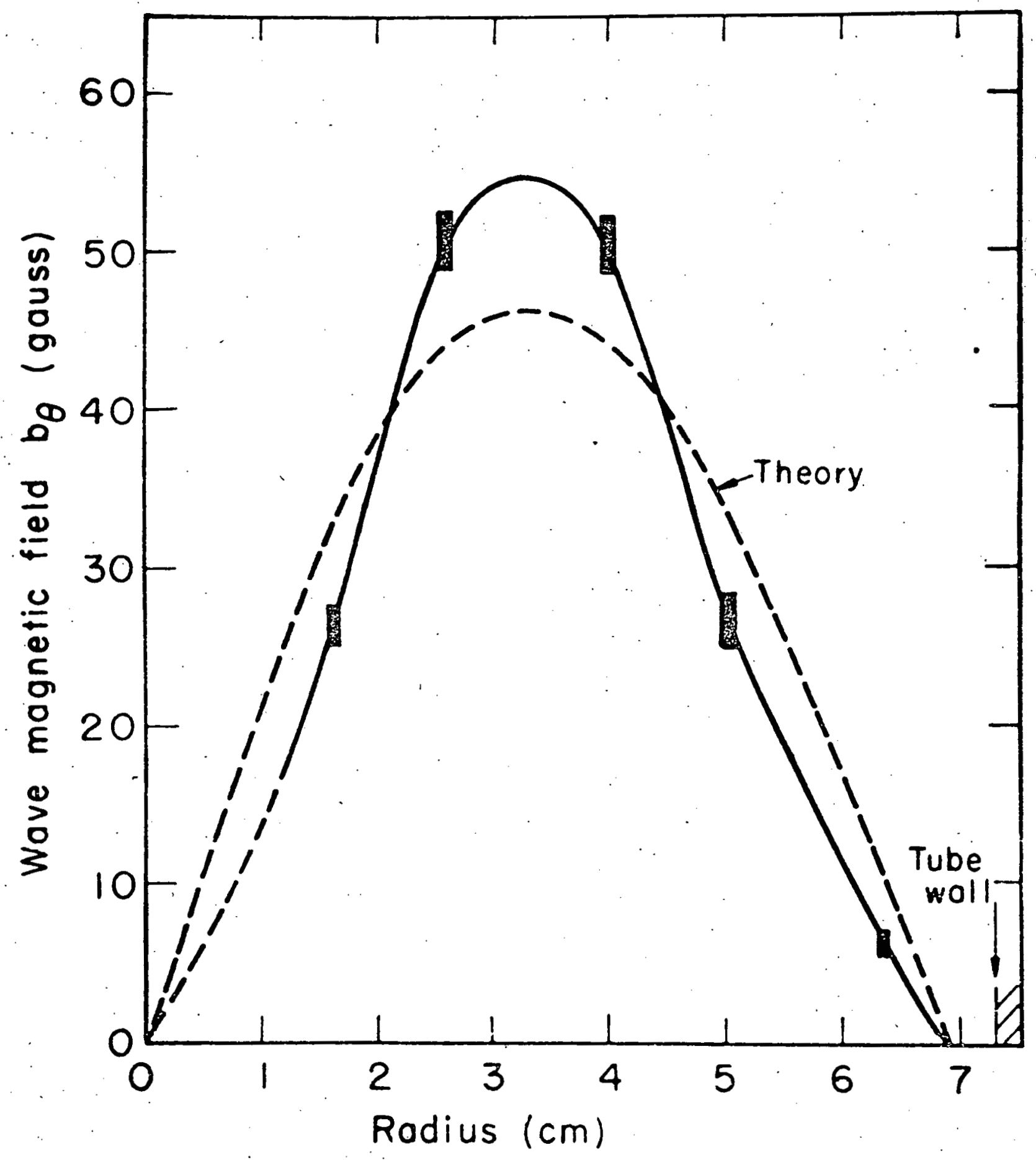

$$
\text { fig } 2.6
$$


insulating boundary, represented by a Pyrex end plate, from a conducting boundary (a copper plate) and from a plasmaneutral interface. The observed change of phase of the wave fields on reflection in Figs. $2.3-2.5$ is seen to be in agreement with the theory.

The magnetic probe measurement of the azimuthal magnetic field $B_{\theta}$ associated. with the wave indicated that the strength of $\mathrm{B}_{\theta}$ was about 100 Gauss. Since the static field is 10,000 Gauss the wave field is about $1 \%$, and therefore a small-amplitude theoretical treatment is valid.

Figure 2.6 gives the radial distribution of ${ }^{B} \theta$ which is described by a first order Bessel function as predicted by the theory.

\subsection{Concluding Remarks}

In low- $\beta$ ideal MHD plasmas the axisymmetric perturbations propagate as two types of decoupled waves; the torsional or shear Alfvén wave and the compressional Alfvén. wave. When pressure perturbations are taken into account the torsional modes are not affected becausc they do not involve any compression of the plasma. The properties of compressional wave however, change considerably and the wave couples to the ion arnustic wave. In a nonuniform plasma, on the other hand the density gradient introduces a frequency dependent coupling between the torsional and compressional waves of uniform plasma. As a result, an axial component of the magnetic field perturbation, which is normally associated 
only with the compressional wave, is now carried by the torsional wave as well, and was in fact detected for low- $\beta$ plasmas at frequencies well below the lowest waveguide cutoff (McPherson and Pridmore-Brown, 1966).. The pressure perturbation is not important for the torsional wave but affects the compressional modes in an uniform plasma. However, in a non-uniform plasma, it plays a very important role. also .. to the torsional mode. This change is rather striking and important for the Alfven wave in nonuniform plasmas; we will discuss it in Chapter IV. 


\section{$\therefore 62-$ \\ REFERENCES FOR CHAPTER II - 1}

Allen, T. K., Baker, W. R., Pyle, R. V., and Wilcox, J. M., Experimental generation of Plasma Alfvén Waves, Phys. Rev. Lett. $-\underline{2}, 383$ (1959).

Brown, I. G., Some experimental observations of the attenuation of Alfvén waves in a laboratory plasma. Aust. J. Phys. 18, 437 (1965).

Herlofson, N., Magnetohydrodynamic waves in a compressible fluid conductor, Nature, 168, 1020 (1950).

Jephcott, D. F., Alfvên waves in a gas discharge, Nature, 183,1652 (1959).

Jephcott, D. F. and Stocker, P. M., Hydromagnetic waves in a cylindrical plasma, J.Fluid, Mec. 13, 587 (1962).

Mc Pherson, D. A. amd Pridmore-Brown, D. C., Density gradient effects on Alfvén wave propagation in a cylinảrical plasma. Phys. Fluids, 9, 2033 (1966).

Newcomb, W. A., The Hydromagnetic Wave Guide. Magnetohydrodynamics, Ed. Rolf K. M. Landshoff, California: Stanford University Press, 1957.

Rukliadze, A. $A$, and silin, V. P., Kinetic theory of drift dissipative instabilities of a plasma. Sov. Phys. Usp. 11, 659 (1969).

Stéfant, R.J., Alfvén wave damping from fluids gyroradius snmpling to the ion acoustic mode, Phys. Fluids 13, 440 (1970).

Stepanov, K.N., Konetic theory of magnetohydrodynamic waves, Sov. Phys. JETP 34, 892 (1958).

Uberoi, C., Low frequency waves in a rotating magnetoplasma, Phys. Fluids 19, 921 (1976). 
Woods, L.C., Hydromagnetic waves in a cylindrical plasma, J. Fluid Mech. 13, 570 (1962).

Wilcox, J.M., Boley, F.I., and Desilva, A.W., Experimental study of Alfvén wave properties, Phys. Fluids $\underline{3}, 15$ (1960).

Wilcox, J.M., DeSilva, A.W., and Cooper, W.S. III, Experiments on Alfvén wave propagation, Phys. Fluids $\underline{4}$, 1506 (1961). 
Aström, E., Magnetohydrodynamic waves in a plasma, Nature, 165, $1019(1950)$.

Aström, E., On waves in an ionized gas. Ark. Fys. 2, 443 (1961).

Bellyustin, N.S. and Tamoikin, V.V., Diffraction of Alfvén in a magnetoactive plasma. Izv. Vuz Radiofiz 16, 1467-74 (1973) .

Cross, R.C. and Lehane, J.A., Propagation of compressional and torsional Alfvén waves under identical plasma conditions. Nucl. Fusion 7,219 (1967).

Chen, F.F., Introduction to Plasma Physics. Plenum Press (1974) .

Daily, W.D., Alfvén wave refraction by interplanetary inhomogeneities. J. Geophys. Res. 78, 2043-53 (1973). Desilva, W., Wilcox, J.M., Cooper, W.S. III, and Boley, F.I., Experiments on Alfvén wave propagation. Magnetohydrodynamics - Proc. IV Bi nnial Gasdynamics Symposium, p. 141, Ed. Cambel, A.B.; Anaerson, T.P.; and Gilawski, M.M., North University Press, 1962.

Fejer, J.A. and Kan, J.R., A guiding centre Vilasov equation and its application to lfvén waves. J. Plasma Phys. (GB) 3, 331-51 (1969).

Hollweg, J.V., Alfvén wave refraction in high-speed solar winds streams. J. Geophys. Res. 80, 908-16 (1975). 
Knox, S.o., Paolini, F.J., and Kristiansen, M., Helical antenna for exciting azimuthally asymmetric Alfvén waves. J. Appl. Phys. 46, 2516-20 (19.75).

Kotik, D.S., Refraction and reflection of an Alfvén wave at a plane boundary with allowance for its transformation into a magnetoacoustic wave. Geomagn and Aeron 10, 779-82 (1970).

Lighthill, M.J., Studies on magnetohydrodynamic waves and other anisotropic wave motions. Phil. Trans. Roy. Soc. (London) 252A, 397 (1960).

Muller, G., Experimental study of torsional Alfvén waves in a cylindrical partially ionized magnetoplasma. Plasma Phys. (GB) 16, 813-22 (1974).

Murphy, G.L., Relativistic effects in magnetohydrodynamic wave propagation. Phys. Fluids 18, 112-14 (1975).

Paoloni, F.J., Boundary effects on $M=0,+O R-1$ Alfvén waves in a cylindrical, collisionless plasma. Phys. Fiuids 18, 640-4 (1975).

Paoloni, F.J., Non-axisymmetric Alfvên wave propagation in an argon plasma. Plasma phys. (GB) 15, 475-81 (1973). Rauchle, E. and Schuller, P.G., Reflection phenomena of torsional Alfvén waves in inhomogeneous magnetic fields. Z. Naturforsch. A 30A, 1594-9 (1975).

Sobolev, S.V., Influence of pressure anisotropy of the nearsolar plasma on the propagation of Alfvén waves. Astron. 2h. 52, 346-50 (1975). 


\section{$\underline{O R-I I-3}$}

Spitzer, L., Physics of fully ionized gases, New York: Interscience Publishers, Inc., 1956.

Stix, T. H., The theory of plasma waves, New York: McGrawHill Book Co., 1962.

Uberoi, C. and Devanathan, C., Small amplitude hydromagnetic waves in compressible medium with Hall Current, Proc.. Ind. Acad. Sci. LX, 42 (1964).

Venkataraman, N. S. and Gustafson, W. A., Alfvén waves associated with long cylindrical satellites, J. Astronaut. Sci. 21, 99-117 (1973). 
III. SPECTRAL ANALYSIS OF IDEAL

ALFVEN WAVE IN INHOMOGENEOUS

MAGNETIC FIELDS

\subsection{Introduction}

In Chapters I and II we have discussed the propagation and properties of the ideal Alfvén wave in the direction of a constant uniform magnetic field. In many cases of interest, however, the magnetic field though constant in time, is not uniform and the magnetic lines of force are, in general, not straight. lines. The question then naturally arises as to what type of solution the hydromagnetic wave equation will admit. Will it have well-defined discrete modes with a dispersion relation which gives rise to wave propagation analogous to the Alfvén wave? These questions will be discussed in this chapter for a class of magnetic fields for which the strength of the magnetic field varies along the direction perpendicular to the plane in which the field lines lie. The choice of this class of magnetic fields derives from the fact that a theoretical study of propagation of Alfvén waves in such fields has led to very striking and important results; the lccal variation of Alfvén wave velocity gives rise to a singularity in the wave equation at the point where the wave phase velocity equals the local Alfvén velocity, which, in turn leads to a continuum spectrum of Alfvén wave frequencies. 
Such a study was initiated by Gajewski and Winterberg (1961). Actually, the equation arrived at by $\mathrm{GW}$ did not show any singular behavior because these authors neglected the effect of pressure perturbations. The basic characteristic of the ideal Alfven waves, as noted in the first chapter, is that the total pressure in the fluid defined as the sum of the fluid and magnetic pressures, remains constant during the passage of the wave as a consequence of the incompressibility condition. But, in the case of inhomogeneous medium the total pressure, in general, couples with the dynamics of the motion and the assumption of neglect of pressure perturbations becomes invalid. Uberoi (1964) considered the problem including the pressure perturbations and found that a singularity arises in the wave equation. She showed that there was no possibility of any dispersion relation. Pridmore-Brown (1966) discussed various features of the singular behavior of the wave equation but did not arrive at the continuum spectrum of Alfvên wave frequencies. It is interesting to note here that Grad in his popular review (1969) mentioned the possibility of existence of a continuum spectrum of Alfvern waves. Uberoi (1972) noted that the Alfvén wave equation exhibits rather complete similarity to that governing high frequency electrostatic oscillations on a cold plasma of nonuniform density. The latter equation had been rigorously analyzed by Barston (1964). This similarity 
made it possible to exploit and to extend the generality, rigor and inclusiveness of Barston's treatment to the case of Alfuén waves.

It is of interest to mention here (Uberoi, 1974) that the wave equation obtained in the study of the following four problems associated with inhomogeneous flows of liquids and plasmas: the two-dimensional shear parallel flow of inviscid incompressible fluid (the Rayleigh problem), the two-dimensional motions of low-density crossed field electron beams, the electrostatic oscillations of inhomogeneous plasma, and Alfvén waves propagation in inhomogeneous magnetic fields show complete similarity to each other. The similarity between the first two problems was pointed out by Briggs, Daugherty and Levy (1970). We note, however, that this singular behavior of the Alfvern wave originates from the assumption that exists behind the ideal magnetohydrodynamics, for example, the zero Larmour radius and/or zero dissipation approximations. It will be shown later that the singularity can be eliminated by relaxing these approximations.

\subsection{The Wave Equation}

The variation in the static magnetic field is assumed to be perpendicular to the plane in which the field iines lie, so that

$$
\underset{\sim 0}{B}(x)=B_{0 y}(x) \cdot \hat{y}+B_{O z}(x) \hat{z}
$$


This is consistent with the initial equilibrium state of the system

$$
\mathrm{v}_{0}=0, \rho_{0}=\text { constant, } \mathrm{p}_{0}+\frac{\left|\mathrm{B}_{0}\right|^{2}}{2 \mu_{0}}=\text { constant. }
$$

We have considered the density to be uniform for the sole purpose to understand in a simple manner the role of varying magnetic field strength on the frequency spectrum associated with Alfvén mode of propagation. It will be. shown in Chapter IV that for incompressible perturbations, the wave equation is similar either when only the magnetic field variation or both the density and magnetic field variations in the direction perpendicular to the magnetic field are considered.

For the initial magnetic field configuration given by Eq. (3.1), the ideal MHD equations for incompressible fluid after linearization, combine to give

$$
\frac{\partial^{2} \underset{v}{v}}{\partial t^{2}}=\frac{1}{\mu_{0} \rho_{0}}(\underset{\sim}{B} \nabla)^{2} \underset{\sim}{v}-\frac{1}{\rho_{0}} \nabla \frac{\partial}{\partial t}\left(p+\frac{\stackrel{B}{0}_{0} \stackrel{B}{\sim}}{\mu_{0}}\right) .
$$

We see from this equation that the total pressure $\tilde{p} \equiv\left(p+\frac{\stackrel{B}{\sim}_{0} \stackrel{B}{\sim}}{\mu_{0}}\right)$ is in general coupled into the dynamics of the motion unless the medium is homogeneous. 
If the medium is homogeneous, we can take the divergence of Eq. (3.2) and obtain $\nabla^{2}\left(\frac{\partial \tilde{p}}{\partial t}\right)=0$, whose only solution vanishing outside a bounded region is $\frac{\partial p}{\partial t}=$ constant, which makes $\tilde{p}=$ constant. This then gives the ordinary Alfvén wave satisfying the equation

$$
\underline{\sim} \underset{v}{v}=0,
$$

where the local Alfvén waveoperator $D$ is given by

$$
D=\mu_{0} \rho_{0} \frac{\partial^{2}}{\partial t^{2}}-\left({\underset{\sim}{B}}_{0} \cdot \nabla\right)^{2}
$$

In the present case $\tilde{p}$ is not constant. We take the $x$ component of the curl of Eq. (3.2),

$$
D(\nabla \times \underset{\sim}{v})=0,
$$

where $(\nabla \times v)_{x}=\left(\frac{\partial v_{z}}{\partial y}-\frac{\partial v_{y}}{\partial z}\right)$.

$$
\text { Eliminating } v_{y^{\prime}} \cdot v_{z} \text { and } \tilde{p} \text { from Eq. (3.2) and }
$$

$\nabla \cdot \underset{\sim}{v}=0$, the final equation, satisfied by $v_{x^{\prime}}$ the velocity component along the direction in which the magnetic field varies, can be put in the form

$$
\nabla \cdot\left[\left(\frac{\partial^{2}}{\partial t^{2}}-\frac{1}{\mu_{0} \rho_{0}}\left(\mathrm{~B}_{0} \cdot \nabla\right)^{2}\right) \nabla v_{x}\right]=0
$$


This equation is to be solved subject to the boundary condition that $v_{x} \rightarrow 0$ as $|x|+\infty$.

Equations (3.4) and (3.5) govern the two modes that can propagate in the inhomogeneous fluid. In general, the individual velocity components are coupled together by the incompressibility condition, $\nabla \cdot \underset{\sim}{v}=0$. In one particular case, there is no coupling, namely, when there is no dependence on $z$. Then $v_{z}$ satisfies $D v_{z}=0$, i.e., it propagates at local Alfvén speed and is uncoupled to $v_{x^{\circ}}$ An even simpler case is the two-dimensional one when the motion is confined to the $x-y$ plane. Then Eq. (3.4) drops out altogether and $v_{z}$ and $v_{y}$ are conveniently computed from a single stream function satisfying Eq. (3.5). In general, however, in any arbitrary velocity disturbance there will be a part, namely, the component of the vorticity, which will propagate one-dimensionally along the field lines at the local Alfvén speed. Other components of the disturbance are, coupled to $v_{x}$ which satisfies the more complicated threedimensional Eq. (3.5). Thus we may expect that at sufficiently large distances, the latter components will die out and only $(\nabla \times v)_{x}$ will survive.

Now, we turn to more detailed analysis of Eq. (3.5). For the Fourier analysis of Eq. (3.5), we take the time and space dependence as $\exp \left[i\left(-\omega t+k_{y} y+k_{z} z\right)\right]$. The equation satisfied by $U_{k \omega}\left(x, k_{y}, k_{z}, \omega\right)$, the Fourier transform of $v_{x}$ is found to be 


$$
\frac{d}{d x}\left(\left[\omega^{2}-\omega_{A}^{2}(x)\right] \frac{d U_{k \omega}}{d x}\right)-k^{2} U_{k \omega}\left[\omega^{2}-\omega_{A}^{2}(x)\right]=0,
$$

where, $\mathrm{k}^{2}=\mathrm{k}_{\mathrm{y}}^{2}+\mathrm{k}_{\mathrm{z}}^{2}, \dot{\omega}_{\mathrm{A}}^{2}(\mathrm{x})=[\underset{\sim}{\mathrm{B}}(\mathrm{x}) \cdot \underset{\sim}{\mathrm{k}}]^{2} / \mu_{0} \rho_{0} \equiv v_{\mathrm{A}}^{2}(\mathrm{x}) \mathrm{k}^{2} \cos ^{2} \theta$ $v_{A}(x)=\left|\sim_{\sim}{ }_{0}\right| /\left(\mu_{0} \rho_{0}\right)^{1 / 2}$, the local Alfvén speed and $\theta(x)$ is the angle between the direction of magnetic field and wave normal direction. Equation (3.6) may be written as:

$$
\frac{d}{d x}\left(E(x) \frac{d U_{k \omega}}{d x}\right)-g(x) f(x) U_{k \omega}(x)=0,
$$

where $f(x)=\ddot{\omega}_{A}^{2}(x)-. \ddot{\omega}^{2},-\infty<x<\infty$

$$
g(x)=k^{2},
$$

the boundary condition being

$$
U_{k \omega}(x) \rightarrow 0 \text { as }|x| \rightarrow \infty \text {. }
$$

\subsection{Solutinn of the Wave Equation}

We consider the magnetic field $\underset{\sim}{\mathrm{B}_{0}}(x)$ to be every where continuous and constant for all sufficiently large $|\mathbf{x}|$ so that the contributions to the discrete spectrum of the regions of constant $\underset{\sim}{\mathrm{B}} 0$ are taken into account. Therefore, the functions $\omega_{A}^{2}(x)$ in the Eq. (3.7) can be considered as real valued, continuous, nowhere constant in some finite interval I and constant outside this interval. Now, we 
shall prove that the necessary condition for the Eq. (3.7) to have nontrivial solutions in this interval satisfying boundary conditions $(3.8)$ is that $\omega^{2}$ must be real and satisfy the inequality. $\quad \min \omega_{A}^{2}(x) \leq \omega^{2} \leq \max \omega_{A}^{2}(x)$. This then defines the continuous spectrum $S=\left\{\omega\left|\min \omega_{A}^{2} \leq \omega^{2} \leq \max \omega_{A}^{2}(x)\right|\right\}$. of the problem.

We assume that $f(x)$ does not vanish anywhere. Multiplying the Eq. (3.7) with $\mathrm{U}_{\mathrm{k}}^{*}$ and integrating from $-\infty$ to $+\infty$ with the boundary conditions, we get

$$
\int_{-\infty}^{\infty} f(x)\left[\left|\frac{d U_{k(1)}}{d x}\right|^{2}+k^{2}\left|U_{k \omega}\right|^{2}\right] d x=0 .
$$

If $\omega$ is complex, such that $\omega^{2} \equiv \omega_{R}^{2}+i \omega_{I}^{2}$, the real and inaginary part of Eq. (3.9) gives

$$
\int_{-\infty}^{\infty} f_{R}(x)\left[\left|\frac{d U}{d x}\right|^{2}+k^{2}\left|u_{k \omega}\right|^{2}\right] d x=0
$$

and

$$
\int_{-\infty}^{\infty} f_{I}(x)\left[\left|\frac{d U_{k \omega}}{d x}\right|^{2}+k^{2}\left|U_{k \omega}\right|^{2}\right] d x=0
$$

From (3.10) and (3.11) we can say that if $f_{I}(x) \neq 0$ and $f_{R}(x) \neq 0$ then $\phi(x) \equiv 0$ is the only solution. Therefore, for a nontrivial $\phi(x), f_{I}(x)=0, i . e ., \omega^{2}$ should be real and $f_{R}(x)=0$, i.e., $\omega^{2}$ should lie in the spectrum $s$. This implies 
that there exists a point $x_{0}$ in the interval $I$ such that $\omega^{2}=\omega_{A}^{2}\left(x_{0}\right)$.

Returning to Eq. (3.7), the points $x=x_{0}$ for which $f\left(x_{0}\right)=0$, i.e., $\left|v_{A}(x) \cos \theta(x)\right|=|\omega / k|$ are the singular points of the Eq. (3.7) and so the eigenfunctions $U_{k \omega}(x)$ may not be well behaved there. We shall now investigate the behavior of the solutions of this equation near such a singular point.

Let $f(x)$ and $g(x)$ be analytic functions in a neighborhood of $\mathrm{x}_{0}$, such that,

$$
f(x)=\sum_{n=1}^{\infty} f_{n}\left(x-x_{0}\right)^{n},
$$

where $f_{n}(x) \neq 0$ for $n \geq 1$. Then near $x_{0}$, which is a regular singularity, the general series solution of the Eq. (3.7) is of the form

$$
v_{k \omega}(x)= \begin{cases}A_{1} W(x)+B_{1}\left[W(x) \ln \left|x-x_{0}\right|+\left(x-x_{0}\right) V(x)\right], & x>x_{0} \\ A_{2} W(x)+B_{2}\left[W(x) \ln \left|x-x_{0}\right|+\left(x-x_{0}\right) V(x)\right], & x<x_{0},\end{cases}
$$

where $A_{1}, A_{2}, B_{1}, B_{2}$ are constants $W(x), V(x)$ are analytical in a neighborhood of $x_{0}$, and $w\left(x_{0}\right)=1$. Thus the general solution of Eq. (3.7) diverge at the point $x=x_{0}$, the divergence being logarithmic.

It is easily shown that these singularities will appear in solutions which obey the boundary conditions (3.8). 
Hence, in general the mode solutions of (3.7) are singular. But, a well-behaved solution of the original boundary value problem can be constructed in the realm of generalized functions by making an integral superposition over the whole spectrum $\mathbf{S}$ of these eigenfunctions with some expansion coefficient $A_{k}(\omega)$ such that

$$
U_{k}(x, t)=\int_{S} A_{k}(\omega) U_{k \omega}(x) e^{-i \omega t} d \omega
$$

We shall now try to determine the arbitrary constants $A_{1}, A_{2}, B_{1}, B_{2}$ by applying certain matching relations which must be satisfied across the singular point $x=x_{0}$. We prove that $A_{1}$ and $A_{2}$ are arbitrary, while $B_{1}=B_{2}$. To prove this we define,

$$
\begin{aligned}
& \mathrm{U}_{1} \equiv \mathrm{W}(\mathrm{x}) \mathrm{H}\left(\mathrm{x}-\mathrm{x}_{0}\right) \\
& \mathrm{U}_{2} \equiv \mathrm{B}_{1}\left[\mathrm{~W}(\mathrm{x}) \ln \left|\mathrm{x}-\mathrm{x}_{0}\right|+\left(\mathrm{x}-\mathrm{x}_{0}\right) \mathrm{V}(\mathrm{x})\right] \\
& \mathrm{U}_{3} \equiv \mathrm{B}_{2}\left[\mathrm{~W}(\mathrm{x}) \ln \left|\mathrm{x}-\mathrm{x}_{0}\right|+\left(\mathrm{x}-\mathrm{x}_{0}\right) \mathrm{V}(\mathrm{x})\right] \\
& \mathrm{U}_{4} \equiv \mathrm{U}_{2} \mathrm{H}\left(\mathrm{x}-\mathrm{x}_{0}\right)+\mathrm{U}_{3} \mathrm{H}\left(\mathrm{x}_{0}-\mathrm{x}\right)
\end{aligned}
$$

where $H(x)$ is the Heaviside function. Then we have from (3.12)

$$
U_{k, j}(x)=A_{2} W(x)+\left(A_{1}-A_{2}\right) U_{1}+U_{4} \cdot
$$


It is then sufficient to show that.

$$
\frac{d}{d x}\left[f \frac{d U_{1}}{d x}\right]-g(x) f(x) U_{1}=0,
$$

and

$$
\frac{d}{d x}\left[f \frac{d U_{4}}{d x}\right]-g(x) f(x) U_{4}=\left(B_{1}-B_{2}\right) \frac{d f\left(x_{0}\right)}{d x} \delta\left(x-x_{0}\right)
$$

Note that $f(x)=\sum_{n=1}^{\infty} f_{n}\left(x-x_{0}\right)^{n}, f_{1} \neq 0, f\left(x_{0}\right)=0, W\left(x_{0}\right)=1$; $\mathrm{dH}\left(\mathrm{x}-\mathrm{x}_{0}\right) / \mathrm{dx}=\delta\left(\mathrm{x}-\mathrm{x}_{0}\right)$, and that $\mathrm{y}(\mathrm{x}) \delta\left(\mathrm{x}-\mathrm{x}_{0}\right)=\mathrm{y}\left(\mathrm{x}_{0}\right) \delta\left(\mathrm{x}-\mathrm{x}_{0}\right)$ if $y(x)$ is continuous at $x_{0}$. We shall first prove that $A_{1}$ and $A_{2}$ are arbitrary.

We have

$$
\frac{d U_{1}}{d x}=\frac{d W}{d x} H\left(x-x_{0}\right)+\delta\left(x-x_{0}\right)
$$

and so we find

$$
f \frac{d U}{d x}=f \frac{d W}{d x} H\left(x-x_{0}\right)
$$

Therefore

$$
\begin{aligned}
\frac{d}{d x}\left[f \frac{d U_{1}}{d x}\right] & =H\left(x-x_{0}\right) \frac{d}{d x}\left[f \frac{d W}{d x}\right]+f \frac{d W}{d x} \delta\left(x-x_{0}\right) \\
& =H\left(x-x_{0}\right) \frac{d}{d x}\left[f \frac{d W}{d x}\right],
\end{aligned}
$$


and so we obtain

$$
\frac{d}{d x}\left[f \frac{d U_{1}}{d x}\right]-g \text { f } U_{1}=H\left(x-x_{0}\right)\left[\frac{d}{d x}\left(f \frac{d w}{d x}\right)-g f w\right]=0
$$

Therefore, it is demonstrated that $A_{1}$ and $A_{2}$ are arbitrary. Let us now show that $B_{1}=B_{2}$. For this we define a function $G(x)$ as follows :

$$
G(x)=\left\{\begin{array}{cc}
f(x) \frac{d U_{2}}{d x} & x>x_{0} \\
f(x) \frac{d U_{3}}{d x} & x<x_{0}
\end{array}\right.
$$

For very small values of $\varepsilon, \varepsilon>0$, we have

$$
\begin{aligned}
& \frac{d U_{2}\left(x_{0}+\varepsilon\right)}{d x}=B_{1}\left[\frac{d W\left(x_{0}+\varepsilon\right)}{d x} \ln +\frac{W\left(x_{0}+\varepsilon\right)}{\varepsilon}+\varepsilon \frac{d V\left(x_{0}+\varepsilon\right)}{d x}+V\left(x_{0}+\varepsilon\right)\right] \\
& \frac{d U_{3}(x-\varepsilon)}{d x}=B_{2}\left[\frac{d W(x-\varepsilon)}{d x} \ln \varepsilon-\frac{W\left(x_{0}-\varepsilon\right)}{\varepsilon}-\varepsilon \frac{d V(x-\varepsilon)}{d x}+v\left(x_{0}-\varepsilon\right)\right],
\end{aligned}
$$

since

$$
f(x)=\sum_{n=1}^{\infty} f_{n}\left(x-x_{0}\right)^{n}, \quad f_{1}=\frac{d f\left(x_{0}\right)}{d x} \neq 0,
$$

we find

$$
G\left(x_{0}+\right)=\lim _{\varepsilon \rightarrow 0} f\left(x_{0}+\varepsilon\right) \frac{d U_{2}\left(x_{0}+\varepsilon\right)}{d x}=B_{1} f_{1} W\left(x_{0}\right)=B_{1} f_{1} \text {, }
$$




$$
\left.G\left(x_{0}\right)^{-}\right)=\lim _{\varepsilon \rightarrow 0} f\left(x_{0}+\varepsilon\right) \cdot \frac{\mathrm{dU}_{3}\left(x_{0}+\varepsilon\right)}{d x}=B_{2} f_{1}
$$

and so $G\left(x_{0}+\right)-G\left(x_{0}-\right)=\left(B_{1}-B_{2}\right) \frac{d f\left(x_{0}\right)}{d x}$, which shows that $B_{1}=B_{2}$.

The fact that the coefficients $A_{1}$ and $A_{2}$ are independent across a singularity precludes any functional dependence of $w$ on $k$, i.e., given any fixed value of $k, \omega$ is free to range over the continuous spectrum $S$ and so our medium does not give rise to dispersion relations. Wellbehaved solutions to Eq. (3.5) can be obtained by integrating their singular modes over the spectrum $S$. The dominant terms of the asymptotic expansion for $U_{k}(x, t)$ as estimated by Barston (1964) are

$$
\mathrm{u}_{\mathrm{k}}(\mathrm{x}, \mathrm{t}) \sim \frac{1}{t} \mathrm{e}^{ \pm \omega_{\mathrm{A}}(\mathrm{x})}+0\left(1 / \mathrm{t}^{2}\right) .
$$

Thus, each infinitely thin fluid layer oscillated with the Alfven wave frequency $\omega_{A}(x)$ and the oscillations are damped as the inverse of time.

Hence, if $\omega_{A}(x)$ is continuous and nowhere constant in an interval $I$, the MHD plasma admits of only singular modes and an entirely continuous spectrum. No dispersion relation exists. The spectrum consists of those values of $\omega$ that satisfy the equation $\omega^{2}=\omega_{A}^{2}(x)$ for some $x \in I$. We 


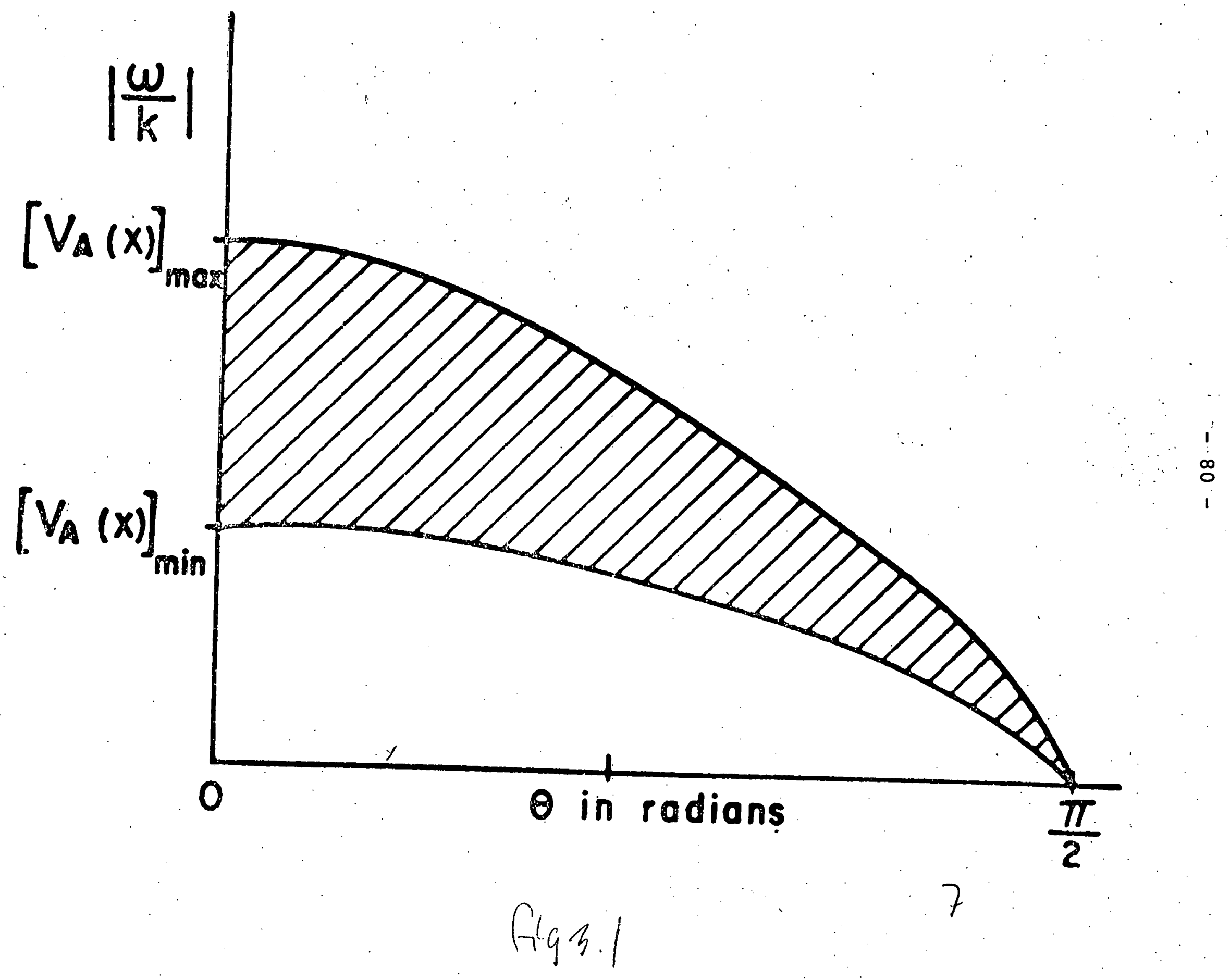


note that the anisotropy induced by the magnetic field due to which the bandwidth $\left[\min \omega_{\dot{A}}^{2}(x) \cos ^{2} \theta(x) \leq \omega^{2} \leq\right.$ $\left.\max \omega_{A}^{2}(x) \cos ^{2} \theta(x)\right]$ of the continuous spectrum continuously changes with $\theta$, cannot be considered as a dispersion in the classical sense (see Fig. 3.1).

What about the discrete spectrum? suppose $\omega_{\mathrm{A}}^{2}(\mathrm{x})$ assumes constant values $\omega_{\mathrm{A} 1}^{2}$ and $\omega_{\mathrm{A} 2}^{2}$ on either side of the open interval $I$, then $\pm \omega_{A 1}$ and $\pm_{A 2}$ will form the entire discrete spectrum of the system. But, if jump discontinuities are introduced in the function $\omega_{A}(x)$ in the interval $I$, such that $\omega_{A}(x)$ jumps discontinuously from a value $\omega_{A i}$ to a value $\omega_{A i+1}\left(>\omega_{A i}\right)$ upon crossing a surface $s_{i}$ [i.e., planes. parallel to $(y, z)$ planel and if $\left(\omega_{A i}, \omega_{A i+1}\right)$ and the range of $\omega_{A}(x)$ have no point in common, then we can get well behaved modes, the number being equal to the number if discontinuities, which will also form part of the discrete spectrum. The well behaved modes arising due to step-discontinuity in the function $\omega_{A}(x)$ are the well known Alfven surface modes, the existence and properties of these will be discussed in Chapter IV.

\subsection{Concluding Remarks}

Knowledge of the existence of a continuum spectrum for Alfvén waves plays an important role in practical problems both in space plasmas where inhomogeneity is both due to the density variation and structured magnetic fields and in laboratory plasmas where the density is nonuniform. 
In space plasmas this knowledge has led to an understanding of the phenomenon of magnetic pulsations in the ULF ranges (Lanzerotti et al., 1973; Chen and Hasegawa, 1974).

A practical method for heating plasmas in fusion experiments by creating conditions for spatial resonance of the Alfvén wave has been proposed. This aspect will, be discussed in detail in Chapter VII.

The understanding of the spectral theory of magnetohydrodynamics has led to a new approach in the study of MHD stability theory. Relevant early references are Grad (1973); Goedbloed and Sakanaka (1974) and Goedbloed (1975)

The inhomogeneous nature of solar magnetic fields has been firmly established by observations (e.g., Harvey, 1971). We believe that the theory of Alfvén waves in structured magnetic fields presented in this chapter will play an important role in delimiting various effects in solar physics. 


\section{REFERENCES FOR CHAPTER III-I}

Barston E. M., Electrostatic oscillations in inhomogeneous cold plasmas, Annals of Physics, 29, 282 (1964).

Briggs, R. J., Daugherty, J. D., and Levy, R. H., Role of

Landau damping in crossed-field electron beams and inviscid shear flow; Phys. Fluids, 13, 421 (1970).

Chen, L., and Hasegawa, A., A theory of long-period magnetic pulsations: 1. Steady state excitation of field line resonance. J. Geophys. Res. 79,1024 (1974).

2. Impulse excitation of surface eigenmode. J. Geophys. Res. 79, 1033 (1974).

Grad, H., Frontiers of Physics Today: Plasma Phys. Today, 22, No. 12,34 (1969).

Grad, H., Magnetofluid-dynamic spectrum and low shear stability, Proc. Nat. Acad. Sci. USA, 70, 3277 (1973).

Goedbloed, J. P. and Sakanaka, P. H., New approach to magnetohydrodynamic stability: a practical stability concept, Phys. Fluids, 16, 908 (1974).

Goedbloed, J. P., Spectrum of ideal magnetohydrodynamics of axially symmetric toroidal systems, Phys. Fluids, 18, 1258 (1975).

Gajewski, R. and Winterberg, F., Alfvén waves in inhomogeneous magnetic fields. Boeing Scientific Research Laboratories Report D1-82-0111 (1961): later published in Annals of Phys. 32, 348 (1965). 
Harvey, J., Publ. Astron. Soc. Pacific 83, 539 (1971).

Lanzerotti, L. J., Fukunishi, H., Hasegawa, A., and Chen, L., Excitation of the plasmapause at ultra low frequencies, Phys. Rev. Letts. 31, 624 (1973).

Pridmore-Brown, D. C., Alfvén in a stratified incompressible fluid. Phys. Fluids, 9, 1220 (1966).

Uberoi, C., Hydromagnetic waves in ideally conducting uniform medium with inhomogeneous magnetic field of constant direction. Ind. J. Pure and Appl. Phys. 2, 133 (1964). Uberoi, C., Alfvén waves in inhomogeneous magnetic fields. Phys. Fluids, 15, 1673 (1972).

Uberoi, C., On the similarities detected in waves in plasmas and waves in incompressible fluids. Applied Mathematics Seminars, Indian Institute of Science, Bangalore (1974). 


\section{OTHER REFERENCES FOR CHAPTER III-1}

De utsch, R.F. and Ralan, L., The propagation of lfven waves in nonhomogeneous systems in relativistic magnetohydrodynamics. Bull. Inst. Politeh. IASI 14 , 175-8 (1968).

Deutsch, R.V. and Fornwald, F., Propagation of 1 fvén waves in non-homogeneous plasma in the presence of the homogeneous gravitational field. Rev. Roumaine Phys. 13, 895-900 (1968).

Goedbloed, J.P., Spectrum of ideal magnetohydrodynamics of axisymmetric toroidal systems. Phys. Fluids 18, 1258-68 (1975).

Hasegawá, A. and Liu Chen, Theory of magnetic pulsations. Space Sci. Rev. 16, 347-59 (1974).

Infeld, E., Solutions of the linearized equations of magnetohydrodynamics in nonhomogeneous magnetic fields. Phys. of Fluids 12, 1845-8 (1969).

Morrow, R. and Brennan, M.H., The propagation of compressional 1 fuén waves in nonuniform plasmas. Aust. J. Phys. 27, 181-94 (1.974).

Muller, G.; Rauchle, R.; and Schuller, P.G., Investigation of plasma-magnetic waves in an inhomogeneous cylindrical plasma. Plasma Phys. (GB) 15, 925-8 (1973).

Swanson, D.G. and Ngan, Y.C., Warm-plasma effects on fastAlfvén-wave cavity resonances. 'Phys. Rev. Lett. 35, 517-20 (1975).

Tataronis, J.A., Energy absorption in the continuous spectrum of ideal MHD. J. Plasma Phys. (GB) 13, 87-105 (1975). 


\section{RESONANT ABSORPTION AND MODE CONVERSION OF ALFVEN SURFACE WAVES IN NONUNIFORM}

\section{PLASMAS}

\subsection{Introduction}

The study of Alfvên wave propagation in nonuniform cylindrical plasmas considered in the second chapter was limited to low- $\beta$ plasmas with the assumption that the pressure perturbation does not hold for high- $\beta$. plasmas. Moreover, as noted in the third chapter the coupling of pressure perturbations to the dynamics of the motion can completely change the properties of Alfvén wave propagation in inhomogeneous media. The Alfvên wave equation shows a singular behavior at the point where the externally applied frequency $\omega$ matches with the local Alfvén (shear Alfvén) frequency $k_{\|}(x) v_{A}(x)$, where $k_{\|}(x)$ is the local wave number parallel to the ambient magnetic field. Due to the presence of this singularity a nonuniform plasma with continuously varying density, is characterized by a continuum spectrum in the hydromagnetic frequency regime. On the other hand, if jump discontinuities are introduced in the density profile, the system admits of discrete spectrum. The incompressible perturbations in the hydromagnetic regime give rise to Alfvên surface waves propagating along the discontinuous plane with definite frequencies. Most of the instabilities which originate from the nonuniformity of a plasma are in fact the instabilities of a surface wave (Hasegawa, 1973). (The instabilities associated with Alfvén surface waves will be discussed in Chapter V.). 
In the earlier part of this chapter, we shall be concerned with the question of how to unite the two dissimilar pictures presented above; that is to say, we ask what happens to the surface wave when the discontinuous density profile is smoothed and whether there are some collective modes of propagation even in the continuous case. We shall, therefore, treat the Alfvén wave equation (similar to the Eq. (3.5) in Chapter III) afresh, by using the Laplace transform technique to solve the corresponding initial-value problem. Once again, using the analog between Alfvén wave equation and the plasma wave equation we follow closely the analysis given by sedlacek (1971) for the study of electrostatic oscillations of cold inhomogeneous plasma. This approach, though equivalent to the normal-mode analysis is known to provide results in a form suitable for finding the whole asymptotic time expansion (not just the dominant terms) of the solution simply by studying the singularities of its Laplace transform in the complex frequency plane. By constructing the Green function of the wave equation we find that there are branch point singularities on the real axis of the complex $\omega-p l a n e$, which correspond to the continuous spectrum, and which, asymptotically, give rise to noncollective oscillations with position-dependent frequency and damping proportional to negative powers of time. In addition, there are an infinite number of new singularities 
(simple poles) of the analytic continuation of the Green function into the lower half of the complex-w plane with position independent frequency so that they represent exponentially damped, collective (surface eigenmodes) modes of wave propagation. Thus, the effect of a continuous density profile is to introduce damping to the originally undamped surface modes of a discontinuous plasma. This damping arises due to the presence of the continuous frequency spectrum. The normal modes which make up the continuum can phase mix in time, leading to decay of macroscopic variables. The damping of Alfvén surface waves is thus similar to the well known Landau damping of plasma oscillations in warm plasma. The damping of surface waves has been observed experimentally (Grossmann et al., 1973) and in a magnetohydrodynamic computer calculation (Pritchett and Dawson, 1978). Another aspect of the absorption of surface waves, is that the system can irreversibly absorb energy when it is externally driven. The energy absorption rate in the presence of an external driving source which is represented by a sheet current located in the vacuum will be discussed in section 4.4 . The absorption is a consequence of the local Alfvén resonance which occurs when the externally applied frequency matches to that in the continuum. A characteristic of this absorption is that it occurs locally in the space in which the continuum is defined. The relationship between a continuous 


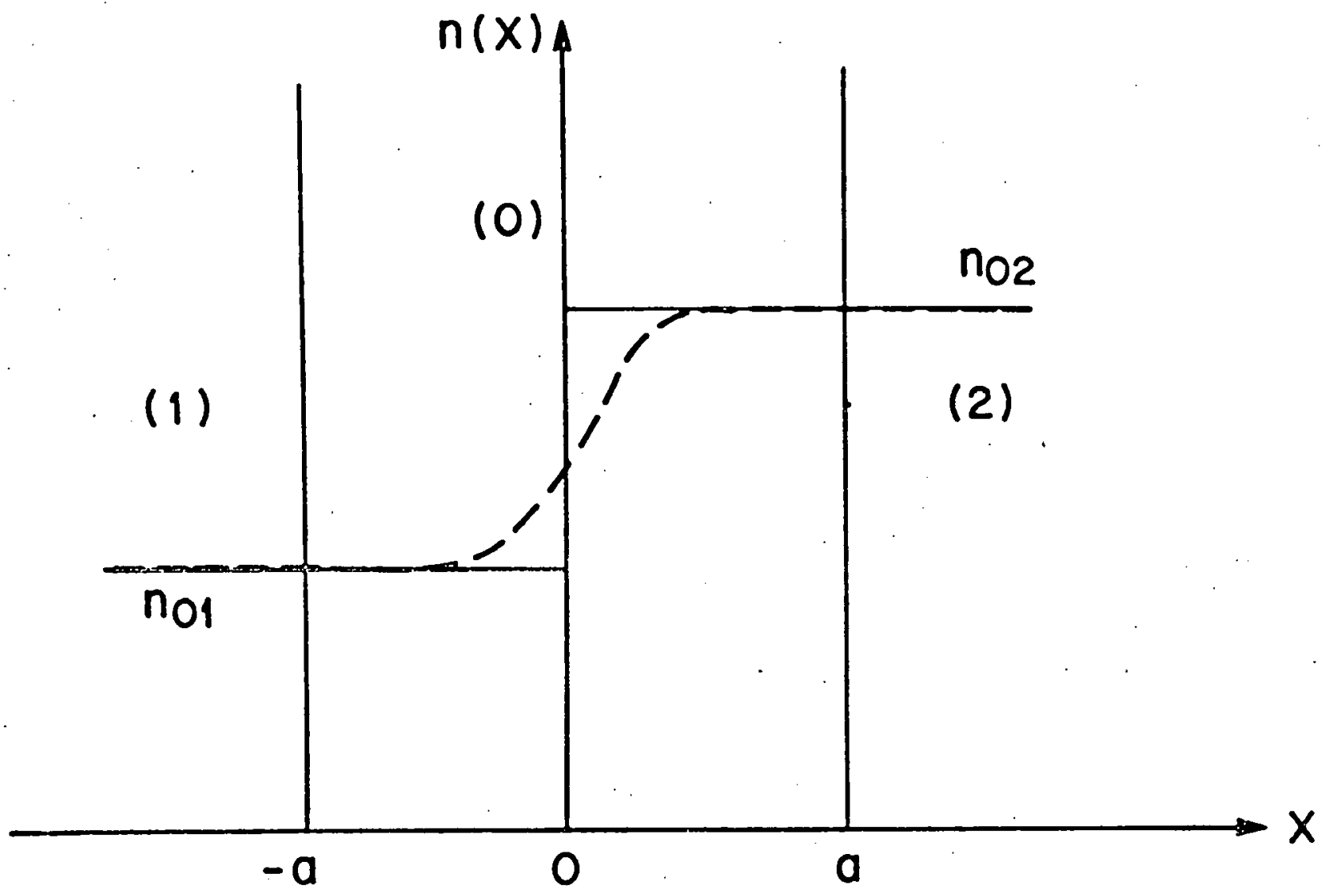

Fig 4.1 
spectrum and energy absorption has been noted and discussed extensively for zero-temperature plasma oscillation in a nonuniform plasma (Baldwịn and Ignat, 1969).

Extensive studies on linear transformation and absorption of electromagnetic waves in nonuniform plasma haverevealed the fact that collisionless resonant absorption of waves in a plasma is connected with wave transformation (review by Golant and Piliya, 1972). In section 4.4, we study the resonant mode conversion of the Alfvén surface waves (Hasegawa and Chen, 1976) which will enable us to. understand the mechanism by which the surface wave energy is absorbed and propagated in the plasma, away from the resonant region.

\subsection{Alfvến Surface Wave}

Let us consider a plasma with a sharp density gradient (cf. Fig. 4.1).

$$
\begin{array}{ll}
n(x)=n_{01} & x \geq 0 \\
n(x)=n_{02} & x<0
\end{array}
$$

We shall also consider discontinuity in the magnetic field, taken along the z-direction

$$
\begin{array}{ll}
{\underset{\sim}{\mathrm{B}}}_{0}=\mathrm{B}_{01} \hat{\mathrm{z}} & \mathrm{x} \geq 0 \\
\underset{\sim}{\mathrm{B}}=\mathrm{B}_{02} \hat{\mathrm{y}} & \mathrm{x}<0 .
\end{array}
$$

Taking the ideal MHD model for the plasma, we consider incompressible perturbations in the $y-z$ plane given by $f(x) e^{i\left(-\omega t+k_{y} y+k_{z} z\right)}$. We seek for the surface wave solutions 


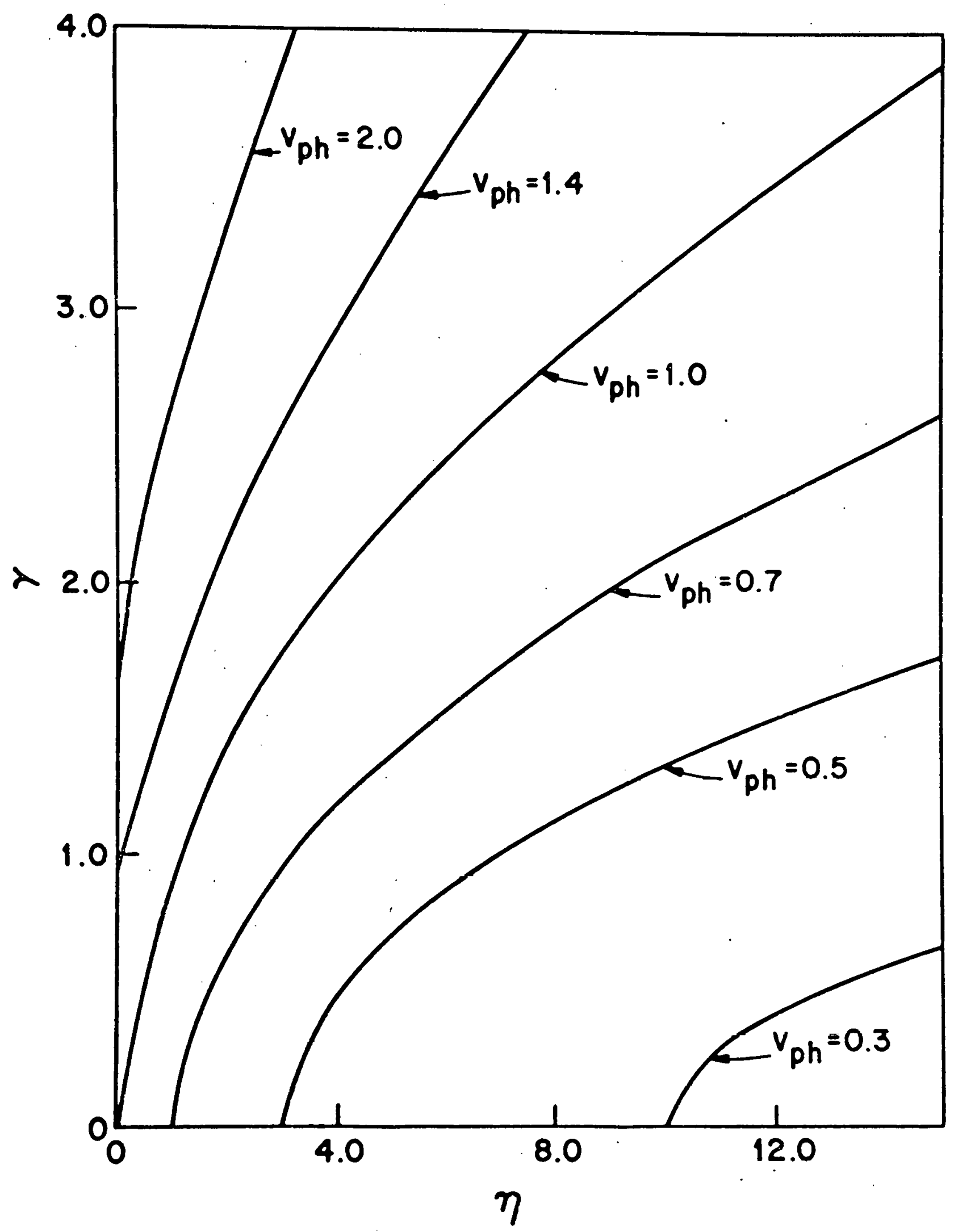

Fig. 4.2 
such that the wave amplitudes decay away from the interface $\mathrm{x}=0$.

Following the procedure in the chapter III, the basic MHD equations after linearization can be written in the form

$$
\begin{aligned}
{\left[\frac{\partial^{2}}{\partial t^{2}}-\frac{\left(\mathbb{\sim}_{0} \cdot \nabla\right)^{2}}{\mu_{0} \rho_{0}}\right] \underset{\sim}{v} } & =-\frac{1}{\rho_{0}} \frac{\partial}{\partial t} \nabla \tilde{p} \\
\nabla \cdot \underset{\sim}{v} & =0,
\end{aligned}
$$

where $\tilde{p}=\left(p+\frac{\stackrel{B}{0}_{0} \cdot B}{\mu_{0}}\right), \rho_{0}=m_{i} n_{0}$ with $m_{i}$ the ion mass and $n_{0}$ the unperturbed plasma density. Eliminating p from (4.1) and $(4.2)$ gives

$$
\nabla^{2} v_{x}=0
$$

to be satisfied both in regions 1 and 2 .

Therefore, the equation governing the surface perturbations is the Laplace equation with the boundary conditions $v_{x}$ and $\tilde{p}$ being continuous. From Eq. (4.1) $\tilde{\mathrm{p}}$ can be written as

$$
\tilde{\mathrm{p}}=\frac{1}{\omega \mathrm{k}^{2}}\left(\omega^{2} \rho_{0}-\frac{\mathrm{B}_{0}^{2} \mathrm{k}^{2} \perp}{\mu_{0}}\right) \frac{\partial \mathrm{v}_{\mathrm{x}}}{\partial \mathrm{x}},
$$

where $k_{\mid l} \equiv k_{z}, k_{1} \equiv k_{y}$ and $k^{2}=k_{\mid}^{2}+k_{1}^{2}$. The boundary conditions therefore are continuity of $v_{x}$ and $\varepsilon \frac{\partial v_{x}}{\partial x}$ with $\varepsilon=\frac{1}{k^{2}}\left(\omega^{2} \rho_{0}-\frac{B_{0}^{2} k^{2} \mid}{\mu_{0}}\right)$. 
The solution of (4.3) for surface waves is given

as

$$
\left.\begin{array}{ll}
v_{x}=A e^{-|k| x} & x>0 \\
v_{X}=B e^{+|k| x} & x<0 .
\end{array}\right\}
$$

Applying the boundary conditions we get the following dispersion relation for the surface waves:

$$
\rho_{01}\left(\frac{\omega^{2}}{k_{||}^{2}}-v_{A 1}^{2}\right)+\rho_{02}\left(\frac{\omega^{2}}{k_{||}^{2}}-v_{A 2}^{2}\right)=0 .
$$

Considering a plasma-vacuum interface, Eq. (4.6) with $\rho_{02}=0$ gives the following dispersion relation for the Alfvén surface waves (Kruskal and Schwarzschild, 1954)

$$
\omega_{A s}=\left(\frac{B_{01}^{2}+B_{02}^{2}}{\mu_{0} \rho_{0}}\right)^{\frac{3}{2}} k_{\mid} .
$$

In the parlicular case w1th $\mathrm{B}_{01}=\mathrm{B}_{02}$, this frequency becomes (Chen and Hasegawa, 1974)

$$
\omega_{\mathrm{As}}=\sqrt{2} \mathrm{v}_{\mathrm{A}} \mathrm{k}_{\|}
$$

We note that the surface wave frequency in this case is higher than the Alfven wave frequency (the bulk resonant frequency) in contrast to the electrostatic surface wave where the surface wave frequency is given by $\omega_{s}=\omega_{p} / \sqrt{2}$. 
In general the Eq. (4.6) will have real roots only when the surface phase velocity satisfies the following inequality:

$$
\mathrm{v}_{\mathrm{A} 1}^{2} \cos ^{2} \theta<\omega^{2} / \mathrm{k}^{2}<\mathrm{v}_{\mathrm{A} 2}^{2} \cos ^{2} \theta
$$

where $\theta$ is the angle between $\underset{\sim}{\mathbf{k}}$ and $\underset{\sim}{\mathrm{B}} 0^{\cdot}$ This condition was obtained in Chapter III from Barston's theorem.

$$
\text { Defining } n=\rho_{02} / \rho_{01} \text { and } \gamma=B_{02} / B_{01} \text { Eq. (4.6) }
$$

can be written as

$$
\mathrm{v}_{\mathrm{ph}}^{2}=\frac{1+\gamma^{2}}{1+\eta},
$$

where $v_{p h} \equiv w /\left(k_{\|} v_{A l}\right)$. Fig. 4.2 gives an estimate of the surface parameters $\eta$ and $\gamma$ for given ratios of surface wave velocity to Alfvén wave velocity $\mathrm{v}_{\mathrm{Al}}$.

\subsection{Resonant Absorption of Surface Waves}

Instead of having a sharp density discontinuity we now consider the situation in which the plasma density is smoothly varying in $x$. For this we consider a small transition layer $-a<x<a$ in which the density varies smoothly. The density profile, as compared to section 2, then looks like as shown by the broken line in Fig. 4.1 . 
To study the surface waves in region $(0)$ we proceed as in Chapter III to obtain the wave equation, with $\underset{\sim}{\mathrm{B}} \mathrm{O}={\underset{\sim}{\mathrm{B}}}_{0} \hat{z}$ to be uniform and density $\rho_{0} \equiv \rho_{0}(\mathrm{x})$ continuously varying with $x$,

$$
\nabla \cdot\left\{\left[\rho_{0}(x) \frac{\partial^{2}}{\partial t^{2}}-\frac{\left(\mathrm{B}_{0} \cdot \nabla\right)^{2}}{\mu_{0}}\right] \nabla v_{x}\right\}=0,
$$

Equation (4.8) shows the coupling of bulk Alfven waves with the surface Alfvén waves. Considering the perturbations independent of the coordinate $y$, the Fourier analysis with respect to $\mathrm{z}$ :

$$
v_{x} \equiv U_{k}(x, t)=\int U(x, z, t) e^{+i k z} d z
$$

yields the equation

$$
\frac{\partial}{\partial x}\left[\rho_{0}(x) \frac{\partial^{2}}{\partial t^{2}}-\frac{B_{0}^{2} k^{2}}{\mu_{0}}\right] \frac{\partial U_{k}}{\partial x}-k^{2}\left[\rho_{0}(x) \frac{\partial^{2}}{\partial t^{2}}-\frac{B_{0}^{2} k^{2}}{\mu_{0}}\right] U_{k}=0 .
$$

This equation is now to be solved for $U_{k}(x, t)$, subject to the boundary condition that $U_{k}(x, t)$ vanishes at infinity and to the initial conditions $U_{k}(x, 0)$ and its time derivative $\dot{U}_{k}(x, 0)$. The initial conditions for the velocity themselves must of course satisfy the appropriate boundary conditions and must be physically acceptable, i.e., 
must be sufficiently smooth and square-integrable in the infinite interval.

The Laplace transform of $U_{k}(x, t)$ is defined as usual by the formula

$$
U_{k \omega}(x)=\int_{0}^{\infty} U_{k}(x, t) e^{-i \omega t} d t .
$$

where $\operatorname{Im} \omega>0$. Application of this transformation to Eq. (4.9) gives

$$
\begin{aligned}
& \frac{d}{d x}\left\{\left[\rho_{0}(x) \omega^{2}-\frac{B_{0}^{2} k^{2}}{\mu_{0}}\right] \frac{d U_{k \omega}}{d x}\right\}-k^{2}\left[\rho_{0}(x) \omega^{2}-\frac{B_{0}^{2} k^{2}}{\mu_{0}}\right] U_{k \omega} \\
= & \bar{s}(\omega, x),
\end{aligned}
$$

where $\bar{S}(\omega, x)$ corresponds to the initial conditions. We shall now solve Eq.(4.II) with the aid of Green's functions. As to be noted, the homogeneous Eq. (4.11) is same as Eq. (3.7) discussed in Chapter III. The Green's function $G(x, s ; k, \omega)$ can be expressed in terms of two-linearly independent solutions $U_{k \omega(1)}(x)$ and $U_{k \omega(2)}(x)$ of the homogeneous equation (4.11) satisfies the boundary conditions at $x=-\infty$, and that at $x=-+\infty$. The following formula holds:

$$
\begin{aligned}
G(x, s ; k ; \omega)= & J^{-1}\left[U_{k \omega(1)}(x) U_{k \omega(2)}(x) H(s-x)\right. \\
& \left.+U_{k \omega(2)}(x) U_{k \omega(1)}(s) H(x-s)\right],
\end{aligned}
$$


where $H(x)$ is the unit step function and $J(k, w)$ is the so-called conjunct of the two solutions $U_{k \omega(1)}$ and $U_{k \omega(2)}$ '

$$
J(k, \omega)=\varepsilon(x)\left[\frac{d U_{k \omega(2)}}{d x} U_{k \omega(1)}-\frac{d U_{k \omega(1)}}{d x} U_{k \omega(2)}\right],
$$

with $\varepsilon(x)=\rho_{0}(x) \omega^{2}-\frac{B_{0}^{2} k^{2}}{\mu_{0}}$.

A theorem exists (Theorem (3.2) in Friedman, 1956), which shows that $J(k, \omega)$ is independent of $x$ and $s$.

To make the homogeneous equation (4.11) solvable we choose a monotonic density profile composed of linear sections (Fig. 4.1) such that:

$$
\begin{aligned}
\varepsilon(x) & =\varepsilon_{1}=\omega^{2} \mu_{0} \rho_{1}-k^{2} B_{0}^{2}, x \leq-a(\text { region }(1)) \\
\varepsilon(x) & \left.=\varepsilon_{1}+\Delta\left(\frac{x}{a}+1\right),-a \leq x \leq a \text { (region }(0)\right) \\
\varepsilon(x) & =\varepsilon_{11}=\omega^{2} \mu_{0} \rho_{2}-k^{2} B_{0}^{2}, \quad x \geq a \quad(\text { region }(2)) \\
\text { with } \Delta & =\frac{\omega^{2} \mu_{0}}{2}\left(\rho_{2}-\rho_{1}\right) .
\end{aligned}
$$

With this density profile Eq. (4.11) assumes constant coefficients in the intervals (1) and (2) and becomes a modified Bessel equation in the interval ( 0 ) (the transition region).

The solutions $\mathrm{U}_{\mathrm{k} \omega(1)}$, bounded at $\mathrm{x}=-\infty$, and $\mathrm{U}_{\mathrm{k} \omega(2)}$, bounded at $\mathrm{x}=\infty$, in the three regions can be written as follows: 


$$
U_{k \omega(1)}=\left\{\begin{array}{l}
e^{k(x+a)} \\
A_{1} I_{0}\left(z_{x}\right)+B_{1} K_{0}\left(z_{x}\right) \\
C_{1} e^{-k x}+D_{1} e^{k x}
\end{array}\right.
$$

and

$$
U_{k \omega(2)}=\left\{\begin{array}{l}
A_{2} e^{-k x}+B_{2} e^{k x} \\
C_{2} I_{0}\left(z_{x}\right)+D_{2} K_{0}\left(z_{x}\right) \\
e^{-k(x+a)}
\end{array}\right.
$$

where $z_{x}=-\lambda\left(\omega^{2} \mu_{0} \rho_{x}-k^{2} B_{0}^{2}\right), \lambda=k a / \Delta, I_{0}(z)$ and $k_{0}(z)$ are the modified Bessel functions.

Using the matching conditions, as given in section 2, at the boundaries $x= \pm$ a the constants $A_{1,2}, B_{1,2}, C_{1,2}$ and $\mathrm{D}_{1,2}$ can be decided.

Regardless of whether the solutions in the interval (1), (0) or (2) are used, on substituting the two solutions in (.4.13) we get

$$
\begin{aligned}
J(k, w)= & \frac{a}{\Delta} z_{1} z_{2} D(k, w) \\
D(k, w)= & {\left[I_{0}\left(z_{2}\right)+I_{1}\left(z_{2}\right)\right]\left[K_{0}\left(z_{1}\right)+K_{1}\left(z_{1}\right)\right] } \\
& -\left[I_{0}\left(z_{1}\right)-I_{1}\left(z_{1}\right)\right]\left[k_{0}\left(z_{2}\right)-K_{1}\left(z_{2}\right)\right] .
\end{aligned}
$$


We shall now discuss the properties of $D(k, \omega)$ as a function of $\omega$ in great detail. As it will be evident the study of its analyticity, the structure of its Riemann surface, its zeros and singularities will give the details of the continuum spectrum and the discrete eigenvalues of the differential equation $(4.11)$. Hence, it is appropriate to call the function $D(k, \omega)$ as dispersion function.

The modified Bessel functions $I_{0}(z)$ and $I_{I}(z)$ are entire functions and $\mathrm{K}_{0}(z), \mathrm{K}_{1}(z)$ can be decomposed into a logarithmic term plus entire analytic function $W_{0}(z), W_{I}(z)$ respectively (Erdelyi and Bateman, 1953). Hence, $D(k, \omega)$ may be written as (Sedlacek, 1971)

$$
\begin{aligned}
D(k, w)= & {\left[I_{0}\left(z_{1}\right)-I_{1}\left(z_{1}\right)\right]\left[I_{0}\left(z_{2}\right)+I_{1}\left(z_{2}\right)\right] \ln \frac{z_{2}}{z_{1}} } \\
& -\left[I_{0}\left(z_{1}\right)-I_{1}\left(z_{1}\right)\right]\left[W_{0}\left(z_{2}\right)-W_{1}\left(z_{2}\right)-\frac{1}{z_{2}}\right] \\
& +\left[I_{0}\left(z_{2}\right)+I_{1}\left(z_{2}\right)\right]\left[W_{0}\left(z_{1}\right)+W_{1}\left(z_{1}\right)+\frac{1}{z_{1}}\right] .
\end{aligned}
$$

On writing $\omega_{\mathrm{Al}, 2}=\mathrm{kv}_{\mathrm{Al}, 2}$, the multivaluedness of $D(k, w)$ is determined by the logarithmic term

$$
\ln z_{2} / z_{1}=-\ln \frac{\left(\omega+\omega_{A 1}\right)}{\left(\omega+\omega_{A 2}\right)}+\ln \left(\frac{\omega-\omega_{A 2}}{\omega-\omega_{A 1}}\right)
$$

which shows that $D(k, \omega)$ possesses four logarithmic branch 


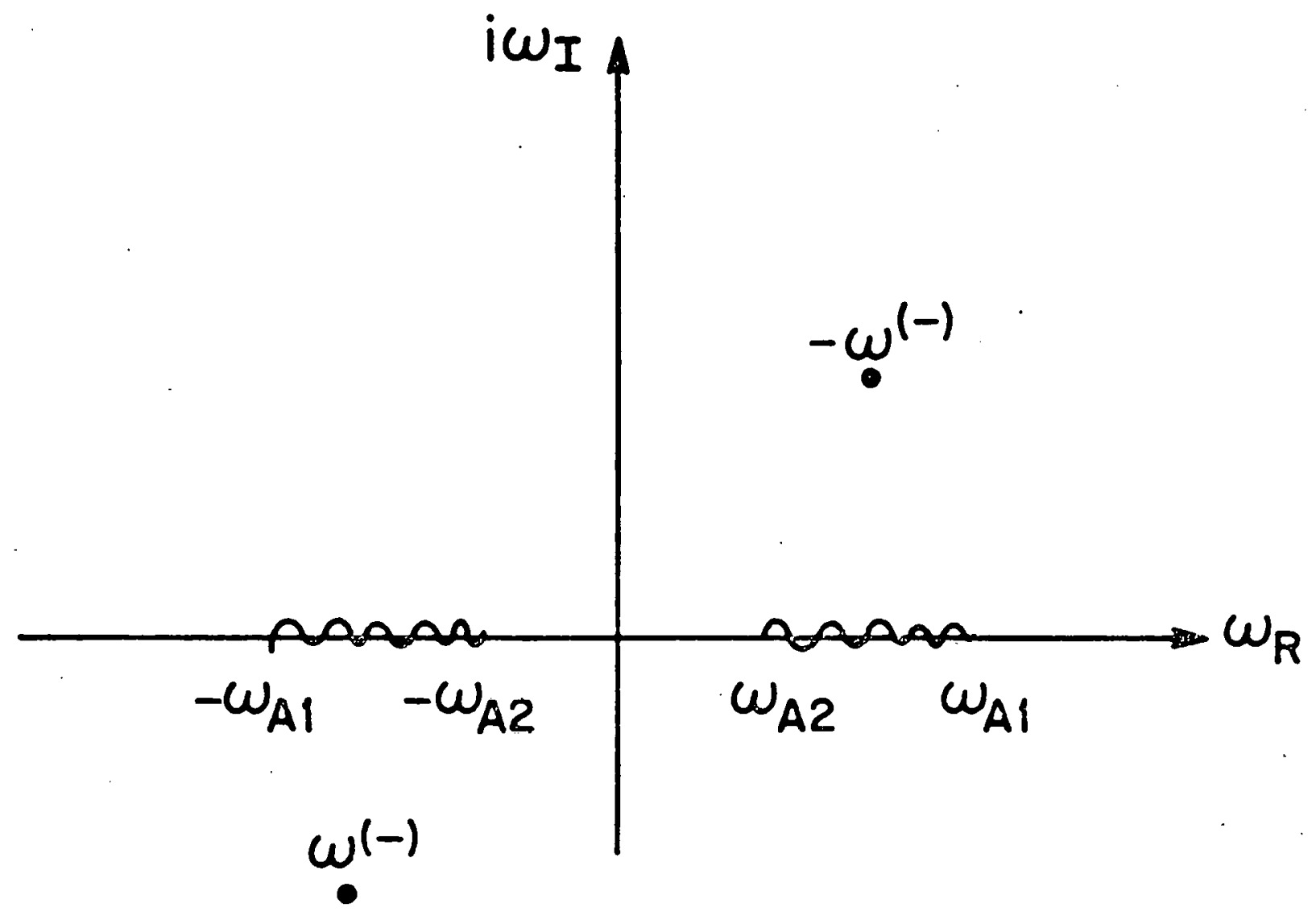

Fig. 4.3 
points on the real axis: $-\omega_{A 2},-\omega_{A 1}, \omega_{A 1}, \omega_{A 2}$ each of which coincides with a simple pole. If the $\omega$-plane is cut along the intervals connecting the first pair and the second pair of these points (Fig.4.3) the function $\ln \left(\mathrm{zn}_{2} / \mathrm{z}_{1}\right)$ becomes a single-valued function of $\omega$. These cuts (or spectral cuts) are identical with the intervals of the continuous spectrum of the differential equation (4.11).

The function $D(k, \omega)$ has the same Riemann surface as the function $\ln \left(z_{2} / z_{1}\right)$. All the branches of $D(k, \omega)$ may then be constructed simply by replacing $\ln \left(z_{2} / z_{1}\right)$ in (4.19) by $\ln \left(z_{2} / z_{1}\right)+2 n \pi i$. The result is

$$
D_{n}(k, \omega)=D_{0}(k, \omega)+2 n \pi i\left[I_{0}\left(z_{1}\right)-I_{1}\left(z_{1}\right)\right]\left[I_{0}\left(z_{2}\right)+I_{1}\left(z_{2}\right)\right] .
$$

We shall now investigate the zeros of $D(k, \omega)$. The zeros lying on the principal sheet of the Riemannian surface are identical with the discrete eigenvalues of the Eq. (4.11). The zeros lying on the d.ther sheets are usually referred to as 'virtual' eigenvalues (because no independently eigenfunctions correspond to them) and are associated with the phenomenon of spectral concentration whose physical interpretation is quite similar to the theory of Landau damping in warm plasma oscillations. 
With the aid of the Nyquist criterion it can be shown that there are no zeros of $D(k, \omega)$ on the principal sheet. There are, however, zeros on the other sheets of the Riemannian surface. We shall calculate those that, at least for small values of the parameter ka (i.e., the leastdamped modes), lie nearest to the real axis which are the ones associated with the phenomenon of spectral concentration. Considering first, the sheet $n=-1$, we expand $D_{-1}(k, \omega)$ in terms of the small parameter ka, retaining only the terms of the zeroth and first order.

$$
\begin{aligned}
D_{-1}(k, \omega)= & -\frac{1}{\lambda}\left\{\left[\frac{1}{\mu_{0} \rho_{1}\left(\omega^{2}-\omega_{A 1}^{2}\right)}+\frac{1}{\mu_{0} \rho_{2}\left(\omega^{2}-\omega_{A 2}^{2}\right)}\right](1+k a)\right. \\
& \left.-\lambda\left(\ln \frac{\rho_{2}\left(\omega^{2}-\omega_{A 2}^{2}\right)}{\rho_{1}\left(\omega^{2}-\omega_{A}^{2}\right)}-2 \pi i\right)\right\} .
\end{aligned}
$$

If $\mathrm{ka}=0$, i.e., if the density profile is discontinuous the dispersion function $D(k, \omega)$ gives the dispersion relation representing surface modes, as derived in section 2,

$$
\rho_{2}\left(\omega^{2}-\omega_{A l}^{2}\right)+\rho_{1}\left(\omega^{2}-\omega_{A l}^{2}\right)=0 \text {. }
$$

The roots of this equation are purely real, therefore we can assume that the imaginary part of the solution of Eq. (4.22) will be of order ka. We take $\omega=\omega_{R}+i \omega_{I}$, and then retaining 
only the terms of zeroth and first order in ka, we split the Eq. (4.22) into real and imaginary parts, which gives the following two equations to determine $\omega_{R}$ and $\omega_{I}$ :

$$
\begin{aligned}
& {\left[\frac{1}{\left(\omega_{R}^{2}-\omega_{A 1}^{2}\right) \rho_{1}}+\frac{1}{\left(\omega_{R}^{2}-\omega_{A 2}^{2}\right) \rho_{2}}\right]+k a\left[\frac{1}{\left(\omega_{R}^{2}-\omega_{A 1}^{2}\right) \rho_{1}}\right.} \\
& \left.+\frac{1}{\left(\omega_{R}^{2}-\omega_{A 2}^{2}\right) \rho_{2}}\right]+\frac{2 k a}{\left(\rho_{2}-\rho_{1}\right) \omega_{R}^{2}}\left[\ln \frac{\rho_{2}}{\rho_{1}}+\ln \frac{\omega_{R}^{2}-\omega_{A}^{2}}{\omega_{R}^{2}-\omega_{A}^{2}}\right]=0,
\end{aligned}
$$

and

$$
-2 \omega_{I} \omega_{R}\left[\frac{1}{\left(\omega_{R}^{2}-\omega_{A 1}^{2}\right)^{2} \rho_{1}}+\frac{1}{\left(\omega_{R}^{2}-\omega_{A 2}^{2}\right)^{2} \rho_{2}}\right]+\frac{4 \mathrm{ka}}{\left(\rho_{2}-\rho_{1}\right) \omega_{R}^{2}}=0 \text {. }
$$

We see from (4.25) that the effect of a continuous density profile $(k a \neq 0)$ is to introduce damping to the originally undamped surface wave modes of a discontinuous $(k a=0)$ MHD plasma.

We denote the roots on $\mathrm{n}=-1$ sheet as $\omega^{(-)}$. Since the dispersion relation is a function of only $\omega^{2}$, the point $-\omega^{(-)}$is also a root. The roots on $n=1$ sheet, $\omega^{(+)}$ and $-\omega^{(+)}$can be found in the same way. 
Considering the oscillation frequency $\omega_{R}^{(-)}$to be given approximately by the Eq. (4.23) we have

$$
\omega_{R}^{(-)} \simeq \sqrt{2} k \frac{B_{0}}{\mu_{0}\left(\rho_{1}+\rho_{2}\right)} .
$$

Substituting in (4.26) this value of $\omega_{R^{\prime}}$ we get the damping rate $\omega_{I}$ as :

$$
\frac{\omega^{(-)}}{\omega_{R}^{(-)}} \simeq-\left(\frac{\pi}{4}\right) \quad(2 \mathrm{ka}) \cdot \frac{\left(\rho_{1}-\rho_{2}\right)}{\left(\rho_{1}+\rho_{2}\right)} \text {. }
$$

If $\rho_{2} \ll \rho_{1}$, we have

$$
\omega_{\mathrm{R}}^{(-)} \simeq \sqrt{2} k \mathrm{v}_{\mathrm{Al}} \text { and } \frac{\omega \frac{(-)}{I}}{\omega_{\mathrm{R}}^{(-)}} \simeq-\frac{\pi}{2}(\mathrm{ka}) .
$$

Here $a \simeq[d \ln \rho / d x]_{x=0}^{-1}$ is the scale length of the density variation. We note, therefore, that sharper the density discontinuity is, i.e., the smaller $\mathrm{ka}$ is, weaker the damping is.

'the complete solution of (4.9) will be given by performing inverse Laplace transform which then gives $U(x, t)$ in terms of the Bromwich integral

$$
U_{k}(x, t)=\frac{1}{2 \pi} \int_{C} u_{k \omega}(x) e^{-i \omega t} d \omega
$$

where the integration path $C$ runs parallel to the real axis of the plane above all singularities of $\mathrm{U}_{\mathrm{k} \omega}(\mathrm{x})$. 
Without going into complete details of finding the asymptotic behavior, we give the results as obtained by Sedlaček (1971)

$$
\begin{aligned}
& U_{k}(x, t)=A_{1}(x ; k) t^{-1} e^{-i \omega_{A}(x) t}+A_{2}(x ; k) t^{-1} e^{i \omega_{A}(x) t} \\
& +B_{1}(x ; k) e^{-i \omega(-)} t+B_{2}(x ; k) e^{-i \omega(t)} t \text {. }
\end{aligned}
$$

The first two terms in (4.25) correspond to the noncollective modes [the continuous spectrum $S=\left\{\omega\left|\min \omega_{A}^{2} \leq \omega^{2} \leq \operatorname{man} \omega_{A}^{2}\right|\right\}$ ] with asymptotically, position dependent frequency $\omega_{A}(x)$ and damping proportional to inverse power of time. The second two terms, though negligible in the asymptotic expansion (4.30), represent the discrete spectrum, corresponding to the only collective modes of oscillations (the surface eigenmodes) with position independent frequency and exponential damping (resonant absorption). The damping can be rather weak if the plasma parameters have sharp variations. The damped surface eigenmode is used by Chen and Hasegawa (1974) to explain the impulse excited damped magnetic pulsations.

In the next section we elucidate the physical mechanism of the resonant absorption using kinetic theory. This absorption is a manifestation of the resonant mode conversion of the surface wave to the kinetic Alfvên wave introduced in Section 2.6 . 


\subsection{Resonant Mode Conversion}

In order to understand the energy absorption

mechanism we shall study the linear transformation of the Alfvén surface wave to the kinetic Alfvén wave in the neighborhood of the resonance region (Hasegawa and Chen, 1976).

The logarithmic singularity in the solution of Alfvén wave equation arises because the ideal MHD equations do not have any dissipative mechanism and/or assume zero Larmour radius. This singularity is seen to be removed when finite resistivity effect or finite Larmour radius correction is introduced in the ideal MHD equations. The effect of resistivity on the Alfvén surface wave has been considered by Kappraff and Tataronis (1977) and Uberoi (1978). This treatment, however, is valid only when the plasma skin depth is shorter than the ion gyroradius. We note that finite resistivity effects are smaller than kinetic effects by $0\left(\nu_{e i} v_{A}^{2} / \omega_{0} v_{T e}^{2}\right)$, where $\nu_{e i}$ is the electron-ion collision frequency, and $\omega_{0}$ is either the applied frequency or the eigenfrequency of the surface wave. For a typical hot plasma, $v_{\mathrm{Te}}^{2} / \mathrm{v}_{\mathrm{A}}^{2}=\left(\beta \mathrm{m}_{\mathrm{i}} / \mathrm{m}_{\mathrm{e}}\right) \gg 1$, and $v_{\mathrm{e} i} \leqslant 10^{5} / \mathrm{sec}$ and $\omega_{0} \sim 10^{6} \mathrm{rad} / \mathrm{sec}$. Therefore, the skin depth is generally much larger than the ion gyroradius. Then the kinetic Alfvén wave excited at the singular surface can propagate for considerable distance measured by the size of ion gyroradius and the energy is dissipated more uniformly in the plasma. 
This phenomenon cannot be seen by the one fluid MHD theory, but can be studied only by the kinetic theory. We consider the unperturbed distribution function for the ions to be given by

$$
f_{i}^{(0)}=g\left(x+\frac{v_{y}}{\omega_{c i}}\right) f(0){\underset{\sim}{v})}_{(v)}
$$

with $f^{0}(v)$ being the Maxwell distribution with the ion temperature $T_{i}$, and $g$ being a function representing the plasma nonuniformity in the $\mathrm{x}$ direction.

The linearized vlasov equation for the perturbed distribution function of ions is

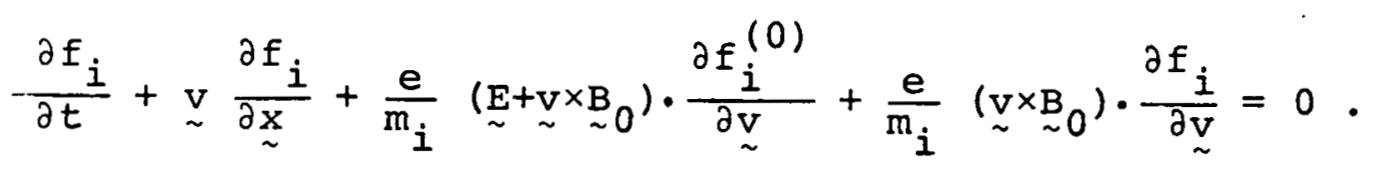

The perturbed distribution function of electrons obey the drift kinetic equation

$$
\frac{\partial f}{\partial t}+v_{z} \frac{\partial f_{e}}{\partial z}+\left(\frac{E_{y}}{B_{0}}+v_{z} \frac{B_{x}}{B_{0}}\right) \frac{\partial f_{e}^{(0)}}{\partial x}-\frac{e}{m_{e}} E_{z} \frac{\partial f_{e}^{(0)}}{\partial v_{z}}=0 .
$$

The field equations are same as derived in Chapter II (Eqs. (2.33) and (2.34)); the quasi-neutrality condition, 


$$
\mathrm{n}_{i}=\mathrm{n}_{\mathrm{e}}
$$

and Ampére's law in the $z$ direction

$$
\frac{\partial}{\partial z} \nabla_{\perp}^{2}(\phi-\psi)=\mu_{0} \frac{\partial}{\partial t}\left(J_{i z}+J_{e z}\right)
$$

In these expressions, the number density $\mathrm{n}$ and the current density $J$ are given by the distribution function $f$

$$
n_{j}=n_{0} \int f_{j} d v, j=i, e,
$$

and

$$
J_{j}=q_{j} n_{0} \int v f_{j} d v, j=i, e .
$$

The set of equations from $(4.31)$ to $(4.37)$ describes the electromagnetic waves in a magnetohydrodynamic frequency range without restrictions on the size of the perpendicular wavelength (but with a restriction of no compressional magnetic field perturbation).

To study the resonant mode conversion of the Alfvén wave, it is convenient to assume that $\mathrm{k}_{y^{\prime}}$, the wavenumber perpendicular to the magnetic field as well as to the density gradient, is much smaller than $\rho_{i}^{-1}$ and that the scale size of the density gradient is much larger than the ion gyroradius so that

$$
\omega / \omega_{c i} \gg k_{y} k \rho_{i}^{2},
$$

where $\kappa^{-1}$ is the inhomogeneity scale. 
This assumption allows us to ignore the term $v_{y} / \omega_{c i}$ in $g$ in Eq. (4.31) which is equivalent to the neglection of a drift wave.

Finally, we assume that the wavelength in the $x$ direction near the mode conversion point is small but larger than the ion gyroradius so that we can expand the wave equation in the power of $\rho_{i} d / d x$.

With these assumptions the Fourier amplitude of the number density and the current density perturbations are given by

$$
\begin{aligned}
& \frac{e n_{i}}{\varepsilon_{0}}=\frac{\omega_{p i}^{2}}{\omega_{c i}^{2}}\left[\frac{d}{d x}\left(1+\frac{3}{4} \rho_{i}^{2} \frac{d^{2}}{d x^{2}}\right)\left(g \frac{d \phi}{d x}\right)-g k_{y}^{2} \phi\right]+\frac{\omega_{p i}^{2}}{\omega^{2}} k_{z}^{2} g \psi, \\
& \frac{e n_{e}}{\varepsilon_{0}}=\frac{\omega_{p e}^{2}}{v_{T e}^{2}} g \psi, \\
& \mu_{0} J_{i z}=\frac{\omega_{p i}^{2}}{c^{2}} \frac{k_{z}}{\omega} g \psi
\end{aligned}
$$

and

$$
\mu_{0} J_{e z}=-\frac{\omega_{\mathrm{pe}}^{2}}{c^{2} v_{\mathrm{Te}}^{2}} \frac{\omega}{k_{\mathrm{z}}} \mathrm{g} \psi .
$$


In these expressions, the ion and the electron plasma frequencies, $\omega_{\mathrm{pi}}$ and $\omega_{\mathrm{pe}}$ are constants and refer to the values at the maximum plasma density where $g(x)$ is normalized to unity.

To study the resonant mode conversion to the kinetic Alfvén wave, we only consider the region near the resonant point $x=x_{0}$ where $\omega^{2}=k_{z}^{2} v_{A}^{2} / g\left(x=x_{0}\right)$. We can then further simplify the expressions using the assumption of a low $B$ plasma so that $v_{A}^{2}>c_{S}^{2}=T_{e} / m_{i}$. This assumption eliminates the possibility of a simultaneous coupling to the ion acoustic wave. The wave equation can then be derived by eliminating $\psi$ from Eqs. $(4.34),(4.35)$ and $(4.38)$ to $(4.41)$,

$$
\begin{aligned}
& \left(\frac{\omega^{2}}{k_{z}^{2} v_{A}^{2}} \frac{3}{4} \rho_{i}^{2} \frac{d^{3}}{d x^{3}}+\frac{d^{2}}{d x^{2}} \frac{1}{g} \frac{T_{e}}{T_{i}} \rho_{i}^{2} \frac{d}{d x}\right)\left(g \frac{d \phi}{d x}\right) \\
& +\left[\frac{d}{d x}\left(\frac{\omega^{2}}{k_{z}^{2} v_{A}^{2}} g-1\right) \frac{d}{d x}-k_{y}^{2}\left(\frac{\omega^{2}}{k_{z}^{2} v_{A}^{2}}-1\right)\right] \phi=0
\end{aligned}
$$

where the Alfvén speed $v_{A}^{2}$ is that of the maximum density and $g$ is normalized to unity.

We can immediately notice that if we put $\rho_{i}+0$ in this expression, the wave equation reduces to

$$
\frac{d}{d x}\left[\varepsilon(x) \frac{d \phi}{d x}\right]-k_{y}^{2} \varepsilon(x) \phi=0,
$$

here $\varepsilon(x)=\frac{\omega^{2}}{k_{z}^{2} v_{A}^{2}} g-1$. 
Equation (4.43) has $w$ structure identical to the magnetohydrodynamic wave equation (4.11). It is also noted that in a uniform plasma, $\mathrm{g}=1$, Eq. (4.42) gives two decoupled wave equations

$$
\nabla_{1}^{2} \phi=0
$$

and

$$
\left[\rho^{-2} \frac{\mathrm{d}^{2}}{\mathrm{dx}^{2}}+\left(\frac{\omega^{2}}{\mathrm{k}_{\mathrm{z}}^{2} \mathrm{v}_{\mathrm{A}}^{2}}-1\right)\right] \phi=0,
$$

where $\rho^{-2}=\left(\frac{3}{4}+\mathrm{T}_{\mathrm{e}} / \mathrm{T}_{\mathrm{i}}\right) \rho_{\mathrm{i}}^{2} \cdot \mathrm{k}_{\mathrm{y}} \ll \mathrm{d} / \mathrm{dx}$ is used in Eq. (4.45). Equation (4.44) represents a quasi-static electromagnetic perturbation (cut-off mode) associated with an external source. In the absence of a source, this equation represents a surface wave. Equation (4.45) is the wave equation for the bulk kinetic Alfvén wave, Eq. (2.44).

We can thus identify that Eq. (4.42) represents a coupling between a surface magnetohydrodynamic mode or an externally applied electromagnetic perturbation and the kinetic Alfvén wave. From Eq. ( 4.45$)$, we can see that the kinetic Alfvén wave propagates, after the mode conversion, to the higher density side where $\mathrm{k}_{\mathrm{z}}^{2} \mathrm{v}_{\mathrm{A}}^{2}(\mathrm{x})<\mathrm{k}_{\mathrm{z}}^{2} \mathrm{v}_{\mathrm{A}}^{2}\left(\mathrm{x}=\mathrm{x}_{0}\right)$. 
To study the mode conversion, we must specify the actual density profile. Because the wave after the mode conversion is expected to propagate on the higher density side, the solution depends on whether the converted wave can propagate all the way across the plasma column or dissipates significantly before it reaches the other side. We assume here that the plasma to be semi-infinite in the $\mathrm{x}$ direction.

As a simple example we take a linear profile for the plasma density such that

$$
g(x)=k x+a,
$$

where $\mathrm{x}$ is a normalized distance whose origin is located at the resonant point where $g(x=0) \omega^{2} / k_{z}^{2} v_{A}^{2}=1$, or $a \omega^{2} / k_{z}^{2} v_{A}^{2}=1$, with $0<\mathrm{a}<1$. Equation $(4.42)$ is then reduced near $\mathrm{x} \sim 0$ to a simple form

$$
\rho^{2}\left(d^{2} E_{x} / d x^{2}\right)+K x E_{x}=E_{0}
$$

where

$$
\rho^{2}=\left(\frac{3}{4}+\frac{k_{z}^{2} v_{A}^{2}}{\omega^{2}} \frac{T_{e}}{T_{i}}\right) \rho_{i}^{2}
$$

and

$$
E_{\mathbf{x}}=-\partial \phi / \partial \mathbf{x}
$$

$E_{0}$ is an integration constant representing a nominal. value of $E_{x}$ at a large negative $x$ ( $E_{x}$ associated with the external source field or surface wave). 
In the derivation we also assumed that $|\mathrm{dg} / \mathrm{dx} / \mathrm{g}| \ll|\mathrm{d} \phi / \mathrm{dx} / \phi|$; that is, the variation of the wave amplitude is much faster than the variation of the density (WKB approximation). The wave equation of the type of Eq. ( 4.46 ) has been studied extensively in relation to the mode conversion of the electromagnetic wave to the Langmuir wave. It is well.known that the solution can be expressed in terms of the Airy functions.

If we introduce a scale length

$$
\Delta=\left(\rho^{2} / \kappa\right)^{1 / 3}
$$

and use a normalized electric field intensity

$$
E_{x}=-\frac{E_{x}}{E_{0}} \frac{(K \rho)^{2 / 3}}{\pi},
$$

the general solution is given by

$$
\bar{E}_{x}=c_{1} A_{i}(-x / \Delta)+c_{2} B_{i}(-x / \Delta)+G_{i}(-x / \Delta)
$$

where $A_{i}, B_{i}$ are $A i r y$ functions and $G_{i}$ is a function involving integrals of $A_{i}$ and $B_{i}$.

The integration constants $c_{1}$ and $c_{2}$ can be decided by applying appropriate boundary conditions. For a semiinfinite plasma as considered here, they are decided whether or not the energy source exist internal or external to the plasma. 
For the case of the resonant absorption or the surface wave damping as discussed in section 3.3 and 4.3, the suitable boundary condition for the kinetic Alfven wave is to accept only the right-going wave (no reflection at $x \rightarrow \infty$ ) and that which has no divergence at $x \rightarrow-\infty$. We can then 


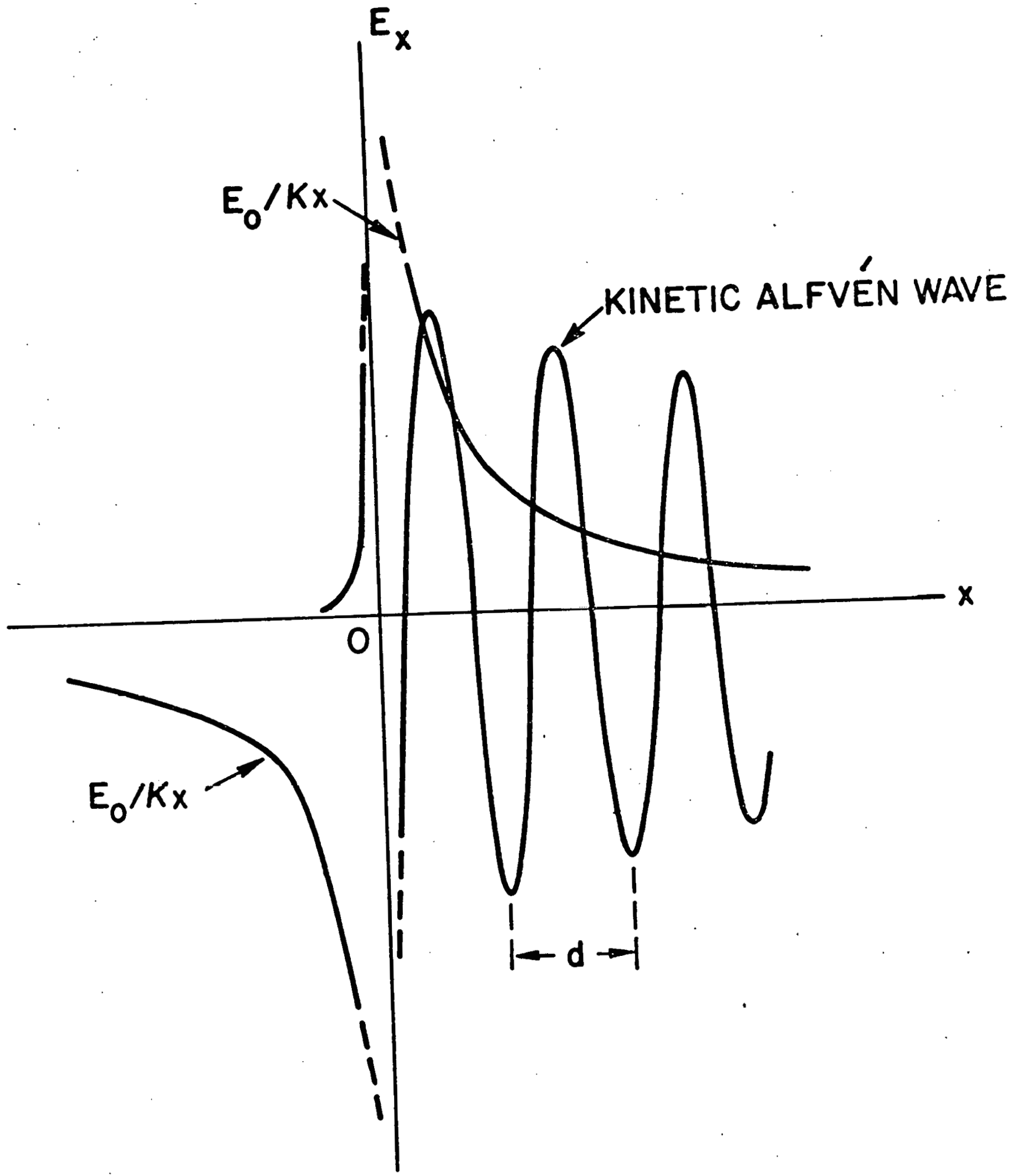

Fis. 4.4 
find $c_{2}=0$ and $c_{1}=i$. The asymptotic solution for $|x / \Delta| \gg>1$ can then be written as

$$
E_{x}=-\frac{\pi^{1 / 2} E_{0}}{(\kappa \rho)^{2 / 3}}\left(\frac{\Delta}{x}\right)^{1 / 4} \exp \left\{i\left[\frac{2}{3}\left(\frac{x}{\Delta}\right)^{3 / 2}+\frac{\pi}{4}\right]\right\}+\frac{E_{0}}{\kappa x}
$$

for $x>0$,

$$
E_{x}=E_{0} / K x
$$

for $x<0$. The first term in Eq. (4.52) represents the kinetic Alfvén wave and the second term as well as the expression in Eq. (4.53) the source or surface wave field.

As is expected, the kinetic Alfvén wave propagates on the higher density side $(x \geq 0)$ of the resonant point as can be seen in Eq. $(4.52)$. In Eq. (4.52) it is worth noting that the first few peak amplitudes of the kinetic Alfvén wave after the mode conversion are given by $E_{0}(K \rho)^{-2 / 3}$ with an effective wavenumber of $\left(\kappa / \rho^{2}\right)^{1 / 3}$, while away from the resonant point, say at $x \sim \kappa^{-1}$, the amplitude and the wavenumber of the kinetic Alfvén wave become $E_{0}(\kappa \rho)^{-1 / 2}$ and $\rho^{-1}$, respectively. The qualitative feature of the mode converted kinetic Alfvén wave is shown in Fig. 4.4. We can see that the solution for $x<\theta$ is identical to the one obtained under the ideal magnetohydrodynamic 
approximation. Hence, the plasma impedance, and consequently the absorption rate, seen from the external circuit remains unchanged from the previous magnetohydrodynamic calculation.

If the energy source exist $\$$ inside the plasma, we must $V$ take the left going wave also. Such a problem is important in the analysis of the drift Alfvén instability, section 5.5 . 


\section{REFERENCES FOR CHAPTER IV}

Baldwin, D.E. and Ignat, D.W., Resonant absorption in zerotemperature nonuniform plasma, Phys. Fluids, 12, 697 (1969).

Chen, L. and Hasegawa, A., A theory of long period magnetic pulsations, 2. Impulse excitation of surface eigenmode, J. Geophys. Res. 79, 1033 (1974).

Erdelyi, A. and Bateman, H., Higher Transcendental Functions, Vol. II, New York, McGraw-Hill Book Co, 1953.

Friedman, B., Principles of Techniques of Applied Mathematics, New York, John Wiley and Sons, 1956. Golant, V.E. and Piliya, A.D., Linear transformation and absorption of waves in a plasma, Sov. Phys. Uspekhi 14 , $413(1972)$.

Grossmann, W., Kaufmann, M. and Neuhauser, J., Damping of.

Alfvên and magneto-acoustic waves at high beta, Nuclear Fusion 13, 462 (1973).

Hasegawa, A., Plasma Instability and Nonlinear Effects, Springer, New York, 1975.

Hasegawa, A. and Chen, L., Kinetic processes in plasma heating by resonant mode conversion of Alfvén wave, Phys. Fluids 19, 1924 (1976).

Kappraff, J.M. and Tataronis, J.A., Resistive effects on Alfvén wave heating, J. Plasma Physics 18, 209 (1977). Kruskal, M. and Schwarzschild, M., Some instabilities of a completely ionized plasma, Proc. Roy. Soc. (London) A223, 348 (1954). 


$$
\begin{aligned}
& -119- \\
& I V-2-
\end{aligned}
$$

Pritchett, P.L. and Dawson, J.M., Phase mixing in the continuous spectrum of Alfvén waves, Phys. Fluids 21 , 516 (1978).

Sedlácek, Z., Electrostatic oscillations in cold inhomogeneous plasma, 1. Differential equation approach, J. Plasma Physics $\underline{5}, 239$ (1971).

Uberoi, C., A note on resonant absorption of Alfvén waves in nonuniform plasma, Proc. Kiev Conference on Plasma Physics, Trieste, April (1977). 


\section{OTHER REFERENCES OF CHAPTER IV}

Chen, Liu and Hasegawa, A., A theory of long-period magnetic pulsations. I. Steady state excitation of field line resonance. J. Geophys. Res. 79, 1024-32 (1974).

Cummings, W.D., O'sullivan, R.J. and Coleman, P.J. Jr., Standing Alfvén waves in the magnetosphere. J. Geophys. Res. $74,778-93$ (1969).

Davies, B., Alfvén waves in a non uniform resistive plasma. Phys. Rev. Lett. 22, $1246-7$ (1969).

Deutsh, R.V., Propagation of Alfvén waves in nonhomogeneous plasma. Rev. Roumaine Phys. 13, 673-81 (1968).

Gerwin, R., Hydromagnetic surface waves in a conducting liquid surrounded by a compressible gas, Phys. Fluids 10, 2164 (1967).

Grossmann,, . and Tataronis, J., Decay of MHD waves by phase mixing. II. The theta-pinch in cylindrical geometry. Z. Phys. 261, 217-36 (1973).

Grossman, W., Kaufmann, M. and Neuhauser, J., Damping of Alfvén and magneto-acoustic waves at high beta. Nucl. Fusion 13, 462-4 (1973).

Morrow R. and Brennan, M.H., Compressional Alfvén waves in

a non-unitorm plasma. Plasma Phys. (GB) 13, 75-9 (1971). Swanson, D.G., Mode conversion of toroidal Ifvèn waves. Phys. F1 uids 18, 1269-76 (1975).

Swanson, D.G., Mode conversion and tunneling at the twoion hybrid resonance. Phys. Rev. Lett. 36, 316-19 (1976). 


\section{PLASMA INSTABILITIES \\ ASSOCIATED WITH THE ALFVÉN \\ WAVE}

\subsection{Introduction}

In this chapter, we discuss plasma instabilities associated with the excitation of Alfvén waves. As many other cases of plasma instabilities, the Alfvén wave becomes unstable both due to the velocity space nonequilibrium and coordinate space nonequilibrium. These instabilities appear by a conversion of free energy stored in the plasma to the Alfvén perturbation. The free energy exists when the velocity distribution of the plasma particles is not an isotropic Maxwellian or when the plasma is not uniform in space. The former case, a case of velocity space nonequilibrium produces an instability called velocity space instability, while the other case, the case of coordinate space nonequilibrium produces an instability called coordinate space instability. The instabilities work to equilibrate the distribution. The instability is found by solving the dispersion relation for the eigenfrequency $\omega$ for a given value of the wave number $k$. For an assumed Fourier amplitude expression of exp $i(\underset{\sim}{k} \cdot \underset{\sim}{x}-\omega)$, the instability exist when $\omega$ has a positive imaginary part. We shall present some of the typical Alfvén wave instabilities. 


\subsection{Fire Hose Instability}

Here, we present an instability when the plasma has an anisotropic pressure. If we take the direction of $\mathrm{k}$ vector in $\mathrm{x}-\mathrm{z}$ plane, where $\mathrm{z}$ is the direction of the ambient magnetic field, the Alfvén wave dispersion relation is written by

$$
\frac{c^{2} k_{z}^{2}}{\omega^{2}}-\varepsilon_{x x}=0
$$

here $\varepsilon_{x x}$ is the $x x$ component of plasma dielectric constant, and is listed in the Appendix. In the MHD frequency $\left(\omega / \omega_{\mathrm{Ci}} \ll 1\right)$ and the wave number $\left(k v_{\mathrm{Ti}} / \omega_{\mathrm{Ci}} \ll 1\right)$ range $\varepsilon_{\mathrm{Xx}}$ is given by

$$
\begin{aligned}
\varepsilon_{x x} & =\sum_{j} \frac{\omega_{p j}^{2}}{\omega_{c j}^{2}}\left[\left\langle\left(1-\frac{k_{z} v_{z}}{\omega}\right)^{2}\right\rangle_{j}\right. \\
& \left.-\frac{k_{z}^{2}\left\langle v_{1}^{2}\right\rangle}{2 \omega^{2}}\right]
\end{aligned}
$$

where the summation $j$ is over different species (electrons and ions), $v_{1}$ is the velocity in the direction perpendicular to the amblent magnetic field and < > indicates average. Hence, the dispersion relation of the Alfvén wave becomes 


$$
\frac{\omega^{2}}{\mathrm{k}_{\mathrm{z}}^{2} \mathrm{v}_{\mathrm{A}}^{2}}=1-\frac{1}{2}\left(\beta_{\|}-\beta_{1}\right)
$$

where, $\beta_{\|}$and $\beta_{1}$ are the parallel and perpendicular component of the ratio of the plasma pressure to the magnetic field pressure,

$$
\beta_{\perp}=\sum_{j} \frac{1}{2} \frac{m_{j}^{n<v_{1}^{2}>} j}{B_{0}^{2} / 2 \mu_{0}}, \quad B_{\|}=\sum_{j} \frac{m_{j}^{\left.n<v_{z}^{2}\right\rangle}}{B_{0}^{2} / 2 \mu_{0}} .
$$

From Eq. (5.3), it can be easily seen that when $\beta_{\|}-\beta_{\perp}>2$, $\omega$ becomes pure imaginary, hence the instability appears. Because the physical mechanism of this instability is similar to that which generates oscillations in the water hose when the water pressure exceeds a critical value, this is called fire hose instability. (Rosenbluth, 1956; Parker, 1958)

\subsection{Two Stream Instability}

When there exists a stream of charged particles in the plasma, the Alfvén wave becomes unstable. To analyze this instability, we cannot use the dispersion relation of the Alfvén wave, (5.1), because the existence of streaming particle produces a nonvanishing $\varepsilon_{x y}$ component. For simplicity we consider the wave propagating in the direction of the ambient magnetic field. Then the wave equation can be written, 


$$
\left[-k^{2}+\frac{\omega^{2}}{c^{2}}\left(1+\varepsilon_{x x}\right)-i \frac{\omega^{2}}{c^{2}} \varepsilon_{x y}\right] E_{L}=0
$$

Since the Alfven wave is the lower frequency.limit of the ion cyclotron wave which is left-hand polarized, we have introduced a left-hand polarized electric field vector defined by $E_{L}=E_{x}+i E_{y}$.

For a low $\beta$ plasma the dispersion relation can be written by using $\varepsilon_{x y}$ in the Appendix as

$$
\mathrm{k}^{2}-\frac{\omega^{2}}{v_{\mathrm{A}}^{2}}+\frac{\omega_{\mathrm{ps}}^{2}}{\mathrm{c}^{2} \omega_{\mathrm{ce}}}\left(\omega-\mathrm{kv}_{0}\right)=0
$$

or alternatively,

$$
\left(\frac{\omega}{\omega_{C i}}-\frac{\alpha}{2}\right)^{2}-\left(\frac{k v_{A}}{\omega_{C i}}-\frac{\alpha v_{0}}{2 v_{A}}\right)^{2}=\frac{\alpha^{2}}{4}\left(1-\frac{v_{0}^{2}}{v_{A}^{2}}\right),
$$

where $\alpha$ is a ratio of streaming electron density to the plasma density, $v_{0}$ is the velocity of the electron stream. It is easily seen that the instability occurs for $k=\alpha v_{0} \omega_{C i} / 2 v_{A}^{2}$ and the threshold condition is $\mathrm{v}_{0}>\mathrm{v}_{A}$. The interesting point of this instability is that the frequency at which the instability occurs is given by $\alpha \omega_{c i} / 2$, that is, it is proportional to the density ratio of the stream to the plasma. There 
exists another type of two-stream instability in the Alfvén wave, when the finite Larmour radius correction is included. In this case, the kinetic Alfvén wave is excited by electrostatic coupling which requires a density perturbation. The instability can be obtained by using the dispersion of the kinetic Alfvên wave and by including an additional term which originates from the streaming particles. The instability can be created by streaming ions also in Tokamak (Rosenbluth and Ruther ford 1975). 5.4 Kelvin-Helmholtz Instability

When there exists a velocity shear, the KelvinHelmholtz instability appears also in a plasma. In the MHD frequency range the instability excites the Alfven wave.

For simplicity we assume two types of plasma, one is stationary and exists at $x<0$ (medium 1 ) and the other which exists at $x>0$ (medium 2) and moves at the velocity $v_{0}$ with respect to the former. $x=0$ is the boundary surface between these two plasmas. We consider a wave which propagates along this surface as $\exp i\left(k_{y} y+k_{z} z-\omega t\right)$, where $z$ is the direction of the ambient field. The analysis is similar to that in section 4.2 of Chapter IV, except that one $\omega$ is Doppler shifted with repsect to another by $\underset{\sim}{\mathrm{k}} \underset{\sim}{V_{0}} \cdot$ The dispersion relation is then modified from Eq. (4.6),

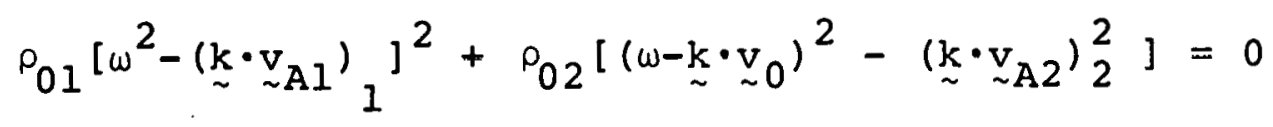


where the vector Alfvén velocity ${\underset{\sim A}{A}}_{A}$ is taken in the direction of the magnetic field. The condition of instability can then be obtained from the condition of a complex root of $w$ as (Chandrasekhar, 1961)

$$
\left(\underset{\sim}{k} \cdot v_{0}\right)^{2}>\left(\frac{1}{\rho_{0 I}}+\frac{1}{\rho_{02}}\right)\left[\rho_{0 I}\left(\underset{\sim}{\left(k \cdot v_{\sim}\right.}\right)_{I}^{2}+\rho_{02}\left(\underset{\sim}{\left(k \cdot v_{A}\right)}\right)_{2}^{2}\right] .
$$

We see that the instability is most easily excited when the plasma flow $\underset{\sim}{\mathrm{v}_{0}}$ is perpendicular to the ambient magnetic field and for the wave which propagates in the direction of the flow. The excited wave is eliptically polarized.

\subsection{Drift Alfvén Wave Instability}

In an inhomogeneous plasma the dispersion relation of the Alfven wave is modified by the diamagnetic drift of 
ions. If we ignore the finite Larmour radius effect, the dispersion relation becomes,

$$
\omega^{2}+\omega \omega_{i}^{*}-k_{z}^{2} v_{A}^{2}=0
$$

where $\omega_{i}^{*}$ is the ion diamagnetic drift frequency

$$
\omega_{i}^{*}=\frac{\left(\underset{\sim}{k} \times()_{\sim}\right) \cdot \hat{z} v_{\mathrm{Ti}}^{2}}{\omega_{c i}},
$$

where $k$ is the measure of the density gradience

$$
\underset{\sim}{\kappa}=\nabla \ell n \mathrm{n}_{0}
$$

The finite Larmour radius correction produces the coupling to the electrostatic electron drift wave. The local dispersion relation for $\omega>\mathrm{k}_{\mathrm{z}} \mathrm{v}_{\mathrm{Ti}}$ then becomes, (Mikhailovskii, 1967)

$$
\begin{aligned}
\left(\omega^{2}+\omega \omega_{i}^{*}-k_{z}^{2} v_{A}^{2}\right) & \left(1-\frac{\omega_{e}^{*}}{\omega}\right)\left(1+i \sqrt{\frac{\pi}{2}} \frac{\omega}{k_{z} v_{T e}}\right) \\
& =\frac{T_{e}}{T_{i}} \lambda_{i} k_{z}^{2} v_{A}^{2}\left(1+\frac{\omega_{i}^{*}}{\omega}\right),
\end{aligned}
$$

where $\lambda_{i}=\left(k_{\perp} v_{T i} / \omega_{C i}\right)^{2}$.

The Alfvén wave becomes unstable if $2 \mathrm{k}_{z} \mathrm{v}_{A}<\omega_{i}^{*}$, with the frequency $\omega$ and the growth rate $\gamma$ given respectively by

$$
\omega \simeq \frac{\mathrm{k}_{\mathrm{z}}^{2} \mathrm{v}_{\mathrm{A}}^{2}}{\omega_{i}^{*}},
$$


and

$$
\gamma=\lambda_{i} \sqrt{\frac{\pi}{2}} \frac{k_{z}^{4} v_{A}^{4}}{\omega_{i}^{\star} 2} \cdot \frac{1}{k_{z} v_{\mathrm{Te}}}
$$

Note that the Alfvén frequency is reduced in this case. The dispersion relation is obtained by assuming $\mathrm{k}_{\mathrm{x}}=0$, where $\mathrm{x}$ is the direction of the density gradient. To include the local effect, one must solve the eigenvalue problem for a wave equation in the $\mathrm{x}$ direction. Such an analysis gives results which distinguish whether the instability is convective (spatial growth in $x$ ) or absolute (temporal growth). This is a problem of recent controversy. Tsang et al.'s (1978) analysis shows that the instability is convective.

If it is convective, the wave energy may escape from the plasma in a manner exactly opposite from the resonant mode conversion as discussed in section 4.4. This may contribute to anomalous heat loss trom a plasma. 


\section{REFERENCES FOR CHAPTER V}

Rosenbluth, M. N., Los Alamos Scientific Research Laboratory Report LA-2030 (1956).

Parker, G. N., Dynamic instability of an anisotropic ionized gas of low density, Phys, Rev. 109, 1874 (1958).

Roesenbluth, M. N., and Rutherford, P. H., Excitation of Alfvén waves by high energy ions in a Tokamak, Phys. Rev. Lett. , 34, 1428 (1975).

S., Hydrodynamic and hydromagnetic stability, Chapter 13, Oxford, Clarendon, 1961.

Mikhailovskii, Review of Plasma Physics, ed. by M. A. Leontovich, p. 172, New York, Consultant Bureau (1967). Tsang, K. T., Whitson, J. C.,Callen, J. D., Smith, J., Drift Alfvén waves in Tokamaks, Phys. Rev. Lett. $\underline{41}, 557$ $(1978)$ 


\section{OTHER \\ REFERENCES FOR CHAPTER V}

Benezin, Yu. A., Vshivkov, V. A., On the firehose instability of Alfvén waves, J. Computer Phys. 20, 8196 (1976). Bizli, K. O., Lominadze, D. G., Mikhailovskii, A. G., Stimulation of shortwave Alfvén waves by high-energy ions in Tokamak, Fiz. Plasmy 2 , (1976).

Chao-Kung Yang, Sunnerup, B. U. O., Compressive Magnetic field reconnection a slow wave model, Astrophys. J. 206, 570-82 (1976).

Dobrowolny, M. Kelvin-Helmholtz Instability in a high-beta collisionless plasma, Phys. Fluids 15, 2263-70 (1972). Egorenkov, V. D., Stepanov, K. N., Electromagnetic instabilities in a finite-beta plasma with hot electrons, zh. Tekh. Fiz. 43, 270-5 (1973), Sov. Phys.-Tech. Phys. $\underline{18}$ 178-80 (1973).

Egorenkov, V. D., Low-frequency instabilities of cold plasma with a group of hot ions with anisotropic velocity distribution function, Ikr. Fiz. Zh. 18, 1472-5 (1973): Elsasser, K., Schamel, H., Nonlinear evolution of firehoseunstable Alfvén waves, J. Plasma Phys. ㄱ, 475-502 (1972). Hasegawa, A., Plasma Instabilities and Nonlinear Effects, New York, Springer-Verlag, 1975. Hudson, M. K., Kennel, C. F., The electromagnetic interchange mode in a partly-ionized collisional plasma, J. Plasma Phys. 14, 121-34 (1975). 


\section{OTHER}

REFERENCES FOR CHAPTER V

Kaladze, T. D., Lominadze, D. G., Stepanov, K. N., Excitation of Alfvén waves by fast ions in a finitebeta plasma, Zh. Tekh. Fiz. 43, 2517-20 (1973), Sov. Phys.-Tech. Phys. 18, 1583-4 (1974).

Kan, J. R. Heacock, R. R., Generation of irregular (type PI C) pulsations in the plasma sheet during substorms, J. Geophys. Res. $81,2371-7$ (1976).

Kats, M. E., Makarenko, V. N., Yukhimuk, A. K., E. M. Wave instability in plasma with a 'reverse loss cone' (application to magnetosphere), Zh. Tekh. Fiz. 2, 133-7 (1976). Landau, R. W., Cuperman, S., Stability of anisotropic plasmas to almost-perpendicular magnetosonic waves, J. Plasma Phys. 6 , 495-512 (1971).

Mazur, V. A., Mikhailovskii, A. B., Excitation of Alfvén waves by fast ions in Tokamak with non-circular crosssection, Fiz. Plazmy 2, 172-5 (1976).

Mikhailovskii, A. B., Pokhote'ov, O. A., A new mechanism of generation of geomagnetic pulsations by fast particles, Fiz. Plazmy 1, 786-92 (1975).

Mikhailovskii, A. B., 'Drift' stimulation of Alfvén waves by trapped electrons in Tokamak at beta/sub J/\#l, Fiz. Plazmy 1, 378-92 (1975).

Mikhailovskii, A. B., 'Drift' instabilities distorting the magnetic surfaces of Tokamak-type Toroidal systems, Nucl. Fusion 13, 259-69 (1973). 


\section{OTHER}

\section{REFERENCES FOR CHAPTER V}

Montgomery, M. D., Gary, S. O., Fieldman, W. C., Forslund, D. W., Electromagnetic instabilities driven by unequal proton beams in the solar wind, J. Geophys, Res. 81 , 2743-9 (1976).

Nishida, Y., Ishii, K., Observation of the coupled mode of a collisional drift wave and an Alfvén wave, Phys. Rev. Lett. $33,352-5$ (1974).

Ohsawa, Y., Nosaki, K., Hasegawa, A., Kinetic theory of magnetohydrodynamic Kelvin-Helmholtz instability, Phys. Fluids 19, 1139-43 (1976).

Roberts, B., Overstability and cooling in sunspots, Astrophys. J. $204,268-80(1976)$.

Tang, J. T., Luhmann, N. G., Jr., Nishida, Y., Ishii, K., Destabilization of hydromagnetic arift-Alfvén waves in a finite-beta collisional plasma, Phys. Rev. Lett. $\underline{34}, 70-3(1975)$. 
VI. NONLINEAR PROCESSES

\subsection{Introduction}

In the chapters up to now we have considered small amplitude Alfven waves. When the perturbed magnetic field is no longer small compared to the ambient magnetic field the study of nonlinear MHD equation becomes necessary. It is now well known that a circularly polarized Alfvén wave described by $\underset{\sim}{B}=\left(B_{0}, B_{y}, B_{z}\right)$ and $\underset{\sim}{v}=\left(0, v_{y}, v_{z}\right)$ is an exact solution of the nonlinear magnetohydrodynamic equations (Ferraro, 1956). Ferraxo aiso pointed out the equipartition of kinetic and mechanical energy, characteristic of the magnetohydrodynamic waves. Can large amplitude Alfvên wave persist in a real plasma? The decay instability of this wave into a small amplitude Alfvén wave and a sound wave has been discussed in detail by Sagdeev and Galeev (1969).

The large amplitude linearly polarized Alfvén wave on the other hand, has the local phase velocity

$$
v_{p h}^{2}=\left[B_{0}^{2}+B(t)^{2}\right] / \mu_{0} \rho
$$

which, can be seen to approach the Alfvén velocity as $B(t) / B_{0} \rightarrow 0$. The rapid development of plane polarized Alfvén wave into hydromagnetic shock has been discussed by Montgomery (1958) and was observed experimentally by Boley and Forman (1964). 
The possibility of the propagation of solitary hydromagnetic waves in the direction parallel to a uniform magnetic field in a cold collision-free plasma was shown by shaffman (1961). It was noted that solitary Alfvén waves move much faster the linearized Alfvén waves in the plasma.

A large number of papers on the nonlinear effects of the MHD Alfvén waves are seen in the comparatively recent literature. However, as we have seen in the linear theory of the Alfvén waves, in most laboratory scale plasmas, its inhomogeneity prohibits the existence of the ideal MHD Alfvén wave. Hence the nonlinear theory of MHD Alfvén wave is not applicable in most laboratory plasmas. Hence, in this chapter we shall consider nonlinear processes associated with the Alfvên wave perturbation with particular emphasis on its kinetic effects.

We discuss quasi-linear diffusion in the presence of the kinetic Alfvén wave turbulence, section 6.2 parametric decay and nonlinear Landau damping of the kinetic Alfvén, Section 6.3, some solitary wave solutions, section 6.4, and the modulational instability of the finite frequency Alfvén wave, Section 6.5 .

\subsection{Quasi-Linear Diffusion}

In the collisionless plasma with a stationary ensemble of waves, the quasi-linear diffusion occurs only in the presence of wave-particle resonant interactions. The MHD Alfvén wave does not have the wave-particle resonant interactions, hence there should be no quasi-linear diffusion; one should introduce either a finite frequency or finite wave number effects. 
Consequently, we consider here the quasi-linear diffusion in the presence of the kinetic Al fvén wave turbulence (Hasegawa and Mima, 1978). This is relevant in fusion plasma because the kinetic Alfvén wave is excited for example by the drift wave instability.

We first consider the electron diffusion. We use the drift kinetic equation (Appendix), for the electron guiding, center distribution function $f_{e}\left(v_{\|}, \underset{\sim}{x}, t\right)$.

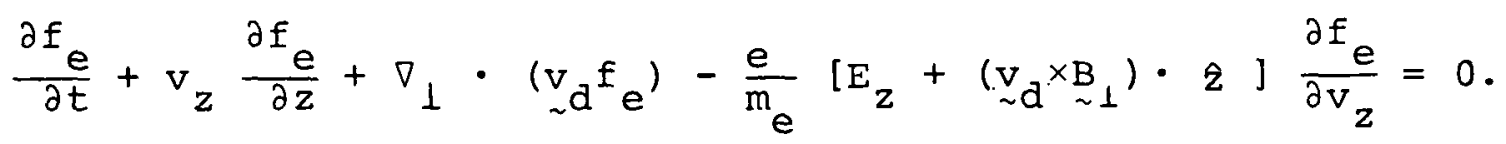

Here we used a local Cartesian coordinate in which the $z$ axis is taken in the direction of the ambient magnetic field $\underset{\sim}{\mathrm{B}} 0^{\prime} \underset{\sim}{\mathrm{E}}$ and $\underset{\sim}{\mathrm{B}}$ are the wave electric and magnetic fields with subscripts $z$ and $\perp$ indicating the components in the direction parallcl and perpendicular to the ambient magnetic field respectively. $\quad{\underset{\sim}{\mathrm{v}}}_{\mathrm{d}}$ is the drift velocity perpendicular to the magnetic field which, for electrons, is given by

$$
{\underset{\sim}{\mathrm{d}}}_{\mathrm{d}}=\frac{\stackrel{\mathrm{E}_{1} \times \mathrm{B}_{0}}{\sim}}{\mathrm{B}_{0}^{2}}+\frac{\mathrm{B}_{1}}{\mathrm{~B}_{0}} \mathrm{v}_{\mathrm{z}}
$$

We assumed also that the plasma $B$ is small (compared with unity) to warrant neglection of the magnetic compression $\mathrm{B}_{\mathbf{z}}$. 
We assume that the density gradient exists in the $\mathrm{x}$ direction. Then the quasi-linear equation for the average distribution function $\left\langle\mathrm{f} \mathrm{e}^{\rangle}\right.$can be written as

$$
\frac{\partial\left\langle f_{e}\right\rangle}{\partial t}+\left\langle\frac{\partial}{\partial x} \frac{1}{B_{0}}\left(E_{y}+v_{z} B_{x}\right) f_{e}^{(1)}\right\rangle-\frac{e}{m_{e}}\left\langle E_{z} \frac{\partial f_{e}^{(1)}}{\partial v_{z}}>=0,\right.
$$

where $\mathrm{f}_{\mathrm{e}}^{(1)}$ is the linear response and <> shows the ensemble average. If we use the Fourier amplitude expression for the linear terms, such as $\mathrm{f}_{\mathrm{e}}^{(1)}=$ $\operatorname{Re} \sum_{\sim} f_{\underset{\sim}{k}}^{(1)}(x) e^{i\left(\underset{\sim}{k} \cdot \underset{\sim}{x}-\omega_{t}\right)}$, the linear response of the distribution function ${\underset{\sim}{\mathrm{k}}}_{\sim}^{(1)}$ can be expressed as

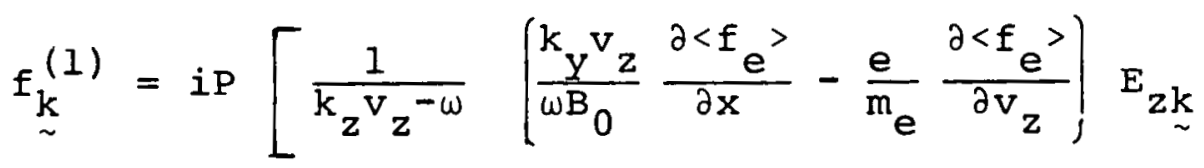

$$
\begin{aligned}
& \left.-\frac{1}{\omega B_{0}} \frac{\partial<\bar{f} e^{>}}{\partial x} E_{y k}\right] \\
& +\pi \delta\left(k_{z} v_{z}-\omega\right)\left(\frac{e}{m_{e}} \frac{\partial<f_{e}>}{\partial v_{z}}-\frac{k_{y} v_{z}}{\omega B_{0}} \frac{\partial<f_{e}>}{\partial x}\right) E_{z \underset{\sim}{k}},
\end{aligned}
$$

where $P$ indicates the principal value, $\delta$ the Dirac's $\delta$ function, and the Maxwell's equation $\nabla \times \underset{\sim}{E}=-\partial \underset{\sim}{B} / \partial t$ is used to cxprese $B_{x}$ in terms nf $F_{i y}$ and $E_{z}$; 


$$
\mathrm{B}_{\mathrm{xk}}=\left(\mathrm{\sim}_{\mathrm{\sim}} \mathrm{E}_{\mathrm{z}}^{\mathrm{\sim}} \underset{\sim}{\mathrm{\sim}}-\mathrm{k}_{\mathrm{z}}^{\mathrm{E}} \underset{\sim}{\mathrm{\sim}}\right) / \omega
$$

-If we substitute Eq. (6.4) into (6.3), the term multiplied by the delta function, which represents the dissipation due to the Landau damping, contributes to the quasi-linear diffusion coefficient. The first term contributes to the diffusion in velocity space, and the second term to that in coordinate space. Taking the second term contribution here we have

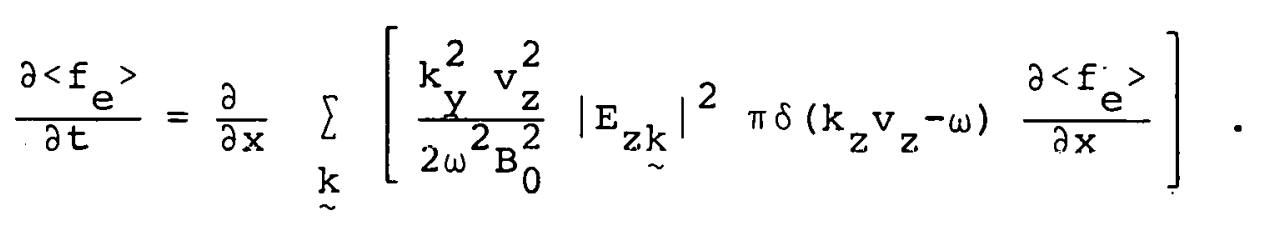

As is expected only the $z$ component of the wave electric field contributes to the diffusion. The above result is valid for any kind of low frequency electromagnetic waves not restricted to the kinetic Alfven wave.

The diffusion equation for electron density $\mathrm{n}_{\mathrm{e}}=\int\left\langle\mathrm{f}_{\mathrm{e}} \mathrm{ddv}_{\mathrm{z}}\right.$ can be constructed by integrating Eq. (6.6) over $v_{z}$. If we assume a Gaussian distribution for $\left\langle\mathrm{f} e{ }^{\rangle}\right.$, i.e.,

$$
\left\langle f_{e}\right\rangle=n_{e}(x) \frac{1}{\sqrt{2 \pi} v_{T e}} e^{-v_{z}^{2} / 2 v_{T e}^{2}}
$$


we have, for $\mathrm{v}_{\mathrm{Te}}>\mathrm{v}_{\mathrm{A}}$ '

$$
\left.\frac{\partial n_{e}}{\partial t}=\frac{\partial}{\partial x} \quad D_{e} \frac{\partial n_{e}}{\partial x}\right)
$$

where

$$
D_{e}=\sqrt{\frac{\pi}{8}} \sum_{\substack{k \\ \sum}}^{k_{y}^{2}\left|E_{z k}\right|^{2}} \frac{1}{k_{z}^{2} B_{0}^{2}} \frac{1}{\left|k_{z}\right| v_{T e}} .
$$

As was stated previously, a unique feature of the kinetic Alfvén wave is that it accompanies the electric field $E_{z}$ in the direction of the ambient magnetic field.

We now discuss the ion diffusion. The diffusion coefficient can be formally obtained by using $f_{i}$ in place of $f_{e}$ in Eq. $(6.6)$. However because $f_{i}\left(v=\omega / k_{z}=v_{A}\right)$. $\exp \left(-v_{A}^{2} / 2 v_{T i}^{2}\right) \sim \exp \left(-\beta^{-1}\right)$, if the plasma $\beta$ is small the diffusion coefficient obtained this way gives a negligible small value compared with that of electrons.

However, it can be shown that if a local energy balance is maintained between electron and ion dýnamics, the ambipolarity results, namely the ion diffusion coefficient becomes identical to that of the electrons. To present an example of such a case we consider a situation in which the fluctuation is generated by the drift wave instability. When the local energy balance exists, the fluctuation excited by the inverse Landau damping of electrons is absorbed by 
ion damping and stationally state is maintained. If we take the viscous damping as the damping mechanism by the ions, and ignore the finite ion Larmour radius effect for simplicity, the ion density fluctuation $\mathrm{n}_{i k}$ is obtained from the equation of motion and the equation of continuity,

$$
\mathrm{n}_{i \underset{\sim}{ }}=\frac{\mathrm{n}_{0}}{i \omega}\left[\frac{1}{\mathrm{n}_{0}} \frac{\partial \mathrm{n}_{0}}{\partial \mathrm{x}} \frac{\mathrm{E}_{\mathrm{yk}}}{\overline{\mathrm{B}}_{0}}+\frac{k_{y} \omega\left(1+i \nu k_{y}^{2} / \omega\right)}{\omega_{C i}} \frac{\mathrm{E}_{\mathrm{yk}}}{\mathrm{B}_{0}}\right] \text {, }
$$

where $\nu$ is the viscosity coefficient, and the parallel ion inertia is ignored for the Alfvén branch considered here. We note that the diffusion coefficient $D_{i}$ is obtained by calculating the diffusive ion flux,

$$
\begin{aligned}
J_{D i} & =\left\langle n_{i \underset{\sim}{k}}^{v} v_{\sim \sim}^{k}\right\rangle \\
& =\frac{1}{2} \operatorname{Re}\left[\underset{\sim}{n_{\sim}^{k} E_{y k}^{k}}\right] \\
& \equiv-D_{i} \frac{\partial n_{0}}{\partial x} .
\end{aligned}
$$

If we substitute Eq. (6.10) to this expression, we can formally obtain the ion flux in terms of the viscosity coefficient $v$,

$$
J_{D i}=\frac{1}{2} \frac{\left|E_{y \underset{\sim}{j}}^{\prime}\right|^{2}}{B_{0}^{2}} \frac{k_{y}^{3} v n_{0}}{\omega \omega_{C i}} .
$$

To compare this with the electron diffusive flux, Eq. (6.8), we need to exprese $\nu$ and $E_{y k}$ in terms of $\delta_{e}$ and $E_{z k}$. For For this purpose we derive the dispersion relation using 
quasi-neutrality and Ampere's law including the effect of the density gradient. By assuming stationary state (real

w), we have, from the real part of the dispersion relation, Eq. (5.22),

$$
\left(\omega-\omega^{*}\right)\left(\omega^{2}-k_{z}^{2} v_{A}^{2}\right)=\lambda_{s} k_{z}^{2} v_{A}^{2} \omega,
$$

and from the imaginary part

$$
\delta_{e^{\omega}}=\nu k_{y}^{2} \frac{\omega * / \omega-1}{\lambda},
$$

where $\omega^{*}$ is the drift wave frequency given by

$$
\omega^{*}=\frac{k_{y} v_{T e}^{2}}{\omega_{c e}} \frac{1}{n_{0}} \frac{\partial n_{0}}{\partial x}
$$

The relation between $\mathrm{E}_{z \underset{\sim}{k}}$ and $\mathrm{E}_{\mathbf{y}_{\sim}}$ may be obtained from Ampere's law;

$$
E_{\underset{\sim}{k}}=\frac{k}{k_{z}} E_{z \underset{\sim}{k}}\left[1-\frac{\omega(\omega-\omega *)}{\lambda_{s} k_{z}^{2} v_{A}^{2}}\right]
$$

Substituting Eqs. (6.14) and (6.15) and using the dispersion relation (6.13) into $(6.12)$, we have

$$
J_{D i}=\frac{1}{2}\left(\frac{k y}{k_{z}}\right)^{-2} \frac{\left|E_{z k}\right|^{2}}{B_{0}^{2}}\left(\frac{\omega^{*}}{\omega}-1\right) \frac{k_{y} n_{0}}{\omega_{c i}} \frac{\delta_{e}}{\lambda_{s}} .
$$

If we compare this expression with Eq. (6.11), we see 


$$
D_{i}=\sqrt{\frac{\pi}{8}}{\underset{\sim}{k}}_{\underset{z}{\sum} \frac{k_{y}^{2}\left|E_{z k}\right|^{2}}{k_{z}^{2} B_{0}^{2}}} \frac{1}{\left|k_{z}\right| v_{T e}}=D_{e},
$$

hence the ambipolarity is proved.

However, we point out that if the local energy balance between the electron and ion dynamics does not exist, the ambipolarity disappears. For example if the stationary state is achieved whereby the fluctuation produced by the electrons are convected away by the wave, or reabsorbed by electrons, the ion diffusion coefficient becomes pratically zero. 


\subsection{Parametric Decay}

The MHD Alfvén wave is known to decay into another

Mlfvên wave and the ion acoustic wave (Sagdecv anci Gaieev, 1969). Hence we treat the decay of the kinetic Alfvén wave here, which has a much larger decay rate than the MHD Alfvén wave (Hasegawa and Chan, 1976). We consider a selfconsistent $\mathrm{pump}$ wave $\phi_{0}(\underset{\sim}{x}, t)$ (the kinetic Al fvén wave) of the following form

$$
\phi_{0}(\underset{\sim}{x}, t)=\frac{1}{2}\left\{\phi_{0} \exp \left[-i\left(\omega_{0} t-\mathrm{x}_{0} \underset{\sim}{x}\right)\right]+c \cdot c\right\} .
$$

Here, $\left(\omega_{0}, k_{0}\right)$ satisfies the linear dispersion relation for kinetic Alfvén wave. The pump field $\phi_{0}$ is assumed to be sufficiently weak so that only interactions up to $0\left(\left|\phi_{0}\right|^{2}\right)$ need to be kept. Furthermore, since decay instabilities are considered here, we ignore the upper sideband as being offresonant and only discuss the couplings among the pump wave. $\left( \pm \omega_{0}, \underset{\sim}{ \pm k_{0}}\right)$, the lower sideband $\left(\omega_{-}, \underset{\sim}{k_{-}}\right)=\left(\omega_{s}-\omega_{0},{\underset{\sim}{s}}_{\mathbf{s}_{0}}^{-k_{0}}\right)$ and the low frequency wave $\left(\omega_{s},{\underset{\sim}{s}}_{s}\right)$. Note, again $\left( \pm \omega_{0}, \pm k_{0}\right)$ and $\left(\omega_{-}, k_{-}\right)$are kinetic Alfvén waves, and $\left(\omega_{s}, k_{s}\right)$ is the ion acoustic mode. For low $B$ plasmas, $\left|\omega_{0}\right| \sim\left|k_{z} v_{A}\right|>\left|\omega_{S}\right|$ $\sim\left|k_{z} c_{s}\right|$. To further simplify the analyses, we make the additional assumptions that $\left|\mathrm{k}_{1} \rho_{i}\right|<1$ and $\mathrm{T}_{\mathrm{e}} / \mathrm{T}_{i}>1$, so that we ignore the finite ion Larmour radius effect. The dynamics of both species are then described by the following drift kinetic equation 


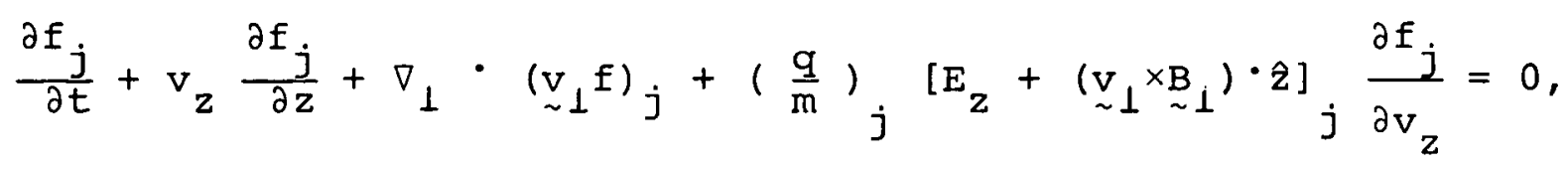

where

$$
\underset{\sim}{v} \perp_{j}=\left({\underset{\sim E}{E}}_{\sim P}+v_{\sim B}+v_{B}\right)_{j}, j=e, i
$$

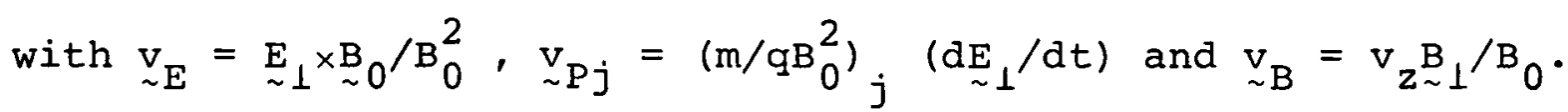
$f_{j}\left(x_{\sim}, v_{z}, t\right)$ is the drift distribution function and other notations are standard. Note here, $\frac{d}{d t}$ contains a convective term; i.e., $\frac{d}{d t}=\frac{\partial}{\partial t}+\underset{\sim}{(\underset{\sim}{\nabla})}$ ).

$$
\text { Let } f_{j}=f_{j}^{(0)}+f_{j}^{(1)}+f_{j}^{N L} \text { and using the two }
$$

potentials $\phi$ and $\psi$ defined in Eqs. (2.7) and (2.8), we obtain the linear response $\mathrm{f}_{j}^{(1)}$ as

$$
f_{e}^{(1)}=f_{e}^{(0)} \bar{\psi},
$$

and

$$
f_{i}^{(1)}=-\bar{\lambda} f_{i}^{(0)} \phi+c_{s}^{2} \frac{\partial f_{i}^{(0)} / \partial v_{z}}{v_{z}-\omega / k} \bar{\psi},
$$

where $\bar{\psi}=\mathrm{e} \psi / \mathrm{T}_{\mathrm{e}^{\prime}}, \bar{\phi}=\mathrm{e} \phi / \mathrm{T}_{\mathrm{e}}$ and $\bar{\lambda}=\mathrm{k}_{1}^{2} \mathrm{c}_{\mathrm{s}}^{2} / \omega_{\mathrm{ci}}^{2}$. Equations (6.20) and (6.21) are valid for both kinetic Alfvén and ion acoustic waves. Note, however, since $\left(\omega_{s}, \underset{\sim}{k}\right)$ is an 
electrostatic mode, $\phi_{S}=\psi_{s}$; while for the kinetic Alfvén modes, $\left(\omega_{-},{\underset{\sim}{k}}_{-}\right)$and $\left(\omega_{0},{\underset{\sim}{0}}_{0}\right), \phi_{-, 0} \neq \psi_{-, 0}$.

In dealing with the nonlinear analyses, let us note that because the kinetic Alfvén waves have $\bar{\lambda} \sim O(1)$, our results are, therefore, valid in the regime $\bar{\lambda} \gg \omega_{0} / \omega_{\mathrm{ci}}$; while the classic magnetohydrodynamic results of sagdeev and Galeev (1969) are valid in the opposite limit. The details of nonlinear interactions are different for the different modes as well as species and will be treated separately.

Let us first consider the ion acoustic $\left(\omega_{s^{\prime}}{ }_{\sim}^{k}\right)$ mode. Since we are interested in either the resonant decay to the ion acoustic mode or the induced scattering decay when this mode is heavily damped, only nonlinear perturbations up to $0\left(\left|\phi_{0}\right|\right)$ need to be kept here; i.e., we only have to calculate $f_{j}^{(2)}$. For the electrons $\left|\omega_{s}\right|,\left|\omega_{0}\right| \ll \omega_{c e}$ and $\left|k_{1} \rho_{e}\right| \ll\left|k_{\perp} \rho_{i}\right|<1, v_{\sim p e}$, the electron polarization drift, thus, can be neglected. The dominant nonlinear contributions to $\mathrm{f}_{\mathrm{e}}^{(2)}$ is then found to originate from, referring to Eq. (6.18), the $\underset{\sim}{\nabla} \cdot\left[\underset{\sim}{v_{B}} \underset{\sim}{\left(\Omega_{0}\right) f_{e}^{(1)}}\left(\underset{\sim}{\left.\Omega_{-}\right)}+(0+-)\right]\right.$ term as well as the

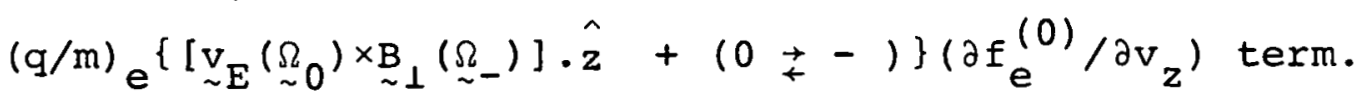

Here, we have denoted $\Omega_{\sim} \equiv\left(\omega_{0},{\underset{\sim}{0}}_{0}\right),{\underset{\sim}{0}}_{0} \equiv\left(\omega_{-}, \underset{\sim}{\mathrm{k}}\right)$ and $\stackrel{\sim}{s}_{s} \equiv\left(\omega_{s}{ }_{\sim s}^{k}\right)$. With $\left|\omega_{s}\right| \ll\left|k_{z_{s}} v_{T e}\right|, f_{e}^{(2)}\left(\underset{\sim s}{\left.\Omega_{s}\right)}\right.$ is given by 


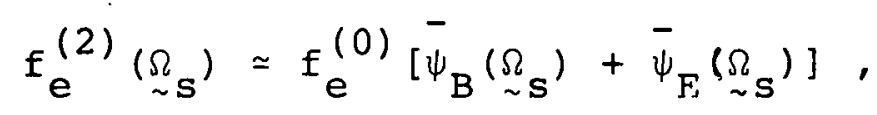

where $\bar{\psi}_{B}$ corresponds to the $\underset{\sim}{\mathrm{B}_{\mathrm{B}}} \mathrm{f}_{\mathrm{e}}^{(1)}$ term;

$$
\begin{aligned}
\bar{\psi}_{B}\left(\Omega_{\sim S}\right)=\frac{i c_{s}^{2}}{2 \omega_{C i} k_{\| S}} & \left({\underset{\sim}{s}}_{S} \times k_{\sim}\right) \cdot \hat{z}\left[\frac{k_{\| 0} \bar{\lambda}_{-}\left(1+\lambda_{0}\right)}{\omega_{0}}\right. \\
& \left.-\frac{k_{\|-} \bar{\lambda}_{0}\left(1+\bar{\lambda}_{-}\right)}{\omega_{-}}\right] \bar{\phi}_{-} \bar{\phi}_{0} .
\end{aligned}
$$

and $\bar{\psi}_{E}$ corresponds to the $\underset{\sim E}{\underset{E}{v}} \times \underset{\sim}{B}$ term;

$$
\begin{aligned}
\bar{\psi}_{E}\left(\Omega_{\sim}\right)=-\frac{i c_{S}^{2}}{2 \omega_{C i} k_{\| S}} & \left({\underset{\sim}{s}}_{s} \times{\underset{\sim}{k}}_{0}\right) \cdot \hat{z}\left[\frac{k_{\| 0}\left(1+\bar{\lambda}_{0}\right)}{\omega_{0}}\right. \\
& \left.-\frac{k_{\|-\left(1+\bar{\lambda}_{-}\right)}}{\omega_{-}}\right] \phi_{-} \phi_{0} .
\end{aligned}
$$

In deriving Eqs. (6.23) and (6.24), we have made use of the fact that $\Omega_{0}$ and $\Omega_{\sim}$ are kinetic Alfvên waves and, hence, $\psi\left(\operatorname{or} E_{z}\right)$ and $\underset{\sim}{B}$ are related to $\phi$ by $E q .(6.5)$.

In treating the ions, we note first that because $\left|\omega_{-}\right|,\left|\omega_{0}\right| \gg\left|k_{\| 0} v_{T i}\right|,\left|k_{\|}-v_{T i}\right|, v_{\sim B}$ has negligible contribution and, from Eq. (6.21), $f_{i}^{(1)}$ of the kinetic Alfvén wave is reduced to

$$
\begin{aligned}
& f_{i}^{(1)} \underset{\sim}{(\Omega)}=-\bar{\lambda} f_{i}^{(0)} \bar{\phi} \underset{\sim}{(\Omega)}+\frac{k_{z} v z}{\omega} f_{i}^{(0)} \frac{{ }^{T} e}{T_{i}} \psi(\underset{\sim}{(\Omega)} \\
& \equiv f_{i l}^{(1)}+f_{i z}^{(1)}, \text { for } \underset{\sim}{\Omega_{2}}=\underset{\sim}{\Omega_{0}}, \underset{\sim}{\Omega} \Omega_{-} .
\end{aligned}
$$


Secondly, we note that $\underset{\sim p i}{V}$ contains a nonlinear term from

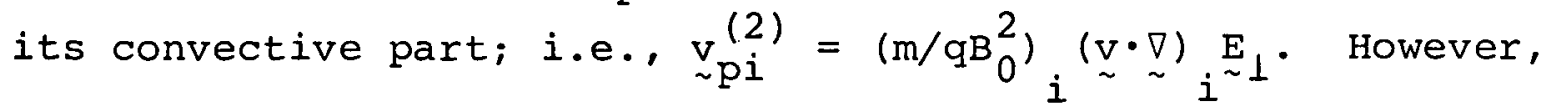
it can be shown that the contribution to the $\underset{\sim}{\nabla} \cdot\left[{\underset{\sim}{1}}_{1} f\right]$ term from $\underset{\sim p i}{(2)} f_{i \perp}^{(0)}$ cancels to the order $\omega_{0} / \omega_{c i}$ with that from ${\underset{\sim E}{E}}_{i \perp}^{(1)}$. Thus, the only net contribution comes from the

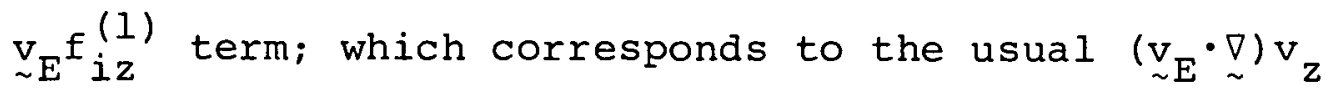
convective nonlinear (ponderomotive) force term in the parallel (to $\underset{\sim}{\mathrm{B}} 0$ ) equation of motion. Another important nonlinear contribution comes from the $\left(\underset{\sim E}{\underset{\sim}{v} \times 1}{ }_{\sim}^{B}\right) \cdot \hat{z}$ term, similar to that of the electrons. Combining these nonlinear terms, we obtain

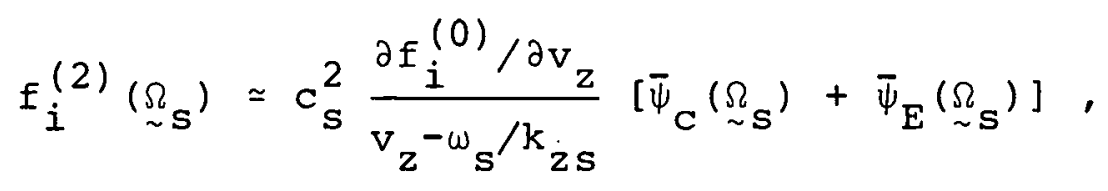

where $\Psi_{C}$ corresponds to the $\underset{\sim}{\nabla} \cdot\left[{\underset{\sim}{E}}_{E}^{f}(1)\right]$ nonlinearity and is given by

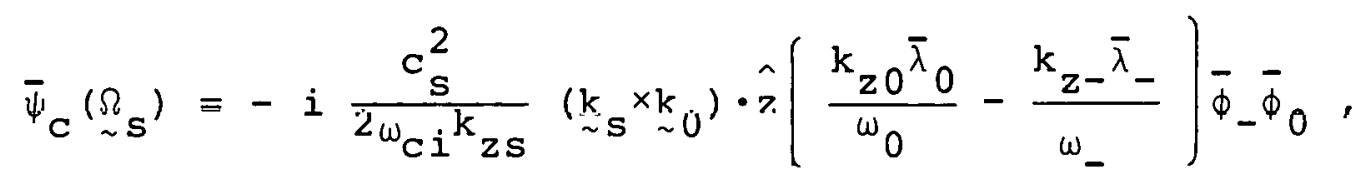

and $\bar{\psi}_{E}\left(\Omega_{S}\right)$ is given in Eq. (6.24). Substituting Eqs. $(6.20)$, $(6.21),(6.22)$, and $(6.26)$ into the quasi-neutrality equation

$$
\left[n_{e}^{(1)}\left(\Omega_{s}\right)+n_{e}^{(2)}\left(\Omega_{s}\right)\right]=\left[n_{i}^{(1)} \underset{\sim s}{\left(\Omega_{s}\right)+n_{i}^{(2)}}\left(\Omega_{s}\right)\right],
$$

we have 


$$
\varepsilon_{\mathbf{s}}\left(\Omega_{\sim}\right) \bar{\phi}_{s}=\Lambda_{s}\left[F(\bar{\lambda})+\varepsilon_{s}\left(\Omega_{\sim}\right)\right] \bar{\phi}_{0} \bar{\phi}_{-},
$$

where

$$
\begin{gathered}
\varepsilon_{s}=1+\bar{\lambda}_{s}+x_{i}, \\
x_{i}=-c_{s}^{2} \int \frac{\partial f_{i}^{(0)} / \partial v_{z}}{v_{z}-\omega_{s} / k_{z s}} d v_{z}, \\
\Lambda_{s}=\frac{\Lambda}{k_{z s}}\left(\frac{k_{z 0}}{\omega_{0}}-\frac{k_{z-}}{\omega_{-}}\right) \simeq \frac{\Lambda}{\omega_{0}}, \\
\Lambda=-i c_{s}^{2} \frac{\sim_{\sim s}^{\left(k_{s} \times k_{0}\right) \cdot \hat{z}}}{2 \omega_{c i}},
\end{gathered}
$$

and

$$
F(\bar{\lambda})=\bar{\lambda}_{0}+\bar{\lambda}_{-}+\bar{\lambda}_{0} \bar{\lambda}_{-}-\bar{\lambda}_{s} \cdot
$$

Equation (6.28) describes the coupling of the ion acoustic mode to the lower-sideband kinetic Alfven wave via the pump. Let us now consider the kinetic Alfvén $\left(\omega_{-},{\underset{\sim}{-}}_{-}\right)$ mode. For this mode we have to calculate both the charge and parallel current density perturbations to $0\left(\left|\bar{\phi}_{0}\right|^{2}\right)$; i.e., $f_{j}^{(3)}\left(\Omega_{\sim}\right)$ in order to take into account the induced scattering process. 
Thus, nonlinear contributions due to both $f_{j}^{(1)}\left(\Omega_{\sim}\right)$ and $f_{j}^{(2)}\left(\underset{\sim}{\Omega_{S}}\right)$ must be included. Let us first consider the electrons. Again, $\underset{\sim p}{v}$ pe has negligible contribution and dominant nonlinear contributions come from the $v_{\sim}^{*}\left(\Omega_{\sim}\right)\left[f(1)\left(\Omega_{s}\right)+f_{e}^{(2)}\left(\Omega_{\sim}\right)\right]$ term as well as the

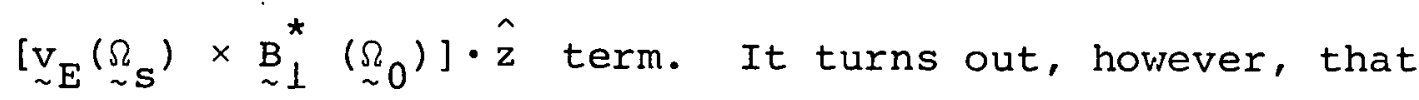
$\mathrm{n}_{e}^{(2)}$ is negligible compared to $\mathrm{n}_{i}^{(2)}$ due to the canceling of dominant nonlinear terms; while $n_{e}^{(3)}$ is given by

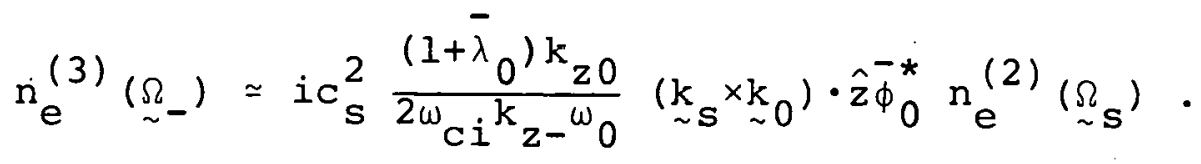

Perturbations in parallel current density can then be obtained from the continuity equation;

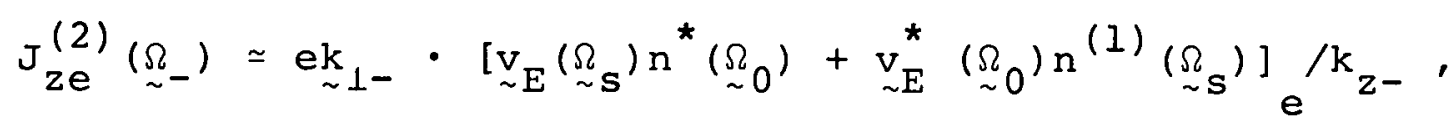

and

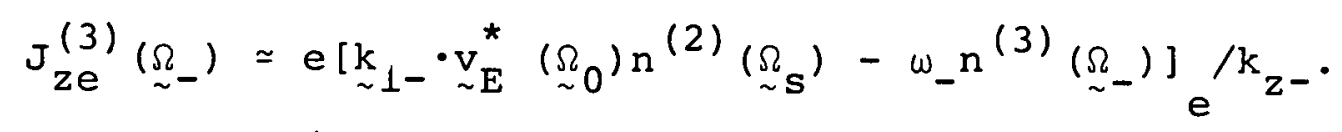

As to the ions, because $\left|\omega_{-}\right| \gg\left|\mathrm{k}_{z_{-}} \mathrm{v}_{\mathrm{Ti}}\right|$, the dynamic is mainly in the perpendicular (to $\underset{\sim}{\mathrm{B}}$ ) plane. 
Thus, we can neglect $J_{z i}$ and, from the continuity equation, we find the ion density perturbations to be

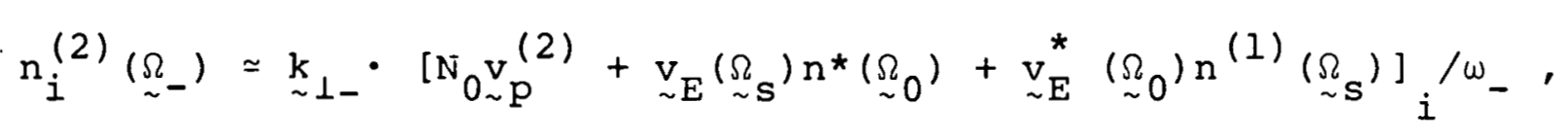

and

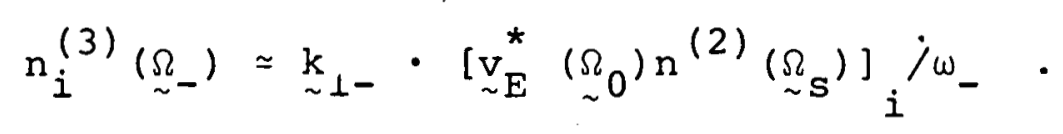

Here, $v_{\mathrm{pi}}^{(2)}\left(\Omega_{\sim}\right)$ is the nonlinear ion polarization drift due to the convective $\left({\underset{\sim}{v}}_{1} \stackrel{\sim}{\sim}_{1}\right)$ term.

The two field equations, Eqs. (2.9) and (2.10),

including nonlinear perturbations become at $\underset{\sim}{\Omega}=\Omega_{\sim}-$

$$
\begin{aligned}
& n_{e}^{(1)}+n_{e}^{(2)}+n_{e}^{(3)}=n_{i}^{(1)}+n_{i}^{(2)}+n_{i}^{(3)} . \\
& \frac{\partial}{\partial z} \nabla_{\perp}^{2}(\phi-\psi)=\mu_{0} \underset{j=e, i}{\sum} \frac{\partial}{\partial t}\left(J_{z j}^{(1)}+J_{z j}^{(2)}+J_{z j}^{(3)}\right),
\end{aligned}
$$

which can be produced to a single equation,

$$
\begin{aligned}
\varepsilon_{\mathrm{A}}\left(\Omega_{\sim}\right) \bar{\phi}_{-}= & \frac{1}{\mathrm{~N}_{0} \omega_{-} \bar{\lambda}_{-}\left(1+\bar{\lambda}_{-}\right)}\left[\omega_{-}\left(\mathrm{n}_{-}^{(2)}+\mathrm{n}_{-}^{(3)}\right)\right. \\
& \left.-\left(1+\bar{\lambda}_{-}\right) \mathrm{k}_{\mathrm{z}-}\left(\mathrm{J}_{z-}^{(2)}+\mathrm{J}_{z-}^{(3)}\right) / \mathrm{e}\right],
\end{aligned}
$$


where

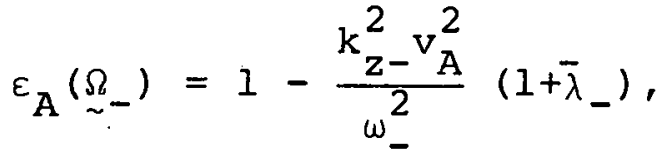

$$
\begin{aligned}
& \mathrm{n}_{-}^{(2),(3)}=\left[\mathrm{n}_{\mathrm{i}}\left({\underset{\sim}{-}}_{-}\right)-\mathrm{n}_{\mathrm{e}}{\left.\underset{\sim}{\left(\Omega_{-}\right.}\right)}^{(2),(3)},\right.
\end{aligned}
$$

and

$$
\mathrm{J}_{\mathrm{z}-}^{(2),(3)} \simeq \mathrm{J}_{\mathrm{ze}}^{(2),(3)} \underset{\sim-}{\left(\Omega_{-}\right)}
$$

In deriving Eq. (6.41), we have noted that $\Omega_{-}$is a resonant kinetic Alfvén mode and, hence, $\left|\varepsilon_{A}\right|<1$. Substituting Eq. (6.34) to (6.38) for the nonlinear charge density and parallel current density perturbations into Eq. (6.41), it reduces to

$$
\tilde{\varepsilon}_{A}\left(\Omega_{\sim}\right) \bar{\phi}_{-}=\Lambda_{A} F(\bar{\lambda}) \bar{\phi}_{0}^{*} \bar{\phi}_{S},
$$

where

$$
\tilde{\varepsilon}_{A}=\varepsilon_{A}-\varepsilon_{A}^{(3)},
$$

$\varepsilon_{A}^{(3)}$ is due to third order perturbations

$$
\begin{aligned}
\varepsilon_{A}^{(3)=}\left|\bar{\phi}_{0}\right|^{2}|\Lambda|^{2} \frac{\left(1+\bar{\lambda}_{0}\right)}{k_{z S}{ }^{\omega}-} & {\left[1-\frac{k_{z 0} \omega_{-}\left(1+\bar{\lambda}_{0}\right)}{k_{z-} \omega_{0}}\right] . } \\
& \left(\frac{k_{z}}{\omega_{0}}-\frac{k_{z-}}{\omega_{-}}\right),
\end{aligned}
$$


and

$$
\Lambda_{A}=\Lambda /\left[\omega_{-} \bar{\lambda}_{-}\left(1+\bar{\lambda}_{-}\right)\right] \simeq-\Lambda /\left[\omega_{0} \bar{\lambda}_{-}\left(1+\bar{\lambda}_{-}\right)\right] .
$$

Combining the two coupled equations, Eqs. (6.38) and (6.45) we then derive the following dispersion relation for the parametric decay instabilities

$$
\left(\varepsilon_{A}-\tilde{\varepsilon}_{A}^{(3)}\right) \varepsilon_{S}=\Lambda_{A} \Lambda_{S} F^{2}(\bar{\lambda})\left|\bar{\phi}_{0}\right|^{2},
$$

where

$$
\tilde{\varepsilon}_{A}^{(3)}=\varepsilon_{A}^{(3)}+\Lambda_{A} \Lambda_{S} F(\bar{\lambda})\left|\bar{\phi}_{0}\right|^{2} .
$$

With $\mathrm{T}_{e} \gg \mathrm{T}_{i}$, the acoustic wave is weakly damped and we have the resonant decay instability. In this case, $\varepsilon_{A}^{(3)}$ can be neglected. Let $\omega_{S}=\omega_{s r}+i \gamma$ and $\omega_{-}=-\omega_{A}+i \gamma$, where $\omega_{\text {sr }}$ and $\omega_{A}=\omega_{0}-\omega_{\text {sr }}$ satsify, respectively, the dispersion relations for the ion acoustic and kinetic Alfvén waves. Equation (6.49) then reduces to

$$
\begin{gathered}
\frac{\partial \varepsilon_{s r}}{\partial \omega_{s r}} \frac{\partial \varepsilon_{A r}}{\partial \omega_{A}}\left(\gamma+\Gamma_{A}\right)\left(\gamma+\Gamma_{s}\right)=\left|\frac{\Lambda}{\omega_{0}}\right|^{2} \frac{F^{2}(\bar{\lambda})\left|\phi_{0}\right|^{2}}{\bar{\lambda}_{-}\left(1+\bar{\lambda}_{-}\right)}, \\
\frac{\partial \varepsilon_{s r}}{\partial \omega_{s r}}=\frac{2\left(1+\bar{\lambda}_{s}\right)}{\omega_{s r}},
\end{gathered}
$$


- 152 -

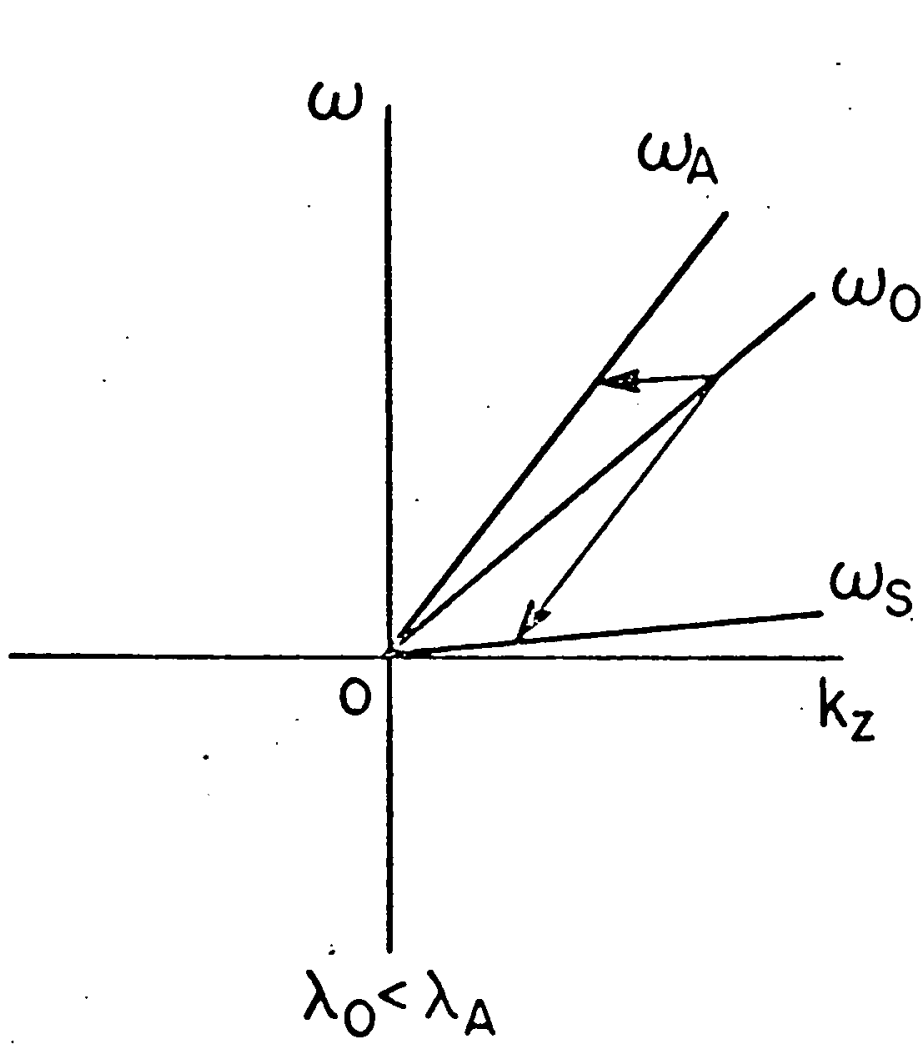

(a)

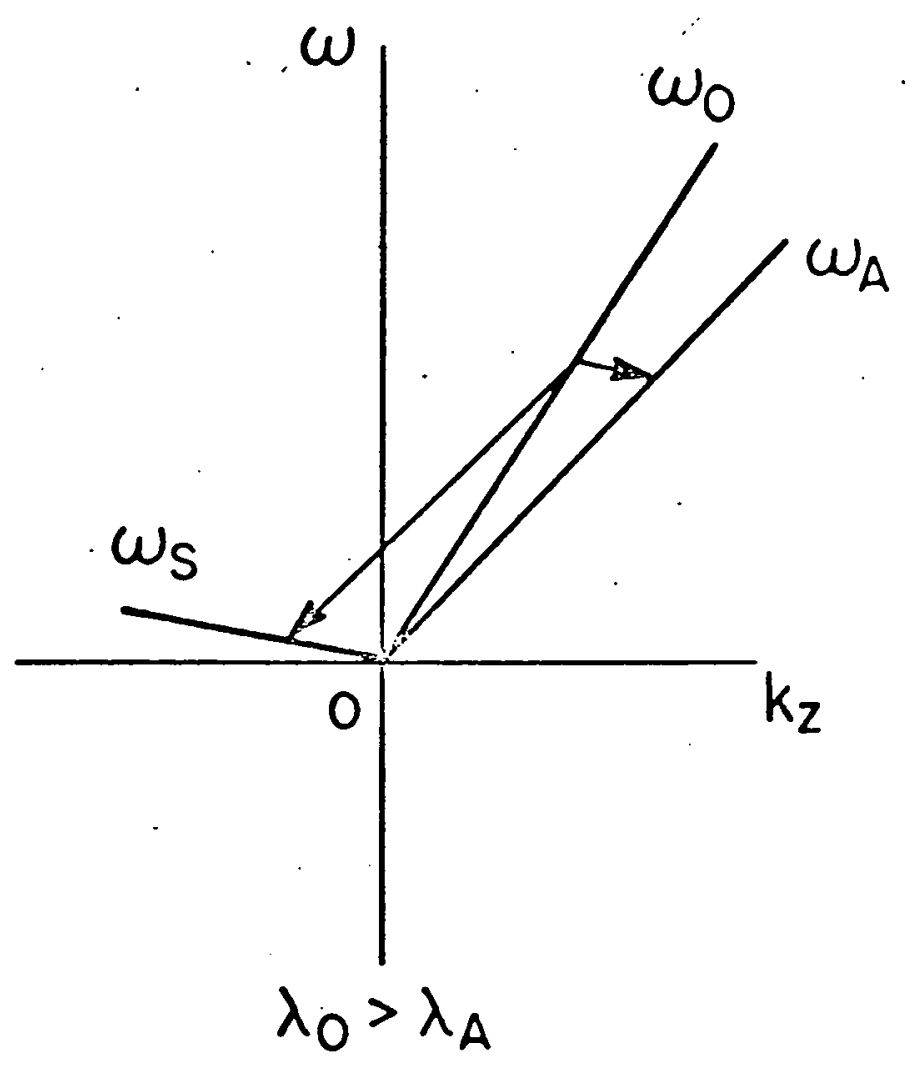

(b)

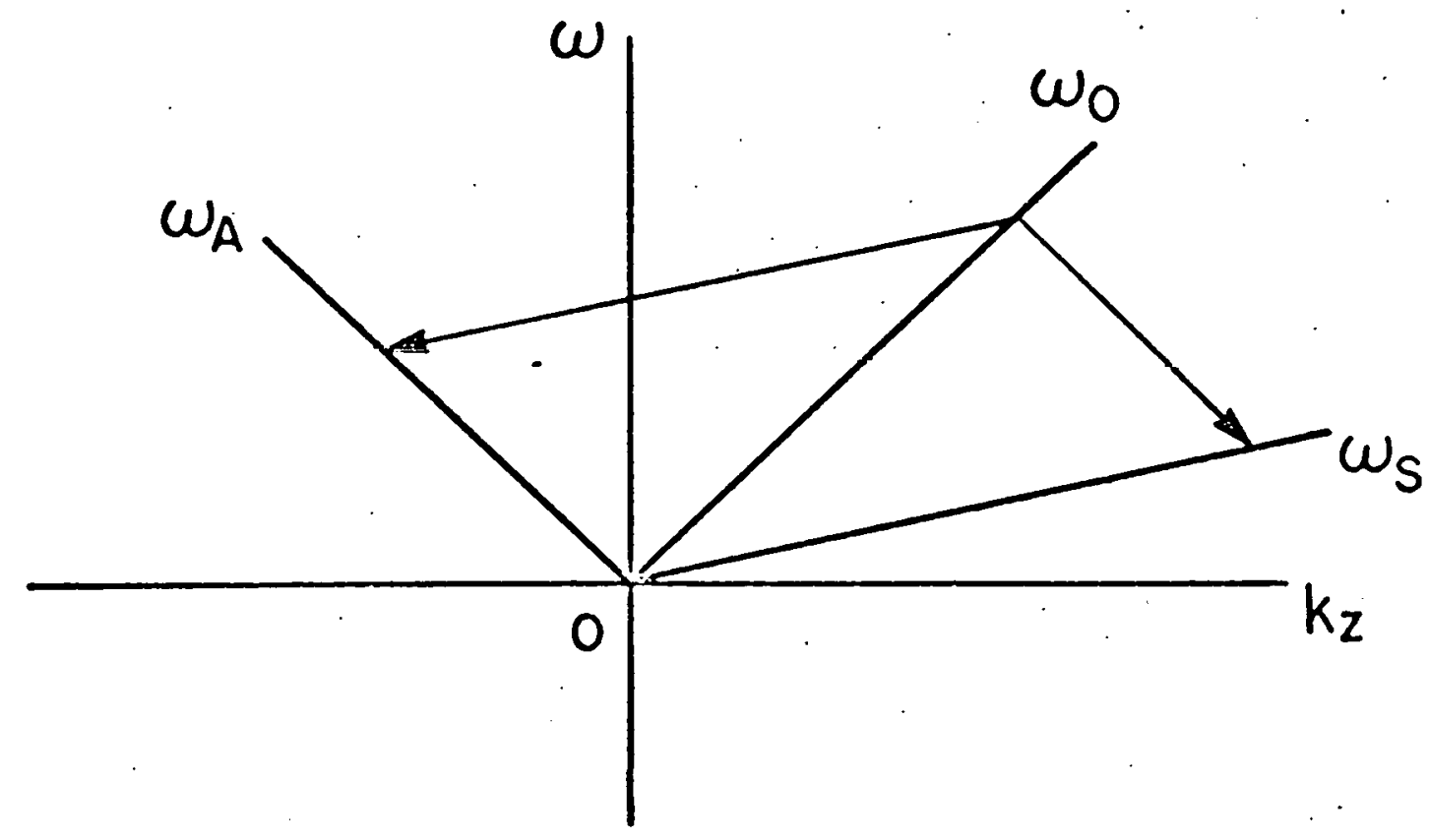

$$
\lambda_{0} \gtrless \lambda_{A}
$$

(c)

$$
\text { Fig.6.1 }
$$




$$
\frac{\partial \varepsilon_{\mathrm{Ar}}}{\partial \omega_{\mathrm{A}}}=\frac{2}{\omega_{\mathrm{A}}}
$$

and $\Gamma_{A^{\prime}}, \Gamma_{S}$ are the corresponding linear damping rates.

From Eq. (6.51), we can deduce the threshold pump field by letting $\gamma=0$. Well above the threshold field, the growth rate is given by

$\gamma_{D} \simeq \frac{\omega_{C i}}{4}\left(\frac{\omega_{s r}}{\omega_{A}}\right)^{1 / 2} \mid \frac{B_{10}}{B_{0}} \beta^{-1 / 2} \frac{|F(\bar{\lambda}) \sin \theta|}{\left[\left(1+\bar{\lambda}_{0}\right)\left(1+\bar{\lambda}_{-}\right)\left(1+\bar{\lambda}_{S}\right)\right]^{1 / 2}}$.

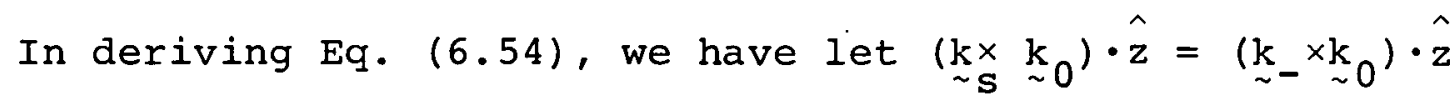

$=\mathrm{k}_{1-} \mathrm{k}_{10}$ sin $\theta$ and used the relation between $\mathrm{B}_{10}$ and $\bar{\phi}_{0}\left(=\right.$ e $\left.\phi_{0} / \mathrm{T}\right)$ expressed in $\mathrm{Eq} \cdot(6.5)$. The growth rate obtained here is larger than that of the ideal magnetohydrodynamic results by a factor of $\bar{\lambda} \omega_{C i} / \omega_{A}$.

This enhancement is expected because, due to the finite $\bar{\lambda}$ 's, nonlinear effects induced by the $\underset{\sim}{E} \times \mathbb{v}_{0}$ drifts of electrons and ions do not cancel each other to the order of $\bar{\lambda}$ in the case considered here; while only ion polarization arift (which is smaller than the $\underset{\sim}{E \times \sim_{0}}$ drift by a factor $\frac{\omega_{A}}{\omega_{C i}}$ ) contributes in the ideal magnetohydrodynamic limit. Furthermore, unlike the magnetohydrodynamic case in which only the backscattering is allowed, three different types of decay are possible here as illustrated in the $\omega-k_{z}$ diagram in Fig. 6.1. 
Note also since $F(\bar{\lambda}) \rightarrow 0$ as $\bar{\lambda}_{0} \rightarrow 0$, this decay process is pertinent to the pump wave being a kinetic Alfven wave.

Let us now consider the case with $\mathrm{T}_{\mathrm{e}} \lesssim 5 \mathrm{~T}_{i}$ such that the ion acoustic wave is heavily Landau damped by ions. In this case, the decay instability is made through nonlinear ion Landau damping, i.e., it is an ion-induced scattering process or, sometimes also called, a quasimode decay instability. From Eq. (6.49), the growth rate $\gamma_{\mathrm{N}}$ is obtained to be

$$
\gamma_{N}=-\frac{\omega_{A}}{2} \operatorname{Im}\left[\frac{\Lambda_{A} \Lambda_{S} F^{2}(\bar{\lambda})\left|\bar{\phi}_{0}\right|^{2}}{\varepsilon_{S}}+\ddot{\varepsilon}_{A}^{(3)}\right]-\Gamma_{A}
$$

Note, from Eq. $(6.50), \tilde{\varepsilon}_{\mathrm{A}}^{(3)}$ does not contribute to the growth rate and $\gamma_{N}$ further reduces to

$$
\gamma_{N}=\frac{\omega_{C i}}{8} B^{-1}\left|\frac{B_{10}}{B_{0}}\right|^{2}\left(\frac{\omega_{C i}}{\omega_{A}}\right) \frac{F^{2}\left(\bar{\lambda}_{)} \sin ^{2} \theta\right.}{\left(1+\bar{\lambda}_{0}\right)\left(1+\bar{\lambda}_{-}\right)} \frac{\operatorname{Im} \chi_{i}}{\left|\varepsilon_{S}\right|^{2}}-\Gamma_{A},
$$

which has its llaximum value at $\left|\omega_{s}\right| \sim\left|k_{z s} v_{T i}\right|$ and

$$
\left(\gamma_{N}\right)_{\max } \simeq \frac{\omega_{C i}}{8} \beta^{-1}\left|\frac{B_{10}}{B_{0}}\right|\left(\frac{\omega_{C i}}{\omega_{A}}\right) \frac{F^{2}\left(\bar{\lambda}_{)} \sin ^{2} \theta\right.}{\left(1+\bar{\lambda}_{0}\right)\left(1+\bar{\lambda}_{-}\right)} \frac{T_{e} / T_{i}}{\left(1+\bar{\lambda}_{S}\right)^{2}+\left(T_{e} / T_{i}\right)^{2}}-\Gamma_{A} .
$$

Main conclusions are: (i) the growth rate obtained here is larger than the classic magnetohydrodynamic value by a factor $\left(\bar{\lambda}_{C i} / \omega_{A}\right)^{2}$, (ii) the results are pertinent to the 
pump wave being a kinetic Alfvén wave and (iii), similar to the resonant decay instability, three types of decay are possible here (c.f. Fig.6.1). Note that the threshold pump field depends on $\Gamma_{A}$ which, in the collisionless regime, is mainly due to electron Landau damping and, typically, $\Gamma_{A} / \omega_{C i} \sim 0\left(10^{-2}\right)$. Then for a reasonable choice of other parameters; such as $\beta \sim 10^{-2}, \omega_{C i} / \omega_{A} \sim 10$ and $T_{e} \sim T_{i}$, the threshold amplitude of $B_{10},\left(B_{10}\right) \mathrm{th}^{/ B_{0}} \sim 10^{-2}$. 6.4 Solitary Wave of the Kinetic Alfvern Wave

Solitary wave is an nonlinear wave which propagates in a stationary form. Usually, such a stationary wave form is generated by a balance between a nonlinear steepening effect and a dispersion effect.

Since the MHD Alfvern wave does not have dispersion, to construct a solitary wave one should introduce either the finite frequency effect (Kakutami, 1974) or the finite wavenumber effect. We consider the latter example and construct the solitary wave of the kinetic Alfvên wave. (Hasegawa and Mima,1976)

The kinetic Alfvén wave has a dispersion for an oblique propagation. The nonlinearity originates from its compressible nature.

We make two assumptions. Firstly, that the plasma $B\left(=2 \mu_{0} \mathrm{nT} / \mathrm{B}_{0}^{2}\right)$ is small but much larger than the electron to ion mass ratio. The effect of a high $B$ correction is. studied recently by Yu and shukla (1978). Secondly, the variation exists in the $x-z$ planc, wherc $z$ is the direction of an ambient magnetic field $\underset{\sim}{\mathrm{B}}$ and $\mathrm{x}$ is a perpendicular direction. 
The low $\beta$ assumption allows one to use the two potential fields $\phi$ and $\psi$ to describe the electric field, $E_{x}=-\partial \phi / \partial x$ and $E_{z}=-\partial \psi / \partial z$. They produce only shear perturbations in the magnetic field; $B_{z}=B_{0}$ (const), $\mathrm{B}_{\mathrm{x}}=0$ and

$$
\frac{\partial B}{\partial t}=\frac{\partial^{2}}{\partial x \partial z}(\phi-\psi)
$$

The relevant field equations are the quasi-neutrality relation, for ion and electron densities $\mathrm{n}_{i}$ and $\mathrm{n}_{e}$

$$
n_{i}=n_{e}
$$

and Ampére's law for $J_{z}$, the current density in the $z$ direction

$$
\frac{\partial^{4}}{\partial x^{2} \partial z^{2}}(\phi-\psi)=\mu_{0} \frac{\partial^{2} J z}{\partial t \partial z}
$$

In a low $\beta$ plasma, the ion density is given by

$$
\frac{\partial n_{i}}{\partial t}-\frac{\partial}{\partial x}\left(\frac{m_{i}}{e B_{0}^{2}} \frac{\partial^{2} \phi}{\partial x \partial t} n_{i}\right)=0
$$

The electron density is obtained from the drift kinetic equation,

$$
v_{z} \frac{\partial f}{\partial z}+\frac{\partial}{\partial x}\left(v_{d x} f_{e}\right)+\frac{e}{m} \frac{\partial \phi}{\partial z} \frac{\partial f_{e}}{\partial v_{z}}=0
$$


Since the drift velocity $\underset{\sim d}{v_{d}}\left(=v_{z}{ }_{\sim}^{B} / B_{0}+E \times \hat{z} / B_{0}\right)$ has only the $y$-component, the second term does not contribute. Hence the electron density may be assumed to be given by the Boltzmann distribution,

$$
\mathrm{n}_{\mathrm{e}}=\mathrm{n}_{0} \exp \left(\mathrm{e} \psi / \mathrm{T}_{\mathrm{e}}\right)
$$

where $T_{e}$ is the electron temperature. In Eq. (6.60), the contribution of the ion parallel inertia term is ignored by the low $\beta$ assumption.

Because of the low $\beta$ assumption, the contribution of ions to the current density $J_{z}$ is negligible, hence $J_{z}$ is given by the electron density.

$$
\frac{\partial J_{z}}{\partial z}=\frac{\partial\left(e_{e}\right)}{\partial t}
$$

Equations $(6.59)$ to $(6.63)$ become dimensionless if we introduce new variables, $\xi=x / \rho_{s^{\prime}} \zeta=z \omega_{p i} / c, \tau=\omega_{c i} t$, $\bar{n}=n / n_{0}, \bar{\phi}=e \phi / T_{e^{\prime}}$ and $\bar{\psi}=e \psi / T_{e}$ where $\rho_{s}=\left(T_{e} / m_{i}\right)^{1 / 2} / \omega_{c i}$, $\omega_{\mathrm{ci}}=\mathrm{eB}_{0} / \mathrm{m}_{i}, \omega_{\mathrm{p} i}=\left(\mathrm{e}^{2} \mathrm{n}_{0} / \varepsilon_{0} \mathrm{~m}_{i}\right)^{1 / 2} ; \mathrm{n}_{0}$ is the unperturbed plasma density and $\mathrm{c}$ is the speed of light. We then consider a one-dimensional simple wave solution propagating obliquely with respect to the ambient magnetic field. The simple wave is chosen to be stationary with respect to a coordinate $\eta$, which is defined by

$$
n K_{x} \xi+K_{z} \zeta-\tau
$$


For the boundary conditions, we require that the number density perturbation $n(n)$ as well as its derivatives, $\partial \mathrm{n} / \partial \xi, \partial \mathrm{n} / \partial \zeta$ vanish as $\xi \rightarrow \pm \infty$, and $\zeta \rightarrow \pm \infty$. In terms of $\bar{\phi}$ and $\bar{\psi}$, this means $\bar{\psi}=\partial \bar{\psi} / \partial \xi=\partial \bar{\psi} / \partial \zeta=\partial^{2} \bar{\phi} / \partial \xi^{2}=$ $\partial^{3} \bar{\phi} / \partial \xi^{2} \partial \zeta=0$ as $\xi \rightarrow \pm \infty$ and $\zeta \rightarrow \pm \infty$.

Using these boundary conditions, and the variable $n$ Eqs. (6.59) and (6.60) can be integrated. If we then eliminate $\bar{\phi}, \bar{\psi}$ and $J_{z}$ from Eqs. $(6.59)$ to (6.63), we have the following nonlinear wave equation,

$$
\left(K_{z}^{2}-\bar{n}\right)(1-\bar{n})+K_{x}^{2} K_{z}^{2} \bar{n} \frac{d}{d n}\left(\frac{1}{\bar{n}} \frac{d \bar{n}}{d n}\right)=0
$$

The linear dispersion relation is obtained by substituting $\bar{n}=1+\delta n \exp (i k \eta)=\exp i k\left(k_{x} \xi+k_{z} \zeta-\tau\right) \equiv \exp i\left(k_{x} x+\right.$ $\left.\mathrm{k}_{z} z-\omega t\right):$

$$
i=k_{z}^{2}\left(1+k^{2} k_{x}^{2}\right)
$$

In terms of the original coordinates,

$$
\omega^{2}=k_{\mathrm{z}}^{2} \mathrm{v}_{\mathrm{A}}^{2}\left(I+\mathrm{k}_{\mathrm{x}}^{2} \rho_{\mathrm{s}}^{2}\right)
$$

This is the dispersion relation of the kinetic Alfven wave, Eq. (2.19). The related solitary wave solution is expected to have a structure scaled by the ion gyroradius $\rho_{i}$ in the perpendicular direction and to propagate almost in the parallel direction. 


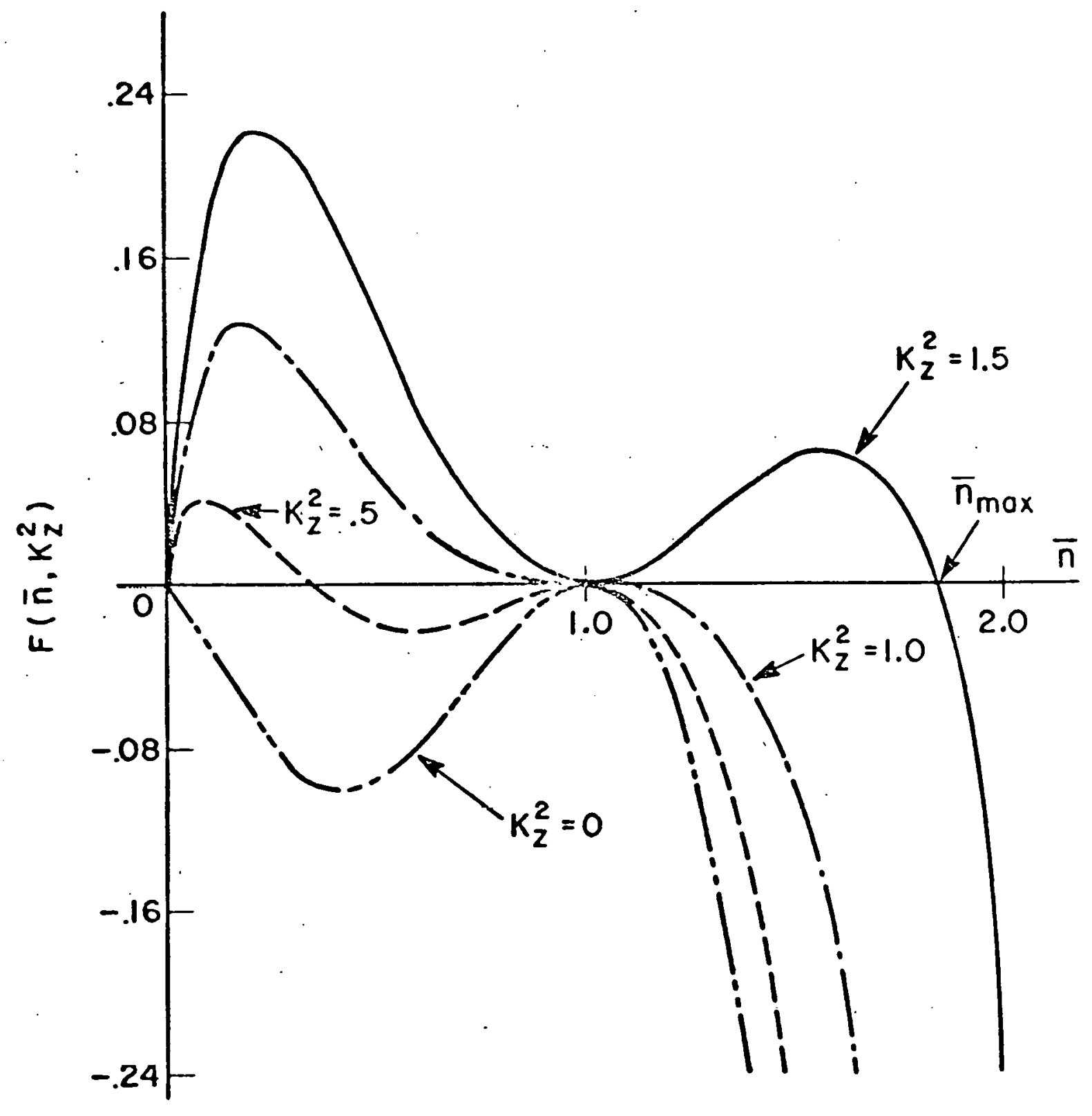

Fig. 6.2 
Equation $(6.65)$ can be readily integrated once by changing the independent variable from $n$ to $\bar{n}$. Using the condjtion $d \bar{n} / d n=0$ at $\bar{n}=1$, we obtain

$$
\begin{array}{r}
\left(\frac{\mathrm{d} \overline{\mathrm{n}}}{\mathrm{dn}}\right)^{2}=\frac{2 \overline{\mathrm{n}}}{\mathrm{K}_{\mathrm{x}}^{2} \mathrm{~K}_{\mathrm{z}}^{2}}\left[(1-\overline{\mathrm{n}})\left(\mathrm{K}_{\mathrm{z}}^{2}+\overline{\mathrm{n}}\right)+\left(1+\mathrm{K}_{\mathrm{z}}^{2}\right) \overline{\mathrm{n}} \ln \overline{\mathrm{n}}\right] \\
\equiv \frac{\mathrm{F}\left(\overline{\mathrm{n}}, \mathrm{K}_{\mathrm{z}}^{2}\right)}{\mathrm{K}_{\mathrm{x}}^{2} \mathrm{~K}_{\mathrm{z}}^{2}} .
\end{array}
$$

Expanding $F$ around $\bar{n}=1$ identifies $\bar{n}=1$ as a double root, a condition required for a solitary wave. The potential function $F\left(\bar{n}, \mathrm{~K}_{z}^{2}\right)$ is plotted in Fig. 6.2 for various values of $\mathrm{K}_{\mathrm{z}}^{2}$. The nonlinear wave(s) that we are seeking exist only for the range of density which makes $\mathrm{F} \geq 0$ including $\overline{\mathrm{n}}=1$. We see from this figure that there exists no range if $\mathrm{k}_{z}^{2}<1$, only one range, $0 \leq \overline{\mathrm{n}} \leq 1$, if $\mathrm{K}_{\mathrm{z}}^{2}=1$, and two ranges, $0 \leq \overline{\mathrm{n}} \leq 1$ and $1 \leq \bar{n} \leq \bar{n}_{\max }$ if $\mathrm{K}_{\mathrm{z}}^{2}>1 . \mathrm{k}_{\mathrm{z}}=1$ corresponds to a wave that propagates at the Alfvén speed in the $z$ direction, hence $\mathrm{K}_{\mathrm{z}}^{2}>\mathrm{I}\left(\mathrm{K}_{\mathrm{z}}^{2}<1\right)$ corresponds to a sub-Alfvénic (superAlfvénic) wave. For the sub-Alfvernic range there are two types of solitary wave solutions, one accompanying a density hump $(\bar{n} \geq 1)$ and the other density $\operatorname{dip}(\bar{n} \leq 1)$. Qualitatively this result agrees with the small amplitude result. However, the wave corresponding to $\bar{n} \leq 1$ has a 
minimum density of zero which is beyond the scope of our theoretical framework (since the Alfvén speed becomes infinity), and other effects, such as the finite frequency effect, need to be introduced to obtain a reasonable solution in this range of $\bar{n}$.

Consequently, we consider only the wave corresponding to $\overline{\mathrm{n}} \geq 1$. If $\mathrm{k}_{\mathrm{z}}^{2}$ deviates from unity by a small amount, say $M=\mathrm{K}_{\mathrm{z}}^{2}-1 \ll 1,(M>0)$, the maximum amplitude $\overline{\mathrm{n}}_{\max }$ of the solitary wave is correspondingly small. For such a case we can integrate Eq. (6.68) analytically for a small density perturbation, $\Delta=\bar{n}-1$, to give

$$
\Delta=\frac{3 M}{2} \operatorname{sech}^{2}\left(\frac{\sqrt{M} n}{2\left|K_{x} K_{z}\right|}\right)
$$

The corresponding magnetic field perturbation $B_{Y}$ (normalized by $B_{0}$ ) is obtained from Eq. (6.57),

$$
\frac{\mathrm{B}_{\mathrm{y}}}{\mathrm{B}_{0}} \equiv \overline{\mathrm{B}}_{\mathrm{y}}=-\frac{\mathrm{C}_{\mathrm{s}}}{\mathrm{v}_{\mathrm{A}}} \int(\overline{\mathrm{n}}-1) \frac{\mathrm{d} \eta}{\mathrm{K}_{\mathrm{X}} \mathrm{K}_{z}} \simeq-3 \sqrt{\mathrm{M}} \operatorname{sgn}\left(\mathrm{K}_{\mathrm{x}} \mathrm{K}_{\mathrm{z}}\right) \frac{\mathrm{C}_{\mathrm{s}}}{\mathrm{v}_{\mathrm{A}}} \tanh \left(\frac{\sqrt{\mathrm{M}} \eta}{2\left|\mathrm{~K}_{\mathrm{x}} \mathrm{K}_{z}\right|}\right)
$$

The wave accompanies a finite $\mathrm{x}$ component of the electric field as $\eta \rightarrow \pm \infty$, which is given by $\mathrm{E}_{\mathrm{X}}(n \rightarrow \pm \infty)=\mathrm{v}_{\mathrm{A}} \mathrm{K}_{\mathrm{Z}}^{-1} \mathrm{~B}_{\mathrm{Y}}(\eta \rightarrow \pm \infty)$. In general $\mathrm{E}_{\mathrm{x}}$ is given by 


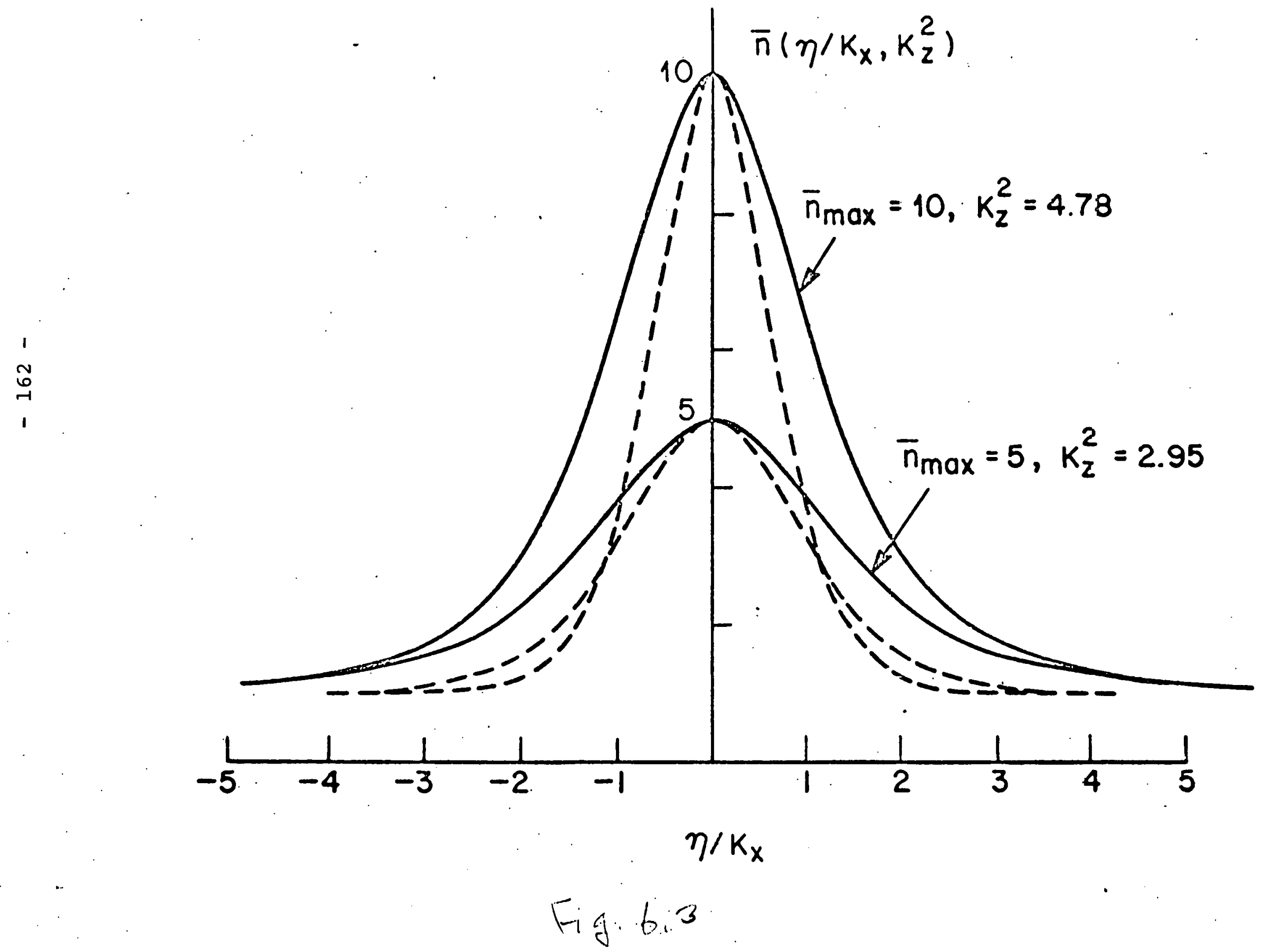


1
0
0
0
1
1

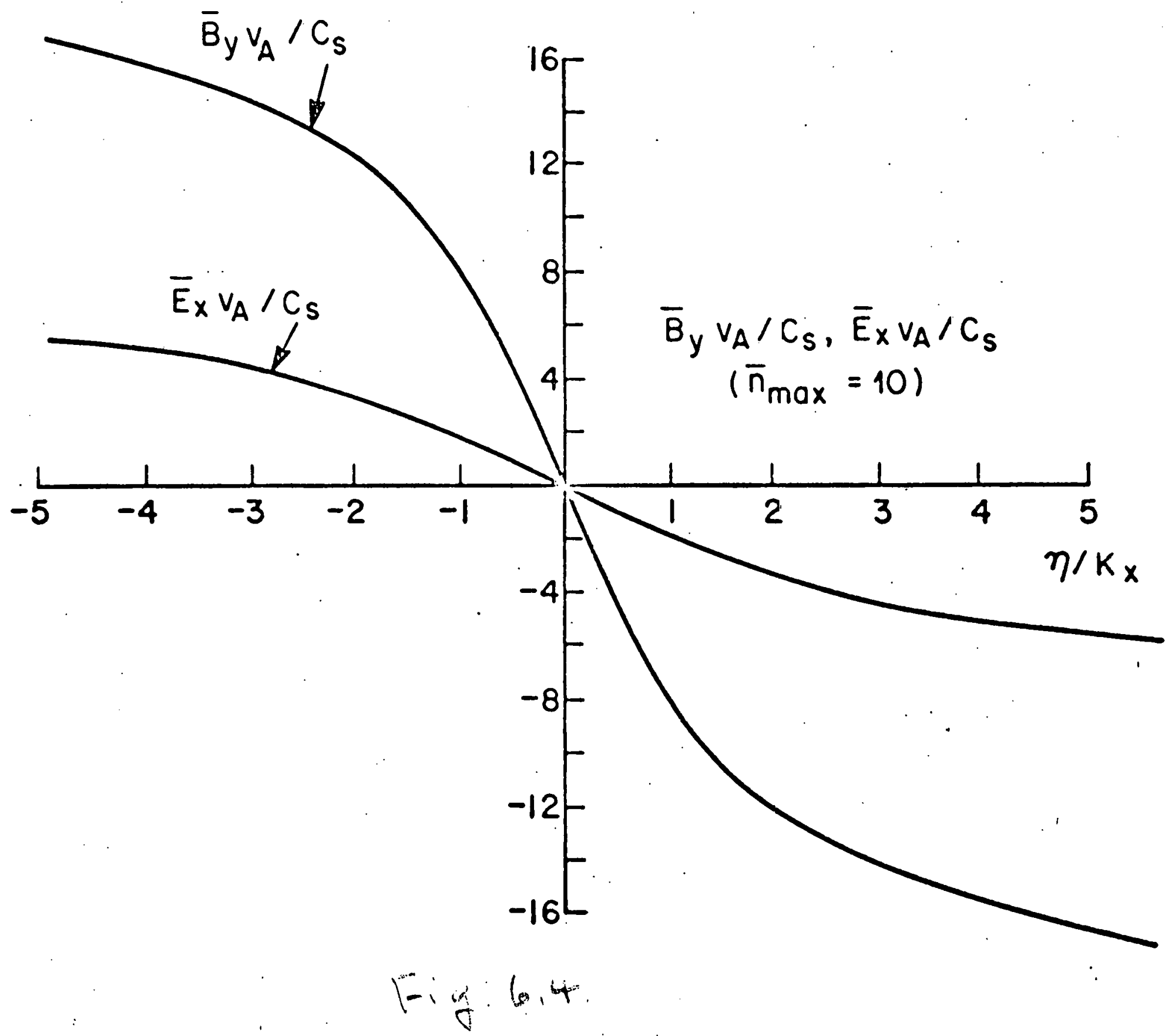




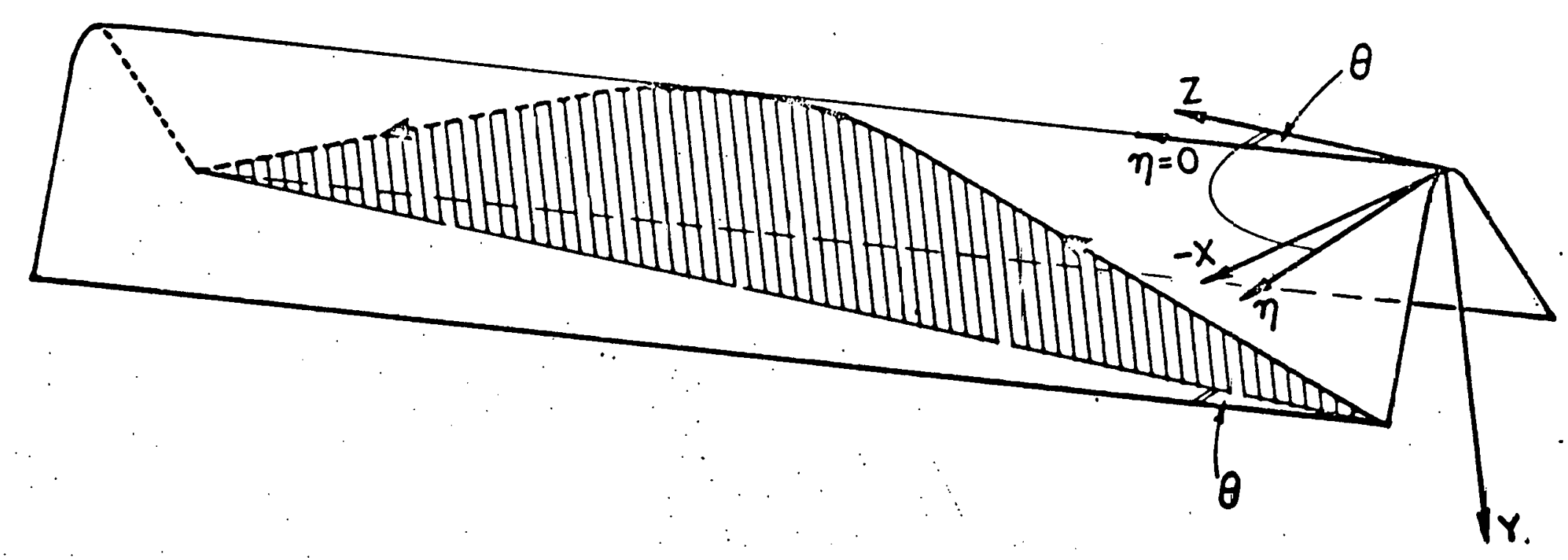

$$
\text { Fig.6.5 }
$$




$$
\begin{gathered}
-165- \\
\bar{E}_{X} \equiv \frac{E_{x}}{v_{A} B_{0}}=-\frac{c_{s}}{v_{A}} \int\left(\frac{\bar{n}-1}{\bar{n}}\right) \frac{d n}{K_{x}} .
\end{gathered}
$$

The present solution is exact to an arbitrary amplitude as long as the result is consistent with the low $\beta$ assumption. This means that the effective phase velocity of the wave should be larger than the ion sound speed $\mathrm{C}_{S}$. Hence the maximum allowable value of $K_{z}$ is $v_{A} / c_{s^{\prime}}$ or $K_{z}^{2} \lesssim \beta^{-1}$. For a large value of $\mathrm{k}_{\mathrm{z}}^{2}$, the peak density $\overline{\mathrm{n}}_{\max }$ is related to $\mathrm{K}_{\mathrm{z}}^{2}$ through $\mathrm{K}_{\mathrm{z}}^{2} \simeq \overline{\mathrm{n}}_{\max } / \ln \mathrm{n}_{\max }^{-}$. Hence the present result should be valid if $\bar{n}_{\max } \lesssim \beta^{-1}$.

Fig. 6.3 shows the density profiles for $\bar{n}_{\max }=10$ and $\mathrm{n}_{\max }=5$. which are obtained by numerically integrating Eq. (6.65). The dotted lines show the $\operatorname{sech}^{2}$ solutions that are fitted to the maximum density point. The exact solitary wave is seen to have a wider structure than the ideal $\operatorname{sech}^{2}$ solution. The corresponding values $\bar{B}_{y}$ and $\bar{E}_{y}$ are shown in Fig. 6.4. The integration constant is chosen so that these values vanish at the origin of the $n$ coordinate. As expected they have kink structures.

The structure of the total magnetic line of force is also interesting. Because $\mathrm{B}_{\mathrm{Y}}(\mathrm{n} \rightarrow \pm \infty) \rightarrow \pm$ constant, the line of force must be straight as $n \rightarrow \pm \infty$. Hence the projection of the field line into the $n-y$ plane is basically a triangular shape with a round top as shown in Fig. 6.5. 
The actual line of force exists in $y-z$ plane. Because the phase stationary line at $t=0$ exists on $\eta=0$, such $a$ line can be expressed by $\omega_{\mathrm{pi}} \mathrm{z} / \mathrm{c}=-\left(\mathrm{K}_{\mathrm{x}} / \mathrm{K}_{\mathrm{z}}\right) \mathrm{x} / \rho_{\mathrm{s}}$ namely $z=-\left(k_{x} / K_{z}\right)\left(v_{A} / c_{s}\right) x$. Hence the $z$ axis has an angle $\theta=-\tan ^{-1}\left(\mathrm{~K}_{\mathrm{z}} \mathrm{c}_{\mathrm{S}} / \mathrm{K}_{\mathrm{X}} \mathrm{v}_{\mathrm{A}}\right)$ with respect to $\mathrm{n}=0$ line. Therefore, the actual line of force follows the edge of an oblique slice of the triangular roof-like surface by the $y-z$ plane as shown by the arrowed solid curve in the figure. For a linear wave, $k_{x}$ approximately equals $\omega_{c i} / \omega>>$. I, as can be seen from Eq. (6.67). Hence, the angle $\theta$ is usually very small. 


\subsection{Modulational Instability and Envelope Soliton}

When a large amplitude wave propagates in a nonlinear dispersive medium, the amplitude modulation is known to grow under a certain condition. Such phenomena are called modulational instability. When the nonlinear dispersion relation for $\omega$ is given as $\omega(k, a)$, where $k$ is the wave number and $a$ is the amplitude of the wave, the nonlinear equation which describes the dynamics of a wave amplitude $\phi(x, t)$ with a monochromatic carrier frequency $\omega_{0}$ and wave number $k_{0}$ is given by (Karpman and Krushkal, 1969)

$$
i\left(\frac{\partial \phi}{\partial t}+v_{g} \frac{\partial \phi}{\partial x}\right)+\frac{1}{2} \frac{\partial v_{g}}{\partial k_{0}} \frac{\partial^{2} \phi}{\partial x^{2}}-\frac{\partial \omega_{0}}{\partial a}\left(|\phi|^{2}-\left|\phi_{0}\right|^{2}\right) \phi=0,
$$

where

$$
\begin{gathered}
v_{g}=\partial \omega /\left.\partial k\right|_{k=k_{0}, a=0} \\
\frac{\partial v_{g}}{\partial k}=\left.\frac{\partial^{2}(1)}{\partial k^{2}}\right|_{k=k_{0^{\prime}} \quad a=0} \\
\frac{\partial \omega_{0}}{\partial a}=\left.\frac{\partial \omega}{\partial a}\right|_{k=k_{0^{\prime}} \quad a=0}
\end{gathered}
$$

and $\left|\phi_{0}\right|$ is the magnitude of the stationary amplitude. The envelope function $\phi(x, t)$ is shown to be unstable if the product $\partial v_{g} / \partial k \cdot \partial \omega_{0} / \partial a$ has a negative sign. To prove this we put 


$$
\phi \equiv \sqrt{\zeta(x, t)} e^{i \sigma(x, t)},
$$

and obtain the dispersion relation $\Omega=\Omega(K)$ for small perturbations on $\rho$ and $\sigma$ such that

$$
\left(\begin{array}{l}
\rho \\
\sigma
\end{array}\right)=\left(\begin{array}{l}
\rho_{0} \\
0
\end{array}\right)+\operatorname{Re}\left(\begin{array}{l}
\rho_{1} \\
\sigma_{1}
\end{array}\right) \text { exp } i\left[k\left(x-v_{g} t\right)-\Omega t\right] .
$$

By substituting Eqs. (6.73) and (6.74) into (6.72), we derive the dispersion relation,

$$
\Omega^{2}=k^{4} / 4-\alpha \rho_{0} k^{2},
$$

where

$$
\alpha=-\frac{\partial \omega_{0}}{\partial a}, \frac{\partial v_{g}}{\partial k} .
$$

From Eq. (6.75), we see easily that an instability arises when $\alpha>0$. This means a small perturbation in the wave amplitude (envelope) grows in time. Such an instability is called modulational instability and is originally discovered in nonlinear optics.

The physical picture of the instability can be easily understood if we realize that Eq. (6.72) represents the Schrödinger equation of a quasi-particle in a potential field 
given by $\partial \omega_{0} / \partial a\left(|\phi|^{2}-\left|\phi_{0}\right|^{2}\right)$, if $\alpha>0$, the potential is attractive and the quasi-particles are trapped in the potential whose size is proportional to the quasi-particle density $|\phi|^{2}$ itself. Hence the more the quasi-particles are trapped, the deeper the trapping potential becomes, thus the perturbation grows.

This self-trapping process leads to a solitary wave solution for $|\phi|^{2}$. If we put $\left|\phi_{0}\right|^{2}=0$ and obtain an exact stationary solution of Eq. $(6.72)$ by imposing the condition that $|\phi|^{2} \rightarrow 0$ at $x \rightarrow \pm \infty$, we have, for $\alpha>0$,

$$
|\phi|^{2}=\rho_{0} \operatorname{sech}^{2}\left[\sqrt{\alpha \rho_{0}}\left(x-v_{g} t\right)\right]
$$

and

$$
\sigma=\frac{\alpha \rho_{0}}{2} t
$$

If $\alpha<0$, the solitary wave solution appears only under the condition that $|\phi|^{2} \rightarrow \rho_{1}(\neq 0)$ at $x \rightarrow \pm \infty$. The solitary wave appears in the form of an absence of wave energy (Hasegawa and Tappert, 1973);

$$
|\phi|^{2}=\rho_{1}\left\{1-\rho_{0} \operatorname{sech}^{2}\left[\sqrt{|\alpha| \rho_{1} \rho_{0}}\left(x-v_{g} t\right)\right]\right\},
$$

and

$$
\begin{gathered}
\sigma=\sin ^{-1} \frac{\sqrt{\rho_{0}} \tanh \left[\sqrt{|\alpha| \rho_{1} \rho_{0}}\left(x-v_{g} t\right)\right]}{\left\{1-\rho_{0} \operatorname{sech}^{2}\left[\sqrt{|\alpha| \rho_{0} \rho_{1}}\left(x-v_{g} t\right)\right]\right\}^{1 / 2}} \\
-\frac{|\alpha|}{2} \rho_{1}\left(3-\rho_{0}\right) t .
\end{gathered}
$$


Zakhalov and Shabat (1972) have shown that the nonlinear Schrödinger equation (6.72) can be solved exactly by using the inverse scattering method and shown that these solitary wave solutions are in fact solitons. That is the time asymptotic solution of Eq. (6.72) for an arbitrary initial condition can be expressed in terms of solitary wave solutions of Eq. $(6.81)$.

In contrast to the solitary wave solutions of the wave itself, these solitons are often called envelope solitons.

Now let us see whether the Alfven wave is modulationally stable or not. As we have seen, the modulational instability can be found by deriving the nonlinear dispersion relation $\omega(k, a)$ and finding the sign of $\partial v_{g} / \partial k \cdot \partial \omega / \partial a$. First we note that the Alfvén wave propagating in the direction of the ambient magnetic field does not have any dispersion, so that $\partial v_{g} / \partial k=0$. Hence to find the modulational instability we must consider the finite frequency correction. The dispersion relation as derived in Chapter II (Eq. (2.24)) then becomes

$$
\frac{c^{2} k^{2}}{\omega^{2}}=\frac{\omega_{p i}^{2}}{\omega_{c i}{ }^{\left(\omega_{c i}\right.}{ }^{-\omega)}} \text {. }
$$

Hence we see that $\partial v_{g} / \partial k=\partial^{2} \omega / \partial k^{2}<0$. Now to find $\partial \omega / \partial a$, we must apply a nonlinear perturbation theory. The perturbations should include amplitude to the third order. The most elegant method 
for this is the reductive perturbation theory developed by Taniuti and Yajima (1969). The reductive perturbation leads directly to the nonlinear schrödinger equation of the form $(6.72)$.

Leaving the detail of the perturbation processes to a reference (Hasegawa, 1972), we show here the nonlinear Schrödinger equation which describes the modulation amplitude of the Alfvén wave,

$$
\begin{aligned}
i\left(\frac{\partial \phi}{\partial t}+v_{g} \frac{\partial \phi}{\partial x}\right)+ & \frac{1}{2} \frac{\partial v_{g}}{\partial k} \frac{\partial^{2} \phi}{\partial x^{2}}-\frac{1}{4} \frac{k v_{A}^{2}}{v_{g}} \\
& \left(|\phi|^{2}-\left|\phi_{0}\right|^{2}\right) \phi=0 .
\end{aligned}
$$

We see here that $\partial \omega / \partial a_{a=0}=k v_{A}^{2} /\left(4 v_{g}\right)>0$. since $\partial v_{g} / \partial k<0$, we find that the Alfvern wave is modulationally unstable.

The nonlinear frequency shift $\partial \omega / \partial a$ is closely related to the ponderomotive force. The modification of the background plasma density due to the electromagnetic wave pressure (ponderomotive force) leads to the local shift of the wave frequency. In the case of the Alfvern wave, the wave energy is primarily magnetic and the corresponding ponderomotive force becomes negative; this means that the plasma is attracted to the region of larger intensity of wave magnetic field (Kaufman et al., 1978). This leads to the modulational instability in the region of negative $\partial \mathrm{v}_{g} / \partial \mathrm{k}$ which is opposite to the case of the well known Langmuir wave where the ponderomotive force is positive. 


\section{REFERENCES FOR CHAPTER VI}

Boley, F.I. and Forman, P.R., Steepening of large amplitude Alfvén waves, Phys. Rev. Lett. 12, 384 (1964).

Ferraro, V.C.A., Hydromagnetic waves in a rare ionized gas and galastic magnetic fields, Proc. Roy. Soc. A233, 310 (1956).

Hasegawa, A., Theory and Computer Experiment on Self-trapping Instability of Plasma Cyclobon Waves, Phys. Fluids 15 , 870 (1972).

Hasegawa, A. and Chen L., Kinetic Processes in Plasma Heating by Resonant Mode Conversion of Alfvén Wave, Phys. Fluids 19, 1924 (1976).

Hasegawa, A. and Mima, K., Anomalous Transparent Produced by Kinetic Alfvén Wave Turbulence, J.. Geophys. Res. 83, 1117 (1978).

Hasegawa, A. and Mima, K., Exact Solitary Alfvén Wave, Phys. Rev. Lett. 37, 690 (1976).

Hasegawa, A., and Tappert, F., Transmission of Stationary Optical Pulses in Dispersive Dielectric Fibers, II Normal Dispersion, Appl. Phys. Lett. 23, 171 (1973). 
Kakutani, T., Plasma Waves in Long Wave Approximation, Prog. Theor. Phys., Suppl. No. 55 (1974).

Karpman, V.I. and Krushkal, E.M., Modulated Wave in Nonlinear Dispersive Media, Sov. Phys. JETP 28, 277 (1969).

Kaufman, A. N., Cary, J. R. and Pereira, N. R., Universal

Formula for the Quasi-Static Second Order Density

Perturbation by a Cold Mangetoplasma Wave, Proc. 3rd

Topical Conf. on R.F. Plasma Heating, Passadena (1978) .

Montgomery, D., Development of hydromagnetic shocks from large amplitude Alfvén waves, Phys. Rev. Lett. 2 , 36 (1959)..

Sagdeev, R.Z. and Galeev, A.A., Nonlinear Plasma Theory, ed. by T. M. O'Neil and D. L. Book, New York, W. A. Benjamin, Inc., 1969.

Shaffman, P.G., Propagation of a solitary wave along a magnetic field in a cold collision-free plasma, J. Fluid Mech. Il, 16 (1961).

Taniuti, T. and Yajima, N., Perturbation Method for Nonlinear Wave Modulation, I, J. Math. Phys. 10, 1369 (1969).

Yu, M.Y. and Shukla, P.K., Finite Amplitude Solitary Alfvén Waves, Phys. Fluids 21, 1457 (1978).

Zakhalov, V.E. and Shabat A.B., Exact Theory of Two-Dimensional Self-Focusing and One-Dimensional Self-Modulation of Waves in Nonlinear Media, Sov. Phys. JETP 34, 62 (1972). 


\section{OTHER REFERENCES FOR CHAPTER VI}

Aleksin V.F. and Khodusov, V.D., On Magnetohydrodynamic Wave Kinetics. III. Ukr. Fiz, Zh. 18, 1707-16 (1973).

Barnes, A., On the Nonexistence of Plane-polarized Large Amplitude Alfvén Waves. J. Geophys. Res. 81, 281-2 (1976).

Barnes A. and Suffolk, G.C.J., Relativistic Kinetic Theory of the Large-amplitude Transverse Alfvén Wave. J. Plasma Phys. (GB) 모 315-29 (1971).

Bate, R.T. and Wisseman, W.R., Second-harmonic Generation by Damped Alfvén Waves and Helicons in Pure and Doped Bismuth. Phys. Rev. 181, 763-73 (1969).

Belcher, J.W. and Davis, L. Jr., Large-amplitude Alfvén Waves in the Interplanetary Medium. II. J. Geophys. Res. 76 , 3534-63 (1971).

Berezin, Yu.A., Nonlinear Motions in an Anisotropic Plasma. Zh. Eksp. and Teor. Fiz. 61, 1877-81 (1971). Cohen, R.H., Mode Decay and Evolution of the Solar Wind Alfvén Wave Spectrum. J. Geophys. Res. 80, 3678-80 (1975). Cohen, R.H. and Dewar, R.L., On the Backscatter Instability. of Solar Wind Alfvén Waves. J. Geophys. Res. 79, 4174-8 (1974).

Cramer, N.F. and Hewitt, R.G.L., Parametric Excitation of Compressional Alfvén Waves. Phys. Lett. A $\underline{54 A}, 493-4$ (1975). 


$$
\begin{array}{r}
-175- \\
V I-0-2
\end{array}
$$

Dokuchaev, V.P., The Theory of Magnetic Bremsstrahlung From Alfvén Waves in an Incompressible Medium. Magn. Gidrodinamika 2 , 23-30 (1970).

Elachi, C., Parametric Interactions Between Alfvén Waves and Sonic Waves. IEEE Trans. Antennas and Propag. AP-21, 907-9 (1973).

Elfimov, A.G. and Nekrasov, F.M., Nonlinear Saturation in Parametric Excitation of Alfvén Waves. Sov. Phys.-Tech. Phys. 20, 1300-2 (1975).

Elfimov, A.G. and Nekrasov, F.M., Excitation of Alfvén Waves in Decay Instabilities. Sov. Phys.-Tech. Phys. 19, 9-11 (1974).

Elfimov, A.G. and Nekrasov, F.M., Contribution to the Theory of Hydrodynamic Plasma Stability in the Field of a Finite-Amplitude Alfvén Wave. Nucl. Fusion 12, 201-6 (1972)

Fu, K.Y., Nonlinear Mode-Mode Coupling of Alfvén Waves in the Interstellar Medium. Plasma Phys. (GB) 15, 57-76 $(1973)$.

Grebinskii, A.S., The Quasilinear Theory of the Growth of Alfvén Waves in a Nonuniform Medium. Soviet Phys.-Tech. Phys. $38,1190-3$ (1968).

Hollweg, J.V. and Skadron, G., Fokker-Planck Theory for Cosmic Ray Diffusion in the Presence of Alfvén Waves. I. Theory. J. Geophys. Res. 80, 2701-7 (1975). 


$$
\begin{array}{r}
-176- \\
V I-0-3
\end{array}
$$

Hollweg, J.V., Wave Coupling Between Solar Wind Protons and Helium. Am. Geophys. Union 54, 1190 (1973).

Hollweg, J.V., Nonlinear Landau Damping of Alfvén Waves. Phys. Rev. Lett. 27, 1349-52 (1971).

Hollweg, J.V., Density Fluctuations Driven by Alfvén Waves. J. Geophys. Res. 76, 5155-61 (1971).

Hung, N.T., Parametric Excitation of Alfven and Acoustic Waves. J. Plasma Phys. (GB) 12, 445-53 (1974).

Inutake, M. and Kuriki, K., Experiments on Alfvén Wave and Shock Wave in a Plasma Wind Tunnel. Bull. Inst. Space and Aeronaut. Sci. Univ. Tokyo B 10, 799-817 (1974). Ionson, J.A. and Ong, R.S.B., The Long Time Behavior of a Finite Amplitude Shear Alfvén Wave in a Warm Plasma. Plasma Phys. (GB) 18, 809-19 (1976).

Kakutani, T., Plasma Waves in the Long Wave Approximation. Prog. Theor. Phys. Suppl. 55, 97-119 (1974).

Kaw, P.K., Parametric Excitation of Electromagnetic Waves in Magnetized Plasmas. Advances in Plasma Physics $\underline{6}$, 207-36 (1976).

Kennel, C.F. and Pellat, R., Relativistic Nonlinear Plasma Waves in a Magnetic Field. J. Plasma Phys. (GB) 15, 335-55 (1976).

Lashmore-Davies, C.N., Modulated Instability of a Finite Amplitude Alfvén Wave. Phys. Fluids 19, 587-9 (1976). Lashmore-Davies, C.N. and Ong, R.S.B., Parametric Excitation of Alfvén and Ion Acoustic Waves. Phys. Rev. Lett. 32 , $1172-5$ (1974). 


$$
\begin{array}{r}
-177- \\
V I-0-4
\end{array}
$$

Lee, M.A. and Volk, H.J., Hydromagnetic Waves and CosmicRay Diffusion Theory. Astrophys. J. 198, 485-92 (1975). Lee, M.A. and Volk, H.J., Damping and Nonlinear Wave-Particle Interactions of Alfvên-Waves in the Solar Wind. Astrophys and Space Sci. 24, 31-49 (1973).

Lehane, J.A. and Paolini, F.J., Observation of Parameteric Amplification of Alfvén Waves. Phys. Lett. A 32A, 409-10 $(1970)$

Leonard, B.P., Magnetic Structure of Ionizing Shock Waves. III. Normal Shocks. J. Plasma Phys. (GB) 7, 177-85 (1972). Leonard, B.P., Magnetic Structure of Ionizing Shock Waves. II. Oblique Shocks. J. Plasma Phys. (GB) 7, 157-76 (1972).

Leonard, B.P., Magnetic Structure of Ionizing Shock Waves. I. Skew Shocks. J. Plasma Phys. (GB) I, 133-55 (1972). Mckenzie, J.F. and Bornatici, M., Effect of Sound Waves, Alfvén Waves, and Heat Flow on Interplanetary shock Waves. J. Geophys. Res. 79, 4589-94 (1974). Mikhailovskii, A.B.; Pietviashvili, V.I.; and Fridman, A.M., The Alfvén Soliton. JETP Letters, 24, 53-6 (1976). Mikhailovskii, A.B. and Pokhotelov, O.A., Influence of Whistlers and Ion-Cyclotron Oscillations on Excitation of Alfvén-Waves in Magnetospheric Plasma. Fiz. Plazmy I. 1004-11 (1975). 


$$
\begin{gathered}
-178- \\
V I-0-5
\end{gathered}
$$

Mio, K.; Ogino, T.; Minami, K.; and Takeda, S., Modified Nonlinear Schrödinger Equation for Alfvén Waves Propagating. Along the Magnetic Field in Cold Plasmas. J. Phys. Soc. Jap. 41, 265-71 (1976).

Nguyen, H., Nonlinear Decay of a Large-Amplitude IonAcoustic Wave. J. Plasma Phys. (GB) 14, 347-51 (1975). Ohsawa, Y. and Nozaki, K., Propagation of Solitary Pulses in Interactions of Plasma Waves. II. J. Phys. Soc. Jap. $36,591-5 \quad(1974)$.

Patterson, B., Exact Nonlinear Evolution of Al्fvén-Mode in the Guiding Center Model, Phys. Fluids 14, 1127 (1971).

Petrukhin, N.S., On the Mechanism of Sound Pulses Generation in the Solar Atmosphere. Pis'ma V Astron. $\mathrm{Zh}$. $1,32-6$ (1975) .

Petrukhin, N.S., Parametric Amplification of Alfvén Waves in the Solar Atmosphere. Astron. Zh. 5l, 571-6 (1974). Pozwolski, A., Parametric Amplification of Alfvén Waves. onde Filect.r. 53, 412-14 (1973). Rogister, A., Propagation of Alfvén waves in Ion-Sound Turbulent Plasma. J. Plasma Phys. (GB) 6, 309-23 (1971). Saka, 0. and Kitamura T., Turbulent Spectra of the Transverse Alfvén Waves in the Corotating Solar wind structure. Rep. Ionds. and Space Res. Jap. 29, 127-32 (1975) . Scholer, M. and Belcher, J.W., The Effect of Alfvén Waves on MHD Fast Shocks. Solar Phys. 16, 472-83 (1971). 


$$
\begin{array}{r}
-179- \\
V I-0-6
\end{array}
$$

Selim, A.A. and Krishan, S., Amplitude Dispersion in the Electromagnetic Modes. Phys. Fluids 14, 1166-70 (1971). Sharma, S.K. and Tripathi, V.K., Alfvén Wave Second-Harmonic Generation in Bismuth' Kinetic Theory. J. Appl. Phys. 453, 2155-60 (1972).

Tamoikin, V.V. and Fainshtein, S.M., Kinetic Equations for Sound and Alfvén Waves in a Plasma with Random Inhomogeneities. Sov. Phys. JETP, 71, 531-6 (1976). Tamoikin, V.V. and Fainshtein, S.M., Stimulated MandelstamBrillouin Scattering on Propagation of Alfvén Waves in a Plasma with Random Inhomogeneities. Sov. Phys. JETP, 68, 948-55 (1975). Tsytovich, V.N. and Kaplan, S.A., Relativistic Turbulent

Plasma in Pulsars. Astrophysics, $8,441-60$ (1972). Vahala, G. and Montgomery, D., Parametric Amplification of Alfvén Waves. Phys. Fluids 14, 1137-40 (1971). Valley, G.C., Scattering of Alfvén Waves by Random Density Fluctuations. Astrophys. J. 188, 181-9 (1974). Valley, G.C., Scattering of Alfvén Waves by Random Density Fluctuations (In the Solar Wind). American Geophys. Union, 54, 1192 (1973).

Williams, D.J., Magnetohydrodynamic Accretion and the Instability of Smooth Trans-Alfvenic Flow. Mon. Not. R. Astron. Soc. (GB) 171, 537-49 (1975). Yuang-Chung, Chin and Wentzel, D.G., Nonlinear Dissipation of Alfvén Waves. Astrophys. and Space Sci. 16, 465-77 (1972). 


\section{PLASMA HEATING BY ALFVÉN WAVE}

\subsection{Introduction}

In this chapter we consider plasma heating by the Alfvén wave. As we have seen in an homogeneous plasma, an externally applied electromagnetic field with frequency $\omega_{0}$ faces spatial resonance absorption at the Alfvén resonant surface $r=r_{0}$ given by

$$
\omega_{0}=k_{\|}\left(r_{0}\right) v_{A}\left(r_{0}\right)
$$

If a proper choice of paraneters is made, the absorption rate approaches $100 \%$, that is, the wave energy is deposited into the plasma at the same rate as the frequency of the wave. Plasma heating by utilizing this absorption has been proposed independently by Grossmann and Tataronis (1973) and Hasegawa and chen (1974). The absorbed energy is shown to be converted to the kinetic Alfvén wave. The plasma is heated when the kinetic Alfven wave is dissipated by the particles either by collision damping, Landau damping, and/or nonlinear effects (Hasegawa and Chen, 1975). We derive the rate of absorption in 7.2 , the heating rate of each species in 7.3 and some design example in 7.4.

\subsection{Resonant Absorption and Energy Deposition Rate}

As we discussed in Chapter IV, in an inhomogeneous plasma, the Alfvén wave has continuous spectrum. If a monocirronatic rave o: ho osciilating nagnetic ficla externally to an inhomogeneous plasma the wave energy is absorbed at the resonant surface. The absorption rate can be obtained by using the idcal MHD equations. This rate can be shown 
to be the same even if the kinetic theory is used if the kinetic Alfvén wave, which is excited by the mode conversion, propagates into the plasma and is eventually absorbed by wave particle interactions. Here, we calculate the energy absorption rate using a model boundary condition for a conceivable plasma. We take planar geometry as shown in Fig. 7.1 in which the inhomogeneous plasma is located in $x \geq 0$. The current in the antenna coil is represented by a surface current $J_{S}$ at $x=-h \cdot x=x_{0}$ represents the Alfvén resonant surface. The plasma is assumed to be uniform in $x \geq a$. The equilibrium properties of the plasma (mass density $\rho$, pressure $P$, and the confining magnetic field $\underset{\sim}{B}$ ) vary only in the $x$ direction. $\underset{\sim}{B}(\mathrm{x})$ has a shear component; i.e.,

$$
\underset{\sim}{B}(x)=B_{z}(x) e_{z}+B_{Y}(x) e_{y} \cdot
$$

(In a toroidal plasma, $x, y$, and $\mathbf{z}$ correspond to radial, poloidal and toroidal direction, respectively.) $\mathrm{P}(\mathrm{x})$ and $\underset{\sim}{B}(x)$ satisfy the equilibrium condition

$$
\mathrm{d} / \mathrm{dx}\left(\mathrm{p}+\mathrm{B}^{2} / 2 \mu_{0}\right)=0
$$

Iinearizing the ideal magnetohydrodynamic equations, including the compressibility effect, from the equation of motion we have

$$
\left.\mu_{0} \rho \underset{\sim}{\xi}-(\underset{\sim}{B} \cdot \nabla){ }^{2} \underset{\sim}{\xi}=-\mu_{0} \nabla \tilde{p}-\underset{\sim}{B} \underset{\sim}{B} \cdot \nabla\right)(\nabla \cdot \underset{\sim}{\xi}),
$$

where $\xi$ is the fluid displacement vector and 
$\underset{\sim}{B}$. the perturbed magnetic field, is related to $\underset{\sim}{\xi}$ through the Maxwell equations

$$
\underset{\sim}{\mathrm{B}}=(\underset{\sim}{\mathrm{B}} \cdot \nabla) \underset{\sim}{\xi}-{\underset{\sim}{\mathrm{B}}}_{0}(\underset{\sim}{\xi} \cdot \nabla) \underset{\sim}{\mathrm{B}}
$$

Combining the adiabatic equation of state and the continuity equation, we can express the perturbed plasma pressure $p$ in terms of $\underset{\sim}{\xi}$, as

$$
\mathrm{p}=-\xi_{\mathrm{x}} \mathrm{dP} / \mathrm{dx}-\gamma \mathrm{P}(\nabla \cdot \underset{\sim}{\xi}) .
$$

Assuming perturbations of the form

$$
\underline{\xi}=\underset{\sim}{\xi}(\mathrm{x}) \exp \left[i\left(k_{z} z+k_{y} y-\omega t\right)\right],
$$

with $\omega=\omega_{0}+i \delta\left(\delta=0^{+}\right)$and adopting the local rectangular coordinates with $\underset{\sim}{\mathrm{e}}{ }_{\|}={\underset{\sim}{\mathrm{B}}}_{0} /\left|{\underset{\sim}{\mathrm{B}}}_{0}\right|$ and $\underset{\sim}{\mathrm{e}}={\underset{\sim}{\mathrm{e}} \|}_{\|} \times \underset{\sim}{\mathrm{e}} \mathrm{x}^{\prime} \mathrm{Eq} \cdot(7.2)$ then becomes, for each component,

$$
\begin{aligned}
& \varepsilon \xi_{\|}=i k_{\|} \mu_{0} \tilde{p}+i B_{0}^{2} k_{\|}\left(i k_{\|} \xi_{\|}+i k_{\perp} \xi_{\perp}+d \xi_{x} / d x\right) \\
& \varepsilon \xi_{\perp}=i k_{\perp} \mu_{0} \tilde{p}
\end{aligned}
$$

and

$$
\varepsilon \xi_{x}=\mu_{0} d \tilde{p} / \mathrm{d} \mathbf{x}
$$

Here,

$$
\begin{aligned}
& \varepsilon(x)=\omega^{2} \mu_{0} \rho-k_{\|}^{2} B_{0}^{2}, \\
& \tilde{p}=p+\underset{\sim}{B} \cdot{\underset{\sim}{B} 0}_{0} / \mu_{0},
\end{aligned}
$$$$
k_{\|}(x) B_{0}(x)=k_{z} B_{0 z}+k_{y} B_{0 y} \text {, and } k_{1}(x) B_{0}(x)=k_{y} B_{0 z}-k_{z}{ }_{0 y}
$$

Eqs. (7.3) and (7.4), we can express $\xi_{\perp}(x)$ in terms of $\xi_{x}(x)$, as 


$$
\xi_{\perp}(x)=\frac{i \alpha k_{\perp} B_{0}^{2}}{\alpha k_{\perp}^{2} B_{0}^{2}-\varepsilon} \frac{d \xi_{x}}{d x} .
$$

Here,

$$
\alpha(x)=1+\frac{\omega^{2} C_{s}^{2}}{v_{A}^{2}\left(\omega^{2}-C_{s}^{2} v_{I I}^{2}\right)}
$$

Substituting Eqs. (7.6) and (7.8) into Eq. (7.7), we then arrive at the wave equation for $\xi_{x}$.

$$
\frac{d}{d x}\left(\frac{\varepsilon \alpha B_{0}^{2}}{\alpha k_{\perp}^{2} B_{0}^{2}-\varepsilon} \frac{d \xi_{x}}{d x}\right)-\varepsilon \xi_{x}=0 .
$$

Equation (7.10) contains the shear Alfvên, the magnetosonic, and the ion-acoustic waves. Due to the nonuniformities, these three waves are coupled. Note that Eq. (7.10) has a singular solution at the resonant point $\mathrm{x}=\mathrm{x}_{0}$ where $\varepsilon=0$. This singularity then causes the wave phase mixing and, hence, the energy of the excited wave is dissipated. Equation (7.10) is a generalized form of Eq. (3.5) in that the effect of compressibility is included. When $C_{s} \rightarrow \infty$ and $k_{\perp}=0$, this equation reduces to the ideal Alfvén wave equation (3.5). We also note here that near the spatial resonance where $\varepsilon \simeq 0, \mathrm{Eq} \cdot(7.10)$ is reduced to 


$$
\frac{d}{d x}\left(\varepsilon \frac{d \xi}{d x}\right)-\varepsilon k_{\perp}^{2} \xi_{x}=0
$$

hence it recovers the structure of Eq. (3.6). This indicates that near the Alfvern resonance, the effect of compressibility becomes irrelevant. In cire sujsecuenc analysig ic shall assume low $\beta$ plasma is $c_{s}^{2} \ll v_{\lambda}^{2}$ so that $\omega_{0}^{2} \gg c_{s}^{2} k_{\| 1}^{2}$ and $\alpha(x) \simeq 1+c_{s}^{2} / v_{A}^{2} \sim 0(1)$.

Let us now obtain the energy absorption rate $\mathrm{dw} / \mathrm{dt}$. From the power conservation law, dw/dt should be equal to the difference in power flow toward the plasma before and after it reaches the resonance surface $x_{0}$. The total power flow $P$ in a cross sectional area $S=I_{y} L_{z}$ is given by

$$
\begin{aligned}
P & =\frac{L_{y^{L} z}}{2} \operatorname{Re}\left(\underset{\sim}{E \times H_{\sim}^{*}}{ }_{\sim}^{*} P^{*}\right) \\
& =\frac{L_{y} L_{z}}{2} \operatorname{Re} i \omega_{0} \xi_{x}^{*} \tilde{p} \\
& =-\frac{L_{y} z_{z} \omega_{0}}{2} \operatorname{Im} \xi_{x}^{*} \frac{\varepsilon}{k_{\perp}^{2} \mu_{0}} \frac{\partial \zeta_{x}}{\partial x} .
\end{aligned}
$$

If we note that $\xi_{x}$ has a logarithmic solution near $x=x_{0}$ ' such that

$$
\xi_{x}=c \ln \left(x-x_{0}+i \delta\right)
$$

Eq. (7.12) indicates that the power flow is discontinuous (but not divergent) across the plane $x=x_{0}$. Hence the absorption rate is obtained as, 
$\operatorname{VACUUM}(x<0) \underbrace{\Delta \in(x)} \operatorname{PLASMA}(x>0)$

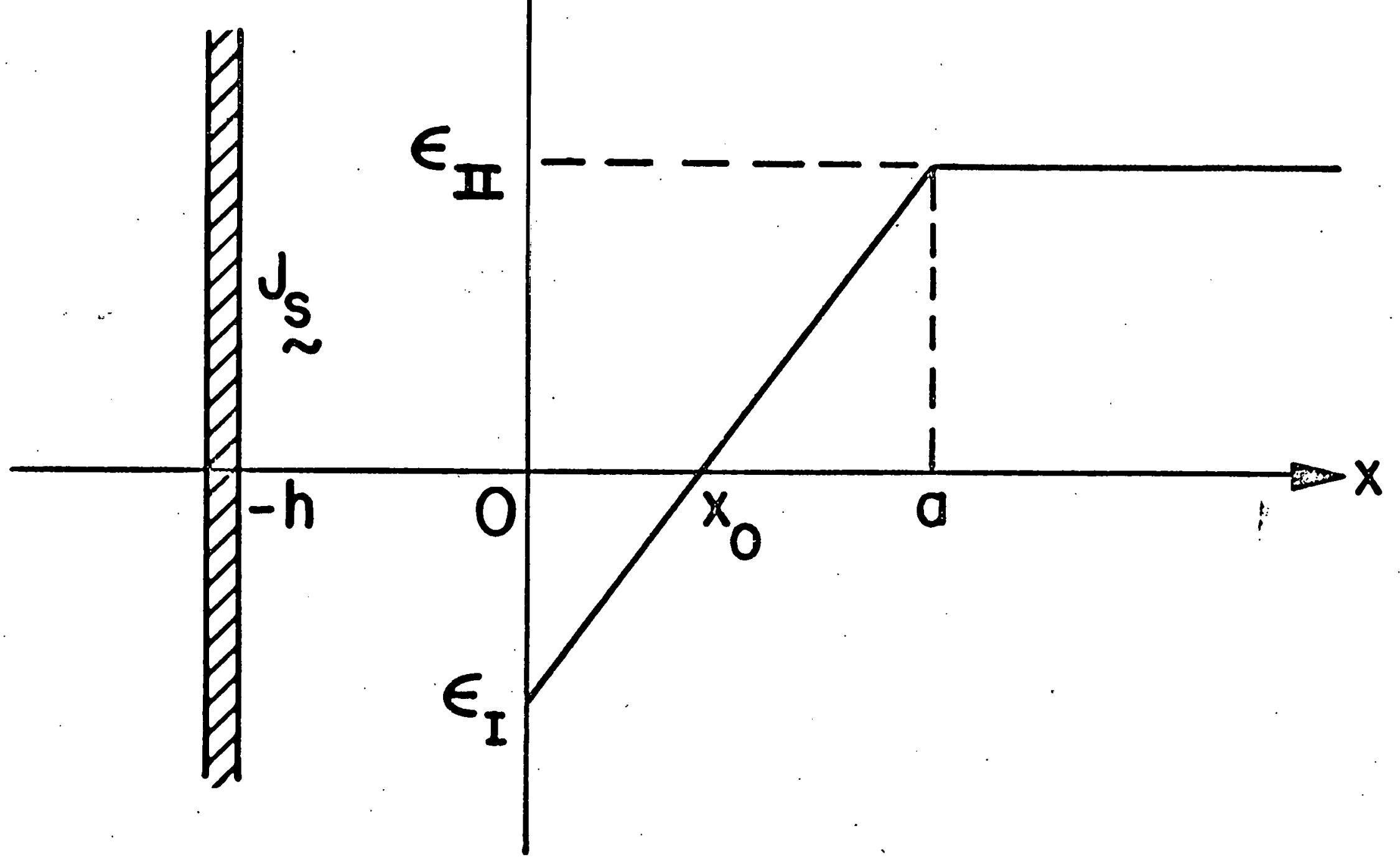




$$
\begin{aligned}
\frac{d W}{d t}= & -\left.\frac{L_{y} L \omega_{0}}{2} \frac{|c|^{2}}{k^{2} \mu_{0}} \frac{d \varepsilon r}{d x}\right|_{x=x_{0}} \\
& \operatorname{Im}\left[\ln \left(x-x_{0}+i \delta\right)\right]_{x_{0}} x_{0}^{+} \\
= & \frac{\omega_{0}}{2} \pi L_{y} L_{z} \frac{|c|^{2}}{k^{2} \mu_{0}}\left|\frac{d \varepsilon r}{d x}\right|_{x=x_{0}},
\end{aligned}
$$

where $\varepsilon_{r}$ is the real part of $\varepsilon$. The above expression shows that the absorption rate is proportional to the gradient of $\varepsilon$ given in Eq. $(7,8)$, and $|\mathrm{C}|^{2}$. C is proportional to the amplitude of the externally applied field intensity. For a design purpose, $C$ should be expressed in terms of driving field. To do this we must solve the boundary value problem of Fig. 7.1 and express $C$ in terms of the current density $\mathrm{J}_{\mathrm{S}}$ of the excitor coil. This process is straightforward but tedious. Referring the process to Chen and Hasegawa (1974), we write down the result here.

$$
\frac{d W}{d t}=2 \pi \omega_{0} \frac{L_{y} L_{z}}{\left|k_{y}\right|} \cdot \frac{1}{\left|k_{y} x_{0}\right|} \frac{1}{|\Gamma|^{2}} \frac{B_{x}^{2}(0)}{\mu_{0}}
$$

where

$$
\begin{aligned}
\Gamma & =\left[I_{0}\left(X_{0}\right)-I_{1}\left(X_{0}\right)\right] D_{1} / D_{2}+K_{0}\left(X_{0}\right)+K_{I}\left(X_{0}\right), \\
D_{1} / D_{2} & =\left[K_{1}\left(X_{0}\right)-K_{0}\left(X_{0}\right)\right] /\left[I_{1}\left(x_{0}\right)+I_{0}\left(x_{0}\right)\right], \\
x_{0} & =\left|k_{y}\right| \varepsilon_{I} / K, \quad x_{a}=\left|k_{y}\right| \varepsilon_{I I} / K,
\end{aligned}
$$




$$
\varepsilon(x) \equiv \begin{cases}\kappa \dot{x}+\varepsilon_{I}, & 0 \leq x \leq a \\ \varepsilon_{I I} & x \geq a\end{cases}
$$

$I_{n}(x)$ and $K_{n}(x)$ are modified Bessel function of the first and the second kind, and $\mathrm{B}_{\mathrm{X}}^{2}(0)$ is the magnetic field induced by $J_{s}$ in vacuum,

$$
\mathrm{B}_{\mathrm{x}}^{2}(0)=\frac{\mu_{0} \mathrm{~J}_{s}}{2} \exp \left[-\left|\mathrm{k}_{\mathrm{y}}\right| \mathrm{h}\right]
$$

It can be shown that if the plasma nonuniformity is weak such that $k / k_{y} \ll 1$, then $\left|x_{0}\right|=\left|k_{y} x_{0}\right| \gg 1,\left|x_{a}\right|=\left|k_{y}\left(a-x_{0}\right)\right|>1$, and $\Gamma \simeq \sqrt{2 \pi}\left(\left|k_{y}\right| x_{0}\right)^{-1 / 2} \exp \left(\left|k_{y}\right| x_{0}\right)$. Then the absorption rate may be written,

$$
\frac{d W}{d t} \simeq \omega_{0} \frac{L_{y} L_{z}}{\left|k_{y}\right|} \frac{B_{x}\left(x_{0}\right)^{2}}{\mu_{0}}
$$

This expression shows that at each cycle of the wave, the magnetic field energy given by $\left|B_{x}\left(x_{0}\right)\right|^{2} / \mu_{0}$ in the volume of $L_{y} L_{z}\left|k_{y}\right|^{-1}$ is absorbed, an indication of a very efficient rate of abisorption.

\subsection{Heating Rate}

To obtain the heating rate of each species in the plasma, we must utilize the kinetic theory. As was shown in section 4.5 , the absorbed energy due to the spatial resonant is converted to the kinetic Alfvern wave. Hence the heating rate of each species can be obtained by the damping rate of the kinetic Alfvén wave by the species (Hasegawa and Chen, 1975, 1976). 
First let us consider the collisional regime. Here the ion heating is dominated by the viscous damping of the transverse component of the wave field. The heating rate is given by

$$
\begin{aligned}
& \mathrm{n}_{0}\left(\mathrm{dT} \mathrm{T}_{\mathrm{i}} / \mathrm{dt}\right)=\frac{1}{2} \operatorname{Re}\left(\mathrm{J} \cdot \mathrm{E}^{*}\right)_{i} \\
& =\left\{\begin{array}{c}
0.7 v_{i i}\left(k_{x} \rho_{i}\right)^{2} \frac{\varepsilon_{0}\left|E_{x}\right|^{2}}{2} \frac{\omega_{p i}^{2}}{\omega_{c i}^{2}} \\
0.9 v_{i i}\left(k_{x} \rho_{i}\right)^{-1} \frac{\varepsilon_{0}\left|E_{x}\right|^{2}}{2} \frac{\omega_{p i}^{2}}{\omega_{c i}^{2}} \text { for } k_{x} \rho_{i}<1,
\end{array} \text { for } k_{x} \rho_{i}>1,\right.
\end{aligned}
$$

where $v_{i i}$ is the ion-ion collision rate. Because $k_{x^{\prime}}\left|E_{x}\right|^{2}$, and $\omega_{\mathrm{pi}}^{2} / \omega_{\mathrm{ci}}^{2}$ are all functions of $\mathrm{x}$, the heating rate varies as a function of the distance away from the mode conversion point. However, the variations tend to cancel among each other and the heating rate remains roughly constant and is given approximately by

$$
\mathrm{n}_{0}\left(\mathrm{dT} \mathrm{T}_{i} / \mathrm{dt}\right) \sim v_{i i}\left[\left|\mathrm{~B}_{\mathrm{y}}\left(\kappa^{-1}\right)\right|^{2} / 2 \mu_{0}\right]
$$

where $B_{Y}\left(k^{-1}\right)$ is the value of the wave magnetic field at $x=k^{-1}$. $B_{y}$ is related to the amplitude of the source field $B_{\text {sy }}$ through the Airy function as shown in section 4.5 ,

$$
\left.\left|B_{y}\right| \sim\left(k \rho_{i}\right)^{-2 / 3}\left[1+\left(T_{e} / T_{i}\right) k_{x}^{2} \rho_{i}^{2}\right]\left|B_{s y}\right|\right),
$$


near the mode conversion region,

$$
\left|B_{y}\right| \sim\left(K \rho_{i}\right)^{-1 / 2}\left[I+\left(T_{e} / T_{i}\right) k_{x}^{2} \rho_{i}^{2}\right]\left|B_{s y}\right|,
$$

away from the mode conversion region (at $x=k^{-1}$ ). If we take a tokamak plasma, $k \sim r^{-1} \sim 10^{-3} \rho_{i}^{-1}$; hence, the $y$ component of the wave magnetic field can be enhanced by a factor of a hundred near the mode conversion point.

The electron heating in a collisional regime is governed by the ohmic dissipation of the field aligned current,

$$
\begin{aligned}
\mathrm{n}_{0}(\mathrm{dT} \mathrm{e} / \mathrm{dt}) & =\frac{1}{2} \operatorname{Re}\left(\mathrm{J}_{z_{z}} \mathrm{E}_{z}^{*}\right) \\
& =\nu_{e i} \frac{\mathrm{T}_{e}}{\mathrm{~T}_{i}} \frac{I}{\lambda_{i}}\left(I-I_{0} e^{-\lambda_{i}}\right)^{2 \frac{\varepsilon_{0}\left|E_{x}\right|^{2}}{2}} \frac{\omega_{\mathrm{p} i}^{2}}{\omega_{\mathrm{C} i}^{2}} \frac{\omega_{0}^{2}}{\mathrm{k}_{z}^{2} v_{\mathrm{Te}}^{2}} .
\end{aligned}
$$

If we compare this heating rate with that of the ions in Eq. (7.18), we see that

$$
\begin{aligned}
\frac{d T_{i} / d t}{d T_{e} / d t} & \sim \frac{\nu_{e i}\left(T_{e} / T_{i}\right)\left(\omega_{0}^{2} / k_{z}^{2} v_{T e}^{2}\right)}{\nu_{i i}} \\
& \sim\left(\frac{m_{i}}{m_{e}}\right)^{1 / 2}\left(\frac{T_{i}}{T_{e}}\right)^{3 / 2} \frac{1}{\beta_{i}} .
\end{aligned}
$$


For most tokamak parameters, this ratio remains of order unity. Hence, in a collisional regime electrons and ions are heated approximately at equal rate. However, electrons are heated in the parallel direction while ions are heated in the perpendicular direction.

In a collisionless regime, the linear heating occurs due to the Landau damping. Hence, particles are heated in the parallel direction, $\mathrm{n}_{0} \mathrm{dT} / \mathrm{dt} \sim \operatorname{Re}\left(\mathrm{J}_{\mathbf{z}} \mathrm{E}_{z}^{*} / 2\right)$. The Landau damping rate for trapped electrons by a local mirror is reduced by the bounce motion. For simplicity, we assume the case of a straight magnetic field. The heating rate for ions in a collisionless regime is then given by

$$
\mathrm{n}_{0} \frac{d \mathrm{~T}_{i}}{\mathrm{dt}}=\omega_{0} \frac{\delta_{i}}{2} \beta_{i}\left(\frac{\mathrm{T}_{\mathrm{e}}}{\mathrm{T}_{i}}\right)^{2} \frac{\mathrm{I}_{0} \mathrm{e}^{-\lambda_{i}}}{\lambda_{i}}\left(1-I_{0} \mathrm{e}^{-\lambda_{i}}\right)^{2} \frac{\varepsilon_{0}\left|E_{x}\right|^{2}}{2} \frac{\omega_{\mathrm{p} i}^{2}}{\omega_{\mathrm{c} i}^{2}},
$$

where

$$
\delta_{i}=2 \sqrt{\pi} \beta_{i}^{-3 / 2} \exp \left(-\beta_{i}^{-1}\right),
$$

and $\beta_{i}$ is defined for the ion pressure, $\beta_{i}=2 v_{\mathrm{Ti}}^{3} / v_{\mathrm{A}}^{2}$ at the resonant point. Other quantities, except $\omega$ are a function of position. Maximum heating is achieved at $\lambda_{i} \sim 1$ 
The heating rate for electrons in a collisionless regime is given by

$$
\mathrm{n}_{0} \frac{d \mathrm{~T}}{\mathrm{dt}}=\omega_{0} \delta_{e} \frac{\mathrm{T}_{\mathrm{e}}}{\mathrm{T}_{i}} \frac{I}{\lambda_{i}}\left(I-I_{0} e^{-\lambda}\right)^{2} \frac{\varepsilon_{0}\left|E_{x}\right|^{2}}{2} \frac{\omega_{p i}^{2}}{\omega_{c i}^{2}},
$$

where $\delta_{e}$ is given by

$$
\delta_{e}=\sqrt{\pi} \beta_{i}^{-1 / 2}\left(T_{i} / T_{e}\right)^{1 / 2}\left(m_{e} / m_{i}\right)^{1 / 2},
$$

In Eqs. (7.24) and (7.26) $\varepsilon_{0}\left|\mathrm{E}_{\mathrm{X}}\right|^{2} \omega_{\mathrm{pi}}^{2} / \omega_{\mathrm{Ci}}^{2}$ can be approximately identified as the wave magnetic field energy $\mathrm{B}_{\mathrm{y}}^{2} / 2 \mu_{0}$.

The ratio of the heating rate for ions and electrons in the collisionless regime becomes

$$
\begin{aligned}
\frac{d \mathrm{~T}_{i} / \mathrm{dt}}{\mathrm{dT} \mathrm{T}_{\mathrm{e}} / \mathrm{dt}} & \sim \frac{(1 / 2) \beta_{i} I_{0} \mathrm{e}^{-\lambda} \mathrm{T}_{\mathrm{T}} / \mathrm{T}_{i} \delta}{\delta_{i}} \\
& \sim\left(\frac{2 \mathrm{~m}_{i}}{\mathrm{~m}_{\mathrm{e}}}\right)^{1 / 2}\left(\frac{\mathrm{T}_{\mathrm{e}}}{\mathrm{T}_{i}}\right)^{3 / 2} \mathrm{e}^{-\beta_{i}^{-1}} \text { for } \lambda_{i} \ll 1 .
\end{aligned}
$$

The factor $\beta_{i}$ on the ion heating rate appears because the ion Landau damping is possible only through the coupling to the ion acoustic wave. This ratio is negligibly small for a low $\beta$ plasma; that is, only electrons can be heated in a low $B$ plasma, However, when $\beta_{i}$ approaches unity, the ratio becomes order unity. For example, if $\beta_{i}=0.2$, $T_{e} / T_{i}=2$ makes this ratio unity and the corresponding value of $\delta_{e}$ becomes 0.13 . Because of the trapped particle 
effect, this value of $\delta_{e}$ is an overestimate. The true damping rate will probably be an order of magnitude less. Let us now consider a nonlinear regime. Because the kinetic Alfvén wave has an enhanced amplitude due to the resonant mode-conversion, even with a reasonably small amplitude of the external source field, nonlinear processes are expected to take place.

As was discussed in Section 6.3 , the kinetic Alfvén wave decays parametrically into another kinetic Alfvén wave and an ion acoustic wave if $T_{e}>T_{i}$. If $T_{e} \simeq T_{i}$, it decays into another kinetic Alfven wave through the nonlinear Landau damping of the ions. In either cases, the ions are heated and the heating rate is roughly proportional to the decay rates given in Egs. $(6.54)$ or (6.57),

$$
\frac{d T_{i}}{d t} \simeq \gamma B_{y}^{2} / 2 \mu_{0}
$$

where $\gamma=\gamma_{D}\left(T_{e} \gg T_{i}\right)$ or $\gamma_{N}\left(T_{e} \simeq T_{i}\right)$, and are ipproximately given by

$$
\gamma_{D} \simeq \frac{\omega_{C i}}{4} \frac{\lambda}{B^{1 / 4}}\left|\frac{B_{y}}{B_{0}}\right|
$$

and

$$
\gamma_{N}=\frac{\omega_{C i}}{8} \frac{\lambda^{2}}{B}\left|\frac{B_{Y}}{B_{0}}\right|^{2} \frac{\omega_{C i}}{\omega_{A}}
$$

and

$$
1>\lambda\left(=k_{1}^{2} \rho_{S}^{2}\right)=\max \left(\lambda_{0}, \lambda_{A^{\prime}} \lambda_{S}\right) .
$$




\subsection{Some Design Examples}

In this section we present some design examples of the application of the kinetic Alfvén wave for supplementary heating of a Tokamak type plasma. As the Ohmic heating saturates at a temperature near $1 \mathrm{kev,} \mathrm{a} \mathrm{supplementary} \mathrm{heating}$ is needed to increase the temperature to a fusion temperature of $10 \mathrm{keV}$. Several methods have been proposed for this purpose, but none is proved conclusively to be the best choice. A reactor Tokamak typically has a dimension of the following size.

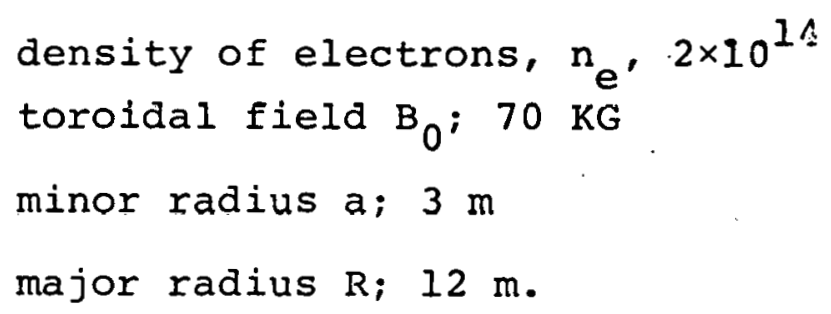

This gives the plasma volume of approximately $2 \times 10^{3} \mathrm{~m}^{3}$ and output power of $3 \mathrm{GW}$. To heat such a plasma within the expected energy containment time of 10 sec., one needs the input power of the supplementary heating of $200 \mathrm{MW}$. If one subtracts heating efficiency, this number indicated that one needs a power source with an efficiency of the order of 50\%. This is rather a severe requirement for a power source with $200 \mathrm{MW}$. The Alfvén wave whose frequency range is on the order of $I \mathrm{MHz}$ is attractive in this respect because a power source of this magnitude and efficiency is currently available. Plasma is heated when the kinetic Alfvén wave is absorbed by the plasma particles. Supplementary heating has meaning only in the collisionless regime where the ohmic 
heating can no longer operate. In this regime, Landau camping or nonlinear wave particle interaction is the important absorption process.

Since the linear Landau damping, which effectively operates only on electrons, is expected to saturate quickly due to the plateau formation of the distribution function, the nonlinear heating is probably the most important process. There, ions can be heated by the nonlinear ion Landau damping. The threshold value of the normalized wave amplitude $\mathrm{B}_{\perp} / \mathrm{B}_{0}$ is given by

$$
v_{i i} \leq 0 \cdot 1 \frac{B_{1}^{2}}{B_{0}^{2}} \frac{\omega_{C i}^{2}}{\omega} \text {. }
$$

Here we have assumed that the electron Landau damping has been saturated by the plateau formation, hence only the ion viscous damping $\nu_{i i} \lambda$ is a dissipative process. The ionion collision frequency $\nu_{i i}$ for $\mathrm{keV}$ plasma with a density of $5 \times 10^{13} \mathrm{~cm}^{-3}$ is approximately $10^{3} \mathrm{sec}^{-1}$. Hence the threshold is given by

$$
B_{\perp}(G) \approx \sqrt{\omega} / 50
$$

If one uses $1 \mathrm{MHz}$, the threshold amplitude of the kinetic Alfvén wave becomes approximately $50 \mathrm{G}$. This is quite a reasonable value if we recall that the amplitude of the kinetic Alfvén wave is enhanced, due to the resonant mode conversion, by a factor of $\left(\rho_{s}\right)^{-1 / 2} \sim 30$, compared with the externally applied field.

If the kinetic Alfvén wave dissipates completely as it propagates through the plasma column, the heating rate 
is given by the absorption rate of the externally applied oscillating field by the resonant absorption. This means that the damping rate per cycle should be larger than the wavelength $\left(\sim \rho_{s}\right)$ over the minor radius of the column, which for the example shown here is $10^{-3}$. The absorption rate is given by

$$
\frac{d W}{d t} \approx V \frac{\stackrel{B}{I}_{\perp}^{e}\left(x_{0}\right)^{2}}{2 \mu_{0}} \omega,
$$

where $V$ is the plasma volume and $B_{\perp}^{e}\left(x_{0}\right)$ is the value of the magnetic field at the location corresponding to the resonant surface in the absence of the plasma. This relation shows that to provide 200 MW to the plasma, one needs only 4 G of oscillating magnetic field. Inside the plasma, this field will be enhanced to about $100 \mathrm{G}$ and will be well beyond the threshold value for the nonlinear Landau damping.

The oscillating RF field with a typical frequency of $1 \mathrm{MHz}$ is launched by a coil placed at the wall helically wound with respect to the toroidal magnetic field. The Ohmic (skin) resistance $R$ of the coil is given by

$$
R=R_{s} \ell / d,
$$

where $d$ and $\ell$ are the width and the length of the coil, and $R_{S}$ is the skin resistance $\sim 10^{-4}$ ohms for a copper and $210^{-3}$ ohms for a tungsten. 
On the other hand, the radiation resistance $R_{r}$ is given by

$$
R_{r} \simeq \frac{\omega_{0}}{2 \pi} \mu_{0} \ell \simeq \omega_{0} L
$$

where $L$ is the coil inductance. Hence the heating efficiency of the coil $\eta$ is given by

$$
n=\frac{1}{1+R / R_{r}}
$$

where

$$
\frac{\mathrm{R}}{\mathrm{R}_{r}} \simeq \frac{1}{2 \pi \times 10^{2} \mathrm{~d}(\mathrm{~m})}
$$

even for a tungsten coil.

If $\mathrm{d} \simeq 0.1 \mathrm{~m}$ is used $\mathrm{R} / \mathrm{R}_{\mathrm{r}} \ll 1$ and $\eta \simeq 1$. The $Q$ value of the coil itself is given by $\omega_{0} L / R \sim 2 \pi \times 10^{2} \mathrm{~d}$. The $Q$ value of the coil including the radiation impedance is order unity because of the high efficiency of the resonant absorption.

The induced terminal voltage $\mathrm{V}$ of the coil is given by

$$
V=\omega_{0} L I
$$

To produce $10 \mathrm{G}$ amplitude of $\tilde{B}_{\perp}^{e}\left(\mathrm{X}_{0}\right)$ at $1 \mathrm{MHz}$ with a coil of $1 \mathrm{~m}$ length produce $\mathrm{V} \simeq 100$ Volt. 
The excitation by a coil has a merit in that it can be designed such that the magnetosonic wave is made cut off by choosing $k_{y} v_{A}\left(=k_{\theta} v_{A}\right)>$ whereby one can suppress the unnecessary compressional motion of the plasma. But it has demerits that originate from the installation of a large coil inside the reactor chamber. An alternative method is to use a waveguide, or a smaller antenna coupling to excite a magnetosonic wave which is resonantly absorbed at the Alfvén layer as shown by Eq. (7.10). If a plasma can tolerate the compressional motion of the magnetosonic wave, this method can eliminate the problem of the large antenna. (Ott et al., 1978; Kearney and Perkins, 1978). 
- $198-$

LAUSANNE $\theta$ PINCH

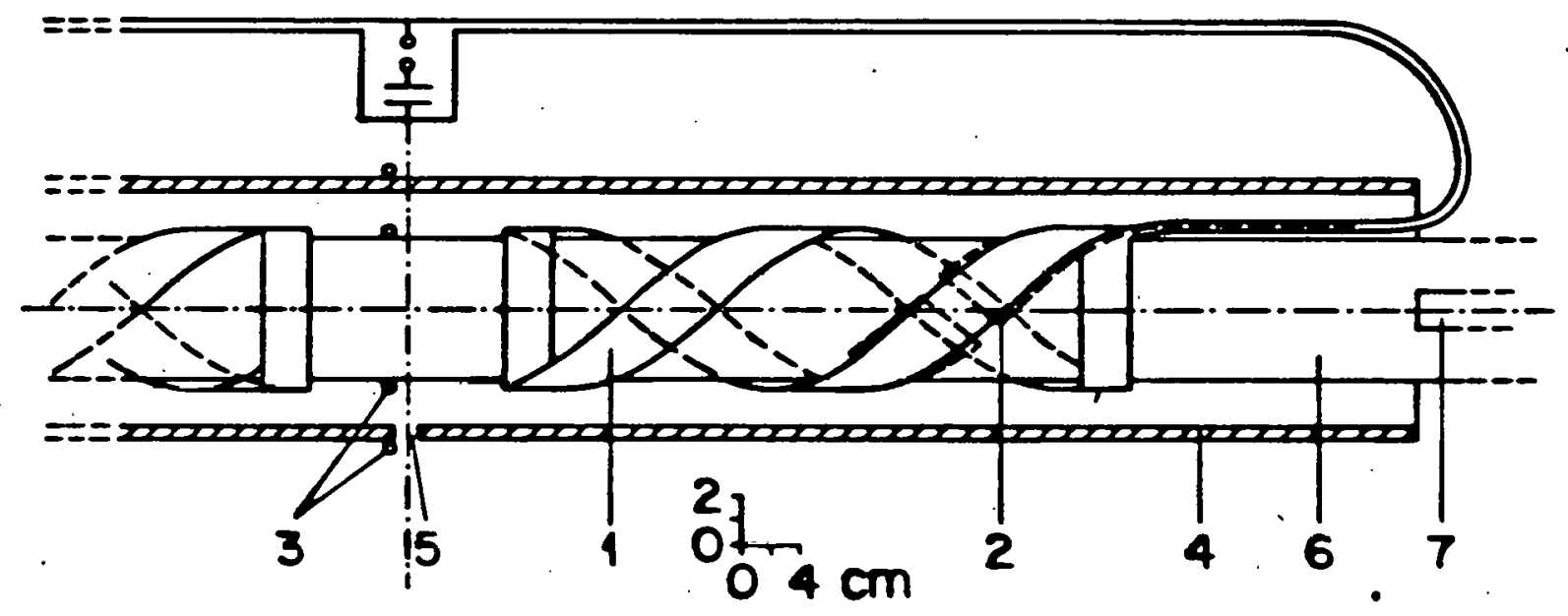

7.2 


\subsection{Experimental Results}

We introduce here four major experiments of Alfvén

wave heating. (1) Theta pinch plasma in Lausanne, (2)

Stellarator plasma in Wisconsin, (3) Stellarator plasma in Suhumi and (4) Heliotron plasma in Kyoto. In all these experiments, an efficient absorption and heating have been observed, however, there seem to exist discrepancies in the existence of enhanced loss of plasma.

Here we introduce a brief summary of experimental results of these machines in this order.

Theta pinch.

Alfvén wave heating on linear theta pinch plasma has been studied by Keller and Pochelon (1978). The pinch configuration and the helical launching structure are shown in Fig. 7.2. The theta coil has a length of $142 \mathrm{~cm}$ and an inner diameter of $9 \mathrm{~cm}$. The main field reaches $16 \mathrm{~kg}$ in $3.8 \mu \mathrm{s}$ at which time it is crowbarred. The quartz discharge tube has an inner diameter of $5.2 \mathrm{~cm}$. Two electrodes, $1.2 \mathrm{~cm}$ in diameter, are $142 \mathrm{~cm}$ apart. The typical plasma parameters are: percentage of ionization, $75 \%$; mean plasma radius, $0.76 \mathrm{~cm}$; mean electron density, $2 \times 10^{16} \mathrm{~cm}^{-3}$; maximum plasma temperature (before heating), $40 \mathrm{eV}$. The helical launching structure has an $m=1$ configuration. The energy source is a $45 \mathrm{kV}, 0.1 \mu \mathrm{F}$ low inductor condenser. Coupled with the coil system, the ringing frequency is $1 \mathrm{MHz}$. The temperature and heating power are measured by diamagnetic probe. 
$-200-$

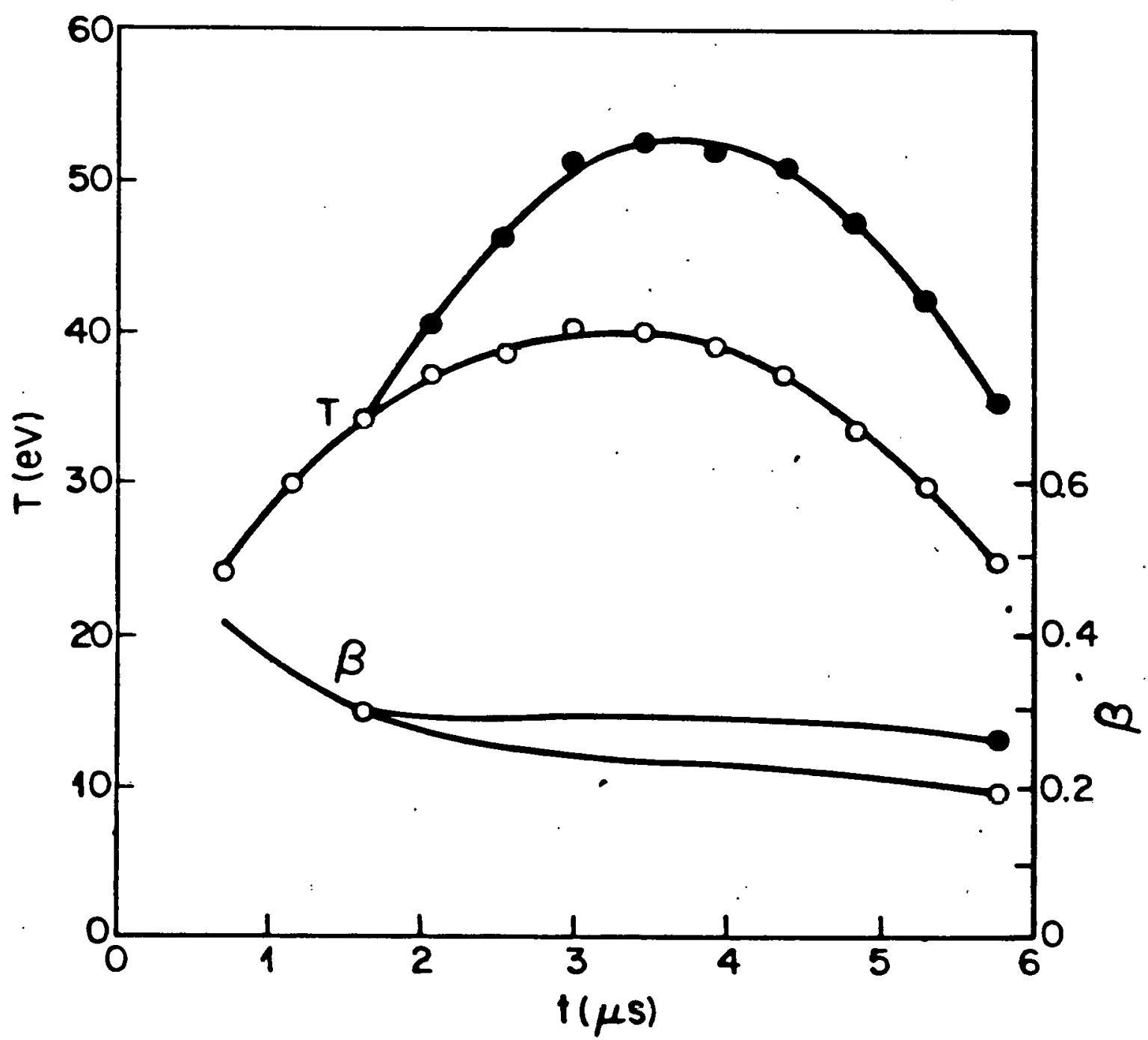

7.3 


\section{PROTO-CLEO}

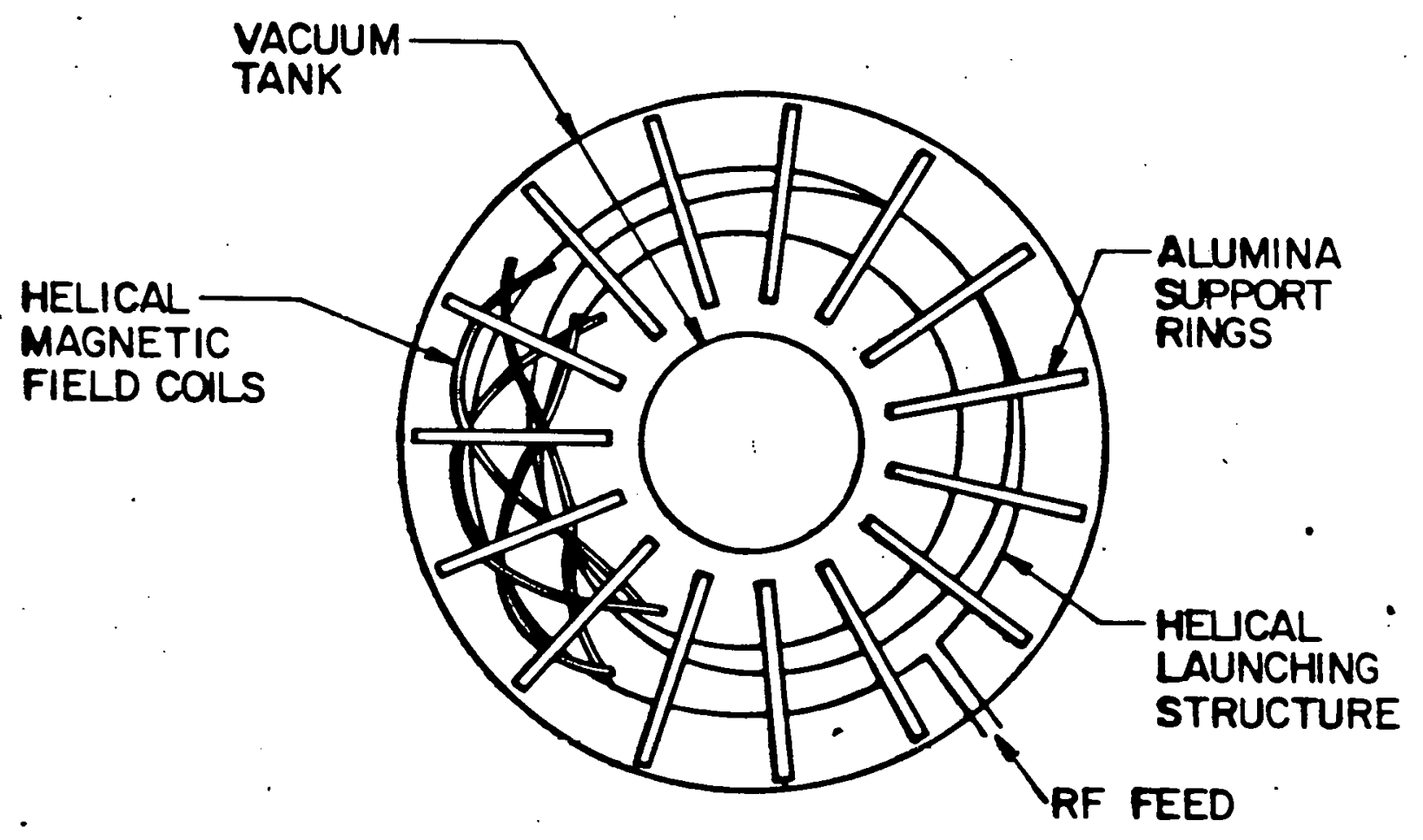

7,4 


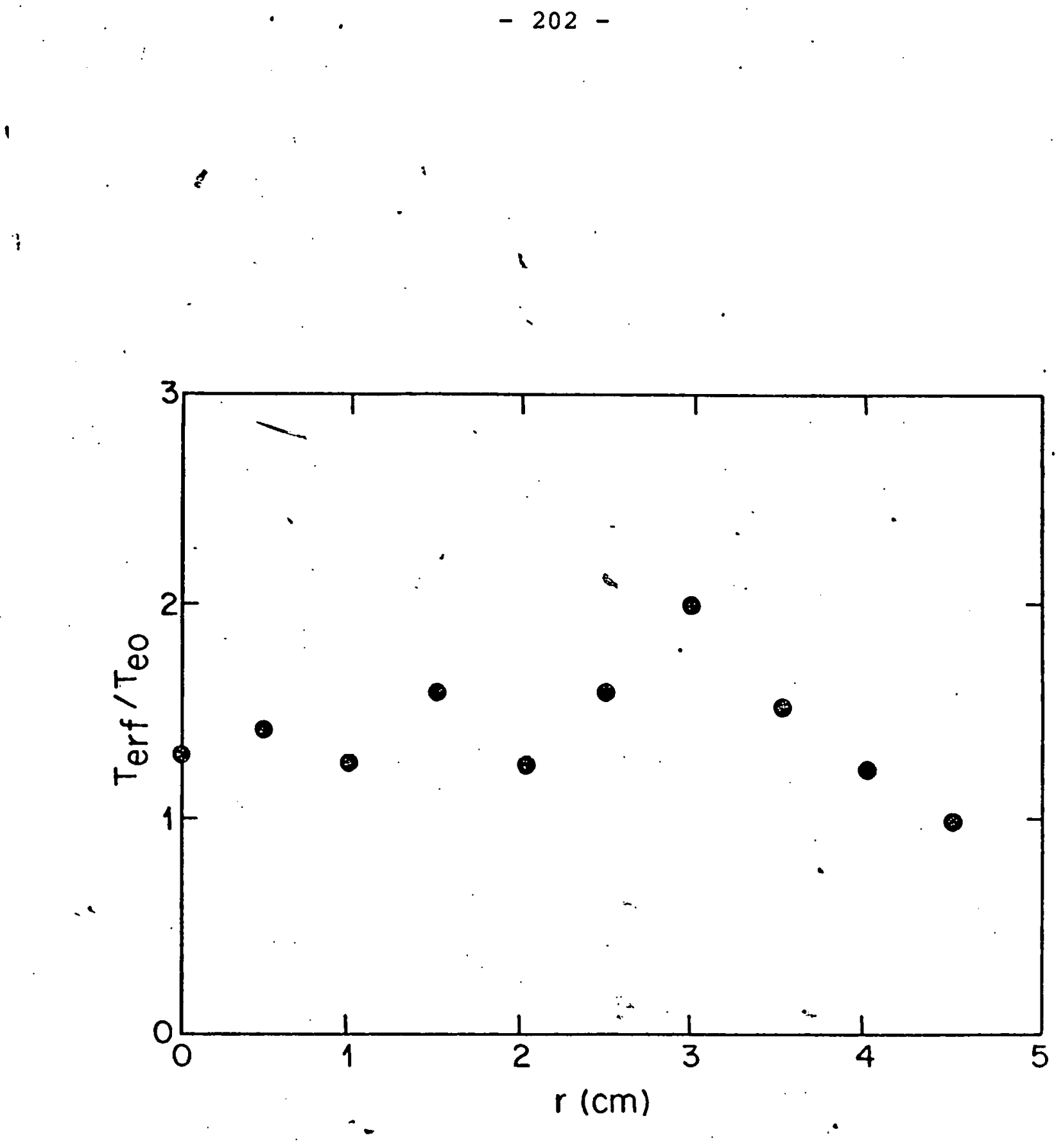


Figure 7.3 shows the increase of temperature (T) and plasma beta $(\beta)$ as the functional time. The heating efficiency deduced from the experimental data is approximately $50 \%$. Evidence of resonant absorption is observed. Proto-cleo.

Alfuén wave heating on proto-cleo plasma at Wisconsin has been reported by Golovato et al. (1976). The proto-cleo is operated as an $\ell=3,7$ field period stellarator, with a major radius of $0.4 \mathrm{~m}$ and an average plasma minor radius of $5 \mathrm{~cm}$. The plasma density is approximately $10^{12} \mathrm{~cm}^{-3}$ and the electron temperature of the order of $10-20 \mathrm{eV}$. The poloidal field is $3 \mathrm{kG}$ and the energy confinement time is 1 ms. The experimental setup is shown in Fig. 7.4. The r.f. coil is the helical winding that goes three times around the major axis of the torus in going once around the minor axis. It is inside the higher pitched confining field windings. An up to $200 \mathrm{~kW}$ generator operating at pulse length of $1 \mathrm{~ms}$ was used to launch r.f. Figure 7.5 is the plot of the radial elcctron temperature profile immediately after r.f. was cut off, normalized to the temperature profile at the same time without the presence of $r . f$. Notice that several peaks are observed. These peaks roughly correspond to the spacial resonant surfaces assuming one dimensional theory. Both the electron and ion temperatures appear to be doubled. One unique feature of this experiment 
- $204-$

RO-2 STELLARATOR

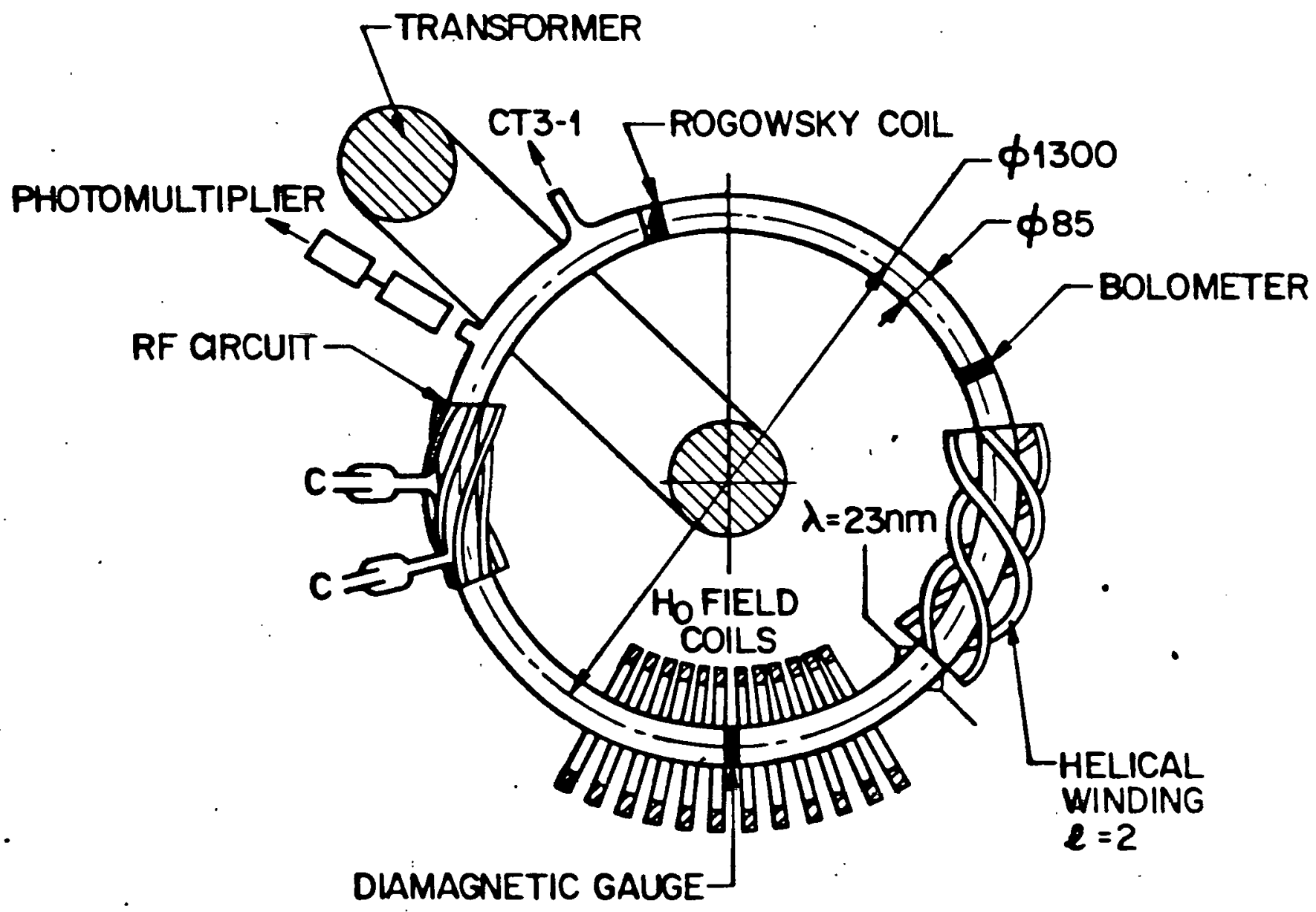

7.6 


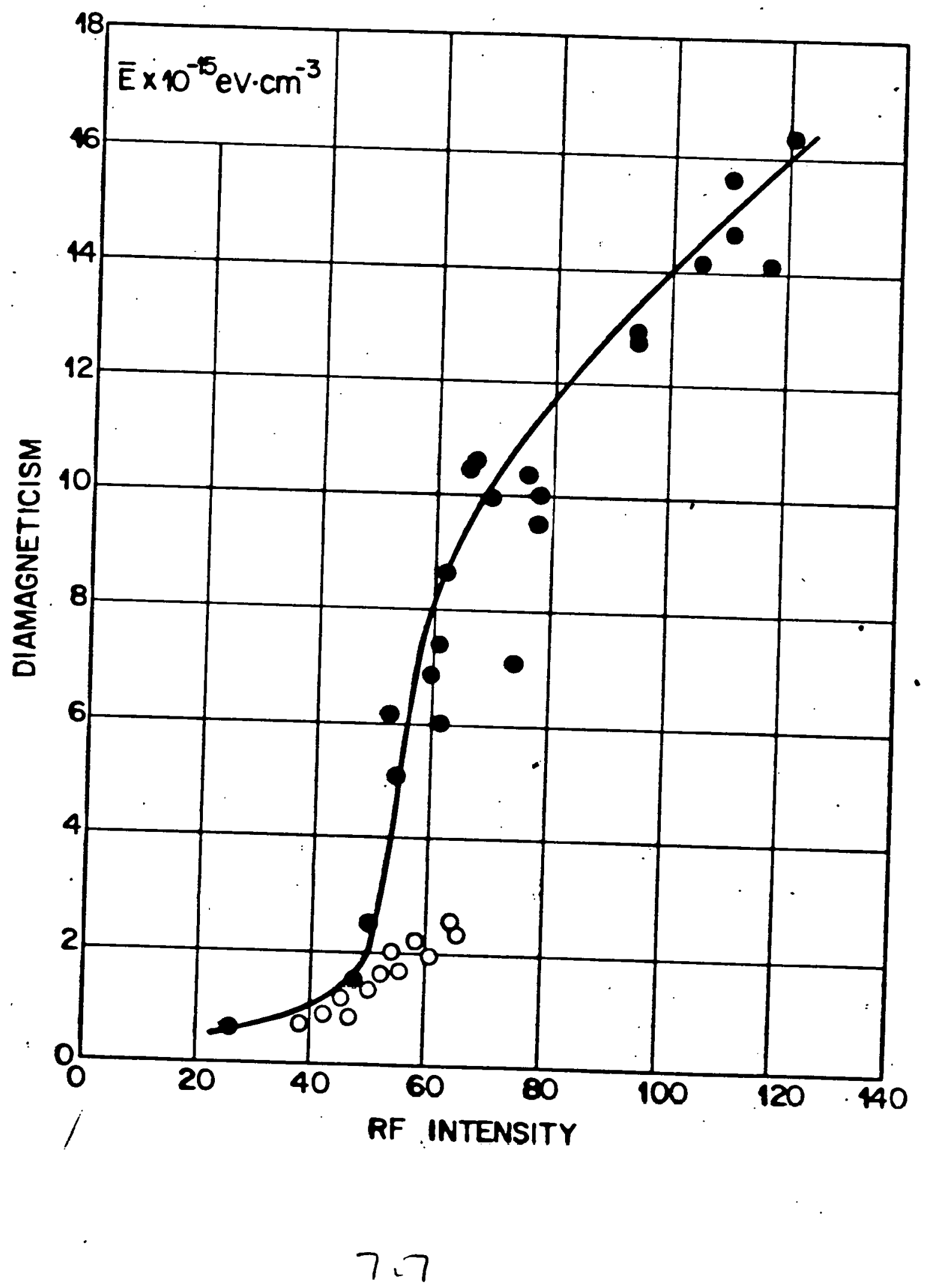


is the observation of enhanced transport due to the applied r.f. field. The anomalous transport varies approximately linearly with respect to the amplitude of $r . f$. and is believed to be caused by magnetic island formation.

RO-2.

Alfvén wave heating experiment on RO-2 at Suhumi is interesting because of the high intensity r.f. field (Demirhanov, et al. 1977). The schematic diagram of RO-2 and its coupling coil is shown in Fig. 7.6 .

Efficient heating is found to take place when the plasma density is made larger than $5 \times 10^{13} \mathrm{~cm}^{-3}$, at which density the Alfvén wave can be coupled into the plasma. Figure 7.7 shows the clear nonlinear aspect of the heating as a function of r.f. magnetic field intensity. It is also interesting that the temperature reached almost 20 times of the initial temperature. In actual numbers, the ion temperature increases from $20 \mathrm{eV}$ to $250 \mathrm{eV}$. The nonlinear threshold of the heating takes place at the r.f. magnetic field amplitude of about 50 Gauss. One remarkable aspect of this experiment is that the fluctuating field observed by loop voltage has disappeared and better confinement is achieved when the Alfvén wave is coupled in the higher density phase of the discharge. The better confinement in the presence of large amplitude Alfvén wave may be understood in terms of the negative ponderomotive effect as was discussed in Section 6.5. Unlike 
1

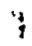

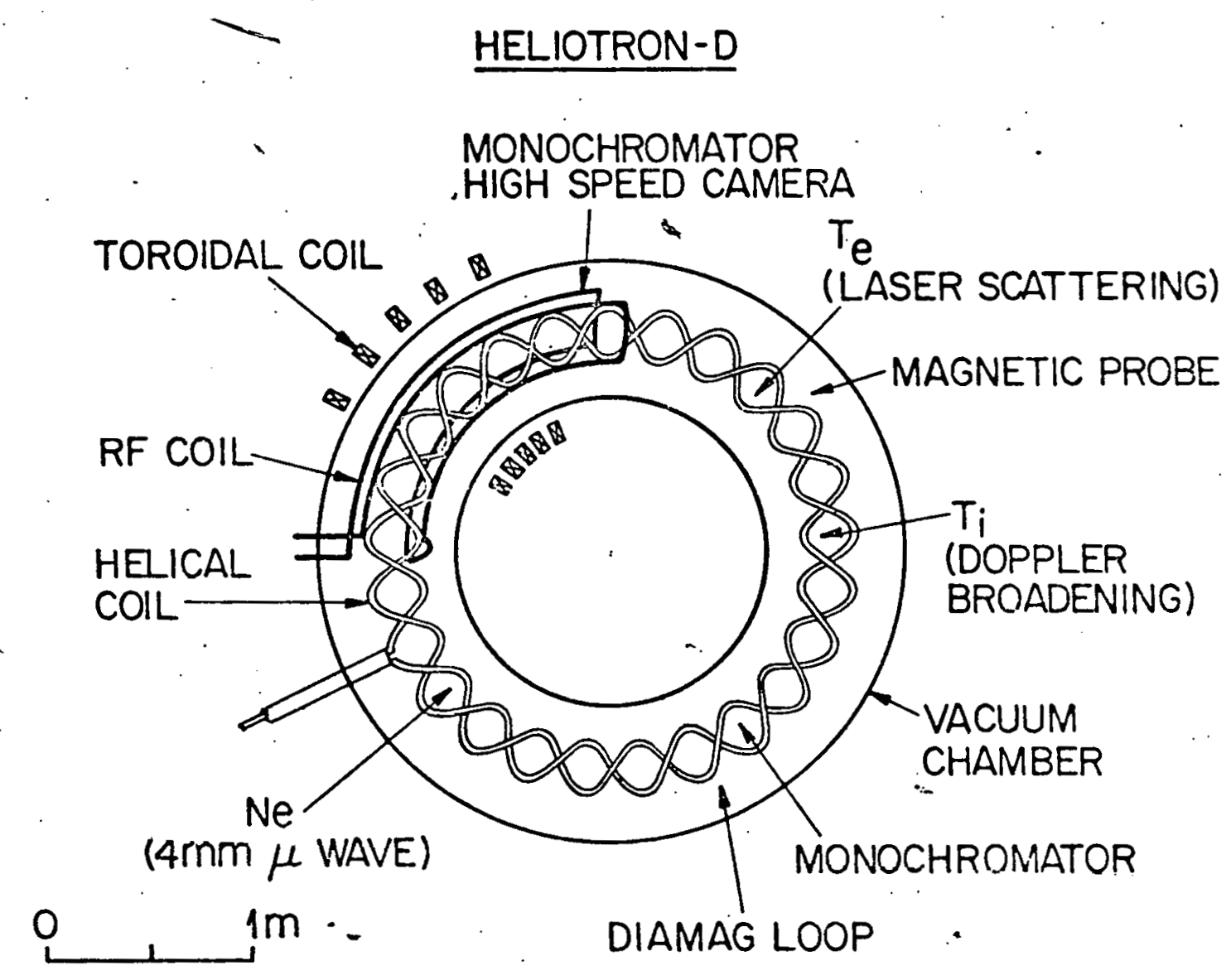




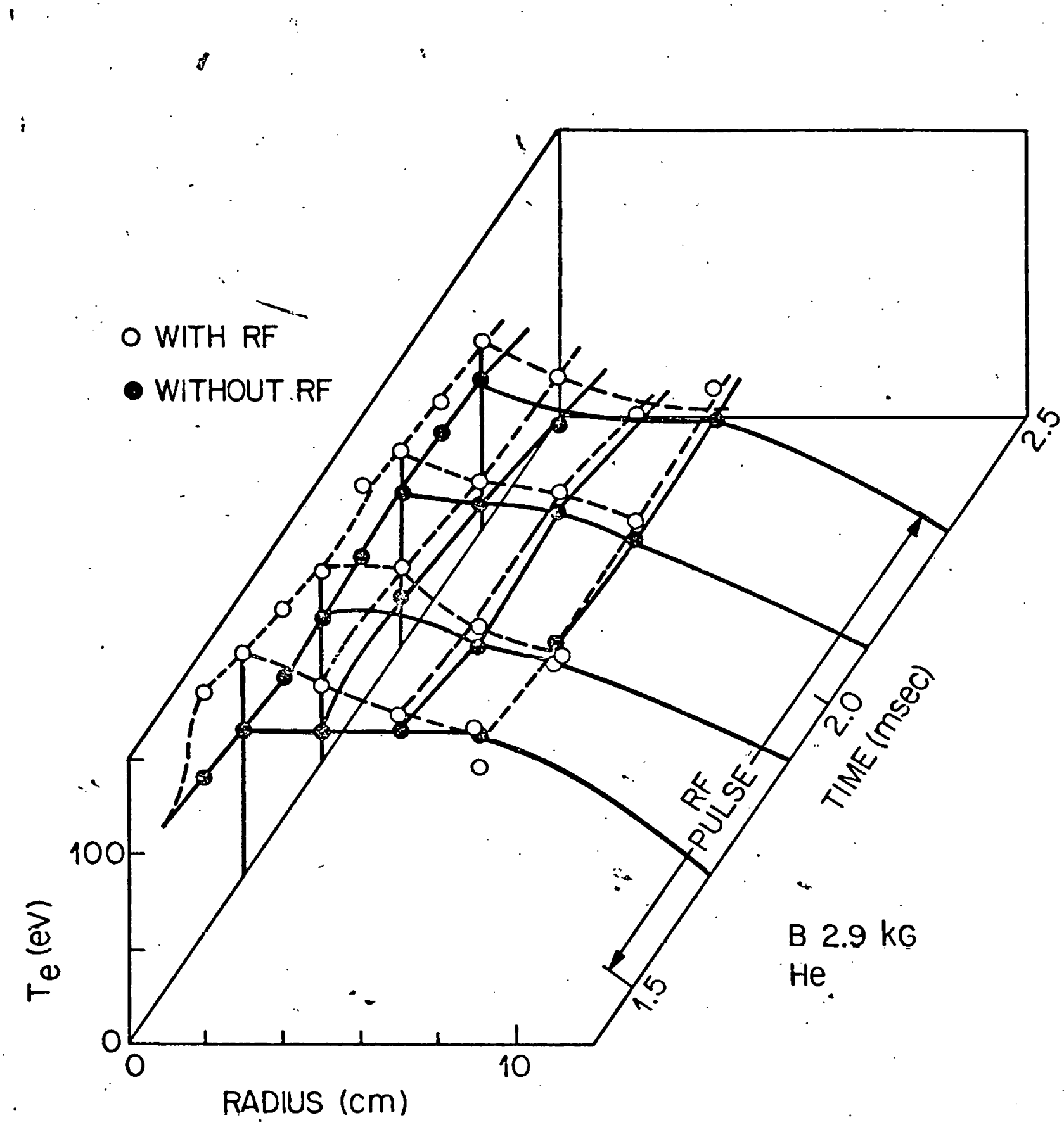


$-209-$

1

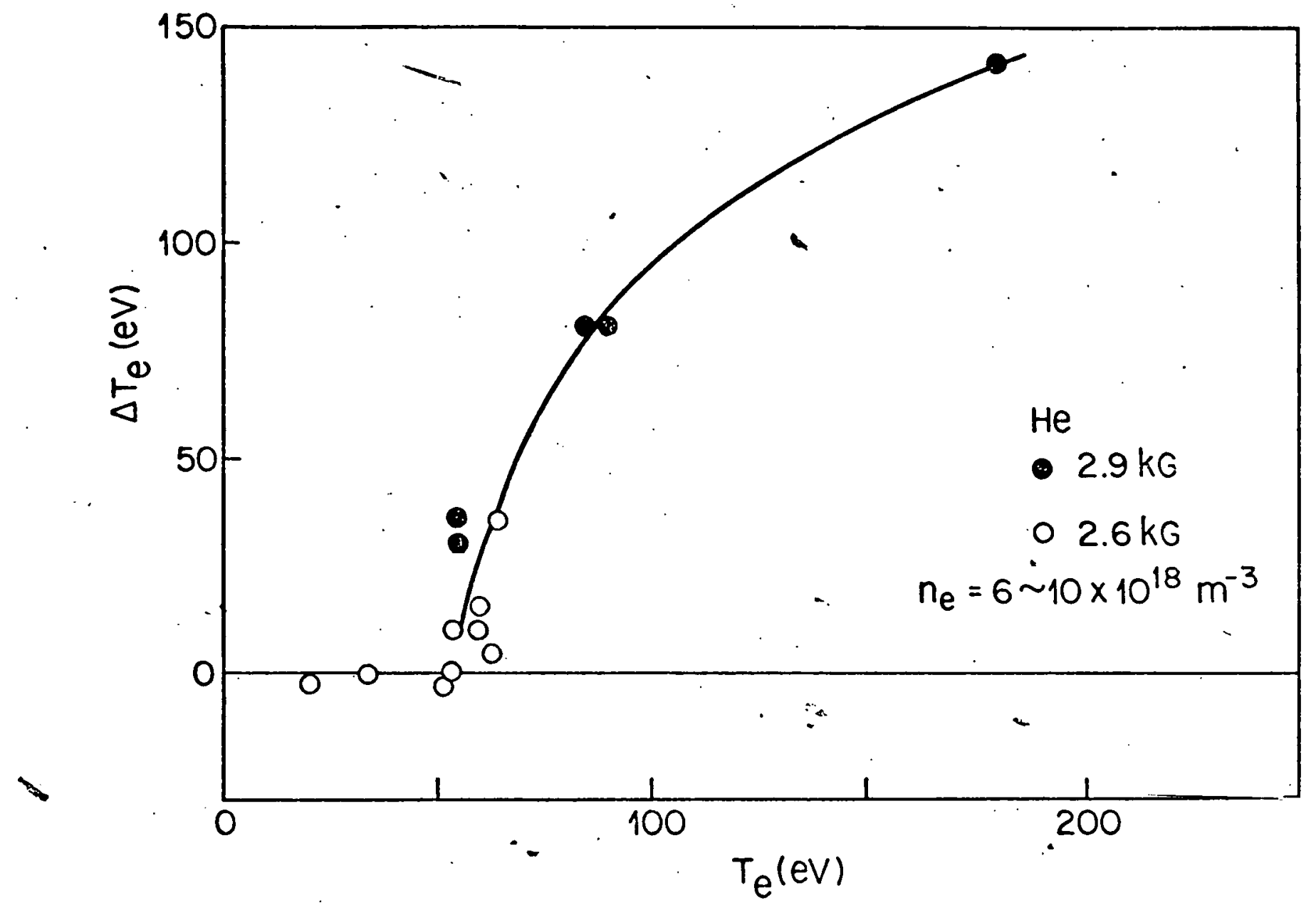

7.10 
the case of electrostatic ponderomotive force, magnetic ponderomotive force tends to attract plasma into high intensity region of r.f. field. If this is proved to be the case, the Alfvén wave heating has a very attractive feature in that the applied r.f. field works to heat as well confine the plasma.

Heliotron-D

Alfvén wave heating of Heliotron-D has been reported by obiki et al. (1977). The machine has a major radius of $1.1 \mathrm{~m}$, minor radius of $10 \mathrm{~cm}$, with the toroidal field of 2-3 kG, electron density ranging between $3 \times 10^{12}-3 \times 10^{13} \mathrm{~cm}^{-3}$ The plasma is Ohmically heated before the Alfvén wave heating is applied. A schematic diagram of the Heliotron is shown in Fig. 7.8. The initial experimental data tend to show a successful heating but poor confinement of electron heat. However, most recent results seem to indicate a good heating and good confinement as shown in Fig. 7.9. The improvement in confinement is achieved by better shielding of the $r . f$. coil. One remarkable result of this experiment is in the clear threshold of efficient heating as the function of initial electron temperature as shown in Fig. 7.10. As was shown in Section 7.3 , the heating occurs due to the dissipation of the kinetic Alfvén wave which is excited at the resonant surface. However, the kinetic Alfvén wave can exist only when the electron thermal speed is larger 
than the Alfvén speed at the resonant surface. Otherwise, the kinetic Alfvern wave cannot exist hence the mode conversion cannot take place. In addition when the electron thermal speed is larger than the Alfvern speed, electron Landau damping and the consequent heating of electrons can take place. This threshold can be seen in Fig. 7.10; the temperature increases only when this condition is satisfied. If the kinetic Alfvern wave cannot be excited, the heating takes place only locally at the resonant surface. Therefore, having an initially large enough electron temperature such that its thermal speed is larger than the Alfvén speed is essential for a bulk heating. Among three toroidal experiments introduced here, the wisconsin results fall into the case of no kinetic Alfvén wave excitation. The enhanced plasma transport which seems to exist only in the Wisconsin experiment may be related to this point. 
REFERENCES FOR CHAPTER VII.

Chen, Ifila and Hasegawa, A., Plasma heating by spatial resonance of Alfvén wave, Phys. Fluids 17, 1399-403 (1974)

Demirkhanov, R. A., Kirov, A. G., Ilinsky, S. E., Mayburov,. V. B., Onischenko, V. V., Tarasevich, A. F., Kadish, E. Y. and Malich, L. Y., Heating of plasma in toroidal trap by helical multiple.h.f. magnetic field, Plasma Physics and Controlled Fusion Res. (IAEA, Vienna, 1977 ) 3,31 .

Golovata, S. N., Shohet, J. L. and Tataronis, J. A., Alfvén wave heating in the Proto-Cleo stellarator, Phys. Rev. Lett. 37, 1272 (1976).

Grossmann, W. and Tataronis, J., Decay of MHD wave by phase mixing II, theta pinch in cylindrical geometry, $Z$. Phys. 261, 217 (1973).

Hasegawa, A. and Chen, L., Plasma heating by Alfvén wave phase mixing, Phys: Rev. Lett. 32, 45! (1974).

Hasegawa, A. and Chen, L., Kinetic process of plasma heating due to Alfvën wave excllaliur, Fhys. Rov. Lett. 35,370 (1975).

Hasegawa, A. and Chen, L., Kinetic Processes in plasma heating by resonant mode conversion of Alfvén wave, Phys. Fluids 19,1924 (1976).

Kearney, C. F. F. and Perkins, F. W., Alfvén resonance heating via magnetosonic modes in large Tokamaks, Plassma Physics Laboratory PPPL-1471 (1978).

Keller, R. and Pochelon, A., Alfvén wave heating of a theta pinch, Nucl. Fusion 18 (1978) to be published. 


\section{REFERENCES FOR CHAPTER VII.}

Obiki, T., Mutoh, T., Adachi, S., Sasaki, A., Iiyoshi, A., and Uo, K., Alfvén wave heating experiment in the Heliotron-D, Phys. Rev. Lett. 39, 81? (1977), also IAEA-CN-37-L-1 (.1978).

Ott, E., Wersinger, J.-M. and Bonoli, P. T., Theory of plasma heating by magnetosonic cavity mode absorption, Laboratory of Plasma Studies, LPS 236, Cornell University, 1978.

Pochelon, A. and Keller, R., Alfvén wave heating experiment of a high beta plasma, Helvetica Physica Acta $\underline{50}$, $172(1977)$. 


\section{OTHER REFERENCES FOR CHAPTER VII.}

Alazraki, G., Couturier, P., Solar wind acceleration caused by the gradient of Alfven wave pressure, Astron. and Astrophys. 13, 380-9 (1971).

Hasegawa, A. and Chen, L., Parametric decay of 'kinetic Alfven wave' and its application to plasma heating, Phys. Rev. Lett. 36, 1263-5 (1976).

Hollweg, J. V., Alfvenic acceleration of solar wind helium and related phenomena. I. Theory. J. Geophys. Res. 79, $1357-63(1974)$.

Lacombe, C., Contribution of the non-linear Landau damping of Alf'vên waves to the heating of the solar wind protons, Astron. and Astrophys. 48, 11-17 (1976).

Piddington, J. H., Solar atmosphere heating, Sol. Phys. (Netherlands) 33, 363-73 (1973).

Tataronis, J. A., Grossmann, W., On Alfvén wave heating and transit time magnetic pumping in the guiding-centre model of a plasma, Nucl. Fusion 16, 667-18 (1976). Uchida, Y., Kaburaki, 0., Excess heating of corona and chromosphere above magnetic regions by non-linear Alfvén waves, Sn.t. Phys. (Netherlands) 35, 451-66 (1974).

Wentzel, D. G., Coronal heating by Alfvén waves, Sol. Phys. (Netherlands) 39, 129-40 (1974). 


\section{LIST OF SYMBOLS}

B

C

$\mathrm{C}_{\mathrm{s}}$

D

$D(\omega, k)$

E

$E_{k}$

e;e

F

$f(x, v, t)$

Im

$I_{n}$

i

$\mathrm{J}$

$\mathrm{J}_{\mathrm{n}}$

$\mathrm{K}_{\mathrm{n}}$

k

$\mathrm{k}_{\mathrm{D}}$ magnetic field vector

speed of light in vacuum; $2.998 \times 10^{8} \mathrm{~m} / \mathrm{sec}$

ion sound speed $v_{T e}\left(m_{e} / m_{i}\right)^{1 / 2}$

diffusion constant

dispersion relation; $D(\omega, k)=0$

electric field vector

Fourier amplitude of an electric field vector with the wave vector $k$.

electron charge; $1.602 \times 10^{-19}$ coulomb; base of natural logarithm

force

distribution function in $v-x$ phase space

imaginary part

modified Bessel function of the first kind

$\sqrt{-1}$

current density

Bessel function of the first kind

modified Bessel function of the second kind

wave vector

Debye wave number; $\omega_{\mathrm{p}} / \mathrm{v}_{\mathrm{T}}$ 


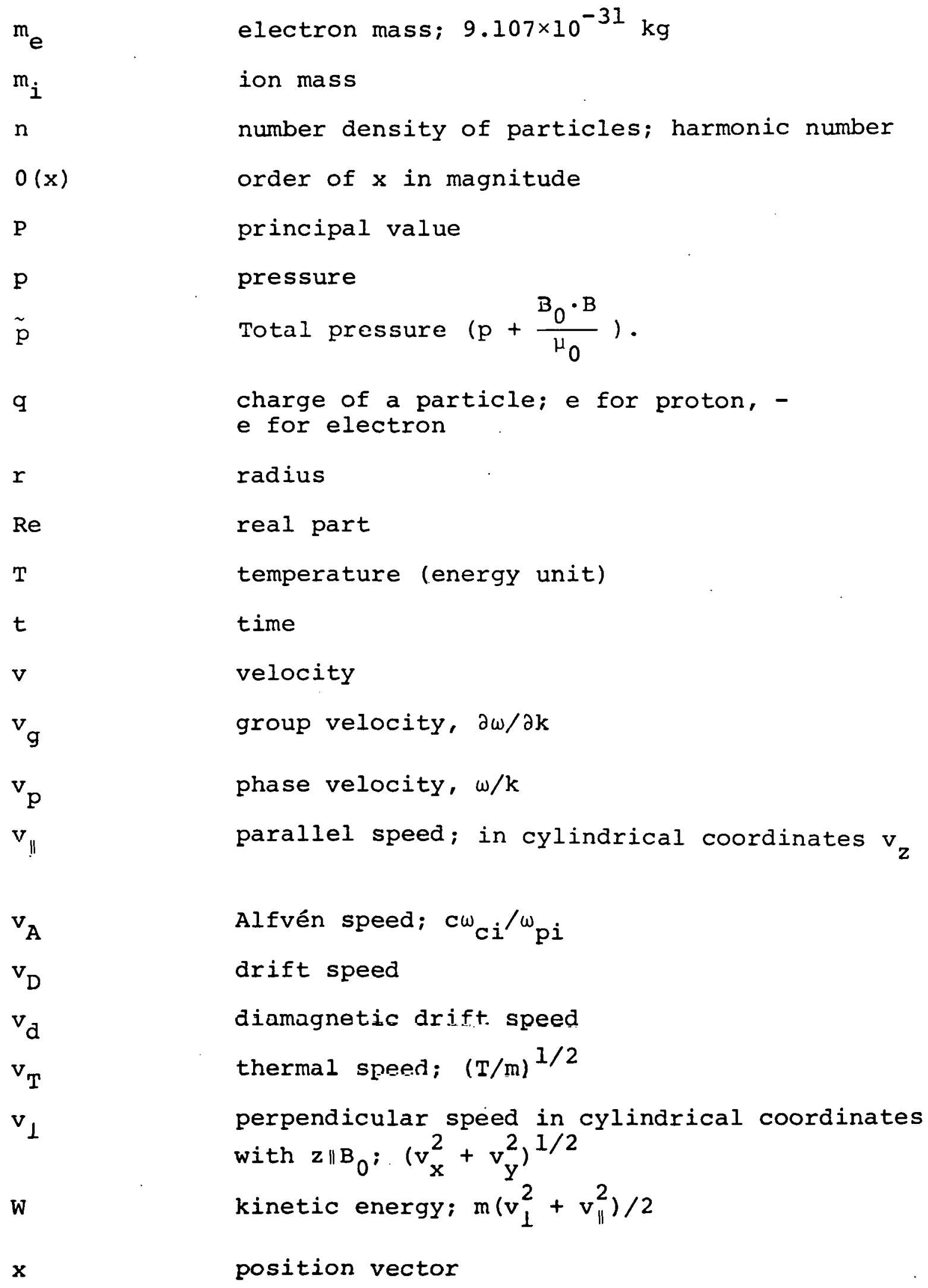




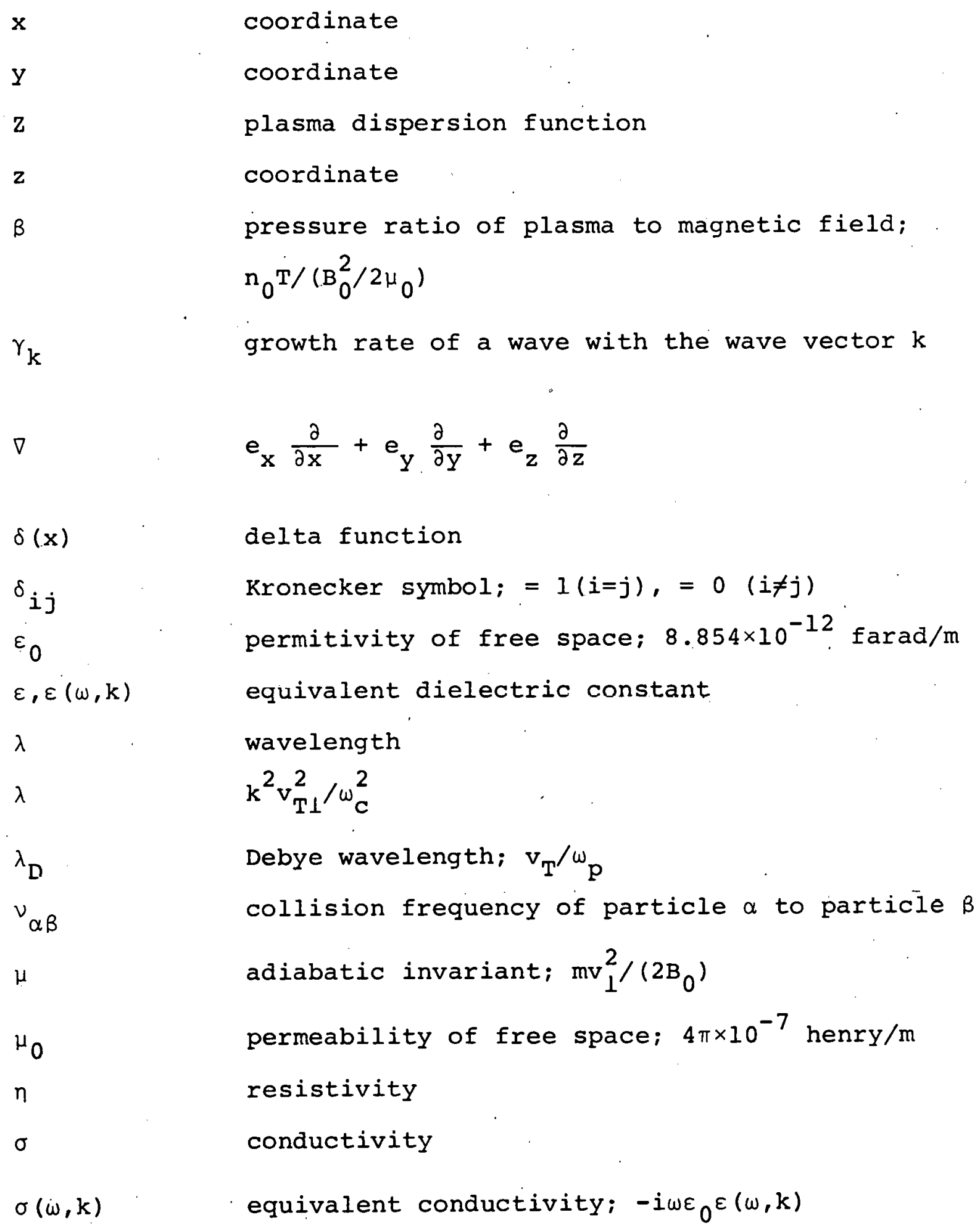


K

p

$\tau$

$\tau$

$\phi$

$\Phi$

$\omega_{k}$

$\omega_{p}$

${ }^{\omega} \mathrm{C}$

$\omega^{\star}$

$\xi$

$\xi$

$<>$

Superscript*

Subscript e

Subscript

Subscript i

Subscript $p$

Subscript $r$

Subscript 0

Subscript $\perp$

Subscript measure of density gradient; $-\nabla\left(\ell n n_{0}\right)$

charge density

Larmor radius

mean free time

reduced time

electrostatic potential

amplitude of a modulated wave

solution for $\omega$ for a given value of $k$ of a linear dispersion relation

plasma (angular) frequency; $\left(e^{2} n_{0} / \varepsilon_{0} m\right)^{1 / 2}$

cyclotron (angular) frequency; $\ell \mathrm{B}_{0} / \mathrm{m}$

drift wave (angular) frequency; $k_{1} v_{T}^{2} \nabla\left(\ell n n_{0}\right) / \omega_{C}$ reduced spatial coordinate vector

plasma displacement; $\partial \xi / \partial t=v$

ensemble average

complex conjugate

electron

ion (proton)

imaginary part

plasma

real part

unperturbed (dc) quantities

perpendicular to the magnetic field

parallel to the magnetic field 


\section{MHD Equations}

$$
\begin{aligned}
& \frac{\partial n}{\partial t}+\nabla \cdot(\underset{\sim}{v})=0, \\
& m_{i} n \frac{d \underset{\sim}{v}}{d t}=\underset{\sim}{J \times \underset{\sim}{B}-\nabla p}, \\
& \underset{\sim}{E}+\underset{\sim}{v} \times \underset{\sim}{B}=\eta_{\sim}^{J}, \\
& \frac{d p}{d n}=\frac{5}{3} \frac{p}{n}, \\
& \nabla \times \underset{\sim}{E}=-\frac{\partial \tilde{B}}{\partial \tilde{t}}, \\
& \nabla \times \underset{\sim}{\mathrm{B}}=\mu_{\mathrm{O}}^{\mathrm{J}}, \\
& \nabla \cdot \underset{\sim}{B}=0
\end{aligned}
$$

II. Vlasov-Maxwell's Equations

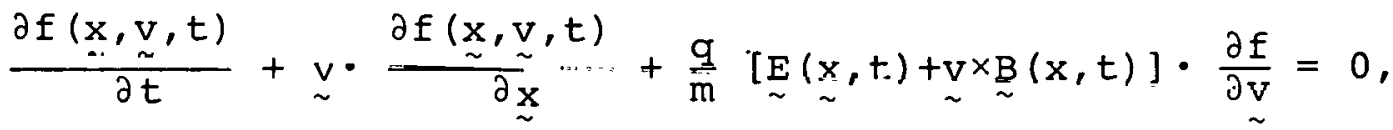

$$
\begin{aligned}
& \nabla \times \underset{\sim}{E}=-\frac{\partial \underline{\tilde{B}}}{\partial t},
\end{aligned}
$$

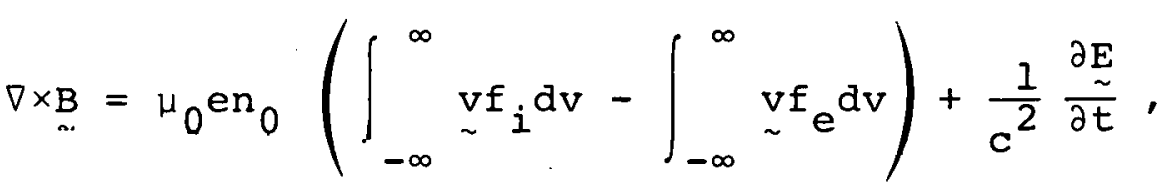

$$
\begin{aligned}
& \nabla \cdot \underset{\sim}{\mathrm{B}}=0 \text {, }
\end{aligned}
$$




$$
\nabla \cdot \underset{\sim}{E}=\frac{e n_{0}}{\varepsilon_{0}} \int_{-\infty}^{\infty} f_{i} d \underline{\sim}-\int_{-\infty}^{\infty} f_{e} \underset{\sim}{d v} .
$$

III. Drift Kinetic Equations

$$
\begin{aligned}
& \frac{\partial f_{D}}{\partial t}+\nabla \cdot\left({\underset{\sim}{D}}_{D} f_{D}\right)+\frac{\partial}{\partial v_{\|}}\left(\frac{F_{\|}}{m} f_{D}\right)=0, \\
& f_{D}=f_{D}\left(v_{\|}, \mu \underset{\sim}{x}, t\right), \\
& \underset{\sim D}{v_{D}}=\underset{\sim}{v_{D}}+\underset{\sim}{v_{D} \|}, \\
& \underset{\sim}{\mathrm{V} \|}=\mathrm{v}_{\|} \underset{\sim}{\mathrm{B} / \mathrm{B}}, \\
& v_{D \perp}=v_{\sim}+\frac{\underset{\sim}{F} \times \frac{B}{\sim}}{q B^{2}}, \\
& \mathrm{v}_{\mathrm{E}}=\frac{\underset{\sim}{\left(\mathrm{E}+v_{\|} \times \sim_{\sim}\right) \times \underset{\sim}{B}}}{\mathrm{~B}^{2}}, \\
& \underset{\sim}{F}=-\mu \nabla_{\perp} B-\frac{m v_{!}^{2}}{R} \underset{\sim}{\hat{R}}-m \frac{d v_{E}}{d t}+\underset{\sim}{m}, \\
& \underset{\sim}{\mathrm{F}}=-\mu \nabla_{\|} \mathrm{B}+\underset{\sim}{\mathrm{qE}} \|
\end{aligned}
$$


IV. Plasma Dielectric Tensor

$$
\begin{aligned}
& \underset{\sim}{k}\left(\underset{\sim}{k} \cdot \underset{\sim}{E_{1}}\right)-k^{2}{\underset{\sim}{E}}_{1}+\frac{\omega^{2}}{c^{2}} \underset{\sim}{(I+E)} \cdot \underset{\sim}{\mathbb{E}}{ }_{1}=0, \\
& \left.\stackrel{\leftrightarrow}{\varepsilon}=-\sum_{\text {species }} \frac{\omega_{p}^{2}}{\omega^{2}} \int_{I}^{\stackrel{\leftrightarrow}{I}}+\sum_{n=-\infty}^{\infty} \int \underset{\sim}{d v} \frac{k_{\|}\left(\partial f_{0} / \partial v_{\|}\right)+\left(n \omega_{c} / v_{\perp}\right)\left(\partial f_{0} / \partial v_{\perp}\right)}{k_{\|} v_{\|}-\left(\omega-n \omega_{c}\right)} \stackrel{\leftrightarrow}{s}\right\}, \\
& \hat{x} \\
& \hat{\mathrm{y}} \quad \hat{\mathrm{z}} \\
& \overleftrightarrow{\mathrm{S}}=\left[\begin{array}{lll}
{\left[\frac{\mathrm{n} \omega_{c}}{\mathrm{k}_{\perp}} J_{n}\right]^{2}} & i \frac{n \omega_{c}}{\mathrm{k}_{\perp}} v_{\perp} J_{n} J_{n}^{\prime} & \frac{n \omega_{c}}{k_{\perp}} v_{\|} J_{n}^{2} \\
-i \frac{n \omega_{c}}{k_{\perp}} v_{\perp} J_{n} J_{n}^{\prime} & \left(v_{\perp} J_{n}^{\prime}\right)^{2} & -i v_{\perp} v_{\|} J_{n} J_{n}^{\prime} \\
\frac{n \omega_{C}}{k_{\perp}} v_{\|} J_{n}^{2} & i v_{\perp} v_{\|} J_{n} J_{n}^{\prime} & \left(v_{\|} J_{n}\right)^{2}
\end{array}\right]
\end{aligned}
$$

The argument of the Bessel functions $J_{n}$ is $k_{1} v_{\perp} / \omega_{C} ; \omega_{C}\left(=q_{\sim} B_{0} / m\right)$ $\underset{\sim}{\mathrm{k}}=\left(\mathrm{k}_{1}, 0, \mathrm{k}_{\mathrm{z}}\right)$ and $\mathrm{B}_{0}=(0,0, \underset{\sim}{\mathrm{B}})$.

In MHD 1 imiL:

$$
\begin{aligned}
\varepsilon_{x x} & =\sum_{j} \frac{\omega_{p j}^{2}}{\omega_{c j}^{2}}\left[\left\langle\left(1-\frac{k_{\|} v_{\| j}}{\omega}\right)^{2}\right\rangle-\frac{k_{\|}^{2}\left\langle v_{1 j}^{2}\right\rangle}{2 \omega^{2}}\right] \\
\varepsilon_{x y} & =-\varepsilon_{y x}=i \sum_{j} \frac{\omega_{p j}^{2}}{\omega \omega_{c j}}\left(1-\frac{k_{\|}<v_{\| j}>}{\omega}\right)
\end{aligned}
$$




$$
\begin{aligned}
& -222- \\
& \text { A-4 } \\
& \varepsilon_{\mathrm{xz}}=\varepsilon_{\mathrm{zx}}=\sum_{j} \frac{\omega_{\mathrm{pj}}^{2}}{\omega_{\mathrm{cj}}^{2}} \frac{\mathrm{k}_{\perp}}{\mathrm{k}_{\|}}\left[\frac{\mathrm{k}_{\|}^{2}\left\langle\mathrm{v}_{\perp j}^{2}\right\rangle}{2 \omega^{2}}+\left\langle\frac{\mathrm{k}_{\|} \mathrm{v}_{\| j}}{\omega}\left(1-\frac{k_{\|} v_{\| j}}{\omega}\right)\right\rangle\right. \\
& \varepsilon_{Y Y}=\varepsilon_{x x}-\sum_{j} \frac{\omega_{p j}^{2}}{\omega_{c j}^{2}}\left(\frac{\left.k_{1}^{2}<v_{\perp j}^{2}\right\rangle}{\omega^{2}}+\frac{\left.k_{1}^{2}<v_{\perp j}^{4}\right\rangle}{4 \omega^{2}} I_{j}\right) \\
& \varepsilon_{y z}=-\varepsilon_{z y}=-i \sum_{j} \frac{\omega_{p j}^{2}}{\omega \omega_{c j}}\left(\frac{k_{1}\left\langle v_{\| j}\right\rangle}{\omega}+\frac{k_{1}}{2 k_{\|}}\left\langle v_{\perp j}^{2}>I_{j}\right)\right. \\
& \varepsilon_{z z}=-\sum_{j}\left[\frac{\omega_{p j}^{2}}{k_{\|}^{2}} I_{j}+\frac{\omega_{p j}^{2}}{\omega_{c j}^{2}}\left(\frac{\left.k_{1}^{2}<v_{1 j}^{2}\right\rangle}{2 \omega^{2}}-\frac{k_{1}^{2}<v_{\| j}^{2}>}{\omega^{2}}\right)\right]
\end{aligned}
$$

where

$$
I_{j}=\int_{-\infty}^{\infty} d v_{\|} \int_{-\infty}^{\infty} 2 \pi v_{\perp} d v_{\perp} \frac{f_{0 j}\left(v_{\perp}, v_{\|}\right)}{\left(v_{\|}-\omega / k_{\|}\right)^{2}}, \quad(\operatorname{Im} \omega>0),
$$

and

$$
\left\langle v_{j}^{n}\right\rangle=\int_{-\infty}^{\infty} d v_{\|} \int_{0}^{\infty} 2 \pi v_{1} d v_{1} v^{n} f_{0 j}\left(v_{1}, v_{\|}\right) .
$$

V. Plasma Dispersion Function

$$
\underset{\sim}{z}(\zeta)=\frac{1}{(\pi)^{1 / 2}} \int_{-\infty}^{\infty} \frac{e^{-x^{2}}}{x-\zeta} d x \text { for } \operatorname{Im} \zeta>0
$$

= analytic continuation of the above integral for $\operatorname{Im} \zeta<0$ 


$$
\begin{aligned}
& -223- \\
& \text { A-5 } \\
& z(\zeta) \simeq i(\pi)^{1 / 2} e^{-\zeta^{2}}-2 \zeta\left(1-\frac{2 \zeta^{2}}{3}\right) \text { for }|\zeta| \ll 1, \\
& z(\zeta) \tilde{=} i(\pi)^{1 / 2} \sigma e^{-\zeta^{2}}-\frac{1}{\zeta}\left(1+\frac{1}{2 \zeta^{2}}\right) \text { for }|\zeta|>>1 \text {, } \\
& \sigma=\left\{\begin{array}{l}
0 ; \operatorname{Im} \zeta>0 \\
1 ; \operatorname{Im} \zeta=0 \\
2 ; \operatorname{Im} \zeta<0
\end{array}\right.
\end{aligned}
$$


INHDMOGENEOUS

MAGNTIC FIELDS

CONTINUUM SPECTRUM (1972)

DAMPING (1949)

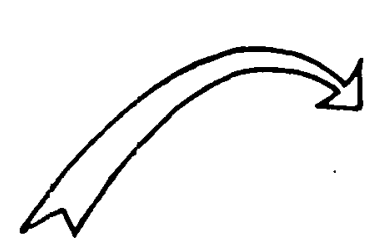

EFFECTS OF COMPRESSIBILITY ALFVÉ WAVE O TWO MAGNETOAcoustic maves (1950)

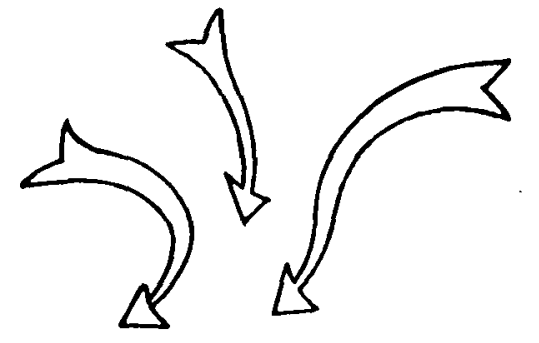

the "ideal" alfvén wave

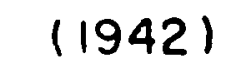

CYLINDRICAL BOUNDED SYSTEMS: ALFVÉN TORSIONAL WAVES (1949)

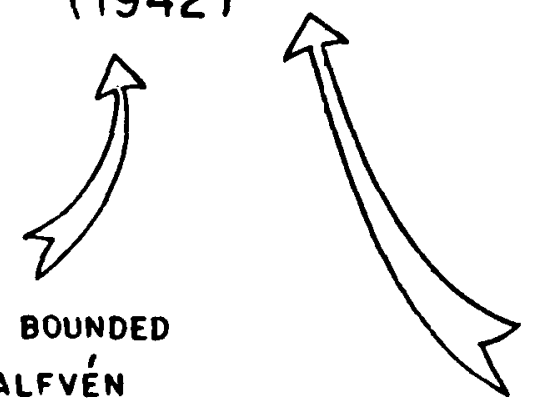

Plasmas $\omega \ll \omega_{c i}$

YIELDS THE ALFVÉN MODES

SHEAR AND COMPRESSIONAL (1950)

HALL CURRENT EFFECTS

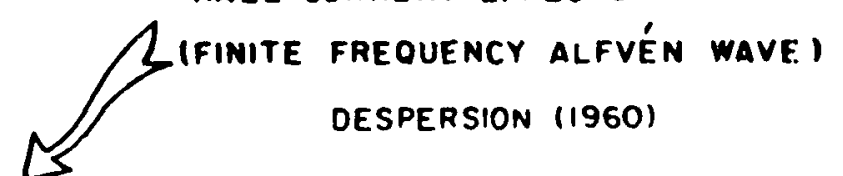

DESPERSION (1960)

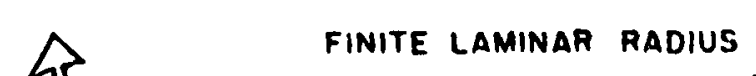

EFFECTS (KINETIC aLFVÉN WAVE)

$\stackrel{N}{N}$

THE CORIOLIS FORCE SPLITTING OF THE NORMAL MODE INTO LEFT \& RIGHT

CIRCULARLY POLARISED WAVES (1954)

\section{STUDIES OF NON-IOEALISING PARAMETERS.}




\section{FIGURE CAPTIONS}

Fig. 2.1 Measured phase velocity of Alfvén wave as a function of axial magnetic field strength, initial mass density $\rho=10^{-8} \mathrm{~g} / \mathrm{cm}$ (from

A. W. Desilva, J. M. Wilcox, W. S. Cooper and F. I. Boley; Magnetohydrodynamics, Proc. Fourth Biennial Gasdynamics Symposium, Ed. A. B. Cambel et al., North Western University Press, Evanston, 1962).

Fig. 2.2 Attenuation measured as a function of axial field strength. Solid curve is a plot of theory derived from Eq. (2.53) normalized at $12 \mathrm{~K}$ Gauss (from A. W. Desilva et al., loc. cit.).

Fig. 2.3 Oscillogram showing reflection of Alfvén wave from a pyrex end plate. The voltage reflects in phase, the magnetic field out of phase, in accord with theory for a non-conducting boundary (from A. W. Desilva et al., loc. cit.).

Fig. 2.4 Oscillogram showing reflection from a copper plate with ionizing current still flowing to the plate. The electric field has reflected out of phase and the magnetic field in phase, in agreement with theory for a conducting boundary (from A. W. Desilva et al., loc. cit.). 


\section{FIGURE CAPTIONS}

Fig. 2.5 Oscillogram showing reflection from an interface between plasma and neutral gas. The phases indicate a non-conducting boundary at reflection (from A. W. Desilva et al., 10c. cit.).

Fig. 2.6 The radial distribution of the magnetic field $b_{\theta}$, measured near the receiving end of the tube after the wave has made three transits of the tube. The dashed curve is the theoretical distribution for the lowest mode $J_{1}\left(k_{C} r\right)$. Higher modes are all neglegible at the distance represented by this measurement (from A. W. Desilva et al., loc. cit.).

Fig. 3.1 The distribution of the spectrum of $|\omega / k|$ sketched as a function of $\theta$, the angle between the wave normal direction and the direction of magnetic ficld (from C. Iherni, Phys. Fluids 15, 1673 (1972)).

Fig. 4.1 The discontinuity in desnity is shown by solid line and smooth variation by broken lines.

Fig. 4.2 The ratio of the magnetic fields, $\gamma$, and plasma densities, $n$, on either side of the plane of surface wave propagation are plotted against each other for various values of the ratio of surface wave velocity to the Alfuén wave velocity $v_{A l}$ ' $\mathrm{v}_{\mathrm{ph}} \cdot$ 


\section{FIGURE CAPTIONS}

Fig. 4.3 The branch cuts and the least-damped roots of the dispersion function $D(k, \omega)$ on the $n=-1$ sheet of its Riemannian surface.

Fig. 4.4 Schematic diagram of $E_{x}$ field of the kinetic Alfvén wave excited at the Alfvén resonance surface $x=0 . \quad E_{0}$ shows the $x$ component of the external field or the surface Alfvén wave. The Alfvén speed is assumed to decrease toward the right.

Fig. 6.1 Dispersion diagram to show the decay process of the kinetic Alfvén wave at $\omega_{0}$ to another kinetic Alfvén wave at $\omega_{A}$ and the ion acoustic wave at $\omega_{s} \cdot$

Fig. 6.2 Nonlinear potential function $F$ versus the normalized number density. The solitary wave solution exists when $F \geq 0$.

Fig. 6.3 The normalized density as a function of $\mathrm{n} / \mathrm{K}_{\mathrm{x}}$.

Fig. 6.4 The normalized electric $\overline{\mathrm{E}}_{\mathrm{x}}$ and magnetic $\overline{\mathrm{B}}_{\mathrm{y}}$ field plotted as a function of $n / K_{x}$.

Fig. 6.5 The magnetic line of force of the kinetic Alfvén solitary wave has a kink structure as shown by the curve with arrows.

Fig. 7.1 Variation of $\mathrm{E}^{\circ}$ as defined in Eq. (7.8) and the location of the surface current $J_{s}$ to excite the kinetic Alfvén wave for plasma heating. 


\section{FIGURE CAPTIONS}

Fig. 7.2 Alfvên wave heating experiments on thẹ Lausannelinear theta pinch (Keller and Pochelon, 1978).

Fig. 7.3 Plasma temperature and $\beta$ value in the presence (solid circle) and the absence (open circle) of the Alfvên wave (Keller and Pochelon, 1978).

Fig. 7.4 Experimental setup of the Wisconsin proto cleo (Golovato et al., 1976).

Fig. 7.5 Change of temperature as a function of radial position in the proto cleo Alfvern wave heating (Golovato et al., 1976).

Fig. 7.6 Experimental setup of Suhumi RO-2 Stellerator (Demirhanov et al., 1977).

Fig. 7.7 Diamagnetism which indicates the plasma temperature as a function of Alfvén wave intensity. A clear nonlinear heating is seen. (Demirhanov' et al., 1.977).

Fig. 7.8 Experimental setup of Kyoto Stellerator (Obiki et al., 1977).

Fig. 7.9 Electron temperature profile with and without Alfvén wave as a function of radial position and time (Obiki et al., 1977).

Fig. 7.10 Heating threshold as a function of the initial electron temperature in the Kyoto heliotron (Obiki et al., 1977). 


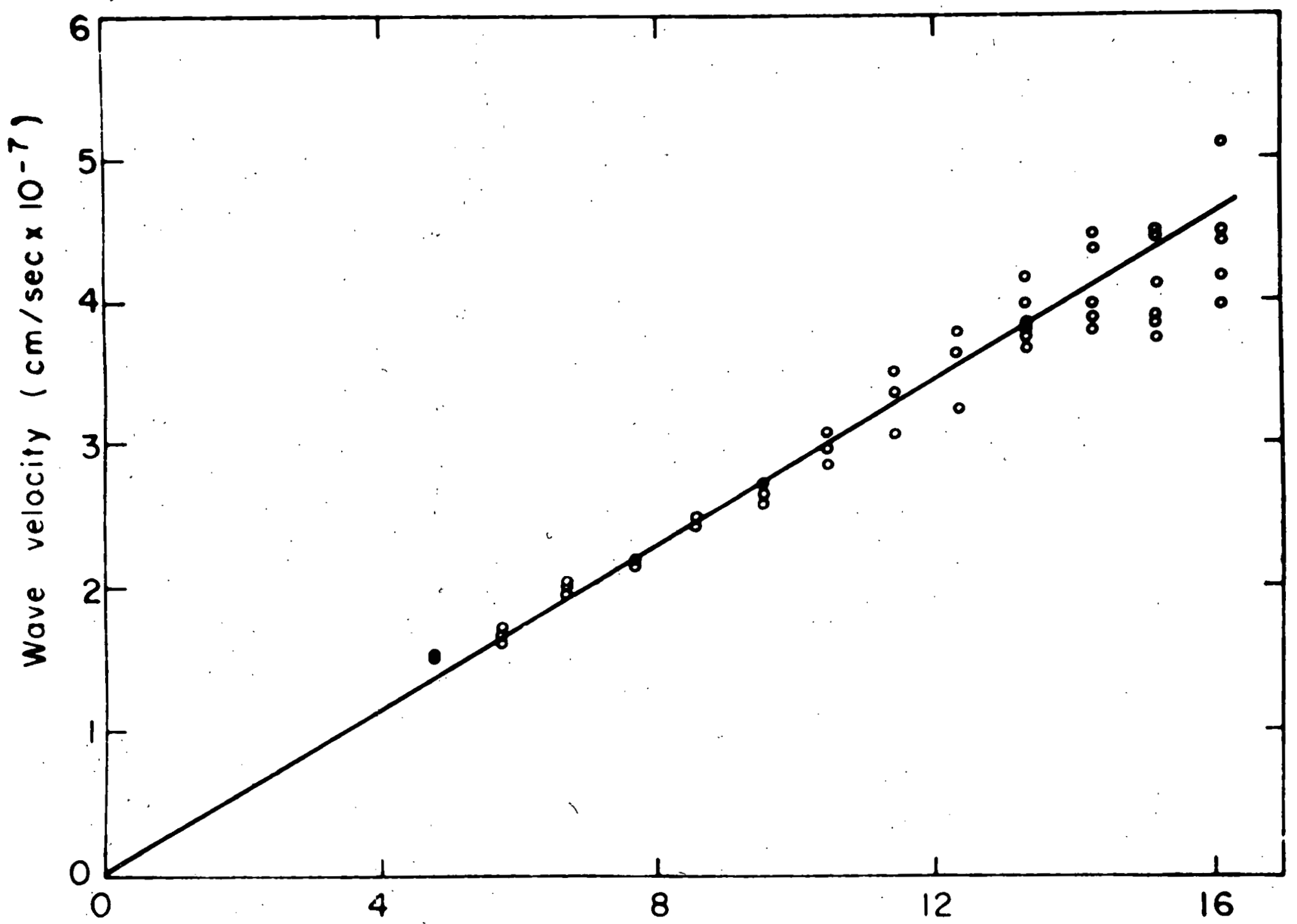

Axial magnetic field ( $k$ gauss) 


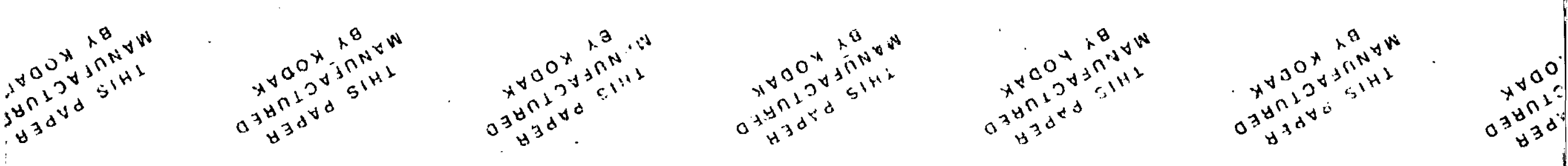

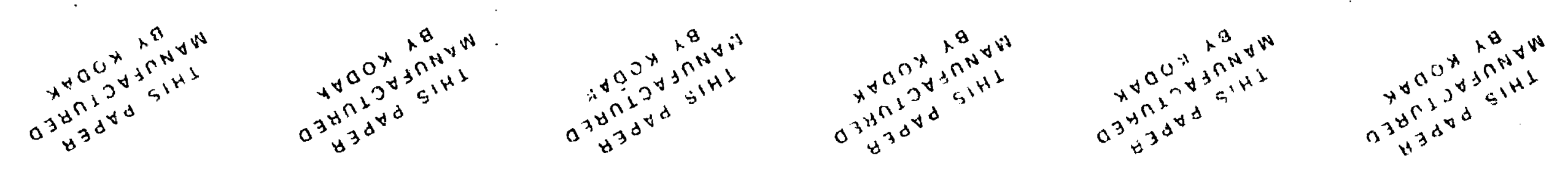

然

$$
\text { 赵 }
$$

等

"




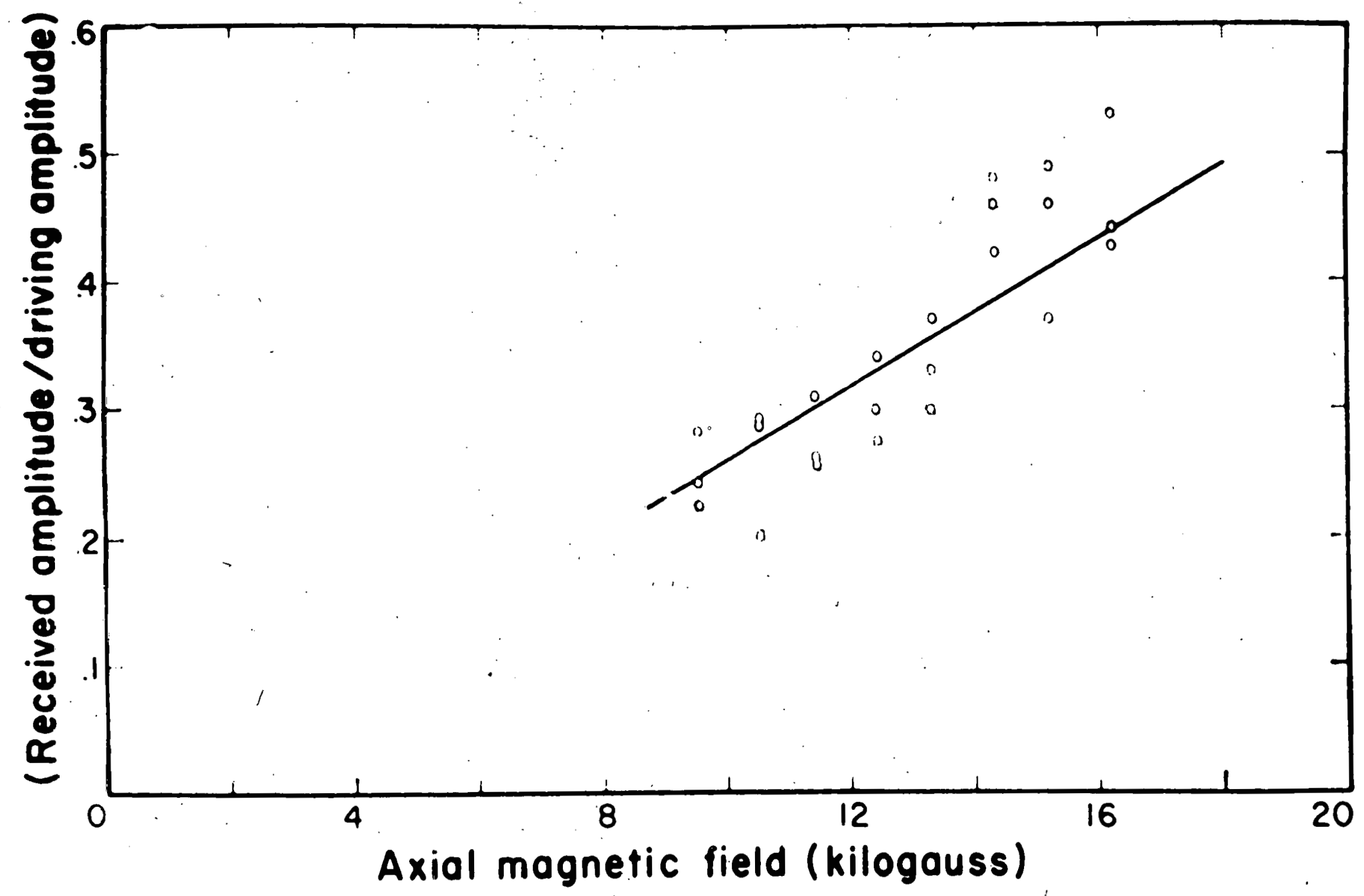




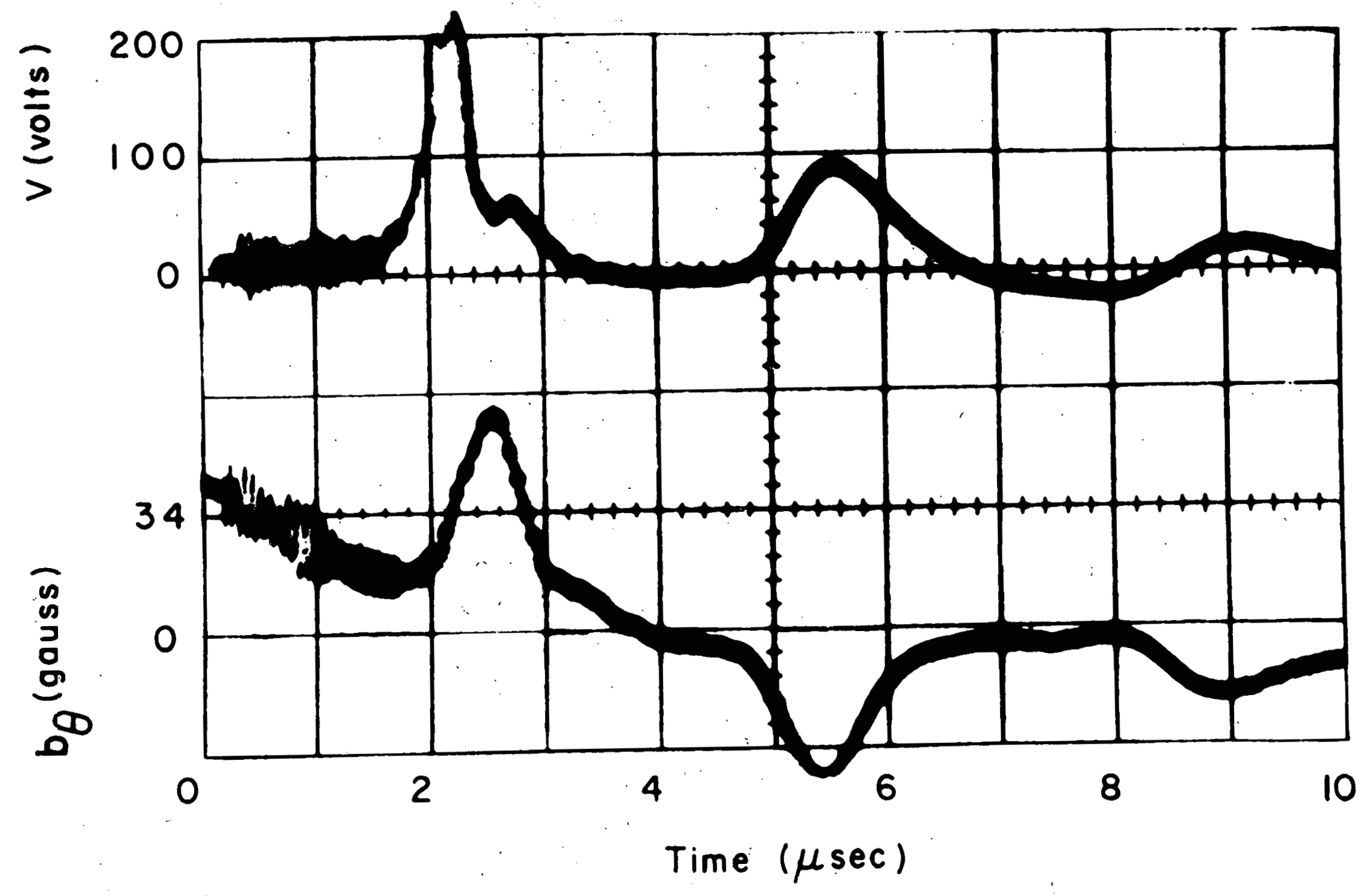




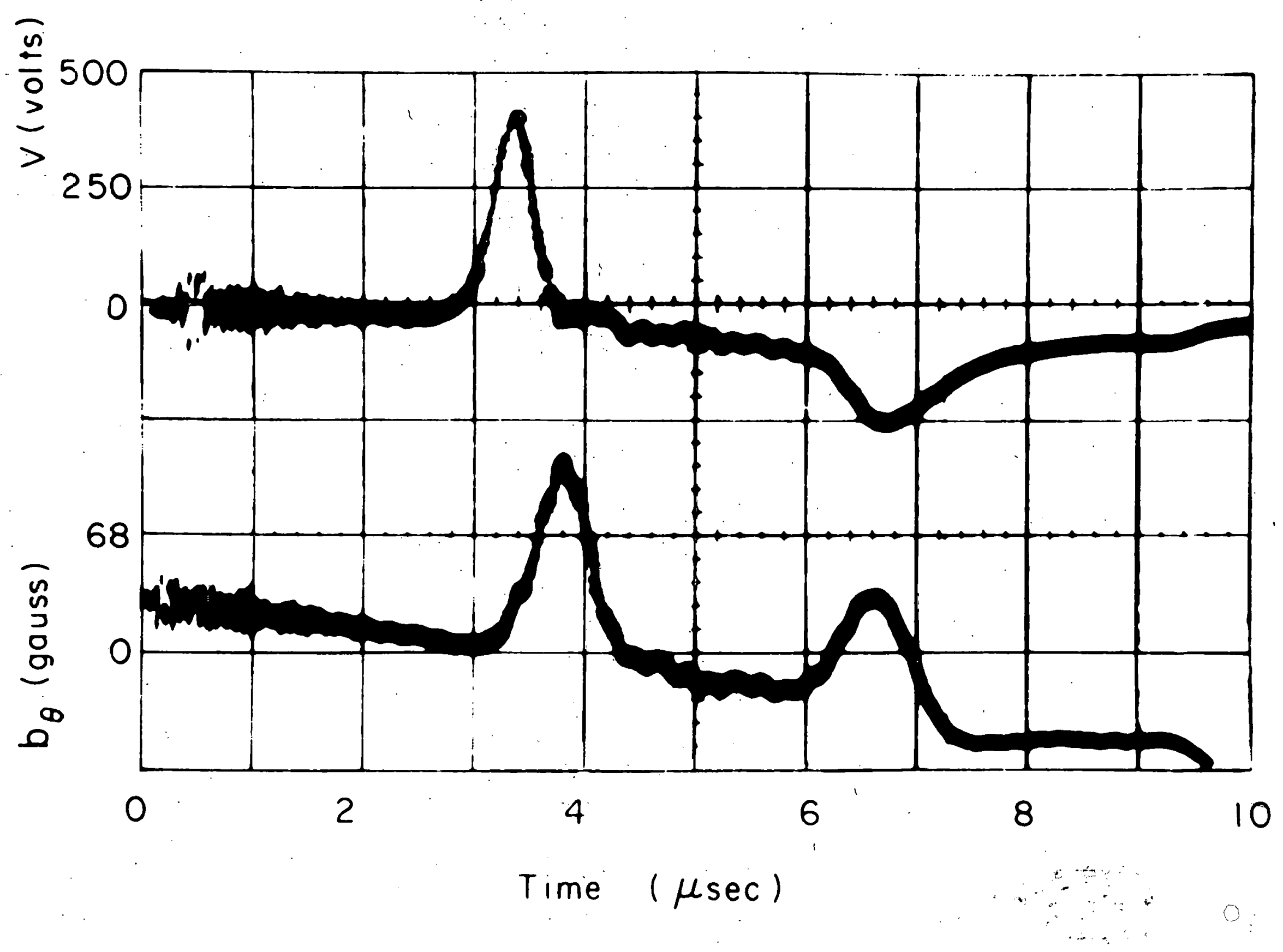


Fig. $2.4 \mathrm{H}-\mathrm{U}$ 


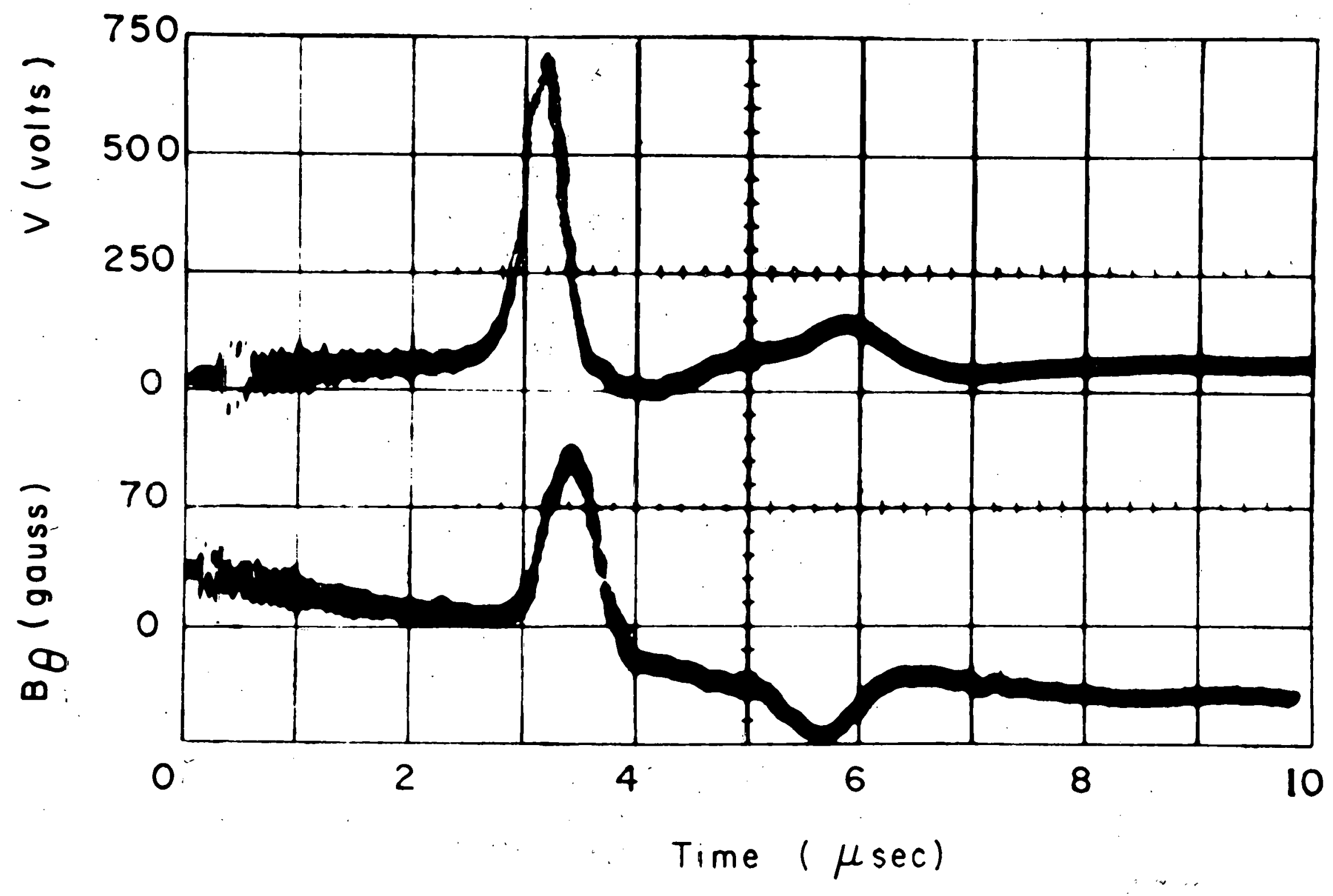


Fig. $2.5 \mathrm{H}-\mathrm{U}$ 


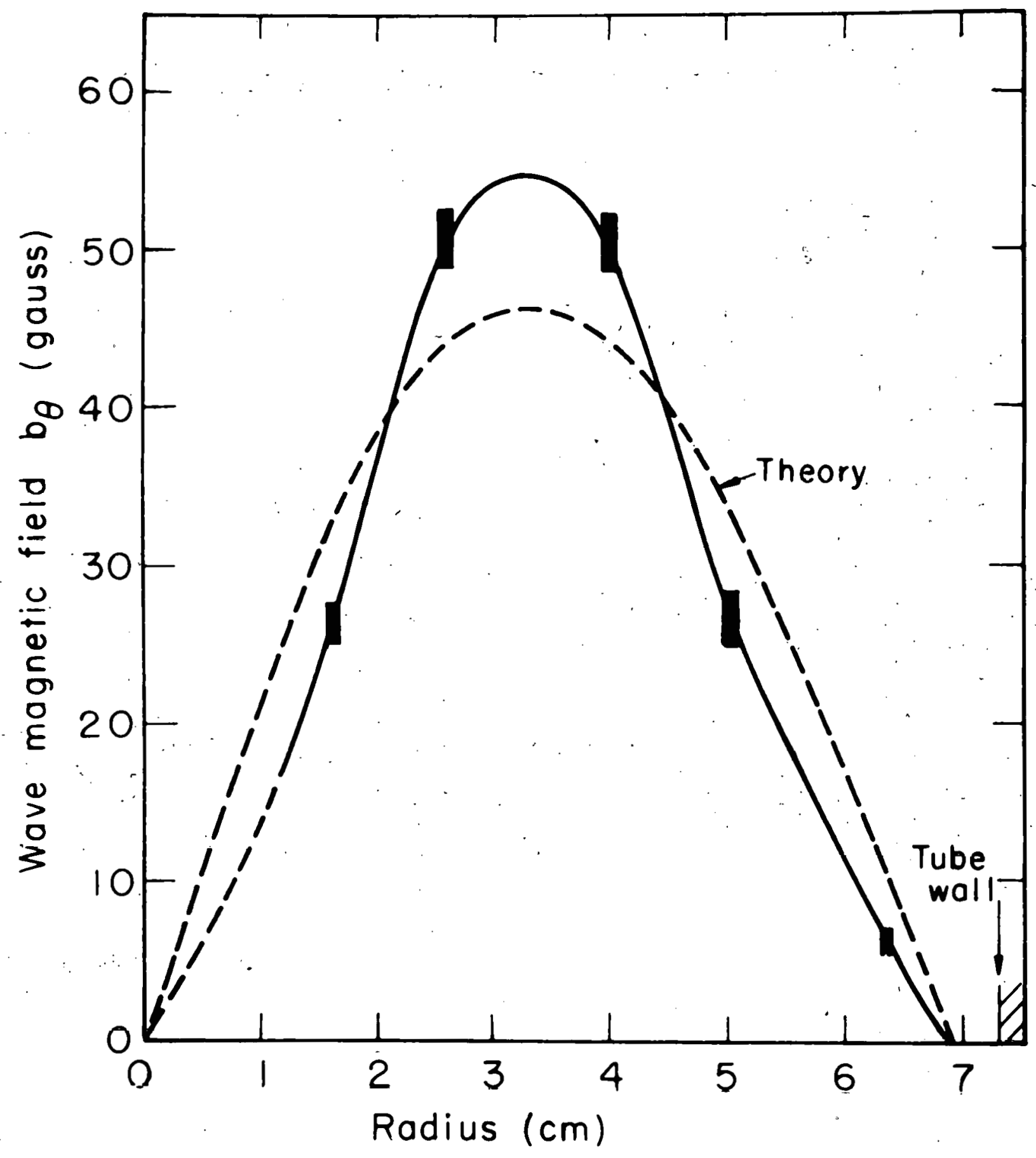




$$
\begin{aligned}
& 0_{0}^{0,100}
\end{aligned}
$$

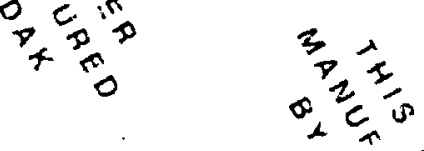

$$
\begin{aligned}
& 00
\end{aligned}
$$

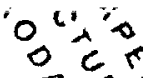

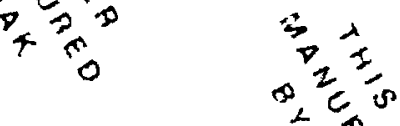

$$
\begin{aligned}
& 7 p_{0}^{2} \quad 3 \\
& x^{2} 0 \% \\
& 32 \\
& \text { 年 } \\
& 0.2= \\
& 100 \\
& +0,00 \\
& \text { + }
\end{aligned}
$$

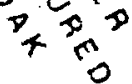

$$
\begin{aligned}
& \text { 年事 } \\
& \text { 华 } \\
& 0
\end{aligned}
$$

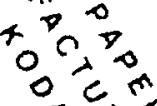

$$
\begin{aligned}
& \text { 年 } \\
& 0 \frac{2}{2} \frac{1}{4} \\
& 30 \frac{3}{2} \\
& \text { r } \\
& 0, e^{2} \\
& \text { 4 } 70
\end{aligned}
$$

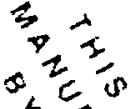

$$
\begin{aligned}
& p
\end{aligned}
$$

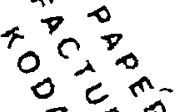

$$
\begin{aligned}
& \text { T D } \\
& 0, \frac{3}{2} \frac{1}{5} \\
& +70.0
\end{aligned}
$$

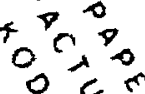

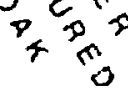

$$
\begin{aligned}
& \int_{0}^{0} \\
& \text { o) } \frac{7}{2}= \\
& +0
\end{aligned}
$$

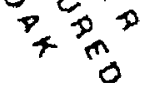

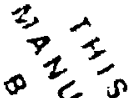

$$
\begin{aligned}
& 3=\frac{3}{5} \\
& \text { 焉然 } \\
& \text { 年 } \\
& 0 \text { e } \\
& 02 \frac{1}{2} \\
& \text { तs }
\end{aligned}
$$

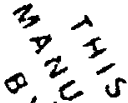

$$
\begin{aligned}
& \text { स० } 0
\end{aligned}
$$

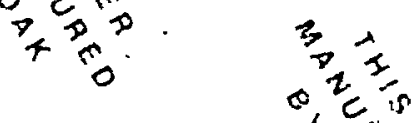

$$
\begin{aligned}
& \text { 每 } \\
& \text { 落 } \\
& 3 \frac{3}{2} \frac{1}{2}
\end{aligned}
$$

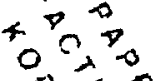

$$
\begin{aligned}
& \text { 每 } \\
& 0 \frac{7}{2} \frac{1}{2} \\
& 40 \\
& \text { 每 } \\
& =\frac{1}{20}
\end{aligned}
$$

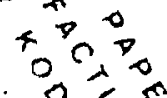

$$
\begin{aligned}
& 7 \times \\
& +0 \\
& 0, \frac{2}{2}=0
\end{aligned}
$$

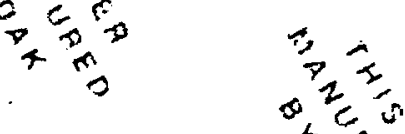

$$
\begin{aligned}
& 100 \% \\
& \text { 然 }
\end{aligned}
$$

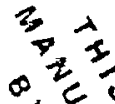

$$
\begin{aligned}
& \text { 年 } \\
& \text { F } \\
& \text { * } \\
& 02 \frac{3}{2} \\
& \text {, } \\
& 40
\end{aligned}
$$

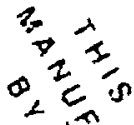

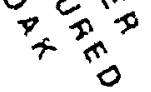

$$
\begin{aligned}
& \left.0, \frac{2}{2}\right)^{2}-\frac{1}{5} \\
& \text { 等 } \\
& \text { 急 }
\end{aligned}
$$

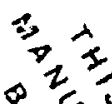

$$
\begin{aligned}
& 0_{2} \frac{z}{2} \\
& 58 \\
& \text {, } \\
& \text { से }
\end{aligned}
$$

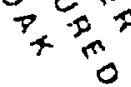

$$
\begin{aligned}
& \text { से } \\
& 3 \\
& \text { \% } \\
& \text { of } \\
& \text { स० } \\
& \text { 策 } \\
& \text { is } \\
& +00 \\
& \text { 年 } \\
& \text { rok } \\
& 02 \frac{2}{5} \\
& \text { से० } \\
& \text { 争皮 } \\
& \sum_{2} e_{0}=0 \\
& 0 \frac{2}{2} \frac{5}{5} \\
& \text { से } \\
& \text { P }
\end{aligned}
$$

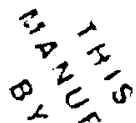

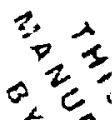

$$
\begin{aligned}
& \text { 要 }
\end{aligned}
$$

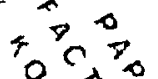

$$
\begin{aligned}
& \text { + } \\
& 0 \\
& 3 \\
& 0 \\
& \text { + } 00
\end{aligned}
$$




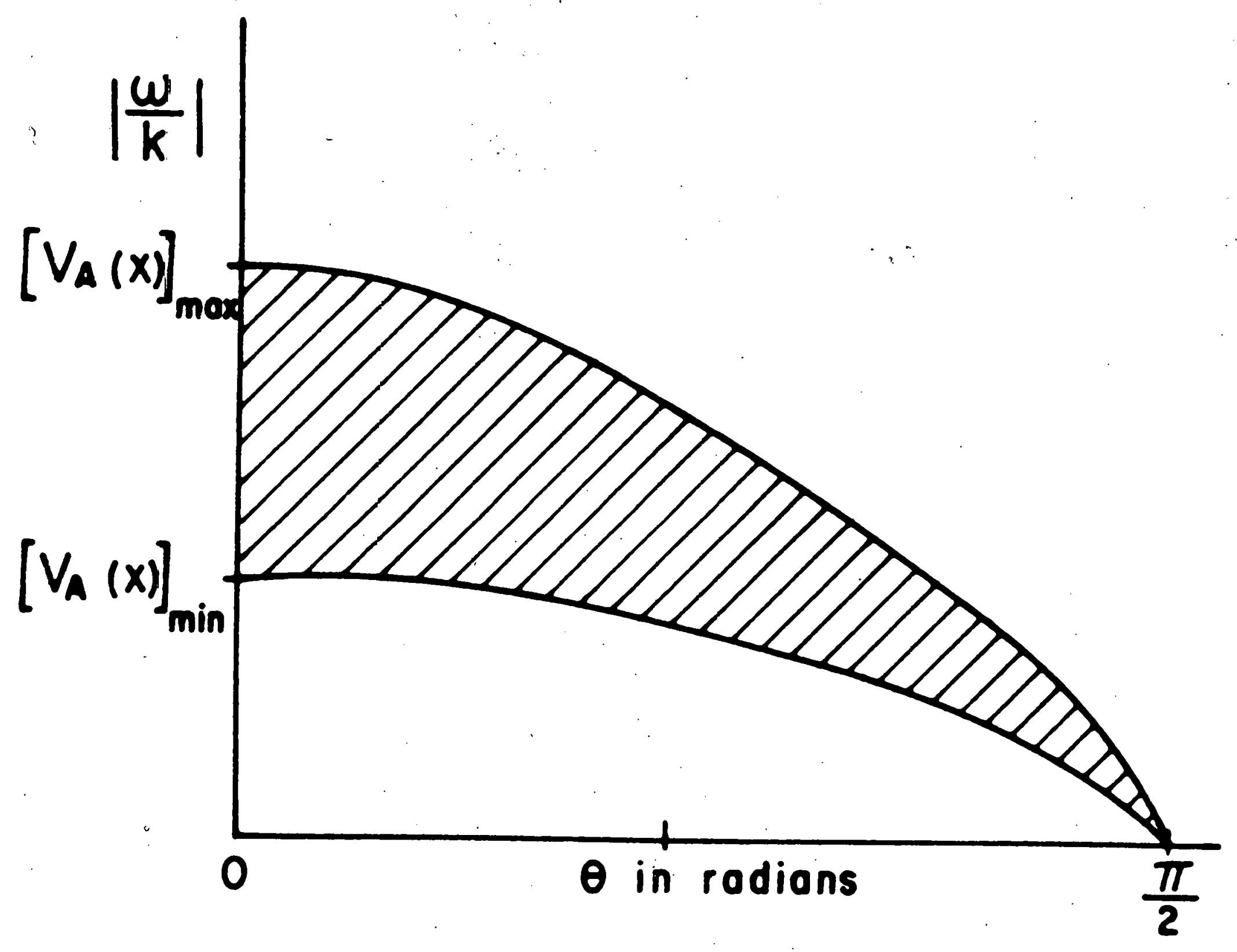




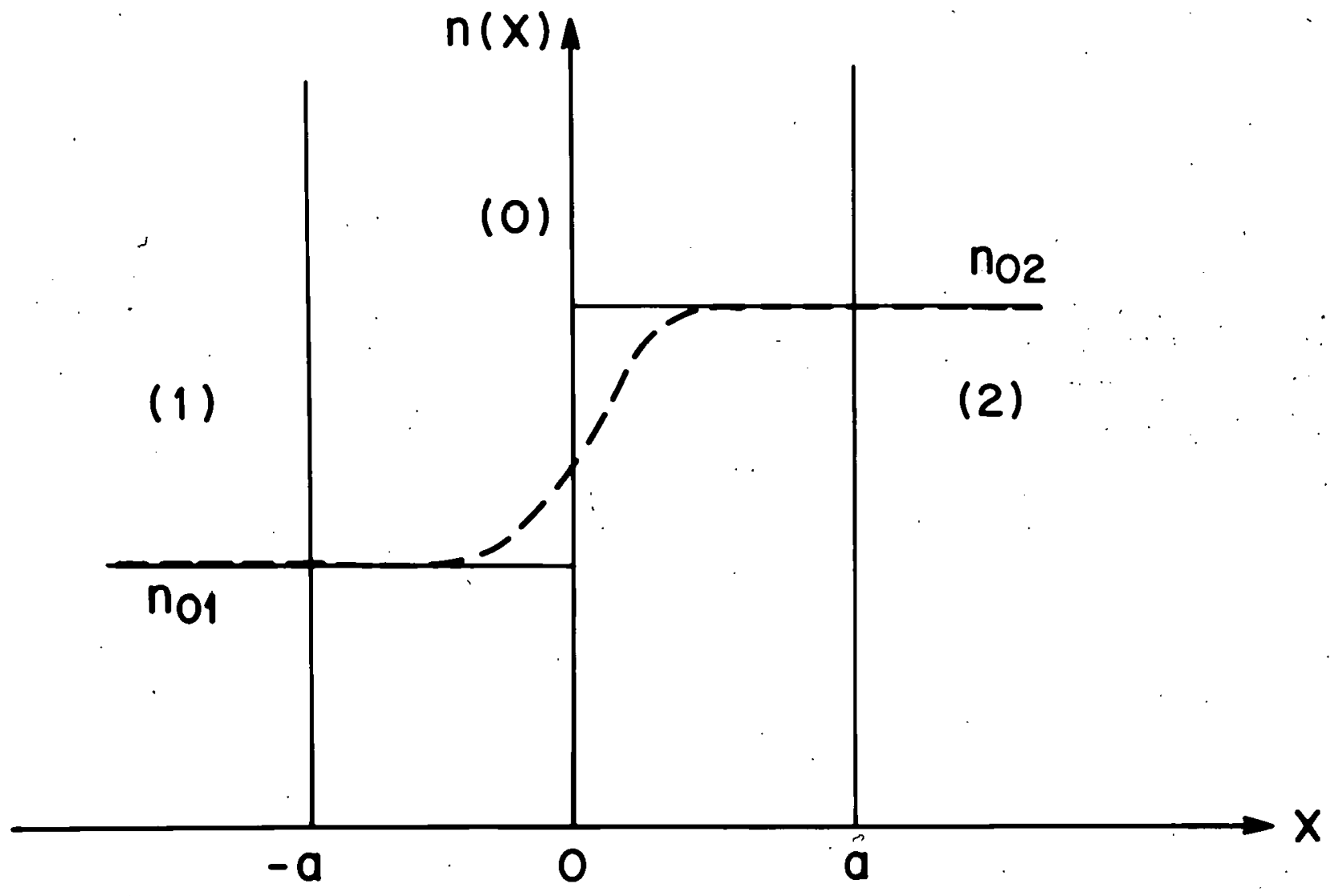


$[F i g .4 .1, H-j]$ 


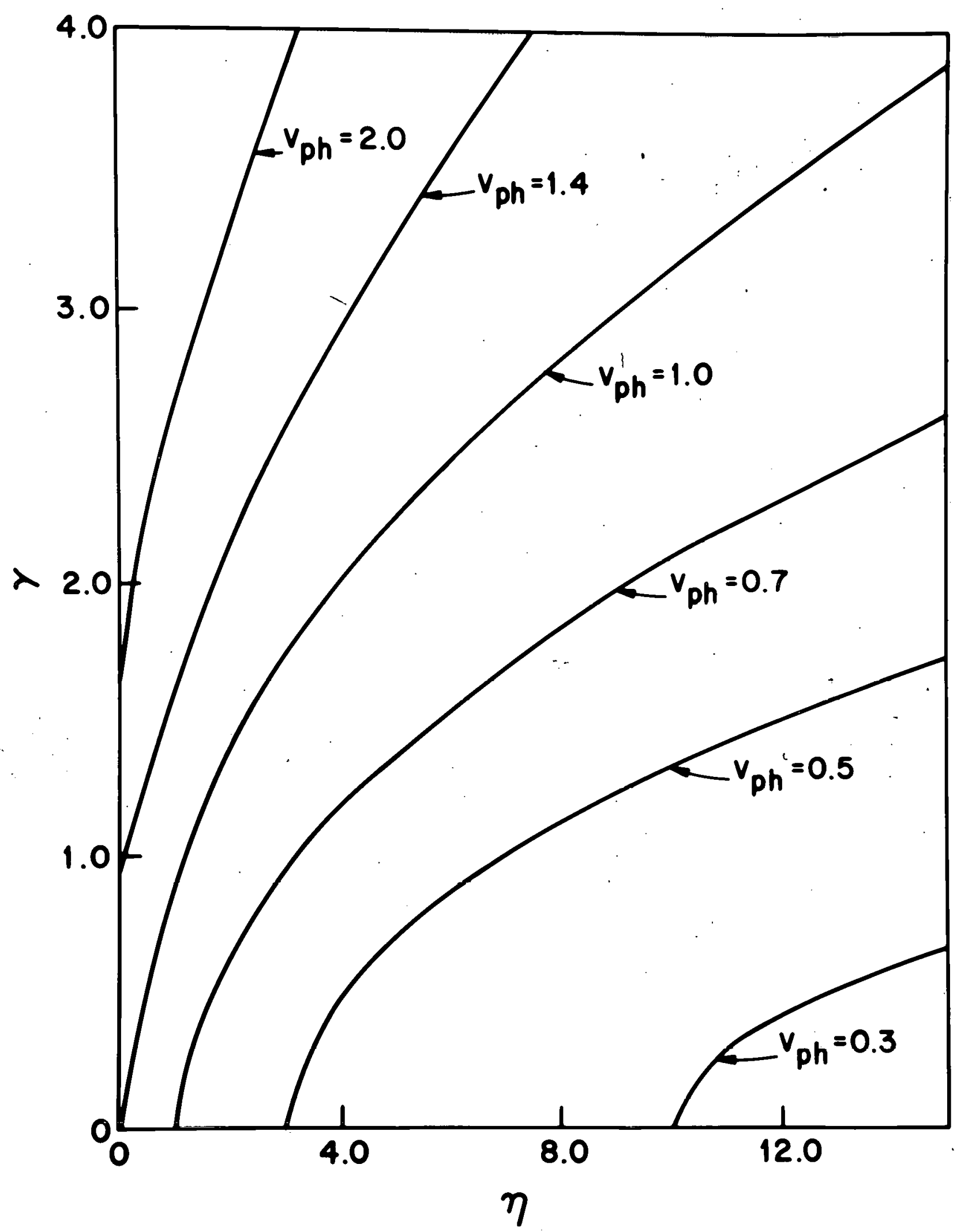


Fig $4,2+10 \div 0 \div$ 


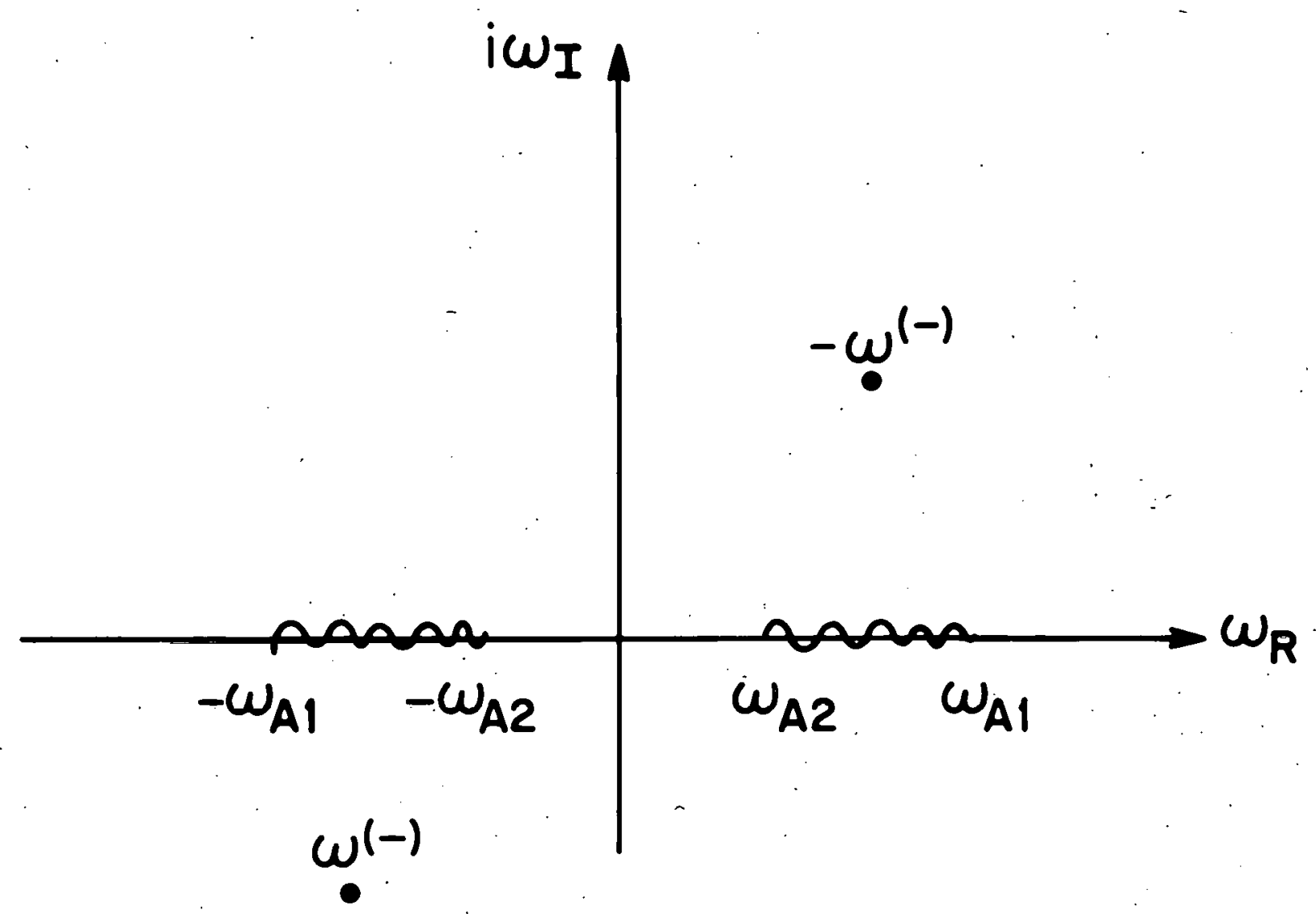


$F i g, 3 H-U$ 


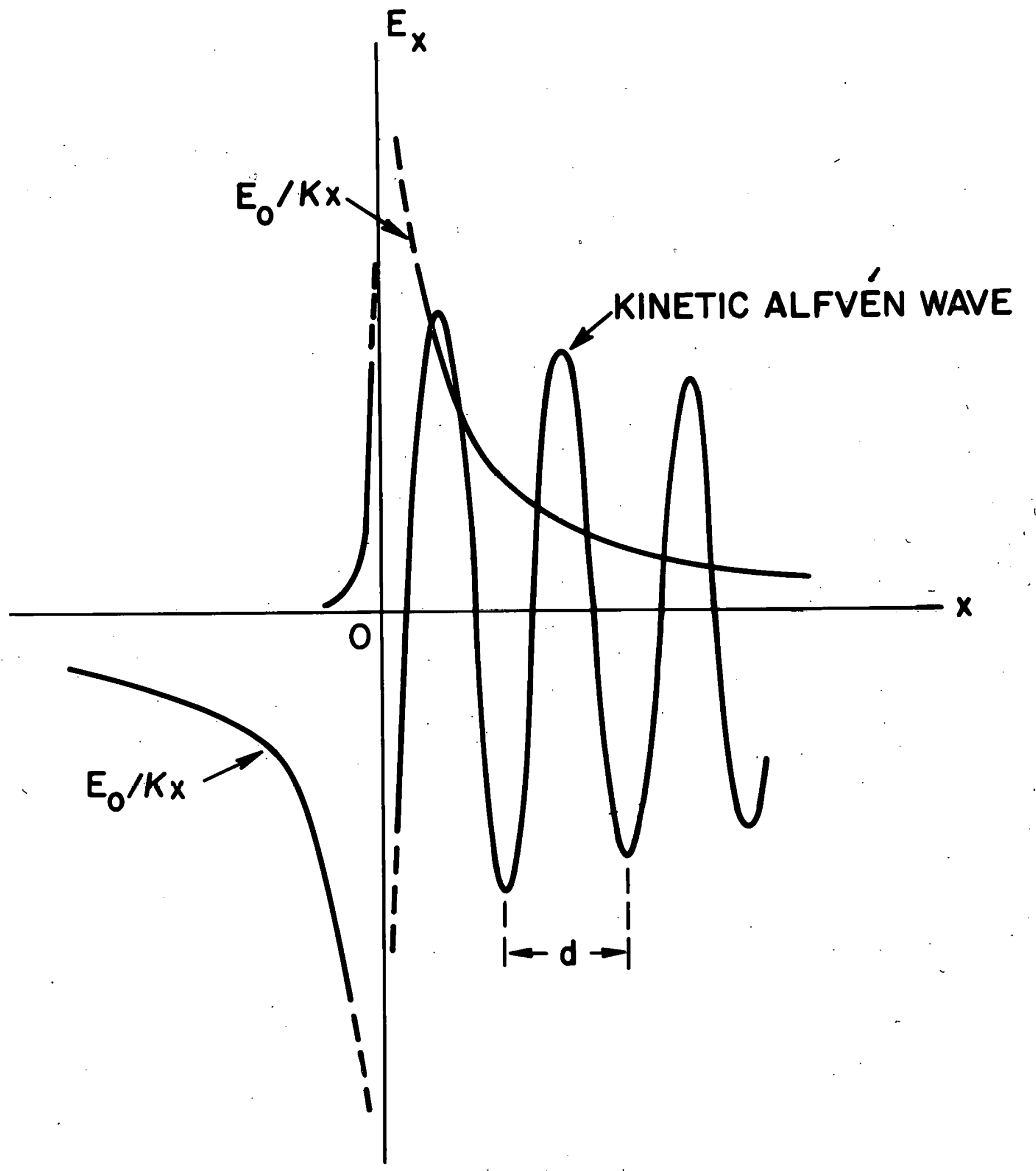





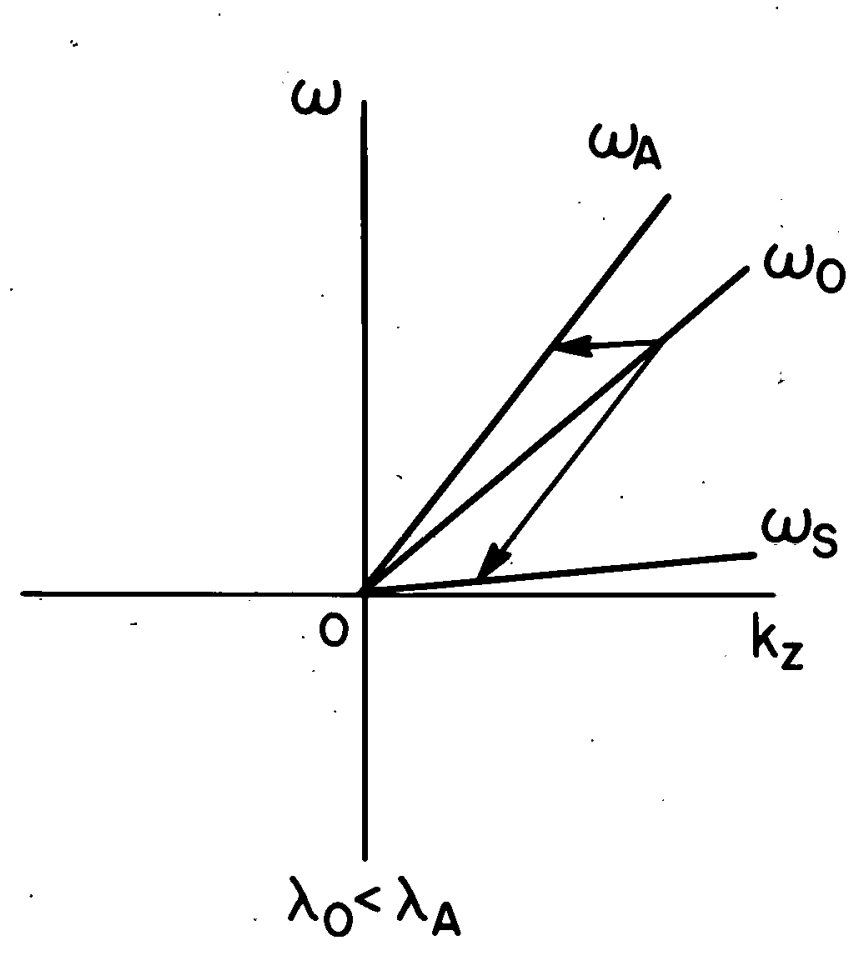

(a)

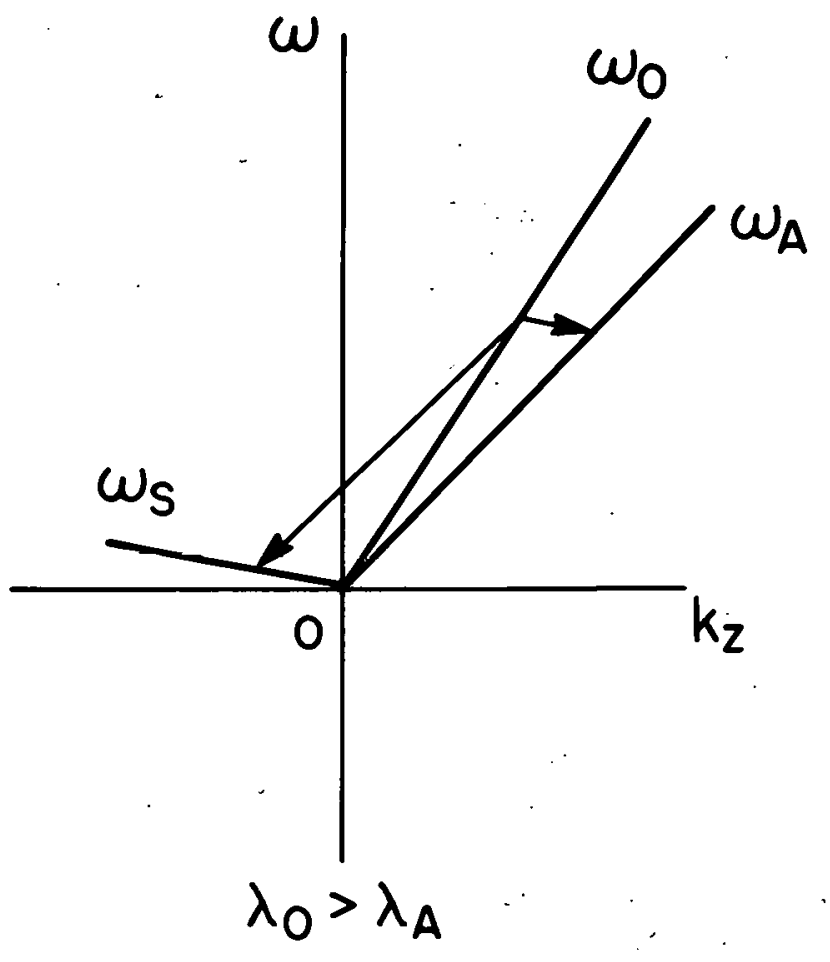

(b)

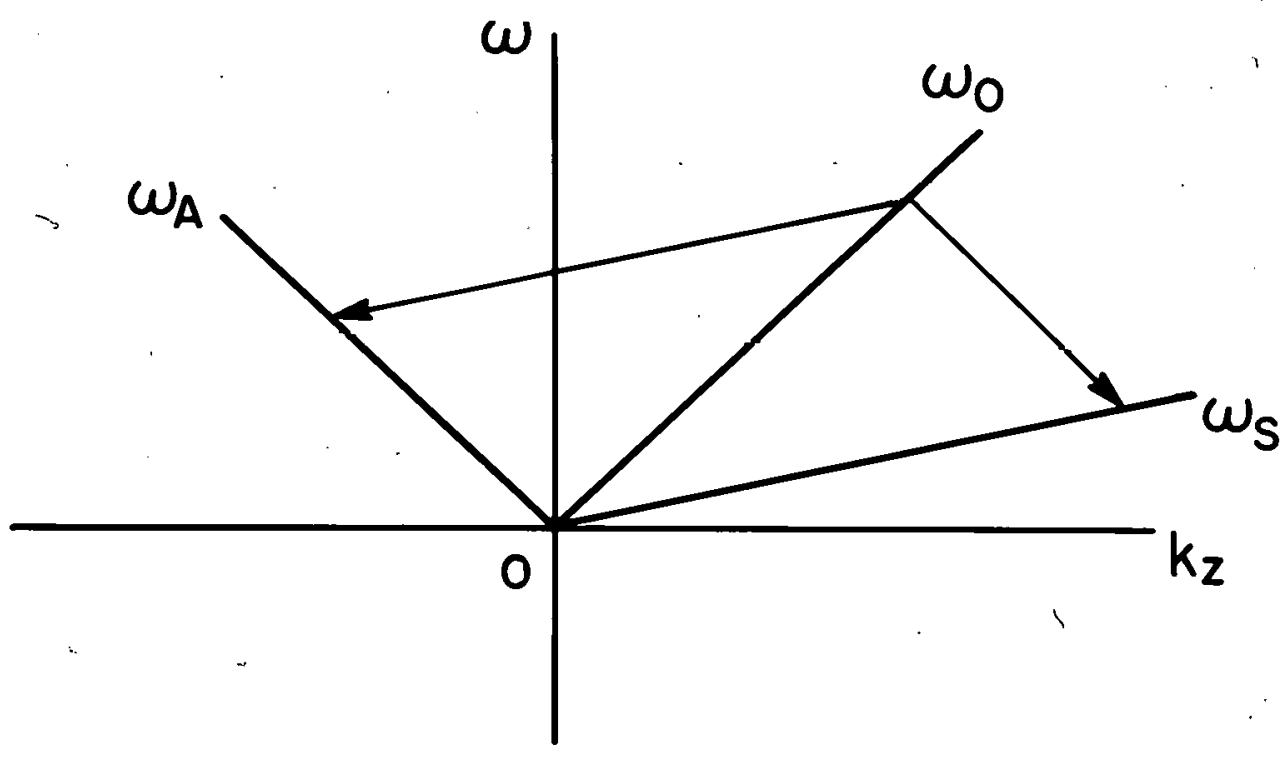

$$
\lambda_{0} \gtrless \lambda_{A}
$$

(c) 


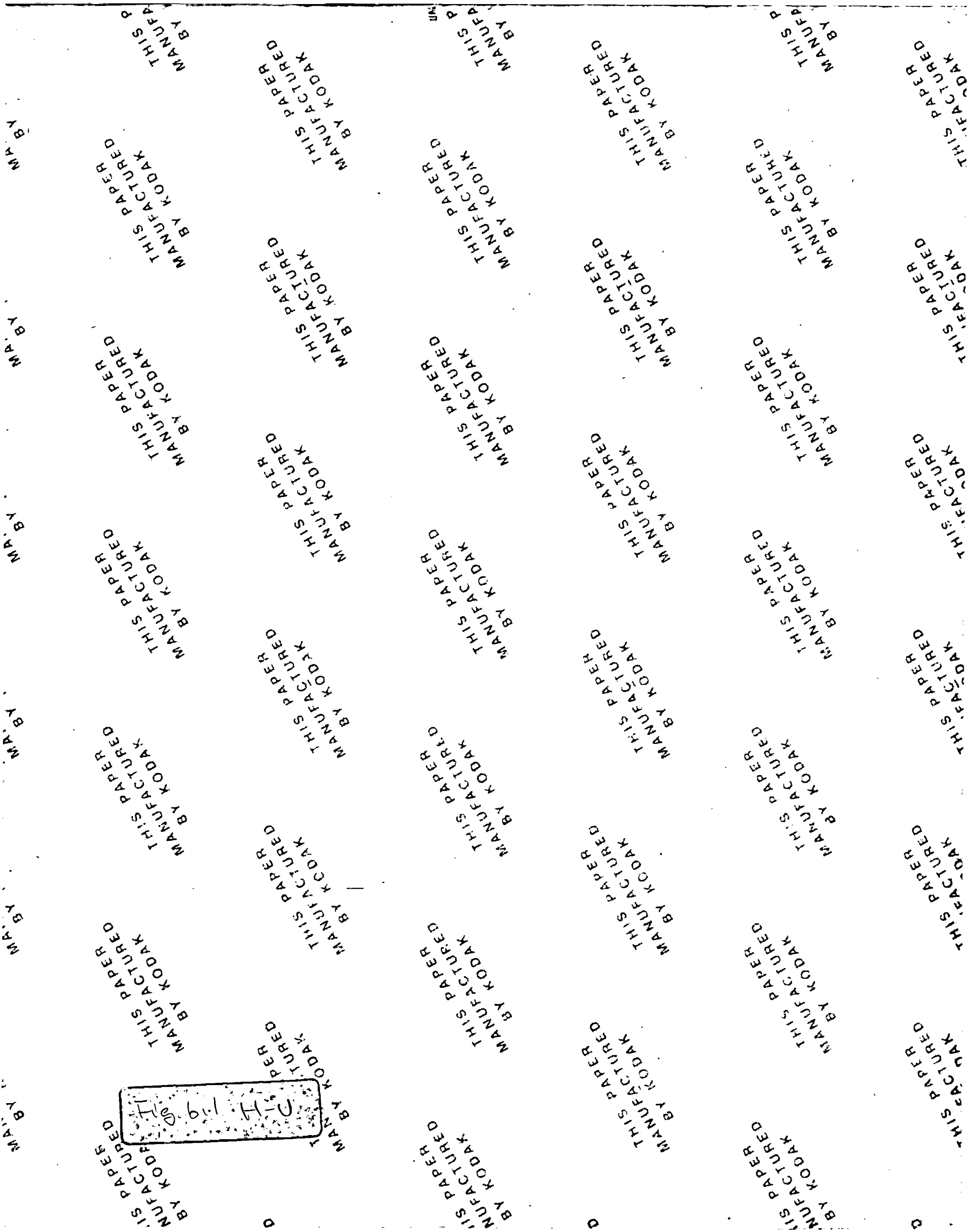




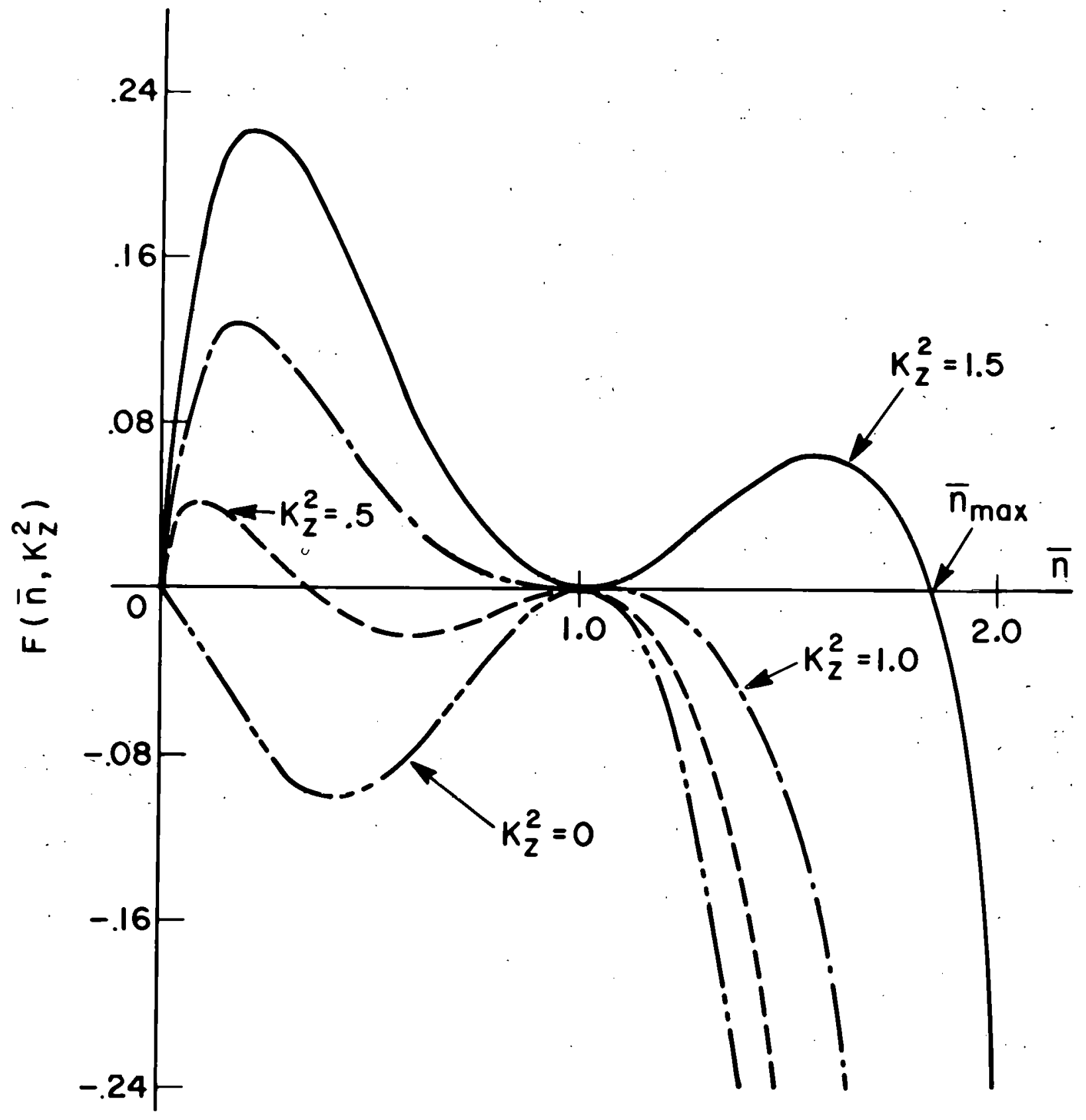




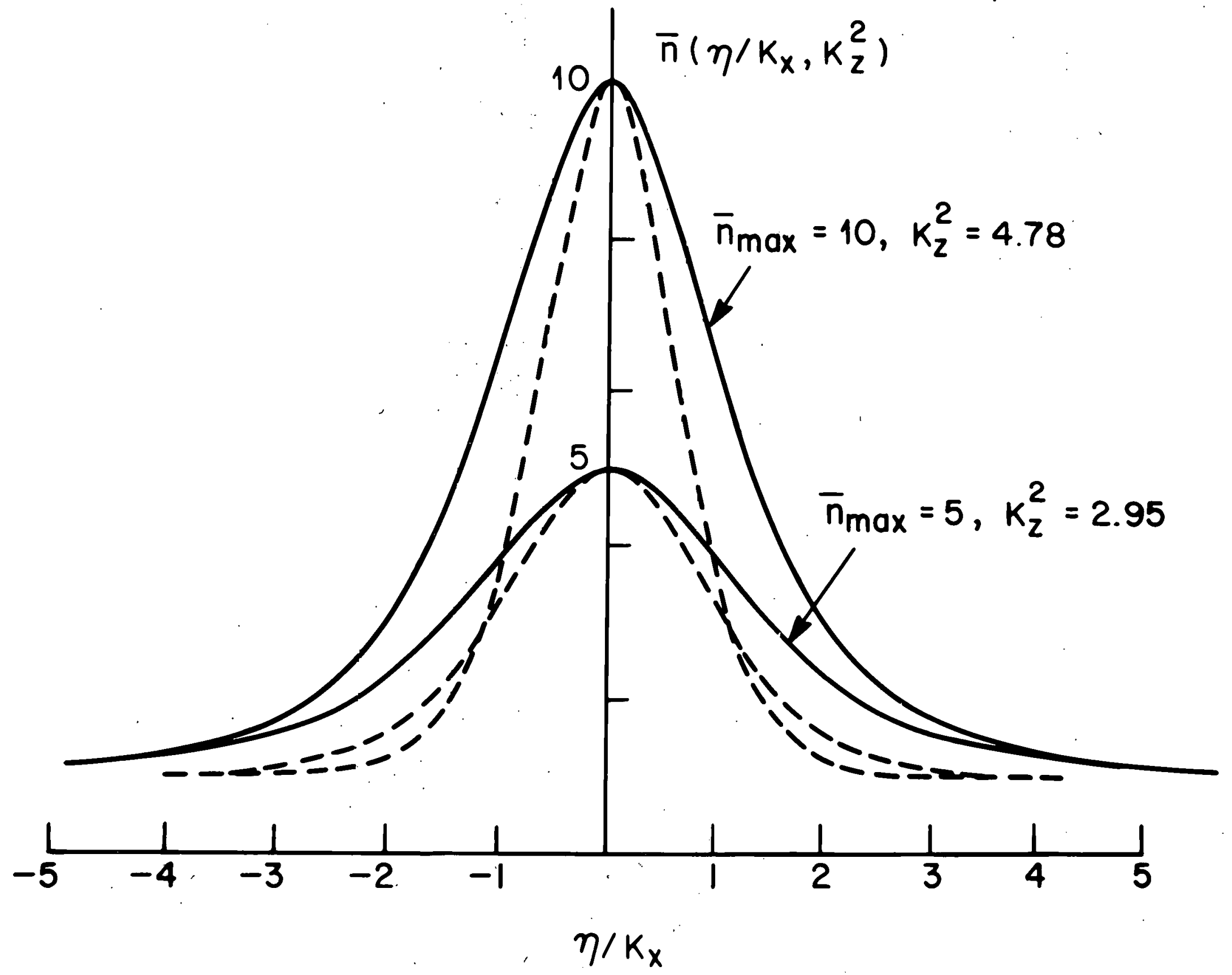




$$
\text { Fig. } 6.3 \mathrm{H}-\mathrm{U}
$$




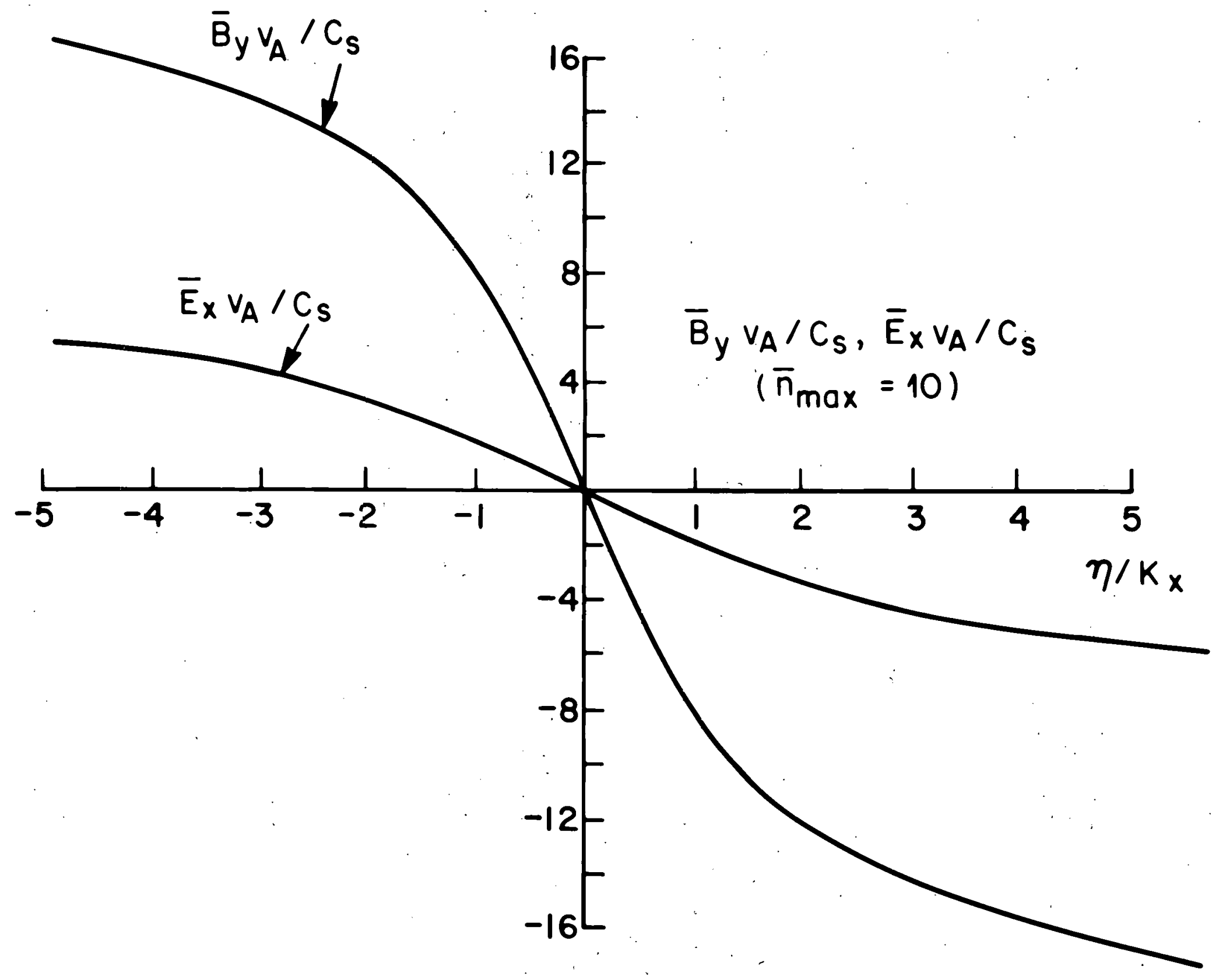


$\operatorname{Fig} 6,4 \mathrm{H}-\mathrm{J}$ 


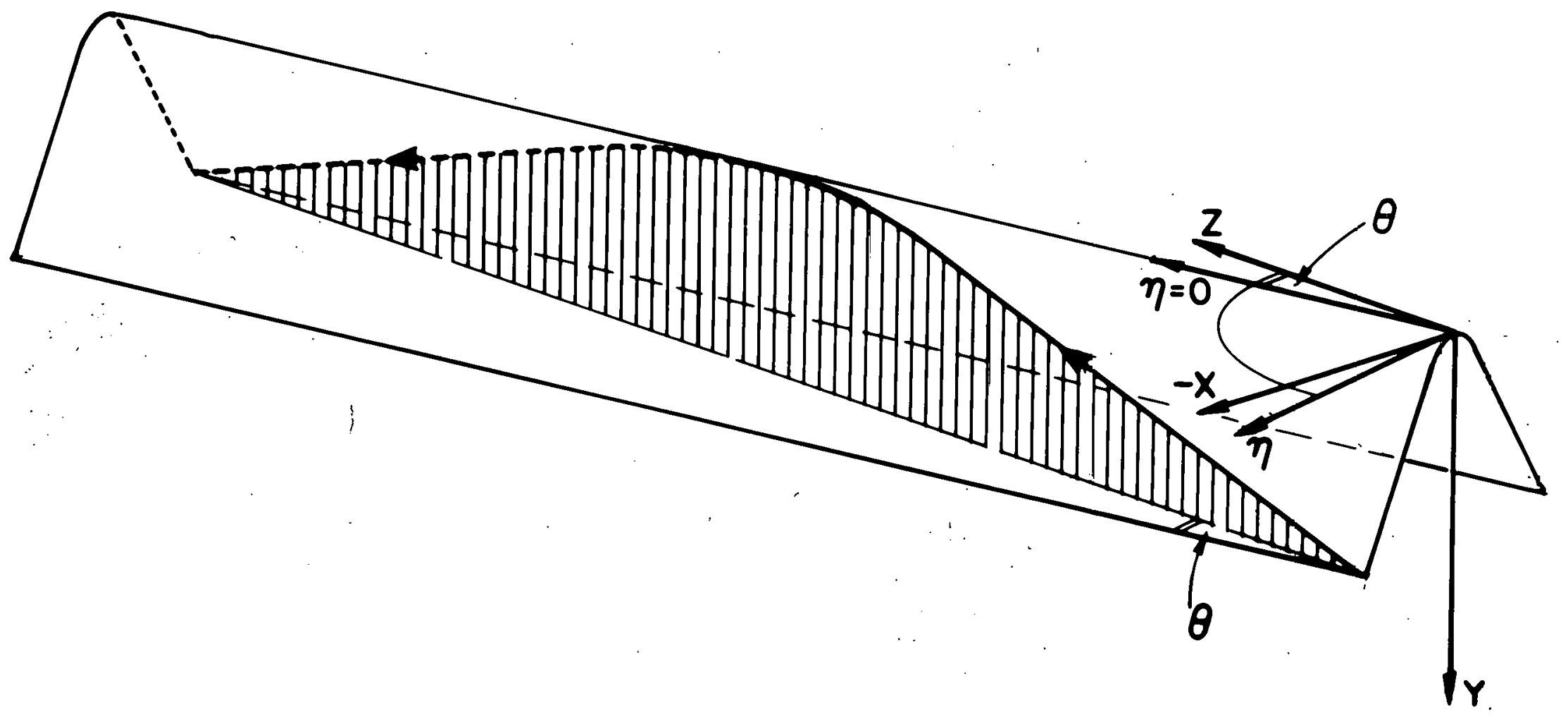




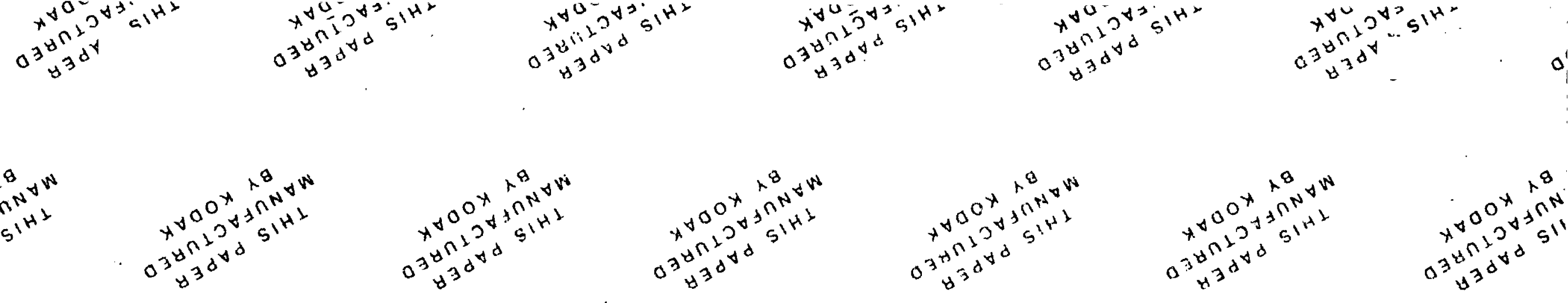

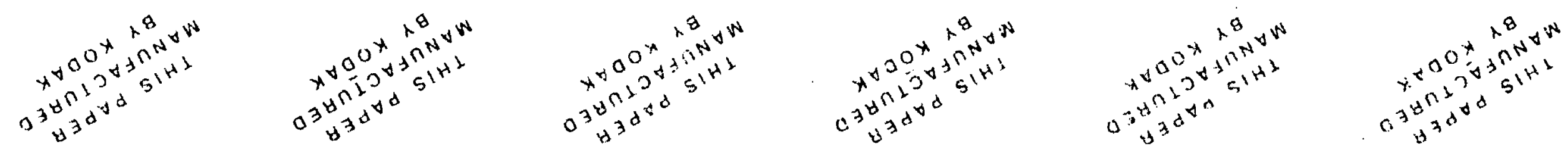

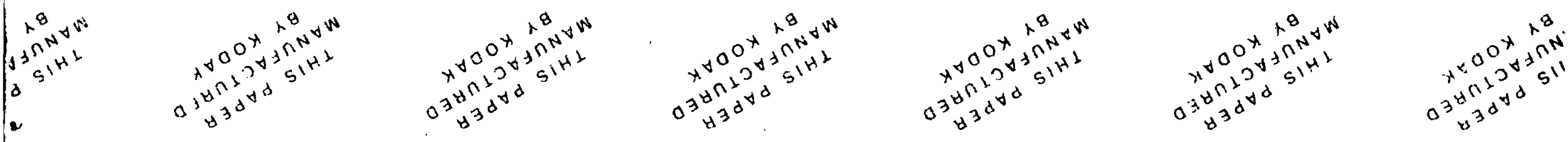

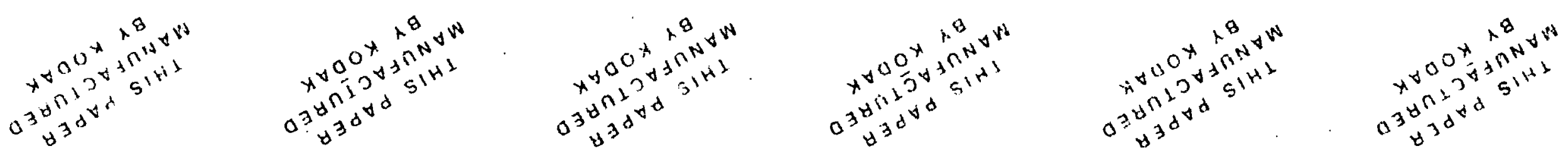

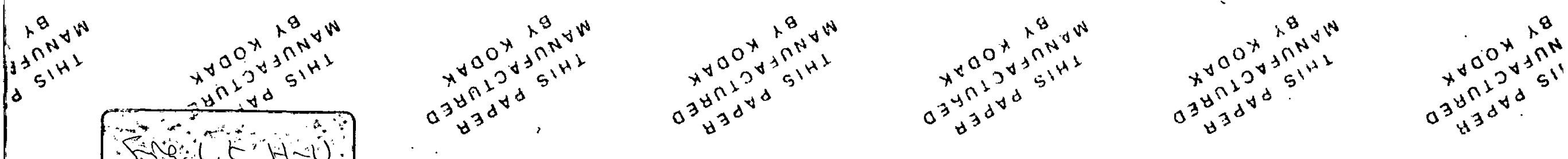
$-x^{8}+4$
$\therefore x \sin ^{n} d x$
$n+5 n_{0}$
$n+\operatorname{sen}_{0}$
$n+r^{3}+N$
$.0 \times . a_{0}^{n}+4$ 


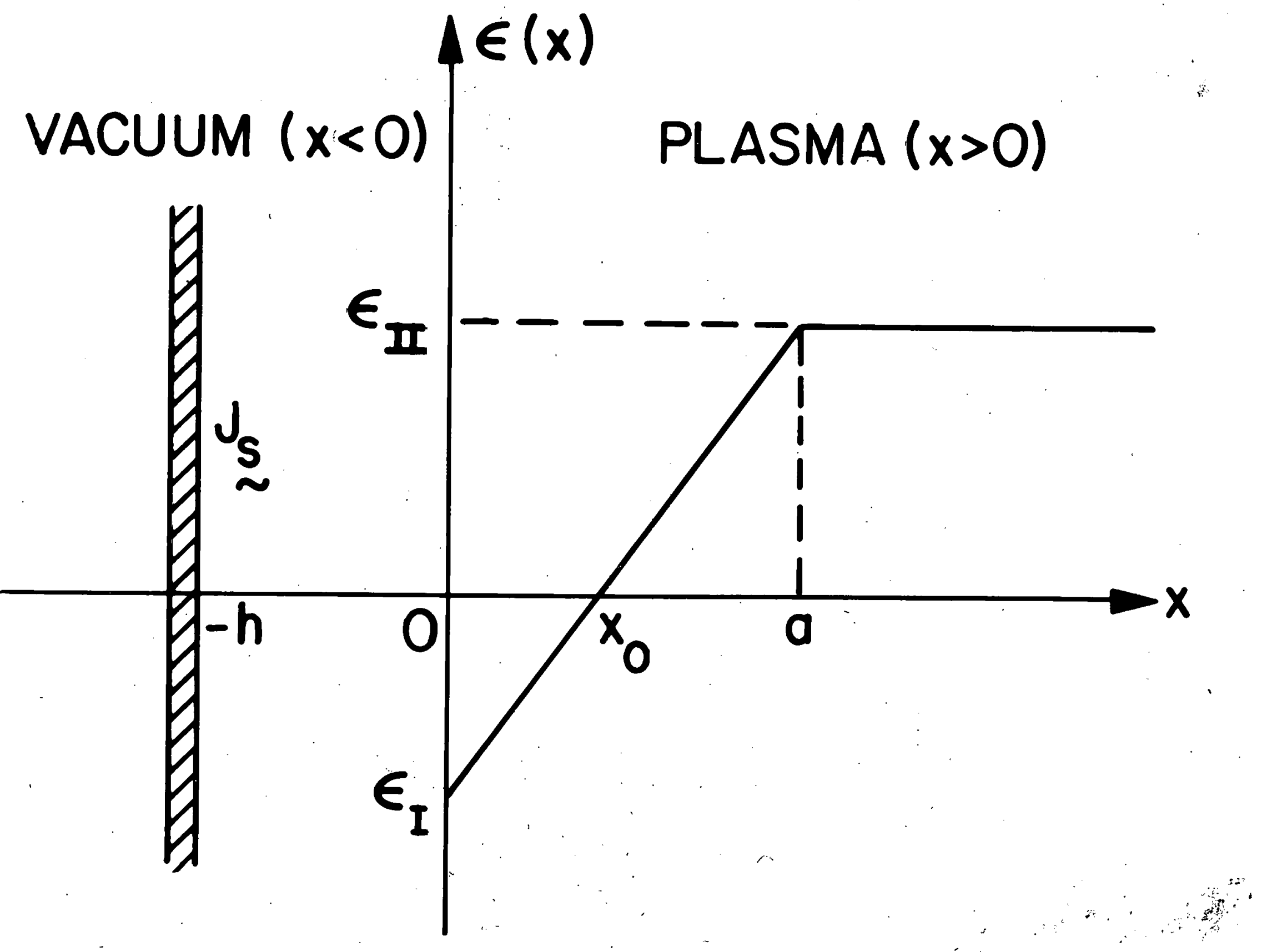


Fig. $7.1 \mathrm{H}-\mathrm{U}$ 


\section{LAUSANNE $\theta$ PINCH}

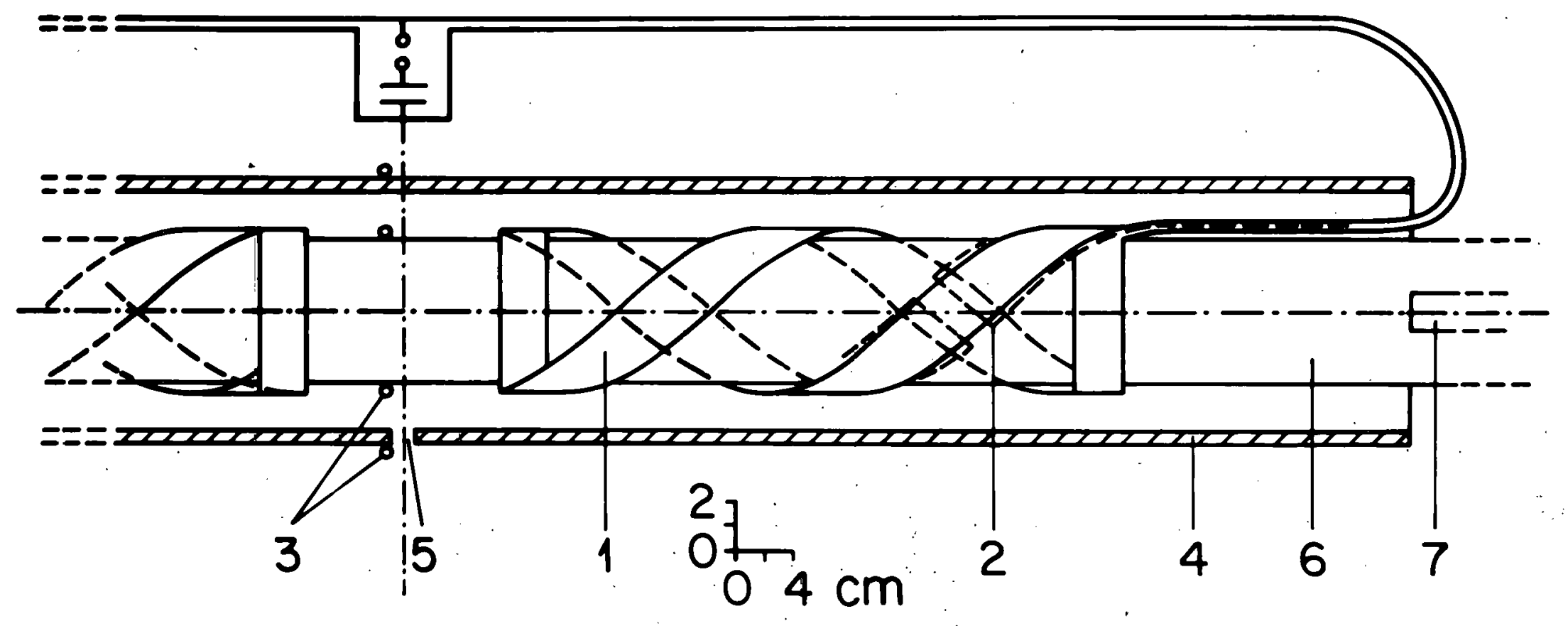




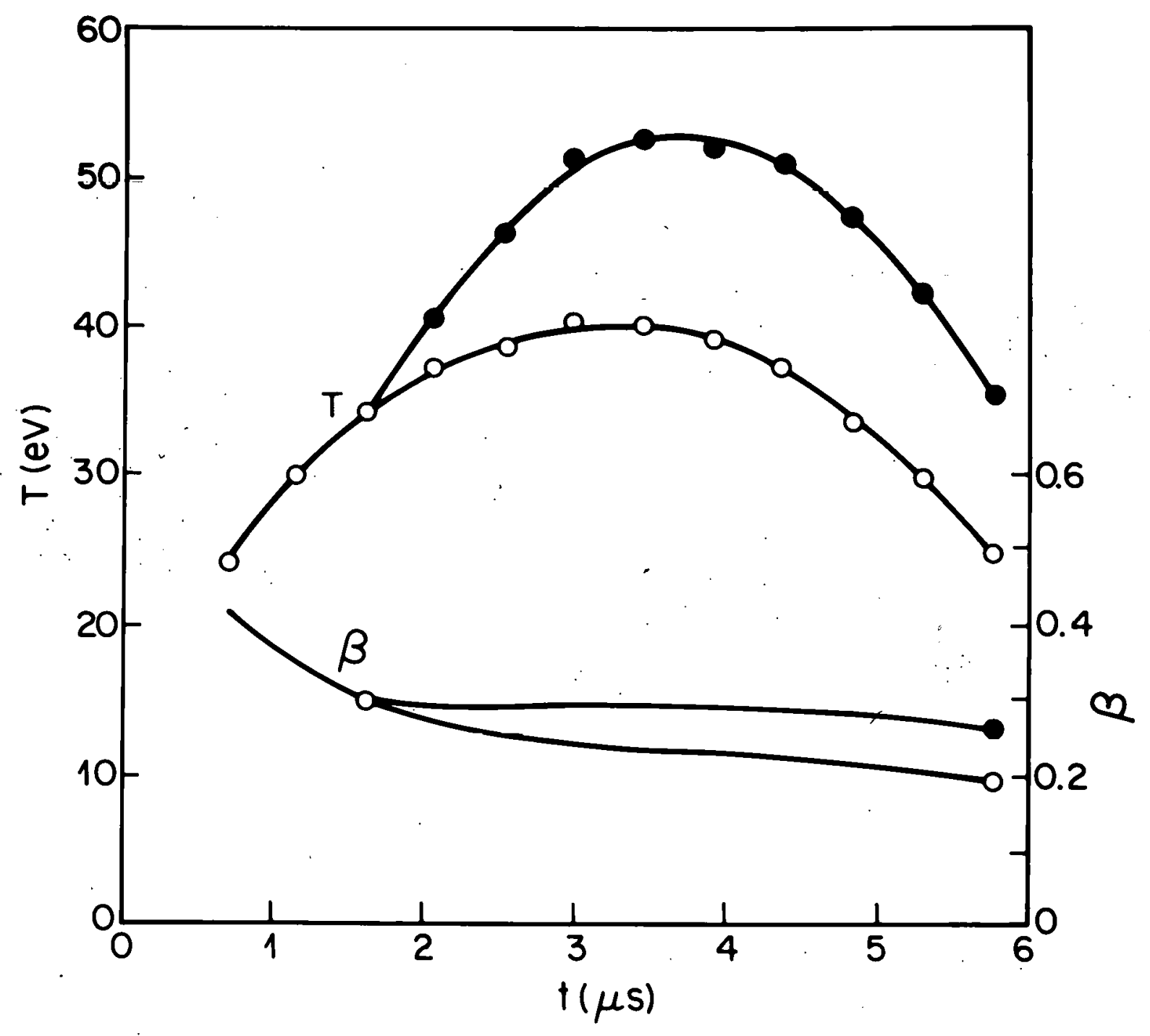




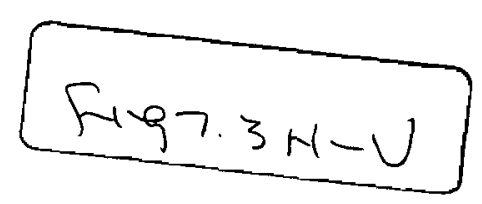




\section{PROTO - CLEO}

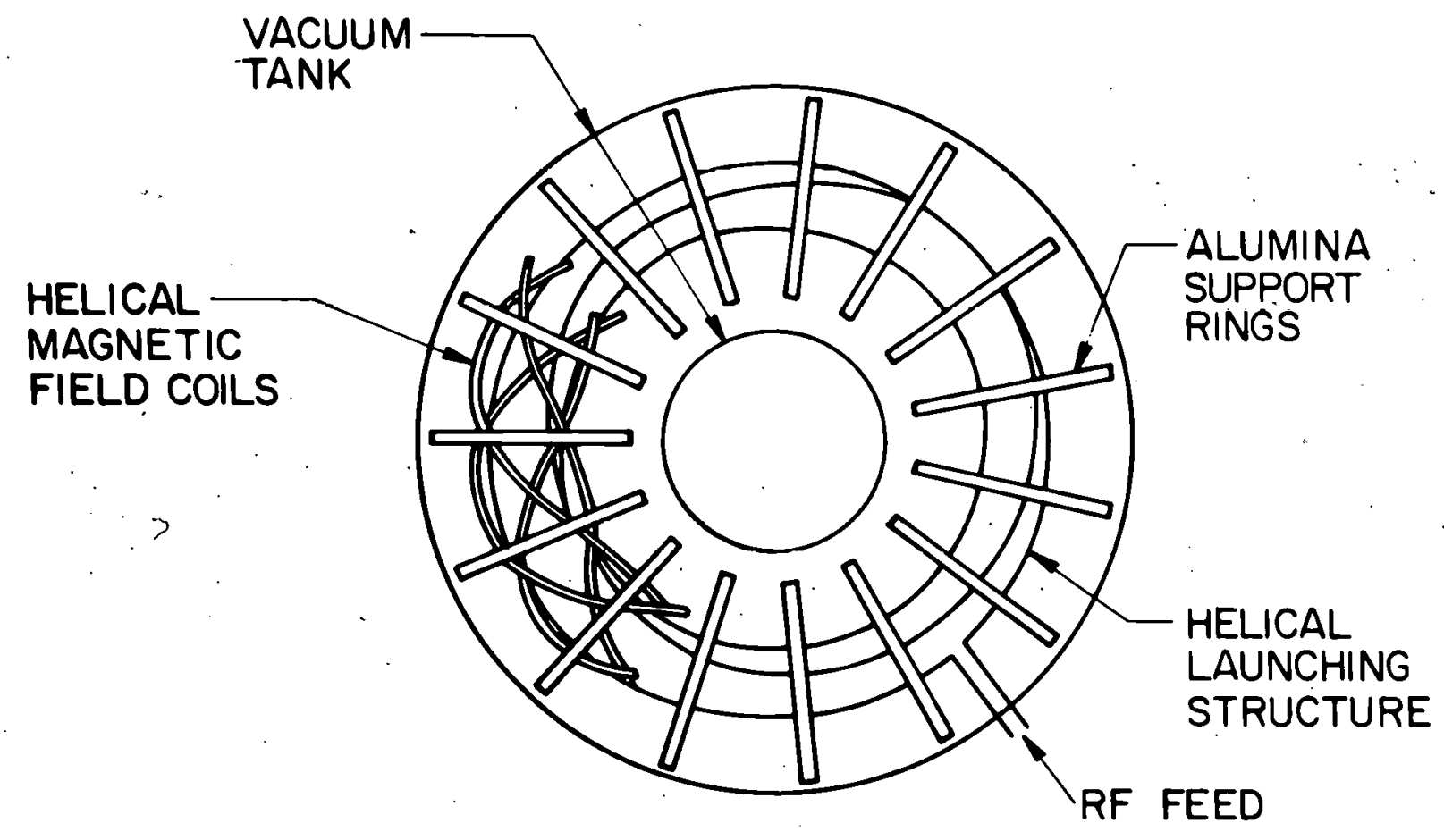





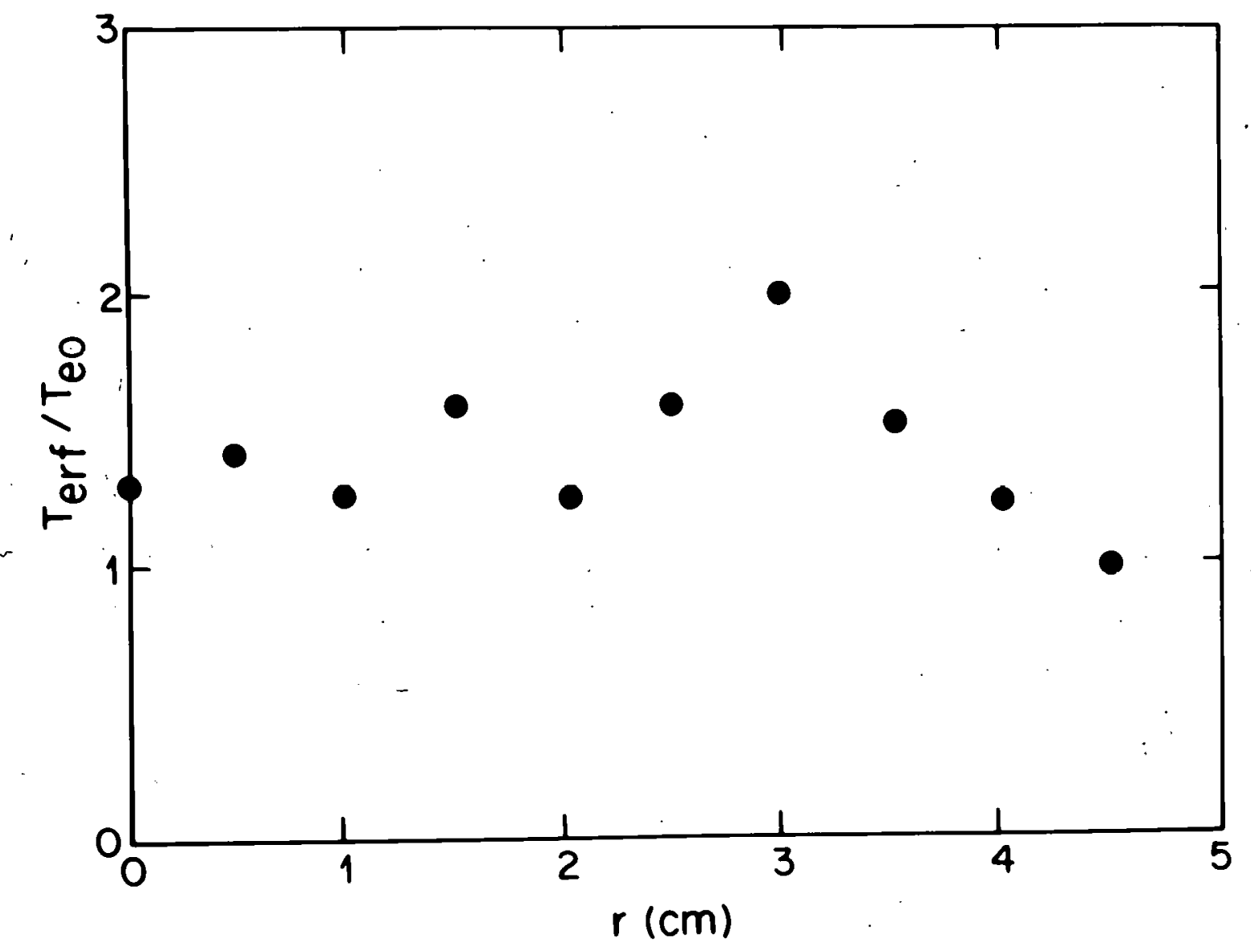


ŔO-2 STELLARATOR

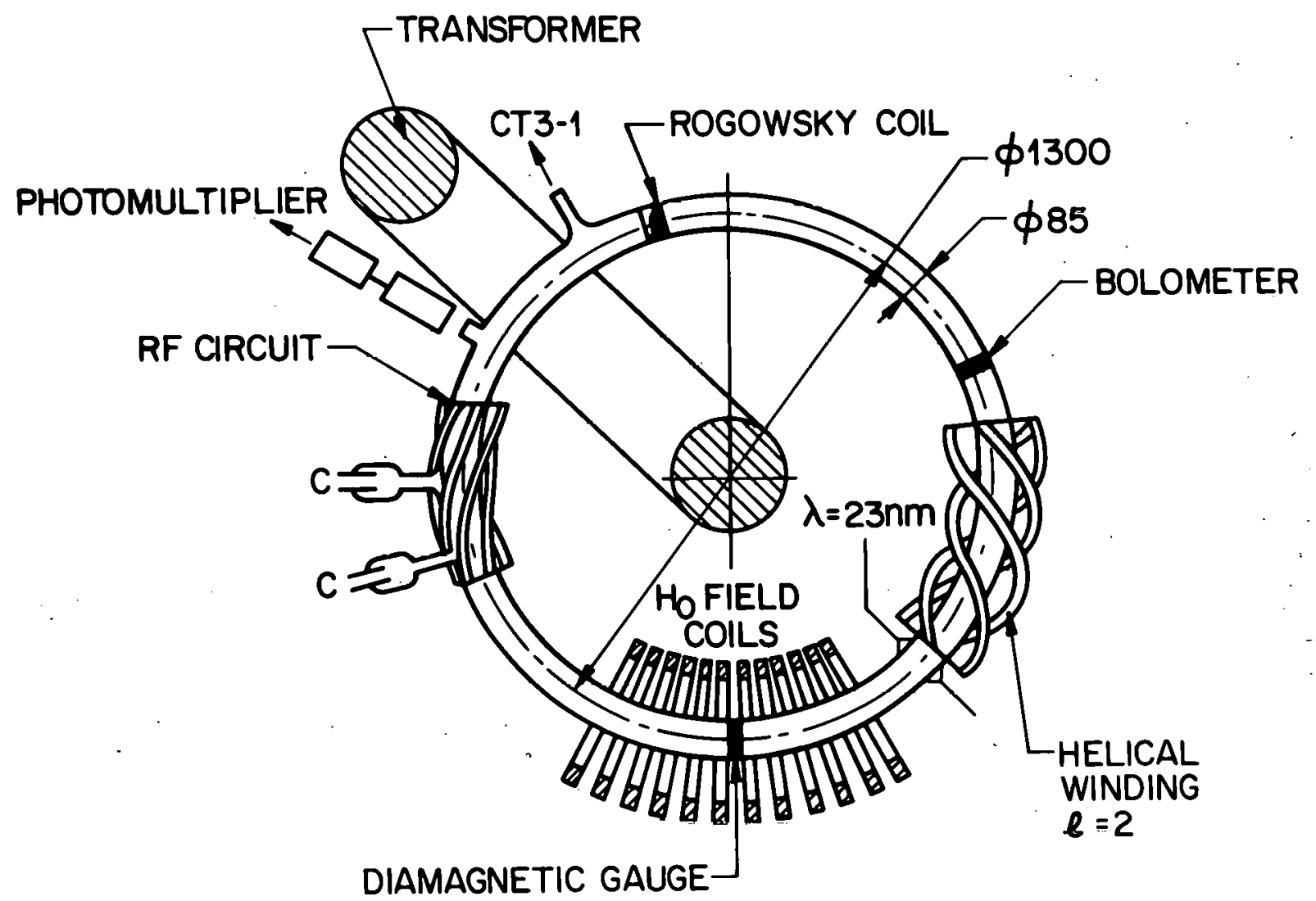




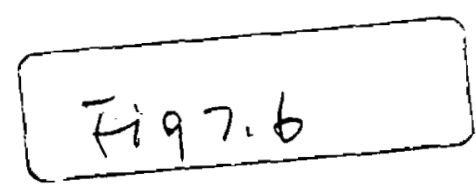




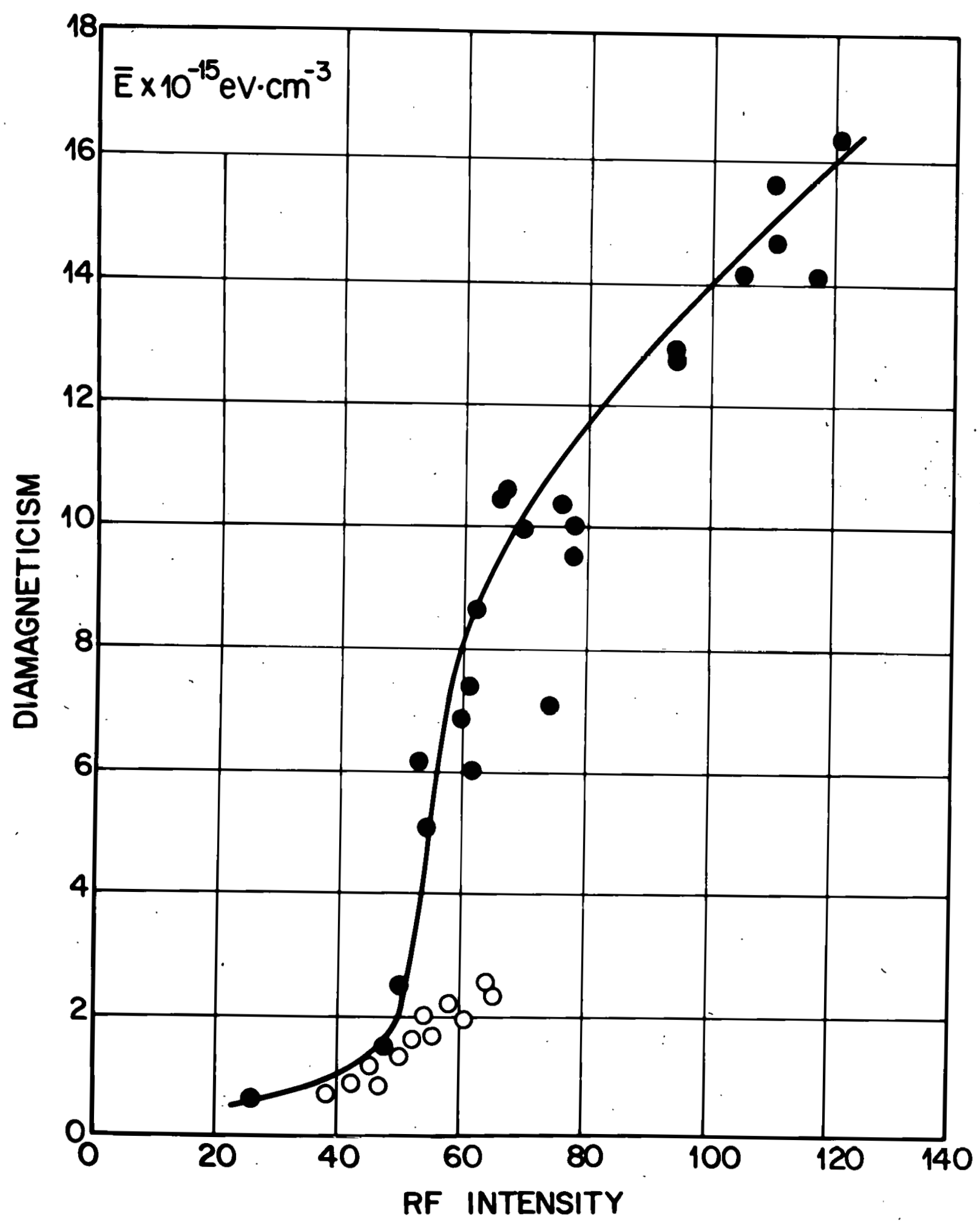


$\operatorname{Sig} 7.7 \mathrm{H}-\mathrm{U}$ 


\section{HELIOTRON-D}

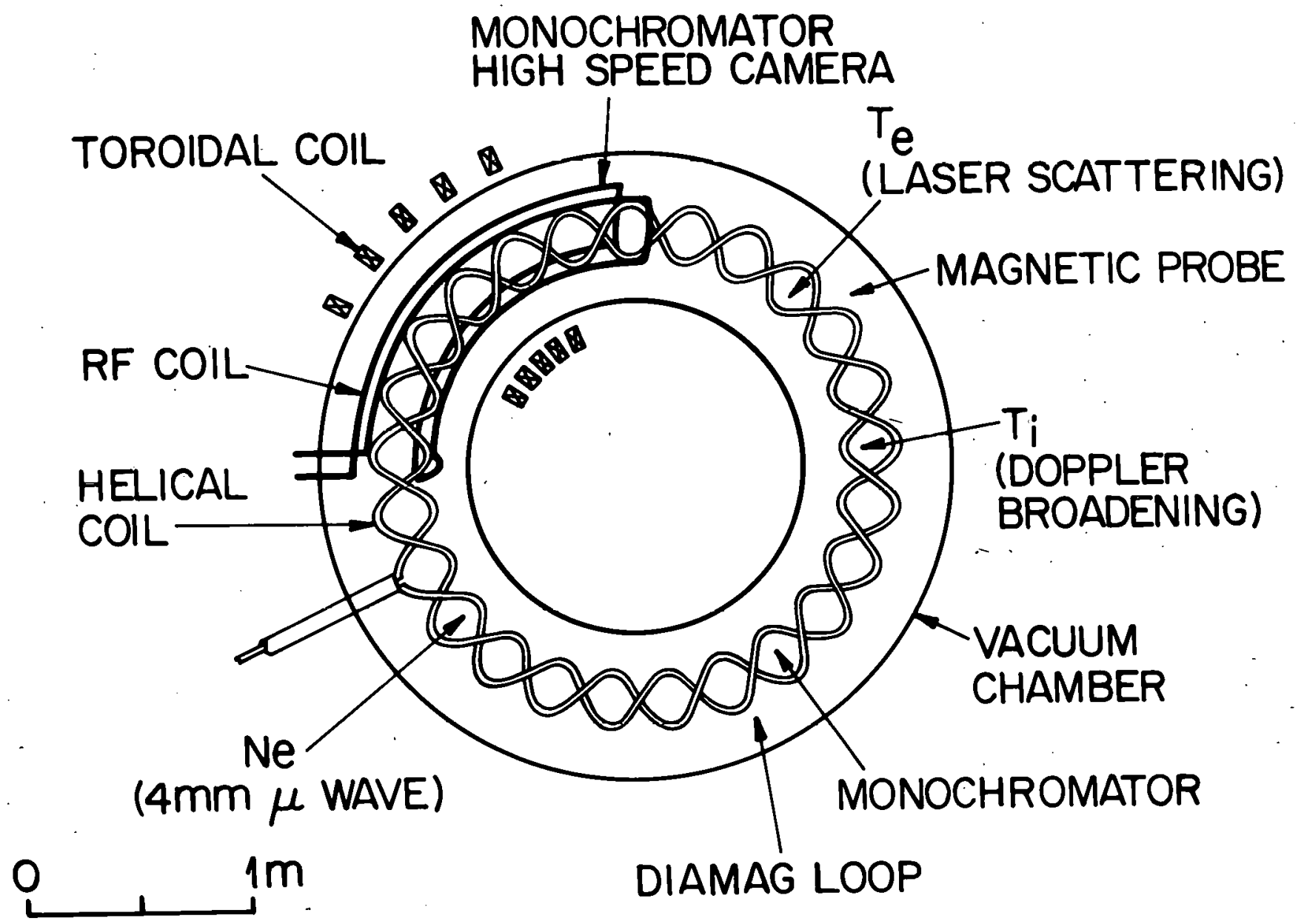


Fog $7.8 \mathrm{H}-\mathrm{U}$ 


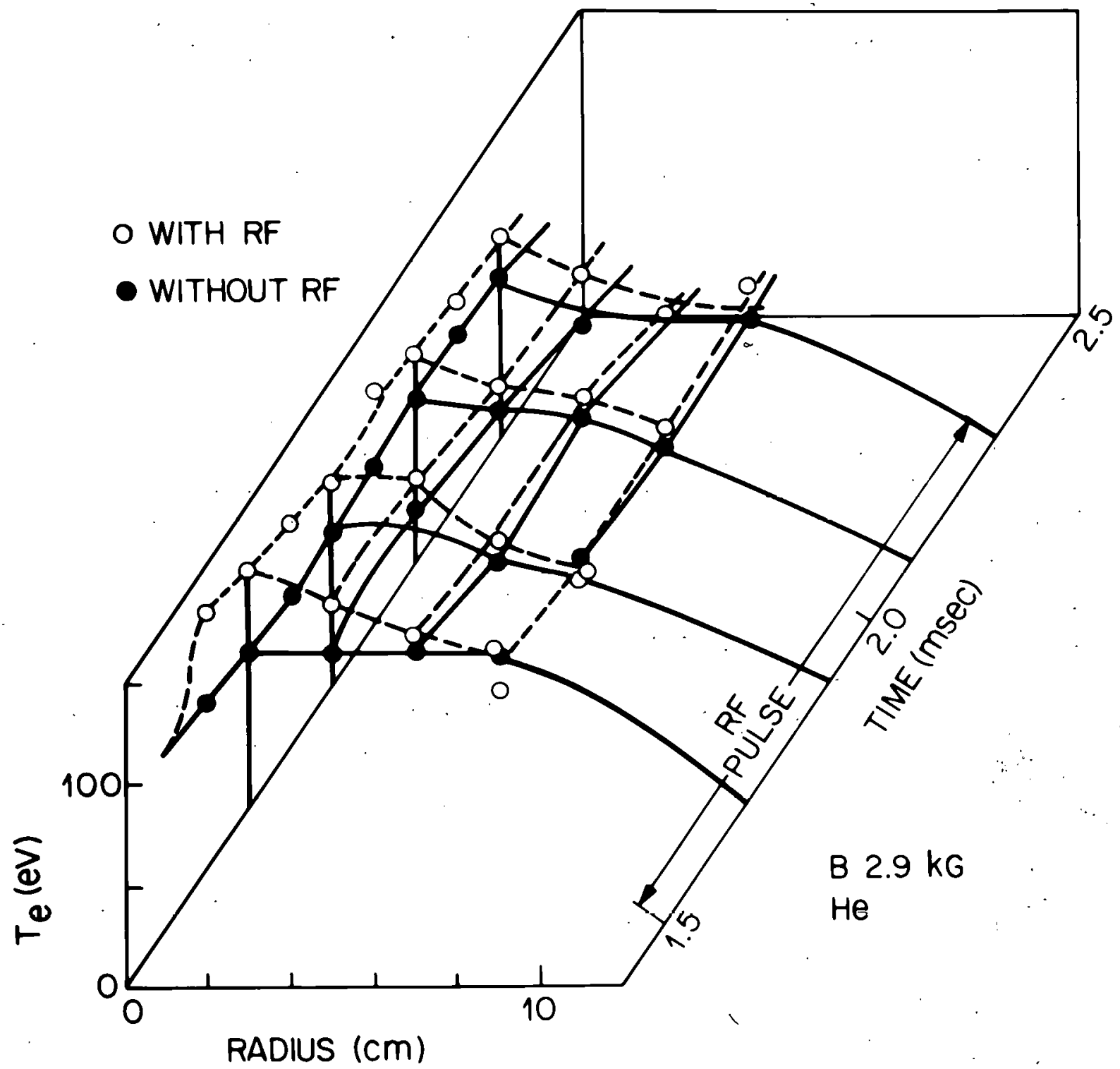


EEg 7. R. H-V 


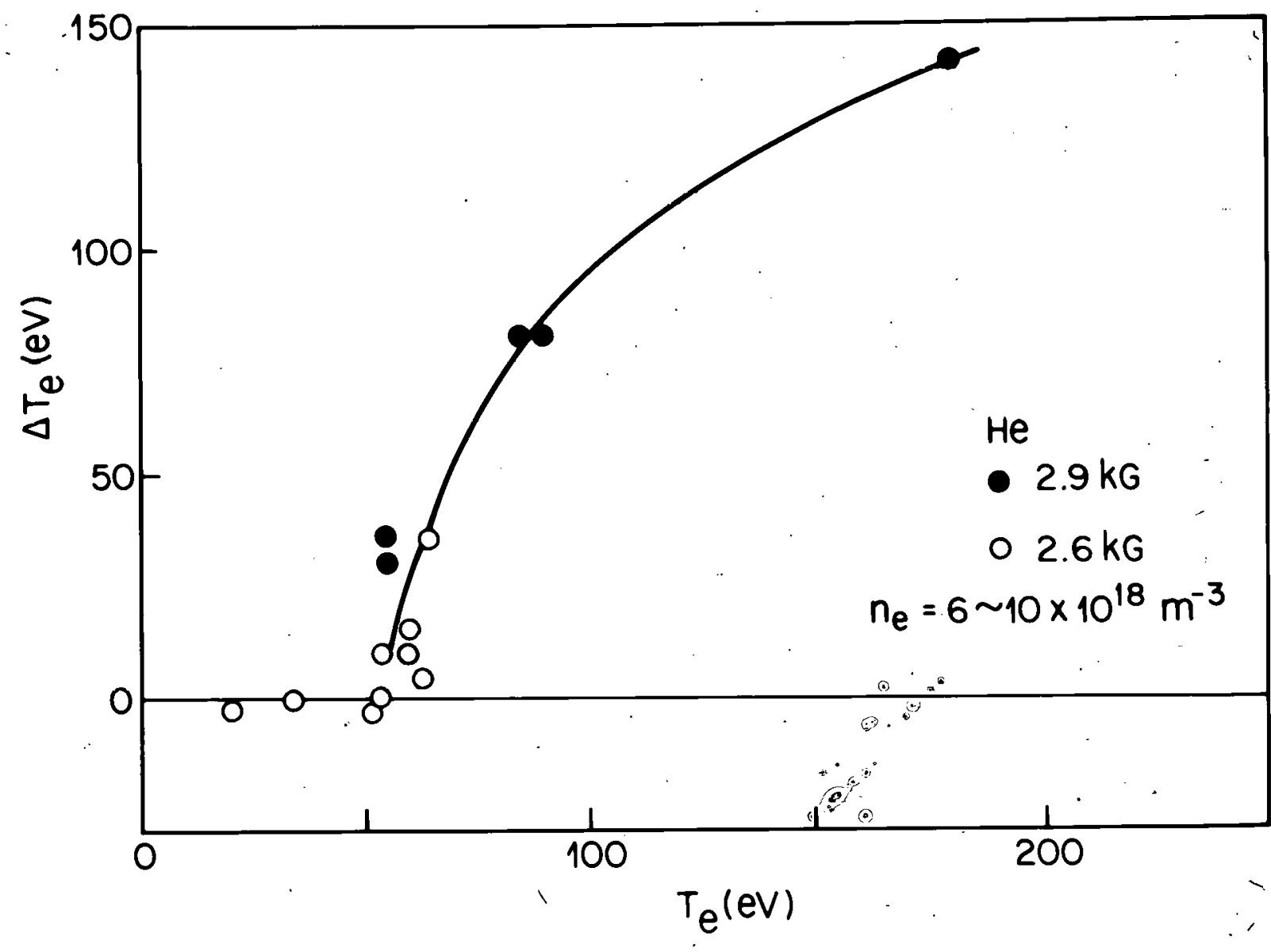


Fif? 